Susanne Voigt-Zimmermann (Hg.)

\title{
Miteinander sprechen - verantwortlich, kompetent, reflektiert
}


Susanne Voigt-Zimmermann (Hg.)

Miteinander sprechen - verantwortlich, kompetent, reflektiert 
Schriften zur Sprechwissenschaft und Phonetik, herausgegeben von Ines Bose, Kati Hannken-Illjes,

Ursula Hirschfeld, Baldur Neuber und

Susanne Voigt-Zimmermann

Band 24 
Susanne Voigt-Zimmermann

\section{Miteinander sprechen - verantwortlich, kompetent, reflektiert}




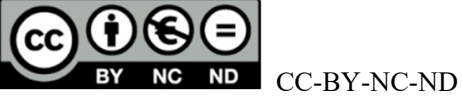

Der vorliegende Band wurde von Susanne Voigt-Zimmermann betreut.

ISBN 978-3-7329-9247-8

ISSN 2364-4494

DOI $10.26530 / / 20.500 .12657 / 49674$

(C) Frank \& Timme GmbH Verlag für wissenschaftliche Literatur Berlin 2021. Alle Rechte vorbehalten.

Das Werk einschließlich aller Teile ist urheberrechtlich geschützt. Jede Verwertung außerhalb der engen Grenzen des Urheberrechtsgesetzes ist ohne Zustimmung des Verlags unzulässig und strafbar. Das gilt insbesondere für Vervielfältigungen, Übersetzungen, Mikroverfilmungen und die Einspeicherung und Verarbeitung in elektronischen Systemen.

Herstellung durch Frank \& Timme GmbH, Wittelsbacherstraße 27a, 10707 Berlin.

Printed in Germany.

Gedruckt auf säurefreiem, alterungsbeständigem Papier.

www.frank-timme.de 


\section{Inhaltsverzeichnis}

Susanne Voigt-Zimmermann

Vorwort

Susanne Voigt-Zimmermann

„Miteinander sprechen - verantwortlich, kompetent, reflektiert“ -

Kleine Fächer-Wochen Sprechwissenschaft in Halle (Saale) 11

Kati Hannken-Illues

Sichtbar(er) werden: Die Kleine Fächer-Wochen

in Marburg und die Sprechwissenschaft

Kerstin Schuck

Ein kleines, aber kräftiges Gewächs:

Die schöne Blume „scientia orationis“ aus Jena

Susanne Voigt-Zimmermann/Stephanie Kurtenbach /

Michaela Kupietz

Sprechwissenschaftliche Therapie- und Beratungsangebote

an der Universität Halle (Saale):

Von den Anfängen bis in die Gegenwart.

Stephanie Kurtenbach / Steffi Schwarzack /

Dirk Meyer und Susanne Voigt-Zimmermann

Sprechwissenschaftliche Beratung - eine Haltungsfrage?

Matthias Winter

„Comisch“ - Coaching mit Schauspielern als konsequent

klientenzentrierte und lösungsfokussierte Methode zur

partnerorientierten Gesprächsrhetorik 
FRIDERIKE LANGE

Feedback als Haltung in der Lehrer*innenbildung im Spannungsfeld zwischen Beraten und Bewerten.

Ines Bose/Stephanie Kurtenbach /

Franziska Kreutzer und Kerstin Kreße

Mit Kindern im Gespräch. Sprechwissenschaftliche

Kita-Forschung mit Praxiskooperationen.

BALdur NeubER

Miteinander reden im digitalen Kontext

Judith Pietschmann / Alexandra Ebel

Visionen und Grenzen der digitalen Kommunikationswelt 4.0

aus sprechwissenschaftlicher Perspektive

Susanne Vollberg

Von der Suche nach dem Glück oder „Das ideale Ich“.

Selbstoptimierung im Netz 153

Alexandra Ebel/Rudolf Hientz/

Ursula Hirschfeld / Robert Skoczek

Zur Transkription von Standardsprache in

Aussprachedatenbanken: Konzepte, Probleme, Lösungen

Uwe Hollmach

Ausspracheideale heute

Julia Kiesler/Anna Wessel

Probenforschung im (sprech)künstlerischen Bereich 207

Martina Haase/Thomas Bauer-Friedrich

Die Sprechbuehne und das Kunstmuseum

Moritzburg Halle (Saale) - Geschichte einer Kooperation 


\section{Vorwort}

Im Wintersemester 2019/2020 fanden an den deutschen Hochschulstandorten der Sprechwissenschaft in Halle (Saale), Marburg und Jena die „Kleine Fächer-Woche/n Sprechwissenschaft" statt. Dabei handelte es sich um eine vom Bundesministerium für Bildung und Forschung (BMBF) ideell und finanziell geförderte Initiative der Hochschulrektorenkonferenz zur Stärkung sogenannter Kleiner Fächer. (https://www.bmbf.de/de/kleine-faecher-grossepotenziale-3041.html; https://www.hrk.de/themen/hochschulsystem/kleinefaecher/kleine-faecher-wochen-an-deutschen-hochschulen/)

„Kleine Fächer - große Potenziale“, unter diesem Motto verstehen das BMBF und die Hochschulrektorenkonferenz die Kleinen Fächer als wertvolle und traditionsreiche Elemente der vielfältigen deutschen Hochschullandschaft. Aufgrund ihrer wissenschaftlich und gesellschaftlich hohen Relevanz und ihres Reichtums an Expertise und Methoden stellen Kleine Fächer interessante Partner im interdisziplinären Austausch dar und tragen maßgeblich zur Profilbildung der Universitäten bei. Und sie erhalten durch ihr historisches Gedächtnis das kulturelle Erbe der Menschen. (https://www.hrk.de/fileadmin/redaktion/ hrk/02-Dokumente/Kleine_Faecher_grosse_Potenziale.pdf) Das Fach Sprechwissenschaft zählt mittlerweile zu den größeren der sog. Kleinen Fächer.

Jeder sprechwissenschaftliche Standort an den drei Universitäten trat mit einer eigenen Profilsetzung entweder im Projektverbund oder als Einzelprojekt an:

- die Abteilung Sprechwissenschaft und Phonetik der Martin-Luther-Universität Halle-Wittenberg mit dem Einzelprojekt „miteinander sprechen - verantwortlich, kompetent, reflektiert",

- die Arbeitsgruppe Sprechwissenschaft der Philipps-Universität Marburg im Verbundprojekt „Die weite Welt vor Ort - Der Beitrag der 
Kleinen Fächer zur Internationalisierung der Philipps Universität Marburg",

- die Professur für Sprechwissenschaft der Friedrich-Schiller-Universität Jena unter dem Motto „Alles Orchideen? Kleine Fächer-Wochen der Philosophischen Fakultät“.

Im vorliegenden Band sind nun ausgewählte Fachbeiträge zu den Veranstaltungen an der Martin-Luther-Universität Halle-Wittenberg und weiterführende Artikel sowie Berichte über die Projekte der Kleine Fächer-Wochen Sprechwissenschaft aller drei Standorte zusammengefasst. Die Beiträge und Berichte spiegeln dabei das fachliche Selbstverständnis der Sprechwissenschaft, ihre Interdisziplinarität, Internationalität und Zukunftsfähigkeit wider. Sie zeigen, wie sich die Sprechwissenschaft in ihrer über einhundertjährigen Geschichte fachlich entfaltet und weiterentwickelt hat, dass neue Forschungsthemen und praktischen Anwendungsbereiche erschlossen werden konnten und zahlreiche Forschungskooperationen im universitären, interdisziplinären und internationalen Kontext entstanden sind. In Forschung, Lehre und Praxis befasst sich die Sprechwissenschaft mit der mündlichen Kommunikation, ihren Problemen und Optimierungsmöglichkeiten in den unterschiedlichsten Bereichen, zum Beispiel auf der Bühne, in der Sprechberatung und Sprachtherapie, im Kindergarten und in der Schule, in digitalen Medien und in Aussprachedatenbanken. Die Stärken der Sprechwissenschaft liegen dabei in ihrer lebenswirklichen Verankerung und in ihrem Pragmatismus, ihrer Agilität und Vitalität. Sie wurde immer und wird zunehmend gebraucht, denn sie gibt durch ihre fachspezifische Betrachtung der komplexen mündlichen Kommunikation neue und andere Antworten auf unter Umständen alte oder scheinbar geklärte Fragen und unterbreitet Lösungen, wo manchmal kein Vorankommen mehr möglich schien.

Der vorliegende Band erscheint in der Reihe „Schriften zur Sprechwissenschaft und Phonetik" als open access Publikation. Mit einer modernen Wissenschaftskommunikation setzt er die Zielstellung der „Kleine-Fächer-Wochen“ um, nämlich eine bessere Sichtbarkeit der Sprechwissenschaft.

Da mögliche Konventionen zum Gebrauch der Gendermarkierung derzeit gesellschaftlich noch ausgehandelt werden, sind diesbezüglich in diesem hier 
vorliegenden Band unterschiedliche Formen zu sehen, denn das Deutsche bietet noch eine Fülle an Möglichkeiten, geschlechtergerecht zu formulieren.

Dem Sprechen als individueller Befähigung einerseits und dem Miteinandersprechen als Basis gesellschaftlicher Partizipation andererseits widmet sich das gesamte sprechwissenschaftliche Interesse. Der Erforschung aller Aspekte und Bedingungen der Sprechkommunikation und deren Didaktisierung im Sinne einer evidenzbasierten Sprechwissenschaft gilt unser fachliches Streben. 



\section{„Miteinander sprechen - verantwortlich, kompetent, reflektiert" - Kleine Fächer-Wochen Sprechwissenschaft in Halle (Saale)}

Zusammenfassung: Mit dem Motto „miteinander sprechen - verantwortlich, kompetent, reflektiert“ präsentierten sich bei den „Kleine Fächer-Wochen Sprechwissenschaft" in Halle alle ihre Teilbereiche in den Themenschwerpunkten „Gesundheit und Beratung“, „Politik und Öffentlichkeit“, „Medien und Digitalisierung“, „Kunst und Bühne“ sowie „Bildung und Wissenschaft“. Mit mehr als 20 Veranstaltungen hat die Sprechwissenschaft ihren Kooperationspartner*innen in Lehre, Forschung, Wissenschaft, Politik, Wirtschaft, Kultur sowie der allgemeinen Öffentlichkeit das Potenzial und die gesellschaftliche Relevanz des Faches für die Gesellschaft verdeutlichen können.

Stichpunkte: Sprechwissenschaft, mündliche Kommunikation, Potenzial, Relevanz, Kooperation

Abstract: Under the slogan "Talking with each other - responsibly, competently, reflectively" all subareas of speech science were presented at the "Small-Disciplines-Weeks Speech Science" at the University of Halle. The main topics were Health and Counseling, Politics and General Public, Media and Digitalization, Art and Stage as well as Education and Science. With more than 20 events, Speech Science presented on the potential and social relevance of the discipline for both the general public and for its cooperation partners in teaching, research, science, politics, business and culture.

Keywords: speech science, oral communication, potential, relevance, cooperation 
Die Abteilung Sprechwissenschaft und Phonetik der Martin-Luther-Universität Halle-Wittenberg (MLU) beteiligte sich erfolgreich an der Ausschreibung der Hochschulrektorenkonferenz (HRK) zur Durchführung von Kleine Fächer-Wochen (KFW) an deutschen Hochschulen und erhielt den Zuschlag für ein Einzelprojekt. Unter dem Motto „miteinander sprechen - verantwortlich, kompetent, reflektiert" präsentierten sich dann im gesamten Wintersemester 2019/2020 alle Bereiche der halleschen Sprechwissenschaft unter den Themenschwerpunkten „Gesundheit und Beratung“" „Politik und Öffentlichkeit“, „Medien und Digitalisierung“, „Kunst und Bühne“ sowie „Bildung und Wissenschaft" (Internetquelle 1).

Seit mehr als 100 Jahren befasst sich die Sprechwissenschaft an der MLU sowohl theoretisch als auch praktisch mit den Aspekten der mündlichen, d.h. der sprechsprachlichen Kommunikation von und zwischen Menschen. Zu ihren in Halle vertretenen Teilgebieten zählen die Phonetik, Rhetorik, Sprechkunst, Klinische Sprechwissenschaft und Sprechbildung. Damals wie heute strebt die hallesche Sprechwissenschaft nach einer wissenschaftlich fundierten, auf intensiver Eigenerfahrung fußenden und praxisrelevanten Ausbildung der Studierenden. Sie betreibt eine intensive Förderung des wissenschaftlichen Nachwuchses und eine enge Kooperation von Forschung, Wirtschaft, Kultur und Gesellschaft. Die Sprechwissenschaft stellt sich dabei - wie andere Wissenschaften auch - den jeweils aktuellen Herausforderungen und Fragen der Gesellschaft, die ihr wissenschaftliches Selbstverständnis ebenso berühren wie ihre Paradigmen, die Methodik, Didaktik und den Transfer. Denn die gesprochene Sprache, das Miteinandersprechen von Menschen unterliegt neben allgemein gesellschaftlichen auch fortwährenden sozialen, politischen, technologischen und kulturellen Einflüssen, vielleicht so stark wie keine andere Daseinsform der Menschen. Die Sprechwissenschaft arbeitet überwiegend inter- und transdisziplinär, sie greift technologische Entwicklungen auf und intergiert diese in Forschung, Lehre und Praxis. Davon und von ihrer gesellschaftlichen Relevanz sowie ihrem Potenzial für den Einzelnen und die gesamte Gesellschaft gaben die verschiedenen Veranstaltungen und Events der KFW Sprechwissenschaft beredt Auskunft. 


\section{Ziele der „Kleine Fächer-Wochen Sprechwissenschaft“}

Eines der Ziele der KFW Sprechwissenschaft war es, alten und neuen Kooperationspartner*innen in Lehre, Forschung, Praxis, Kultur, Politik und Gesellschaft das Potenzial der Sprechwissenschaft aufzuzeigen und ihre Stärken sichtbar und erfahrbar zu machen, ihre Leistungen für Wissenschaft und Alltag zu verdeutlichen und ihre Zukunftsfähigkeit durch eine stärkere hochschulinterne und -externe Kooperation zu festigen. Ein weiteres, sehr wichtiges Ziel war die Akquise von potentiellen Studienbewerber*innen. Aus diesem Grund wurde im Rahmen der KFW ein Imagefilm der Sprechwissenschaft an der MLU erstellt, der das Fach, die Studieninhalte und -bedingungen sowie die beruflichen Möglichkeiten der Absolvent ${ }^{*}$ innen vorstellt (Internetquelle 2). Durch die KFW sollten zudem das Bewusstsein für die Bedeutung und die Transferstärke des bundesweit seltenen Fachs geschärft werden. Ein weiteres Ziel war es dabei öffentlich darüber ins Gespräch zu kommen, was die Sprechwissenschaft, die Zivilgesellschaft und jede/r Einzelne - unter Umständen mit deren Hilfe - dazu beitragen können, das Führen und die Teilhabe an gesellschaftlichen Diskursen in Gang zu setzen und zu unterstützen. Die Notwendigkeit des Sprechens über das Miteinandersprechen wurde und wird angesichts der zunehmend kontroversen, hasserfüllten bis unmöglich gewordenen gesellschaftlichen und sozialen Debatten noch verstärkt. Denn immer klarer kristallisiert sich heraus: „Wie wir heute miteinander sprechen, werden wir morgen miteinander leben“.

\section{Unterstützung der „Kleine Fächer-Wochen Sprechwissenschaft"}

Die Martin-Luther-Universität Halle-Wittenberg hat die „Kleine Fächer-Wochen Sprechwissenschaft“ (KFW SW) in vielfältiger Weise gefördert, denn kleine Fächer sind zwar nicht groß, aber unverzichtbar. Die Unterstützung erfolgte vor allem durch das Rektorat und insbesondere im Rahmen der Antragstellung durch den Rektor Herrn Prof. Christian Tietje sowie die Pressereferentin Frau Dr. Bank-Zillmann. Die universitäre Unterstützung fand außerdem durch die 
Prorektorin für Personalentwicklung und Struktur, Frau Prof. Johanna Mierendorff, und den Prorektor für Studium und Lehre, Herrn Prof. Wolf Zimmermann, statt, die beide durch ihre Vorträge und Grußwort maßgeblich zum Erfolg der Veranstaltungen beigetragen haben.

Dass auch die Landesregierung Sachsen-Anhalts hinter den Kleinen Fächern ihrer Hochschulen steht, haben die engagierten Grußworte der Ministerin für Arbeit, Soziales und Integration des Landes Sachsen-Anhalt, Frau Petra Grimm-Benne, anlässlich der Ausstellungseröffnung „Was Sprachtherapie kann“ am 08. November 2019 eindrücklich unter Beweis gestellt.

Eine weitere, sehr wichtige Unterstützerin der KFW SW an der MLU war die Nationale Akademie der Wissenschaften Leopoldina. Frau Dr. Kathrin Happe, Referentin und Stellvertretende Leiterin der Abteilung Wissenschaft - Politik - Gesellschaft der Leopoldina, sowie Frau Dr. Stefanie Westermann, ebenfalls Referentin der genannten Abteilung, waren von der ersten Idee bis zur erfolgreichen inhaltlichen und personellen Ausgestaltung sowie Kommunikation über die gemeinsame Veranstaltung „Im Gespräch bleiben - Wie wir heute über konflikthafte Themen in Politik und Gesellschaft sprechen" maßgeblich beteiligt.

Berufsständische Unterstützung ideeller und finanzieller Art erfolgte durch den Mitteldeutschen Verband für Sprechwissenschaft und Sprecherziehung (MDVS), die Deutsche Gesellschaft für Sprechwissenschaft und Sprecherziehung (DGSS), den Deutsche Bundesverband für akademische Sprachtherapie und Logopädie (dbs) sowie den Deutschen Bundesverband für Klinischer Sprechwissenschaftler (DBKS).

\section{Veranstaltungen der „Kleine Fächer-Wochen Sprechwissenschaft"}

\section{Ausstellung „Was Sprachtherapie kann“}

Den Beginn der KFW SW an der MLU bildete am 08. November 2019 die Ausstellungseröffnung: „Was Sprachtherapie kann“ (Konzeption: Beate Stoye) im Ratshof der Rathauses der Stadt Halle. Die Ausstellung präsentierte von Herbst 2019 bis Frühjahr 2020 in beeindruckenden Porträtbildern von Patient ${ }^{*}$ innen, 
was Sprachtherapie für die Betroffenen im Ringen um soziale, gesellschaftliche und berufliche Teilhabe sowie für die Gesellschaft zu leisten vermag. Zur Vernissage kamen (Klinische) Sprechwissenschaftler*innen und andere akademische Sprachtherapeut ${ }^{\star}$ innen mit Patient ${ }^{\star}$ innen und Vertreter ${ }^{\star}$ innen aus dem Gesundheitswesen und der Gesundheitspolitik ins Gespräch.

Eröffnet wurde die Veranstaltung durch die Sozialministerin des Landes Sachsen-Anhalt Petra Grimm-Benne, durch die Beigeordnete der Stadt Halle, Katharina Brederlow, die Prorektorin für Personalentwicklung und Struktur Prof. Dr. Johanna Mierendorff sowie die Sprecherin der Abteilung Sprechwissenschaft und Phonetik an der MLU Prof. Dr. Susanne Voigt-Zimmermann (Abb. 1). Die Ministerin machte darauf aufmerksam, was Sprechwissenschaft bedeutet und wie wichtig die Arbeit akademischer Sprachtherapeut*innen nach ihrer universitären Ausbildung ist. „Es müsste noch viel mehr von ihnen geben", stellte die Ministerin mit Blick auf die Ziele der Ausstellung fest (Internetquelle 3). Diesem Appell schlossen sich der Vorsitzende des Bundesverbandes für akademische Sprachtherapie und Logopädie Prof. Dr. Michael Wahl sowie die Urheberin der Ausstellung und Vertreterin des dbs in Sachsen-Anhalt von Beate Stoye in ihren Grußworten an (Internetquelle 4).

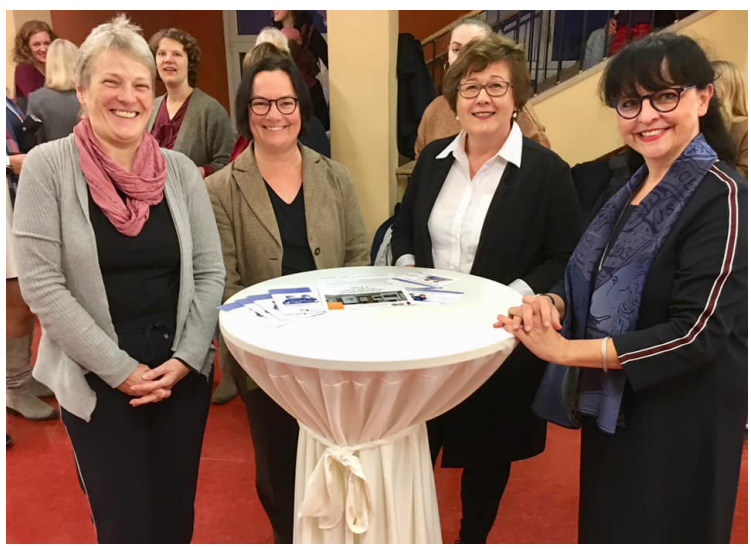

Abb. 1: Ausstellungseröffnung „Was Sprachtherapie kann“ Katharina Brederlow, Beigeordnete für Bildung und Soziales der Stadt Halle, Prof. Johanna Mierendorff, Prorektorin für Personalentwicklung und Struktur der MLU, Petra Grimm-Benne, Ministerin für Arbeit, Soziales und Integration Sachsen-Anhalts, und Prof. Susanne Voigt-Zimmermann, Sprecherin der Abteilung Sprechwissenschaft und Phonetik der MLU (von links nach rechts, Foto: Markus Scholz) 


\section{Eröffnung der „Sprechwissenschaftlichen Beratungsstelle“ (BESS) mit der Tagung „Beratung in Forschung, Lehre und Praxis“}

Am 11. November 2019 fand auf dem Steintor-Campus der MLU die feierliche Eröffnung der „Sprechwissenschaftlichen Beratungsstell“" (BESS) mit einer Fachtagung „Beratung in Forschung, Lehre und Praxis“ und der Präsentation eines Imagefilmes zur BESS statt (Internetquelle 5). Würdigende Grußworte der Universität überbrachte der Prorektor für Studium und Lehre, Prof. Wolf Zimmermann. Der Transfer sprechwissenschaftlicher Therapie- und Beratungsexpertise in die Uni, die Stadt und das Land hat eine lange Tradition. Davon berichtet der Beitrag „Sprechwissenschaftliche Therapie- und Beratungsangebote: Von den Anfängen bis in die Gegenwart" von Prof. Dr. Susanne Voigt-Zimmermann, Dr. Stephanie Kurtenbach und Michaela Kupietz in diesem Band. Das durch die Veranstaltung ausgelöste Interesse an dieser Einrichtung war enorm hoch und mündete beispielsweise in einem Bericht des Deutschlandradios (Internetquelle 6).

Verbunden mit der sprechwissenschaftlichen Beratung ist die immer wiederkehrende Frage der, inneren Haltung ' zum Beruf als Berater ${ }^{\star}$ in oder der Beratungssituation ganz allgemein. Dieser Frage geht das im vorliegenden Band veröffentlichte Interview „Sprechwissenschaftliche Beratung - eine Haltungsfrage?" von Dr. Stephanie Kurtenbach, Dirk Meyer, Steffi Schwarzack und Prof. Dr. Susanne Voigt-Zimmermann nach. Seine in der sprechwissenschaftlichen Beratung innovative Methode „Comisch“ - ein Coaching mit Schauspieler*in stellt Matthias Winter mit seinem Beitrag vor.

\section{Tagung „Ausspracheideale auf der Bühne in Geschichte und Gegenwart“}

Die hallesche Sprechwissenschaft, insbesondere durch Prof. Dr. Ursula Hirschfeld vertreten, arbeitet seit längerem im Forschungsprojektes „Wagner-Lesarten“ (Leitung: Kent Nagano) mit. Ihren Ausdruck fand diese Praxis- und Forschungskooperation am 15. und 16. November 2019 durch die internationale und interdisziplinäre Fachtagung „Ausspracheideale auf der Bühne in Geschichte und Gegenwart“, die zusammen mit „Freunde von Concerto Köln e. V.“ ausgerichtet wurde. Mit seinem Grußwort würdigte Kent Nagano die 
Idee einer historisch informierten und gleichsam wissenschaftlich basierten Aufführung Wagners „Ring der Nibelungen“, wozu die Textarbeit bzgl. Aussprache, Intonation, Inhalt und Kontext gehört, die die Basis der sicherlich als unikal zu bezeichnenden wissenschaftlich-künstlerischen Zusammenarbeit darstellt (Internetquelle 7).

Von der langen Forschungstradition der Sprechwissenschaft zur Orthoepie und ihren aktuellen Fragen an sich selbst und im künstlerischen Kontext berichten die beiden Beiträge in dem vorliegenden Band von Dr. Alexandra Ebel, Rudolf Hientz, Prof. Dr. Ursula Hirschfeld und PD Dr. habil. Robert Skoczek „Zur Transkription von Standardsprache in Aussprachedatenbanken: Konzepte, Probleme, Lösungen“ und von Prof. Dr. Uwe Hollmach „Ausspracheideale heute“.

Der Tagung vorangestellt war ein von Dr. Julia Kiesler (Bern) moderierter Workshop für Nachwuchswissenschaftler*innen, die ihre Promotions- bzw. Forschungsprojekte im Themenkreis „Sprechen und Singen auf der Bühne“ zur Diskussion stellten. Anna Wessel und Julia Kiesler geben in dem Beitrag „Probenforschung im (sprech)künstlerischen Bereich" gemeinsam einen Einblick in eines der insgesamt außerordentlich interessanten Themen des Workshops.

Die künstlerisch-thematische Einstimmung übernahmen am Abend Rika Eichner (Köln) und Volker Krafft (Hamburg) mit einem beindruckenden Vortragskonzert zum Thema „Das Sänger/-innen-Ideal von Julius Hey“.

Ulrike Wilckens (München) moderierte die abschließende Podiumsdiskussion zum Thema „Singen und Sprechen auf der Bühne“, auf der die Mezzosopranistin und berühmte Wagner-Interpretin Lioba Braun (München/Köln) sowie die Sprechwissenschaftler*innen Prof. Dr. Uwe Hollmach (München/ Halle) und Dr. Julia Kiesler aus ihren jeweils fachlichen Perspektiven berichteten.

Im Rahmen dieser Tagung fand nicht nur ein ganz klar auf die Zukunft gerichteter fachlicher Austausch statt, sondern es bildeten sich auch eindrücklich die enge Verbindung von Wissenschaft und Sprechkunst seit dem 19. Jahrhundert sowie die fruchtbare Zusammenarbeit zwischen Sprech- und Musikwissenschaft ab. Die Sprechwissenschaft gehört an der MLU deshalb völlig zurecht zum 2017 neu gegründeten Institut für Musik, Medien- und Sprechwissenschaften (IMMS). 
Ringvorlesung „Ideale in Musik, Gesang, Medien, Sprache und Sprechen“

Begleitet wurde deshalb die KFW Sprechwissenschaft durch eine gemeinsame und das gesamte Wintersemester 2019/21 stattfindende öffentliche Ringvorlesung des IMMS zum Thema „Ideale in Musik, Gesang, Medien, Sprache und Sprechen“. Mit dieser Ringvorlesung wurden die Grundlagen, die Entstehung und Entwicklung sowie die theoretischen und methodisch-didaktischen Implikationen thematisiert, diskutiert und auch hinterfragt, die Idealbildungen für Lehre, Forschung und Praxis mit sich bringen. Der „Goldstandard“, den das Ideal für sich als Ausgangspunkt bzw. Ziel gern reklamiert, stellt sich jedoch im „wirklichen“ Leben oftmals als nicht umsetzbar dar. In den verschiedenen Erlebniswelten von Musik, Gesang, Medien, Sprache und Sprechen wird er mitunter sogar als nicht erstrebenswert angesehen. Die Ringvorlesung ging deshalb auch folgenden Fragen nach: Wie umgehen mit Idealen und auch Idolen? Wie sie untersuchen, definieren und lehren? (Internetquelle 8). Frau Prof. Dr. Susanne Vollberg von der Medien- und Kommunikationswissenschaft thematisierte die zunehmende Suche nach dem Glück oder dem „idealen Ich“ als Prozess der Selbstoptimierung im Netz. Davon berichtet ihr Artikel in diesem Band.

\section{Podiumsdiskussion „Im Gespräch bleiben - Wie wir heute über konflikthafte Themen in Politik und Gesellschaft sprechen“}

Ein weiterer Höhepunkt war die Podiumsdiskussion „Im Gespräch bleiben - Wie wir heute über konflikthafte Themen in Politik und Gesellschaft sprechen", die in Kooperation mit der Nationalen Akademie der Wissenschaften „Leopoldina“ am 18. November 2019 im Vortragssaal der Leopoldina Halle stattfand. Unter der Moderation von Frau Anja Heyde (ZDF/MDR) diskutierten die Politiker Dr. Christoph Bergner (Ministerpräsident Sachsen-Anhalts a.D., CDU) und Sebastian Striegel (MdL, Bündnis 90/Die Grünen), der Politikdidaktiker Prof. Dr. Andreas Petrik (MLU), der Sozialpsychologe PD Dr. Oliver Decker (Universität Leipzig) sowie die Sprechwissenschaftlerin Prof. Dr. Susanne Voigt-Zimmermann (MLU) (Internetquelle 9). 
Ziel der Podiumsdiskussion war es, die Bedeutung des Miteinandersprechens für das gesellschaftliche und soziale Miteinander bewusst zu machen. Dabei ging es auch darum, Möglichkeiten der aktiven, partizipativen und gleichberechtigten Ausgestaltung von Gesprächen zu suchen. Deshalb kamen die Diskutant*innen nach der Podiumsdiskussion mit Bürger*innen ins Gespräch.

Die Medienresonanz der Diskussion war enorm. So kam beispielsweise die Zeitschrift „Die politische Meinung“ der Konrad-Adenauer-Stiftung auf die hallesche Sprechwissenschaft zu, für die Prof. Dr. Susanne Voigt-Zimmermann und Dr. Judith Pietschmann in dem Fachbeitrag zur Kunst des Miteinandersprechens aus einer sprechwissenschaftlichen Perspektive auf die Qualität des aktuellen gesellschaftlichen Diskurses schauen (Voigt-Zimmermann/Pietschmann 2020).

\section{Workshop „Chorisches Sprechen“ mit Bernd Freytag}

Am 29. und 30. November fand dann ein sprechkünstlerischer Workshop zum Thema „Chorisches Sprechen“ mit Bernd Freytag statt. Mit dem Workshop wurde vor allen Dingen den Studierenden die Möglichkeit der praktischen Arbeit eingeräumt. Herr Freytag ist Autor und einer der renommiertesten Sprechchorregisseure im deutschsprachigen Theaterraum. Er arbeitete u.a. mit Einar Schleef und Frank Moritz als Chordarsteller, Chorleiter, Schauspieler und Regieassistent. Exemplarisch für die vielen sehr positiven Rückmeldungen, soll hier die Master-Studentin Sophia Güttler zu Wort kommen: „Die Verbindung von Theorie und Praxis ist unfassbar wichtig. Ich danke der Hochschule und der Hochschulrektorenkonferenz (HRK) sehr, dass sie Projekte wie diese gefördert haben und kann nur sagen: Bitte weiter so. Das sind Sternstunden der Sprechwissenschaft in lebendigem Lernen. Es gibt kaum eine bessere Verknüpfung als selbst aktiv zu werden und als Teil der Gruppe Erfahrungen zu sammeln. Da werden Begriffe griffig-greifbar und Sinnhaftigkeit zieht ein in dieses sinnig-sinnliche Fach der Sprechkunst/ Sprechwissenschaft." (Internetquelle 10) 


\section{Internationaler Doktorandentag}

Die Sprechwissenschaft Halle ist seit vielen Jahrzehnten international stark verbunden, so etwa in Erasmus-Projekten und anderen Forschungs- und Lehrverbünden, wie dem sog. Vladimir-Admoni-Programm (Internetquelle 11). Außerdem ist sie intensiv in der Ausbildung und bei Förderung des wissenschaftlichen Nachwuchses engagiert. Beide für ein kleines Fach überlebenswichtigen und essentiellen Aktivitäten wurden während der KFW SW durch einen Internationaler Doktorandentag am 3. Dezember 2019 zusammengeführt. Zehn Promovendinnen der Sprechwissenschaft präsentierten dabei in Vorträgen und einer Poster- Präsentation ihre Forschungsprojekte und -ergebnisse von Studien und Projekten aus Russland, Japan, China und Deutschland. Neben fachlichen und forschungsmethodischen Aspekten wurden themenund zielgruppenspezifische Forschungsmöglichkeiten der Sprechwissenschaft gezeigt und das hohe Vernetzungspotenzial insbesondere auf internationaler Ebene dargestellt.

\section{Vorlese-Karawane durch die Stadt Halle (Saale)}

Die Studierenden der halleschen Sprechwissenschaft waren nicht nur an den Vorbereitungen, organisatorischen Begleitung und Nachbereitung aller Veranstaltungen der KFW SW beteiligt, sondern haben auch mit eigenen Veranstaltungen an der Umsetzung der Ziele der KFW erfolgreich mitgewirkt. Dazu gehörte die Vorlese-Karawane der Studierenden der Sprechwissenschaft am 06. Dezember 2019 durch caritative und klinische Einrichtungen der Stadt Halle. Am Nikolaustag machten die Studierenden aus verschiedenen Semestern Kindern sowie jungen und alten Erwachsenen durch das Vorlesen von Märchen, Geschichten, Gedichten und das Singen von Winter- und Weihnachtsliedern eine große Freude. Die Karawane machte beispielsweise Station im Kindergarten am Moritzburg-Ring, in der Klinik für Kinderheilkunde des St. Elisabeth-Krankenhauses Halle, in der Senioren-Tagespflege Steintor-Palais und auf dem Halleschen Weihnachtsmarkt. Mit dieser Vorleseaktion präsentierten die Studierenden der Sprechwissenschaft ihre im Studium der Leselehre, Sprechbildung, sprechkünstlerischen Interpretation und Kommunikation 
erworbenen Kompetenzen, Fähigkeiten und Fertigkeiten. Es handelte sich also um einen Transfer sprechwissenschaftlicher Expertise in die Gesellschaft im allerbesten Sinne.

\section{Führung durch die „Phonetische Sammlung“ und Jahreskalender}

Zum ,Tafelsilber' der Sprechwissenschaft gehört die berühmte „Phonetische Sammlung", die 1910 von dem halleschen Phonetiker Otto Brehmer gegründet und 1936 von Richard Wittsack in die damals noch sog. Sprechkunde integriert wurde. Er selber hatte seit 1925 ebenfalls eine eigene sprechkundliche Sammlung aufgebaut. Sie besteht aus einer großen Anzahl von Objekten und Geräten, wie Phono-, Parlo- und Kymographen von Anfang 1900 und dem dazugehörigen Schallarchiv mit u.a. Wachs-Walzen, Schellackplatten, Decelith-Folien und Magnettonbändern.

Am 19. Dezember fand gleichsam als Ausklang des Jahres 2019 eine Führung durch diese Phonetische Sammlung statt. Dipl.-Ingenieur Peter Müller gab profund und unterhaltsam interessante Einblicke in dieses nicht nur sprechwissenschaftliche, sondern auch kulturelle Erbe der Menschheit. Die öffentliche Führung unterstützte damit das historische Gedächtnis an diese einzigartige Sammlung und die Anfänge der Sprechwissenschaft.

Es wurde zudem einen Jahreskalender 2020 mit kommentierten Abbildungen präsentiert, der den Kooperationspartner*innen, den Vertreter ${ }^{\star}$ innen der Universität und der Stadt sowie allen Unterstützer*innen der KFW SW in Halle übergeben werden konnte. Hierbei handelt es sich sicherlich um eine der schönsten Formen von Wissenschaftskommunikation.

\section{Workshoptag zur Feedbackkultur in der Lehrerinnenbildung}

Die Sprechwissenschaft hat eine ihrer fachgeschichtlichen Wurzeln in der Lehrer*innenbildung. Ein starkes Interesse rief deshalb am 11. Januar 2020 der Workshoptag zur Feedbackkultur in der Lehrer*innenbildung hervor. Das Ziel dieses praxisorientierten Tages war es, verschiedene Facetten rund um das Thema Feedbackkultur in der Ausbildung und Schule zu betrachten und praktisch auszuprobieren. Es wurden gemeinschaftlich Antworten auf die Frage gesucht 
„Wie kann das Miteinander-Sprechen im Rahmen einer Feedbackkultur gestaltet werden, in der Scheitern erlaubt ist und ein achtsamer und (ko-)konstruktiver Lernprozess im Gespräch stattfinden kann?“. Es nahmen mehr als 70 Studierende, Lehrer*innen im Vorbereitungsdienst, Lehrkräfte im Schuldienst, Mentor ${ }^{\star}$ innen, Fachdidaktiker*innen, Haupt- und Fachseminarleiter*innen teil und viele, die sich für das Thema Lehrer*innenbildung interessieren. Ein eingehender Bericht zu diesem Workshoptag wurde auf den Seiten des BMBF veröffentlicht (Internetquelle 12). Von den Zielen, dem Inhalt und den Ergebnissen des Workshoptages berichtet im vorliegenden Band zudem der Beitrag von Dr. Friderike Lange „Feedback als Haltung in der Lehrerinnenbildung - im Spannungsfeld zwischen Beraten und Bewerten“.

\section{Podiumsgespräch „Visionen und Grenzen}

der neuen digitalen Kommunikationswelt 4.0“

Die hallesche Sprechwissenschaft arbeitet und forscht seit langem in diversen Kooperationen und Kontexten zur Kommunikationsoptimierung und Digitalisierung. Um die Ergebnisse dieser Zusammenarbeit und neue Forschungsprojekte vorzustellen sowie gemeinsam nach Antworten auf die herausfordernden Fragen der digitalen mündlichen Kommunikation zu suchen, wurde am 17. Januar 2020 in die „Händelhalle“ der Stadt Halle zu einem öffentlichen Podiumsgespräch zum Thema „Visionen und Grenzen der neuen digitalen Kommunikationswelt 4.0“ eingeladen. Es kamen neben interessierten Bürger*innen vor allem Kooperationspartner*innen aus Wirtschaft, Wissenschaft und Forschung, die die Sprechwissenschaft als Netzwerkpartnerin in den unterschiedlichen Anwendungsfeldern, wie Kund ${ }^{\star}$ innendienstleistung, digitale Gesundheitsversorgung, IT-technische bzw. KI-basierte Kommunikationslösungen sowie der IT-gestützten Bildung ansehen. Besonders hervorzuheben ist hier der Kontakt zu Prof. Matthias Hagen (Professor für Big Data Analytics an der MLU). Mit ihm wird eine gemeinsame Forschungsarbeit zwischen der sprechwissenschaftlichen Rhetorikforschung (insbesondere Argumentation) und den Big Data Analysen angestrebt. Ebenso konnte der Kontakt zum Bündnismanagement des vom BMBF geförderten TDG-Projektes (Internetquelle 13) intensiviert und dabei die jüngsten Forschungsbemühungen der Sprech- 
wissenschaft bei der Digitalisierung der Gesundheitsversorgung (insbes. der Sprachtherapie) herausgestellt werden. Zwei Beiträge im vorliegenden Band von Prof. Dr. Baldur Neuber „Miteinander reden im digitalen Kontext“ sowie Dr. Judith Pietschmann und Dr. Alexandra Ebel „Visionen und Grenzen der digitalen Kommunikationswelt 4.0 aus sprechwissenschaftlicher Perspektive“ thematisieren die Chancen und Grenzen einer zunehmen digitalen, KI- und roboterbasierten Mensch-Maschine-Mensch-Kommunikation.

\section{Internationales Arbeitstreffen}

\section{„Kinder im Gespräch - Mit Kindern im Gespräch“}

Am 24. und 25. Januar 2020 fand das Internationale Arbeitstreffen „Kinder im Gespräch - Mit Kindern im Gespräch“ von Wissenschaftlerinnen statt, die das Argumentieren in Gesprächen mit Kindern untersuchen. Im Rahmen der öffentlichen Tagung wurden neben Fachvorträgen zur Pädagogik der frühen Kindheit und zum Argumentieren bei Kindern auch Wissenschafts-Praxis-Kooperationen der Sprechwissenschaft mit halleschen Kitaträgern vorgestellt, die seit über 10 Jahren bestehen. Es wurden ebenso methodische Zugänge zu gewachsenen Qualifizierungsmaßnahmen beleuchtet wie wissenschaftliche Erkenntnisse ausgetauscht. Es kamen ca. 80 Wissenschaftler*innen, Vertreter*innen von Kitas und ihren Trägern sowie des Jugendamtes Halle zum Erfahrungsaustausch und zur Planung weiterer Kooperationen zusammen. Gerade dieser Austausch wurde von den Beteiligten als besonders wertvoll geschätzt.

Es folgte dann ein Arbeitsgruppen-Workshop mit 19 Forscherinnen und Forschern aus Deutschland, Norwegen und der Schweiz, die alle interaktionsanalytisch an authentischen Daten zur Kinderkommunikation arbeiten. In einem wissenschaftlichen Austausch über theoretische Positionen, methodische Zugänge und aktuelle Projekte hat das Forscherteam Erkenntnisse für den Transfer in die frühpädagogische Praxis gesammelt. Über die Wissenschafts-Praxis-Kooperationen der Sprechwissenschaft mit halleschen Kitaträgern und die gemeinsamen Forschungsergebnisse berichten Prof. Dr. Ines Bose, Dr. Stephanie Kurtenbach, Franziska Kreutzer und Kerstin Kreße im Beitrag „Mit Kindern im Gespräch. Sprechwissenschaftliche Kita-Forschung mit Praxiskooperationen“. 


\section{Feierliche Abschlussveranstaltung: Textwerkstatt „Ein Tag zum Geschenk“ und Video-Premiere „Einar-Schleef-Projekt der Sprechbuehne“}

Am 31. Januar 2020 fand dann die feierliche Abschlussveranstaltung der Kleine Fächer-Wochen Sprechwissenschaft an der MLU statt.

Im ersten Teil präsentierten die Studierenden des 2. Bachelor-Studienjahrs Sprechwissenschaft einen öffentlichen Sprechkunstabend als Werkstattbericht zum Thema „Ein Tag zum Geschenk“. Die Studierenden führten das Publikum literarisch durch einen imaginären Tag, einen geschenkten Tag, denn das Jahr 2020 war ein Schaltjahr (Internetquelle 14). Mit Texten aus Lyrik, Prosa, Dramatik und Wissenschaft zeigten sie, wie sich ein Liebhaber Anno 1905 aus Liebchens Paradies stahl, wie Kafka die kleine Geste des über die Augenbraue-Streichen beschreibt und welche positiven Auswirkungen das Küssen hat. Auf sehr ein- und nachdrückliche Weise bewiesen die Studierenden ein feines sprechkünstlerisches Gespür und konnten ihre bisher erlernten Fähigkeiten voll zum Ausdruck bringen. Diese Präsentation war nicht nur für das hallesche Publikum ein unvergesslicher Moment mit einem ganz besonderen Zauber.

Den Abschluss der „Kleine Fächer-Woche Sprechwissenschaft“ bildete dann die öffentliche Video-Premiere „Einar-Schleef-Projekt der Sprechbuehne" (Internetquelle 15). Das Projekt entstand in Zusammenarbeit mit dem Kunstmuseum Moritzburg Halle (Saale), das den bildkünstlerischen Nachlass des Künstlers verwaltet. Anlässlich des 75. Geburtstages Schleefs (1944-2001) hatte ihm die "Sprechbuehne“ (Leitung: Dr. Martina Haase) eine szenische Sprechcollage "Ich bin ein anderer in mir, den muss ich fragen. Einar Schleef“ gewidmet, die im Rahmen einer Sonderausstellung des Kunstmuseums Moritzburg 2019 mehrmals zur Aufführung kam. Der Öffentlichkeit konnte durch die Video-Premiere und das nachfolgende Gespräch mit Susanna Köller, Kustodin der Grafischen Sammlung des Kunstmuseums Moritzburg, Hans-Ulrich Müller-Schwefe, langjährigem Freund und Lektor von Schleef, Suhrkamp Verlag, Dr. Martina Haase und Andreas Montag (Moderation), Ressortleiter Kultur der Mitteldeutschen Zeitung ein Eindruck vom vielfältigen künstlerischen Schaffen des genialischen, vielseitigen Künstlers, Theaterregisseurs, Autors und Malers Schleef gegeben werden. Von der Zusammenarbeit berich- 
tet der Beitrag „Die Sprechbuehne und das Kunstmuseum Moritzburg Halle (Saale) - Geschichte einer Kooperation“ von Dr. Martina Haase und Thomas Bauer-Friedrich, dem Direktor des Museums.

\section{Resümee der Kleine Fächer-Wochen Sprechwissenschaft an der Universität Halle}

Die KFW der halleschen Sprechwissenschaft zeigten einen großen Effekt und brachten einen enormen Zugewinn für das Fach. Sie unterstützten die fachliche Standortbestimmung und verhalfen zu neuen interdisziplinären Kooperationsmöglichkeiten und Forschungsfeldern.

Durch eine moderne Kommunikation, aber vor allem durch aktivierende und auf Partizipation aller Akteure, Teilnehmer*innen und Partner*innen ausgerichtete Formate konnte sich die Sprechwissenschaft den alten und neuen Kooperations- und Praxispartner*innen in Forschung, Politik, Kultur, Medizin und Gesellschaft sowie all den Nutznießer*innen in ihrer fachlichen Breite und Spezifizität präsentieren. Gegenüber der allgemeinen Öffentlichkeit konnte damit die gesellschaftliche und Alltagsrelevanz der Sprechwissenschaft noch sichtbarer gemacht werden. Die gesellschaftlichen Bedürfnisse an das Fach traten deutlich hervor und die sprechwissenschaftliche Expertise wird seitdem noch stärker nachgefragt.

Das Potenzial der Sprechwissenschaft in Lehre und Lernen konnte durch die Präsentation des spezifischen Kompetenz- und Wissenserwerb und die sich damit eröffnenden vielfältigen beruflichen Perspektiven, z. B. im rhetorischen, sprechkünstlerischen, (früh-)pädagogischen, sprachtherapeutischen, DaFoder Coaching/Beratungs-Bereich aufgezeigt werden. Es wurde fach- und zielgruppenspezifisch dargestellt, welche wissenschaftlichen Qualifikationswege das gesamte Bachelor- und Masterstudium der Sprechwissenschaft eröffnet. Durch die Präsentation der Dissertations- und Forschungsprojekte zeigte sich dabei erneut die wissenschaftliche Exzellenz der Sprechwissenschaft. Sie besitzt zudem ein enormes Vernetzungspotenzials und agiert das bereits in verschiedenen interdisziplinären Forschungs- und Lehrverbünden aus. Das zeigt sich auch in ihrer nationalen, internationalen und institutionellen Zusammenar- 
beit mit anderen, größeren Disziplinen, wie der Sprachwissenschaft, Medizin, Informatik oder Pädagogik.

Die Sprechwissenschaft ist anwendungsbezogene und lebenswirkliche Disziplin, die zu den Lebenswissenschaften zählt. Durch ihr wissenschaftliches und praktisches Wirken für ein verantwortliches, kompetentes, reflektiertes Miteinandersprechen der Menschen wird ein essenzieller Beitrag für den gesellschaftlichen Fortschritt und Zusammenhalt geleistet. Dieser hängt letztendlich auch davon ab, wie verantwortlich, kompetent und reflektiert die einzelnen Menschen (miteinander-)sprechen bzw. dies erlernen können und wie ihre individuellen Bedürfnisse im Gespräch Berücksichtigung finden. Das Bedarf vor allem Zeit und Aufmerksamkeit. Diese Zeit für einen erneuten gesellschaftlichen und interdisziplinären Austausch ermöglichten die „Kleine Fächer-Wochen Sprechwissenschaft" in Halle. Sie lenkten die Aufmerksamkeit auf die sprechwissenschaftlichen Forschungs-, Lehr- und Anwendungsfelder sowie ihrer Transferstärke in und für die verschiedensten gesellschaftlichen Bereiche.

Alle Presseberichte (Internetquelle 16) sowie der Blogbeitrag auf den Seiten der HRK (Internetquelle 17) zur KFW Sprechwissenschaft in Halle sind nachzulesen.

Den KFW Sprechwissenschaft vorangestellt war ein Workshop „Social Media in der Sprechwissenschaft" von Tim Schwiesau, der zur Professionalisierung der Wissenschaftskommunikation der Abt. Sprechwissenschaft und Phonetik an der MLU beigetragen hat. So wurden aus verschiedenen Veranstaltungen der KFW SW Beiträge auf dem Instagram-Profil (Internetquelle 18) https://www.instagram.com/sprechwissenschaft.halle/?hl=de und auf dem Facebook-Account (Interquelle 19) gepostet. 


\section{Literatur}

Voigt-Zimmermann, Susanne/Pietschmann, Judith (2020): Die Kunst des „Miteinandersprechens" - eine sprechwissenschaftliche Perspektive. In: Die politische Meinung. Berlin: Konrad-Adenauer-Stiftung, 65(562), S. 49-55.

\section{Internetquellen}

Internetquelle 1: https://kfw.sprechwiss.uni-halle.de (Letzter Zugriff: 24.02.2021)

Internetquelle 2: https://www.youtube.com/watch?v=h95Q6KDkQi4

(Letzter Zugriff: 24.02.2021)

Internetquelle 3: https://www.campus-halensis.de/artikel/kleine-facher-wochensprechwissenschaft-sie-mussen-sich-halle-merken/ (Letzter Zugriff: 24.02.2021)

Internetquelle 4: https://hallespektrum.de/nachrichten/ausstellung-wassprachtherapie-kann-im-rathaus/360163/ (Letzter Zugriff: 24.02.2021)

Internetquelle 5: https://www.youtube.com/watch?v=d_teNR6ZH3c\&feature=emb_ logo (Letzter Zugriff: 24.02.2021)

Internetquelle 6: https://ondemand-mp3.dradio.de/file/dradio/2019/11/28/ neues_beratungsangebot_sprechwissenschaften_in_halle_dlf_20191128_1454_ 96658338.mp3 (Letzter Zugriff: 24.02.2021)

Internetquelle 7: https://kfw.sprechwiss.uni-halle.de/sprechideale-und-idole-auf-derbuehne-in-geschichte-und-gegenwart-musik-und-sprechwissenschaftlicheaspekte/ (Letzter Zugriff: 24.02.2021)

Internetquelle 8: https://kfw.sprechwiss.uni-halle.de/oeffentliche-ringvorlesungideale-in-musik-gesang-medien-und-sprechen/ (Letzter Zugriff: 24.02.2021)

Internetquelle 9: https://www.campus-halensis.de/artikel/sprechen-ohne-zu-schreien/ (Letzter Zugriff: 24.02.2021)

Internetquelle 10: https://kfw.sprechwiss.uni-halle.de/sprechkuenstlerischerworkshop-chorisches-sprechen-mit-bernd-freytag/ (Letzter Zugriff: 24.02.2021)

Internetquelle 11: http://vap.sprache-interaktion.de/ (Letzter Zugriff: 24.02.2021)

Internetquelle 12: https://www.qualitaetsoffensive-lehrerbildung.de/de/feedbackkulturin-der-lehrer-innenbildung---ein-praxisorientierter-workshop-tag-in-halle2264.html (Letzter Zugriff: 24.02.2021)

Internetquelle 13: https://inno-tdg.de/ (Letzter Zugriff: 24.02.2021)

Internetquelle 14: https://kfw.sprechwiss.uni-halle.de/files/2020/01/FlyerEinTagzumGeschenk.pdf (Letzter Zugriff: 24.02.2021)

Internetquelle 15: https://sprechbuehne.uni-halle.de/ (Letzter Zugriff: 24.02.2021)

Internetquelle 16: https://kfw.sprechwiss.uni-halle.de/die-kleine-facher-wochen-dersprechwissenschaft-in-den-medien (Letzter Zugriff: 24.02.2021) 
Internetquelle 17: https://www.hrk.de/themen/hochschulsystem/kleine-faecher/ kleine-faecher-wochen-an-deutschen-hochschulen/blogbeitraege/beitrag-vom27112019/ (Letzter Zugriff: 24.02.2021)

Internetquelle 18: https://www.instagram.com/sprechwissenschaft.halle/?hl=de (Letzter Zugriff: 24.02.2021)

Internetquelle 19: https://de-de.facebook.com/Sprewi/?ref=page_internal (Letzter Zugriff: 24.02.2021) 


\title{
Sichtbar(er) werden: Die Kleine Fächer-Wochen in Marburg und die Sprechwissenschaft
}

Zusammenfassung: Dieser Essay stellt die Kleine Fächer-Wochen an der Universität Marburg vor und blickt auf den Anteil der Sprechwissenschaft und die Wirkung, die dieses Förderprogramm auf die Sprechwissenschaft in Marburg gehabt hat. Auf dieser Grundlage werden ich einige Positionen für die Zukunft des Faches, nicht nur in Marburg, formulieren. Wenn wir wollen, so glaube ich, kann diese Initiative und die Reflexion über die Sprechwissenschaft als Kleines Fach als Katalysator zur Entwicklung des Faches dienen.

Stichworte: Mündlichkeit, Stimmlichkeit, Internationalisierung, Kleine Fächer

\begin{abstract}
This essay presents the Small-Disciplines-Weeks at the University of Marburg with a special focus on the part speech communication played in it and the effect it has had on speech communication at Marburg in the long run. Starting from this, I shall map out some aspects for the future of this small discipline. The initiative of the Small-Disciplines-Weeks can lead to a clear reflection of speech communication as a very specific small discipline and can function as a catalyst in its development.
\end{abstract}

Keywords: Orality, voice, internationalization, small disciplines

Dieser Essay stellt die Kleine Fächer-Wochen an der Universität Marburg vor und blickt auf den Anteil der Sprechwissenschaft und die Wirkung, die dieses Förderprogramm auf die Sprechwissenschaft in Marburg gehabt hat. Auf dieser Grundlage werden ich einige Positionen für die Zukunft des Faches, nicht nur in Marburg, formulieren. Wenn wir wollen, so glaube ich, kann diese Initiative und die Reflexion über die Sprechwissenschaft als Kleines Fach als Katalysator zur Entwicklung des Faches dienen. 


\section{Die weite Welt vor Ort}

Seit den Kleinen Fächer-Wochen ist in Marburg deutlich seltener zu hören „Ach, Sprachwissenschaftlerin sind Sie?“ oder „Oh, Sprechwissenschaft. Ist das so was wie Logopädie?“. Die Kleine Fächer-Wochen an der Philipps-Universität Marburg, gefördert durch die HRK, war ein gemeinsames Projekt der kleinen geistes- und sozialwissenschaftlichen Fächer. Im Zentrum stand die herausragende Rolle, die kleine Fächer für die Internationalisierung spielen. Dadurch, dass unsere engeren fachlichen Gesprächspartner_innen häufig nicht auf dem nächsten Flur sitzen, sind Kleine Fächer oft international ausgerichtet. Diese internationale Ausrichtung ist in der Regel so selbstverständlich, dass weder das Fach noch die Universität dieses Potenzial der Kleinen Fächer herausstellt. Die Kleine-Fächer Woche sollte daher unter dem Motto: Die weite Welt vor Ort. Der Beitrag der Kleinen Fächer zur Internationalisierung der Philipps-Universität Marburg. (https://www.uni-marburg.de/de/international/ profil/projekte/kleine-faecher-wochen) diese besondere Rolle herausstellen.

Die Sprechwissenschaft als Kleines Fach war an drei Positionen dieses Projektes präsent: Als eigenes Fach, das sich an Ausstellungen, Diskussionsrunden, Messetag, etc. beteiligte, als ein Kleines Fach am Institut für Germanistische Sprachwissenschaft unter insgesamt fünf Kleinen Fächern und als Leitung des Gesamtprojektes, die bei mir lag.

\section{Die Sprechwissenschaft als Kleines Fach in Marburg}

Die Sprechwissenschaft ist an Universitäten in Deutschland unterschiedlich aufgestellt und angebunden. Anders als viele sprechwissenschaftlich-sprecherzieherischen Einheiten, die keine eigenen Studiengänge anbieten und als Prüfstellen der DGSS fungieren, ist die Sprechwissenschaft an den Universitäten Halle, Jena und Marburg und an der Hochschule für Musik und Darstellende Kunst in die Fakultäten/Fachbereiche eingebunden (vgl. auch Hannken-Illjes 2019). In Marburg ist die Sprechwissenschaft - wie in Jena - eine Arbeitsgruppe im Institut für Germanistische Sprachwissenschaft und besteht aus der Professur und derzeit drei Mitarbeiterinnen. Damit ist die Sprechwissen- 
schaft in Marburg klar in der Germanistik und in der Linguistik verortet. Die Forschungsschwerpunkte, die in Marburg derzeit bearbeitet werden, sind, die Herstellung von Verstehen und Relevanz in der Schwangerenberatung, die Argumentationsfähigkeit von Vorschulkindern (gemeinsam mit Ines Bose und Stephanie Kurtenbach in Halle), die Verbindung von Narration und Argumentation und Vermittlungsformen von Wissen zwischen Kunst und Wissenschaft (gemeinsam mit Eva Maria Gauss). Daneben gibt es acht laufende Promotionen. In der Lehre ist die Sprechwissenschaft fest in den Curricula im Fach Deutsch im Lehramtsstudium und im Bachelor Sprache und Kommunikation verankert und verantwortet gemeinsam mit der Phonetik einen Masterstudiengang in Sprechwissenschaft und Phonetik.

Interessant an der Anbindung im Institut für Germanistische Sprachwissenschaft ist die besondere Marburger Situation. Die Universität Marburg verfügt über eine außerordentlich große, ausdifferenzierte Germanistische Sprachwissenschaft mit acht Professuren und zwei weiteren Professuren am Zentrum Deutscher Sprachatlas. In der Vorbereitung der Kleine Fächer-Woche wurde zudem klar, dass im Institut für Germanistische Sprachwissenschaft neben den „großen“ auch fünf Kleine Fächer zusammenkommen: Deutsch als Fremdsprache, Klinische Linguistik, Neurolinguistik, Phonetik und Sprechwissenschaft.

Hier zeigt sich, dass die Selbstidentifizierung als Kleines Fach innerhalb des Instituts und auch innerhalb der Universität sehr unterschiedlich ausgeprägt war und auch nicht von allen positiv gesehen wurde. So haben sich einige nicht als eigene Fächer wahrgenommen, sondern ganz klar als Teil eines größeren Fachs, zum Beispiel der Germanistischen Sprachwissenschaft, andere fanden sich gar nicht so klein. Hier hatte die Initiative der Hochschulrektorenkonferenz schon vor Projektbeginn einen wichtigen Impuls: Die Reflexion innerhalb des eigenen Faches wodurch es sich, neben den klaren Kriterien wie Studiengängen und Publikationsorten, als eigenes Fach auszeichne. Unter dem Dach „Kleines Fach" kommt eine heterogene gruppe von Disziplinen zusammen, die unter sehr unterschiedlichen Bedingungen arbeiten; manche gerade noch ein Fach, manche schon fast nicht mehr klein, manche von ihrer gesamten Anlage ein stabile, aber Kleines Fach. Für die Sprechwissenschaft die sich immer schon klar als eigenes Fach definiert hat haben sich eher andere Fragen gestellt und 
sie stellen sich noch: Wir haben eigene Studiengänge und Professuren mit der Denomination „Sprechwissenschaft“ - wenn auch nicht viele, doch dazu später - aber wir haben beispielsweise keine eigene forschungszentrierte Zeitschrift und eine wissenschaftliche Gesellschaft, die in Teilen ein Berufsverband ist. Das ist zum einen typisch für Fächer mit einem starken Praxisbezug, zum anderen schwächt es unsere Position als wissenschaftliche Disziplin.

\section{Die Sprechwissenschaft in den Kleinen-Fächer-Wochen}

Die Sprechwissenschaft als Fach, das auch in Marburg einen starken Fokus auf der Praxis von Mündlichkeit und Stimmlichkeit und auf die Anwendungsorientierung der Forschung hat, hat auf den Kleine Fächer-Wochen in Marburg vor allem Veranstaltungen zur Berufsorientierung mitverantwortet und unterstützt; zwei sollen hier skizziert werden.

Der Auftakt der Marburger Kleine Fächer-Wochen am 5. November 2019 stand unter der Überschrift „Kleine Fächer studieren: Kompetenzen und Chancen für den internationalen Arbeitsmarkt" und verband eine Keynote von Prof. Dr. Markus Hilgert, Generalsekretär der Kulturstiftung der Länder und Alumnus der Universität Marburg, mit einer Podiumsdiskussion auf der ich als Sprechwissenschaftlerin teilnahm. Diskutiert wurde unter anderem das Problem der Kleinen Fächer, gut zu kommunizieren, was man „mit ihnen machen kann“, da Schülerinnen und Schüler die Kleinen Fächer eben nicht aus dem Schulunterricht kennen und so möglicherweise kein klares Bild von Gegenständen und Berufsperspektiven haben. Was nicht heißt, dass die großen Fächer nicht möglicherweise oft ein Problem damit haben, dass Schülerinnen und Schüler ein vermeintlich klares Bild eines Faches haben und dann feststellen, dass das wissenschaftliche Fach sich vom Schulfach deutlich unterscheidet.

Die fünf Kleinen Fächer der Germanistischen Sprachwissenschaft organisierten eine gemeinsamen Messetag für Studieninteressierte: Mitmachstationen mit Kehlkopfultraschall, Argumentationstraining, Stimmübungen, EEG, Sprachtests und den Auftritten des Beatboxers Babeli und eines forensischen Phonetikers der lange Zeit beim Bundeskriminalamt gearbeitet hatte zog erstaunlich viele Interessierte an und fand reichen Niederschlag in der lokalen Pressen. 
Zudem war die Sprechwissenschaft an einer Reihe von Veranstaltungen beteiligt, gemeinsam mit allen oder einigen anderen Kleinen Fächern: Wir waren Teil der Ausstellung zu den Kleinen Fächern in Marburg, haben mit einer Sprechwissenschaftlerin aus Halle, Dr. Friderike Lange, die interdisziplinäre Vortragsreihe zur Berufsorientierung bereichert, haben an Veranstaltungen für Schülerinnen und Schüler teilgenommen und haben - natürlich - mit den Kolleg_innen aus der Phonetik die Internationalisierung Kleiner Fächer hörbar gemacht (Ratibor! Und der Fluss Mississippi und die Stadt Honolulu...).

\section{$4 \quad$ Internationalisierung}

Die Verbindung der 24 sozial- und geisteswissenschaftlichen Kleinen Fächer der Universität Marburg stand unter der Überschrift „Die weite Welt vor Ort“ und damit der Internationalisierung der Universität und den Beitrag den die Kleinen Fächer daran haben. Möglicherweise weniger sichtbar als bei großen Fächern, tragen Kleine Fächer oft automatisch zur Internationalisierung bei, da die Kolleginnen und Kollegen im Fach nicht an der eigenen Universität zu finden sind und so das fachliche Gespräch selbstverständlich über Ländergrenzen hinweg geführt wird.

Stimmt das auch für die Sprechwissenschaft? Wie sieht es mit der Sprechwissenschaft in Marburg aus, wie international ist sie? Ist die Forschung international verortet? Sicher, das ist sie und das fachliche Gespräch findet selbstverständlich über die Grenzen der Nationalstaaten hinweg statt; auf Tagungen und in Zeitschriften zur Rhetorik, zur Argumentation, zur Narration, zur Interaktion von Kindern. Die interessantere Frage ist aber, ob diese Arbeit als sprechwissenschaftliche Arbeit sichtbar ist? Oder nicht vielmehr als argumentationswissenschaftliche, rhetorische, allgemein linguistische oder mikrosoziologische mit Interesse an Sprache und Kommunikation? Wenn ich einen methodologischen Aufsatz zur ethnography of argumentation (Hannken-Illjes 2020) schreibe, ist das dann ein sprechwissenschaftlicher Aufsatz? Es ist in jedem Fall nicht ein nicht-sprechwissenschaftlicher Aufsatz und es ist für die community der Argumentationswissenschaft gänzlich unerheblich, ob 
ich Linguistin bin oder Sprechwissenschaftlerin oder Philosophin. Fraglich ist, ob es für unser Fach auch unerheblich ist.

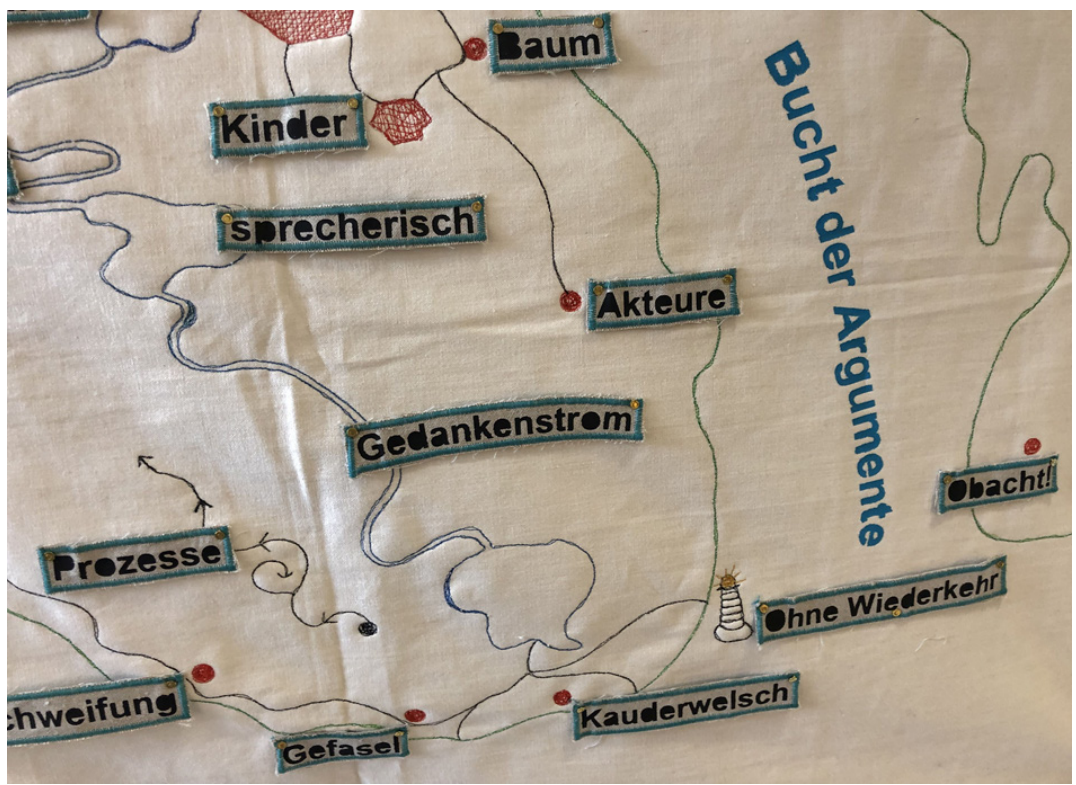

Abb. 1: Collage von Eva Filipski zur Argumentation und Sprechwissenschaft (Foto: Kat Hannken-Illjes)

Hier kommt noch ein Aspekt hinzu: International identifiziere ich mich in der Regel als Vertreterin der speech communication manchmal als Rhetorikerin, nie als Linguistin. Damit referiere ich auf das Profil unserer Schwesterdisziplin in den USA das heute selbst nicht mehr unter speech communication sondern communication studies firmiert. Doch auch wenn diese Disziplinen aus den gleich Impulsen zu Beginn des 20. Jahrhunderts entstanden sind (vgl. Gronbeck 2005), so haben sie sich doch unterschiedlich entwickelt. Die communication studies sind hochausdifferenziert, mit verschiedensten Publikationsorganen, verschiedenen methodologischen Strömungen und Gegenständen, mit Studienprogrammen an den meisten US-Universitäten, Forschungsuniversitäten ebenso wie kleineren Colleges. Beinhaltet in dieser Selbstpositionierung ist die Identifizierung als rhetorician als traditionell einem Teil der communication 
studies. Mit dieser Positionierung bin ich Teil einer Wissenschaftscommunity, die sich unter anderen auf der Konferenz der Rhetoric Society of Europe, der International Society for the Study of Argumentation und der European Conference for the Studie of Argumentation trifft. Dies ist eine völlig andere Ausrichtung als die der Sprechwissenschaft in Halle oder Jena oder Stuttgart. Ist das ein Problem? Ich glaube nicht. Nutzen wir diese Diversität als Fach schon ausreichend? Auch das glaube ich nicht.

\section{Lessons learned: Die Perspektive des Sprechwissenschaft als Kleines Fach}

Die Kleine Fächer-Wochen haben der Sprechwissenschaft an vielen Standorten Sichtbarkeit verschafft, zu Vernetzung mit anderen Fächern geführt und so Wind unter die Flügel gebracht. Sie kann aber kurzfristig nicht die strukturellen Bedingungen ändern unter denen die Sprechwissenschaft arbeitet. Die Sprechwissenschaft ist in Marburg auf professoraler Ebene - wie in Stuttgart und Jena - eine one-(wo)man-show. Wie kann unter diesen Bedingungen die Entwicklung dieses Kleinen Faches aussehen?

\section{Wir müssen Schwerpunkte setzen}

Die Sprechwissenschaft in Marburg hat eine klare Schwerpunktsetzung in der Lehre und in der Forschung: Rhetorik und ästhetische Kommunikation unter den Bedingungen von Mündlichkeit und Stimmlichkeit. In der Lehre wird in erster Linie auch die Didaktik der Rhetorik vermittelt, daneben aber auch Rhetorische Analyse und natürlich Seminare zu aktuellen Forschungsfragen. Hinzu kommt ein methodologischer Schwerpunkt auf qualitative Methoden. Aus meiner Sicht liegt hier die klare Entwicklungsperspektive der Sprechwissenschaft im deutschsprachigen Raum: Mut zur Schwerpunktsetzung und Verzicht auf den Anspruch, an einem Standort die gesamte Breite des Faches anzubieten von den Grundlagen bis zu aktuell relevanter Forschung. Sprechwissenschaft in Marburg ist in erster Linie mündliche Rhetorik. 


\section{Die gemeinsamen Grundlagen sichern}

Diese Differenzierung funktioniert nur, wenn dennoch eine gemeinsame Position der Sprechwissenschaft festgehalten wird: zur Gegenstandskonstitution und zum Grundverständnis des Faches, was den Status der Praxis, der Didaktisierung und der Anwendung betrifft. Hier haben wir mit Mündlichkeit und Stimmlichkeit eine Schnittmenge, aber eine Schnittmenge die selbstverständlich immer auch in andere, auch große, Fächer führt. Nun könnte man anführen, dass unsere Gemeinsamkeit in der Anwendungsorientierung liegt, auch in der didaktischen Perspektive auf Mündlichkeit und Stimmlichkeit. Das scheinen aber viele Forschungsprogramme innerhalb des Faches gar nicht abzubilden. Vielleicht ist stärker als die Anwendungsorientierung, die Einbeziehung der Praxis in das Studium: Wir verleiben uns Theorien ein und reflektieren die Einverleibung dann in Forschung und Theoriebildung.

\section{Kleines Fach - klein stark bleiben}

Die Sprechwissenschaft ist ein Kleines Fach. Blickt man auf die Kriterien der Kartierung Kleiner Fächer des Portals Kleiner Fächer in Mainz, so liegt der Unterschied zwischen einem Kleinen und einem nicht mehr Kleinen Fach an der Anzahl der unbefristeten Professuren an einzelnen Universitäten (nicht mehr als drei an einer einzelnen Universität) und über alle Universitäten hinweg (nicht an mehr als $10 \%$ der deutschen Universitäten professoral verankert). Es steht nicht zu erwarten, dass die Sprechwissenschaft mittelfristig an mehr als 12 Universitäten Professuren haben wird, auch nicht, dass in Halle, Marburg oder Jena auf längere Sicht vier oder mehr Professuren für Sprechwissenschaft etabliert werden.

Statt anzustreben, groß zu werden, sollten wir damit weitermachen, stark zu werden: Sichtbare Teile unserer Universitäten und der Forschungslandschaft. Bei der Sichtbarkeit hat uns die Kleine Fächer Woche geholfen, für alles andere sind wir zuständig. 


\section{Literatur}

Gronbeck, Bruce (2005): Is Communication a Humanities Discipline? Struggles for Academic Identity. In: Arts and Humanities in Higher Education, 4, 3, 229-246. Hannken-Illjes, Kati (2019): Kleine Fächer im Porträt: Sprechwissenschaft. https:// www.kleinefaecher.de/beitraege/blogbeitrag/sprechwissenschaft.html, (letzter Zugriff 01.09.2020)

Hannken-Illjes, Kati (2020): Ethnography of Argumentation. In Proceedings oft the Third European Conference on Argumentation, Leiden UP, 251-268. 

Kerstin Schuck, Jena

Ein kleines, aber kräftiges Gewächs:

Die schöne Blume „scientia orationis“ aus Jena

Zusammenfassung: Die Friedrich-Schiller-Universität Jena machte mit zahlreichen Veranstaltungen zu den „Kleine Fächer-Wochen“ auf die gesellschaftlich-kulturelle Relevanz der vielfältigen Studienangebote aufmerksam. Der interdisziplinäre Studiengang „Sprechwissenschaft und Phonetik“ ist eines von insgesamt 21 „kleinen“ Studienfächern an der Philosophischen Fakultät. In diesem Artikel werden die Veranstaltungen dieses Studienfachs zur „Kleine Fächer-Woche“ vorgestellt.

Stichworte: Sprechwissenschaft, Phonetik, Fach mit vielen Stimmen, Absolventen, Forschungsprojekte

Abstract: Various events which took place within the "small disciplines weeks" of the Friedrich-Schiller-University Jena draw attention to the socio-cultural relevance of a diverse range of studies. The interdisciplinary study of „Speech Science and Phonetics“ is one of 21 „small" study courses offered at the faculty of philosophy. In this article the events performed of this discipline within the "small subject weeks" are presented.

Keywords: Speech Science, Phonetics, Subject with many voices, Alumni, Research projects

„Alles Orchideen?“ Mit diesem Titel wurde an der Friedrich-Schiller-Universität (FSU) in Jena für die Veranstaltungsreihe „Kleine Fächer-Wochen“ geworben, welche über die Dauer des Wintersemesters 2019/2020 stattfand.

Als „Orchideen-Fächer" werden im Allgemeinen solche Studienfächer bezeichnet, welche eher ausgefallen sind und in der Regel nur von wenigen Studierenden gewählt werden. Der Verweis auf die Botanik drückt aus, dass diese außergewöhnlichen Pflanzen nicht nur von KennerInnen geschätzt werden, sondern dass sie aufgrund ihres seltenen Vorkommens schützenswert sind. 
An der Philosophischen Fakultät sind dies immerhin 21 „kleine“ Studienfächer. Das umfasst mehr als ein Viertel der dort angebotenen Fächer und die Sprechwissenschaft und Phonetik ist eines davon. Der Blick der Öffentlichkeit wurde durch verschiedene Veranstaltungen wie Thementage, Vorträge, Ausstellungen, Performances und Filmvorführungen wie auch Workshops auf diese Orchideen gelenkt.

Das Ziel der Veranstaltungswochen lag in der Sichtbarmachung der vielfältigen Studierangebote und wies durch die konkrete Vorstellung der „Kleinen Fächer" mit ihren Studieninhalten und Perspektiven auf deren gesellschaftliche und kulturelle Relevanz hin. Die breite Fächerlandschaft der FSU und die damit verbundenen Auswahlmöglichkeiten werden auch von Fächern wie beispielsweise Klassische Archäologie, Gräzistik, Kaukasiologie, Indogermanistik, Filmwissenschaft, Orientalistik und Latinistik bereichert.

Auch die Sprechwissenschaft und Phonetik beteiligte sich mit einem breit gefächerten Angebot am Veranstaltungskanon. Präsentiert wurden sprechkünstlerische Programme, Vorträge zu aktuellen Forschungsfeldern der Professur, eine Podiumsdiskussion sowie ein interaktives Programm zur Sichtbarmachung des Sprechvorgangs. Nachfolgend werden die einzelnen Veranstaltungsangebote näher beschrieben.

Das von Studierenden der Sprechwissenschaft gestaltete und gesprochene Programm „Farbe auf die Ohren“wurde am 16.01.2020 im Café Wagner in Jena aufgeführt und lud dazu ein, den grauen Alltag zu vergessen. Die rezitierten Texte über das Thema „Farben“ beinhalteten sowohl Erzählungen von Autoren wie Ephraim Kishon, Drew Daywalt und Oliver Jeffers, Lyrik von Arno Holz und Erich Kästner sowie von den Studierenden selbst verfasste Prosatexte. Ausdrucksvoll und lebendig erzählten sie von nicht ganz alltäglichen Vorfällen. Der Einsatz der Bühnentechnik, farbig abgestimmtes Scheinwerferlicht, unterstützte die einzeln oder in der Gruppe vorgetragenen Texte. Unter Einbezug einer dialogisch-interaktiven Methode wurde auch das Publikum mit kurzen Sprechbeiträgen am Programm beteiligt. Dargebracht wurde das Programm auf der hauseigenen Bühne des Cafés. Der von Studierenden und Interessierten besuchte Programmabend wurde als „lohnenswert“ oder „Die Sprewis sind immer für Überraschungen gut“ zusammengefasst. 
Sprechkünstlerisch aktiv zeigten sich nicht nur die Studierenden des Fachs. Die Jenaer DozentInnen präsentierten zur Eröffnungsveranstaltung ein Programm in Form eines Sprechchors, in welchem die Inhalte der Sprechwissenschaft und Phonetik thematisiert und rhythmisch artikuliert wurden. Der Titel „Glottis Rock“ war insofern programmatisch.

In einem interaktiven Programm zur „Langen Nacht der Wissenschaften“ konnten Besuchende am 22.11.2019 an verschiedenen Stationen dem Vorgang des Sprechens auf die Spur kommen. Angeboten wurden sowohl die Verfahren der statischen Palatographie und des Ultraschalls wie auch die Möglichkeit, sich sprechkünstlerisch an verschiedenen Texten von Ernst Jandl zu probieren. Die Veranstaltung wurde von einer Vielzahl von Personen besucht, u.a. von SchülerInnen, Studierenden und Familien.

Am 22.02.2020 lud die Professur zum Infotag „Sprechwissenschaft - ein Fach mit vielen Stimmen" ein, um Studierenden wie auch allen Interessierten die Sprechwissenschaft und Phonetik als Studienfach und ihre vielfältigen Berufsperspektiven vorzustellen. Den Kern der Veranstaltung bildete ein moderiertes Podiumsgespräch mit AbsolventInnen des Fachs, welche derzeit in den Bereichen Forschung, Öffentlichkeitsarbeit, Verwaltung, Kunst, Kultur, Medien oder Bildung tätig sind. In einem belebten Gespräch zwischen Publikum und Alumni wurde thematisch der Einstieg nach dem Studium ins Berufsleben sowie der konkrete Berufsalltag bearbeitet.

Einigkeit herrschte darüber, dass das Studium der Sprechwissenschaft und Phonetik in Jena durch seinen klaren Praxisbezug und aufgrund der Vielfältigkeit der Themen eine sehr gute Vorbereitung für die spätere Berufspraxis darstellt. Hervorgehoben wurde auch der gute Zusammenhalt zwischen den Studierenden und der fördernde Austausch mit den DozentInnen. Viele der AbsolventInnen nehmen das als eine besondere Qualität wahr, welche kleine Fächer leisten können. Die Studierenden erhielten die Möglichkeit, sich im Anschluss persönlich mit den Alumni auszutauschen. Hier wurden Erfahrungswerte aus erster Hand und Eindrücke aus den unterschiedlichsten Berufsfeldern sowie Antworten auf ganz lebenspraktische Fragen für die Zeit nach dem Studium gegeben. Insgesamt bewerteten die Teilnehmenden den Austausch als anregend und aufschlussreich. 
Die aktuellen Forschungsprojekte der Professur wurden anschließend in Form einer Postersession vorgestellt. Die wissenschaftlichen Forschungsfelder umfassen Arbeiten aus dem Bereich der Phonetik, Sprecherziehung wie auch aus der Rhetorik: „Perception of dialectal variation of speakers from East Thuringia“, „Akustisch-phonetische Variabilität in kinder- und erwachsenen-gerichteter Sprache bei Eltern während der frühkindlichen Erziehung“, „Über den Einfluss phonologischer Quantität im Fremdspracherwerb“, „Auswirkungen sprecherischer Auffälligkeiten auf das Hörverstehen im Schulkontext “ und „Reformulierungsverfahren im Mediationsprozess“. Auch hier konnten die BesucherInnen ihre Fragen direkt an die Projektleitenden stellen.
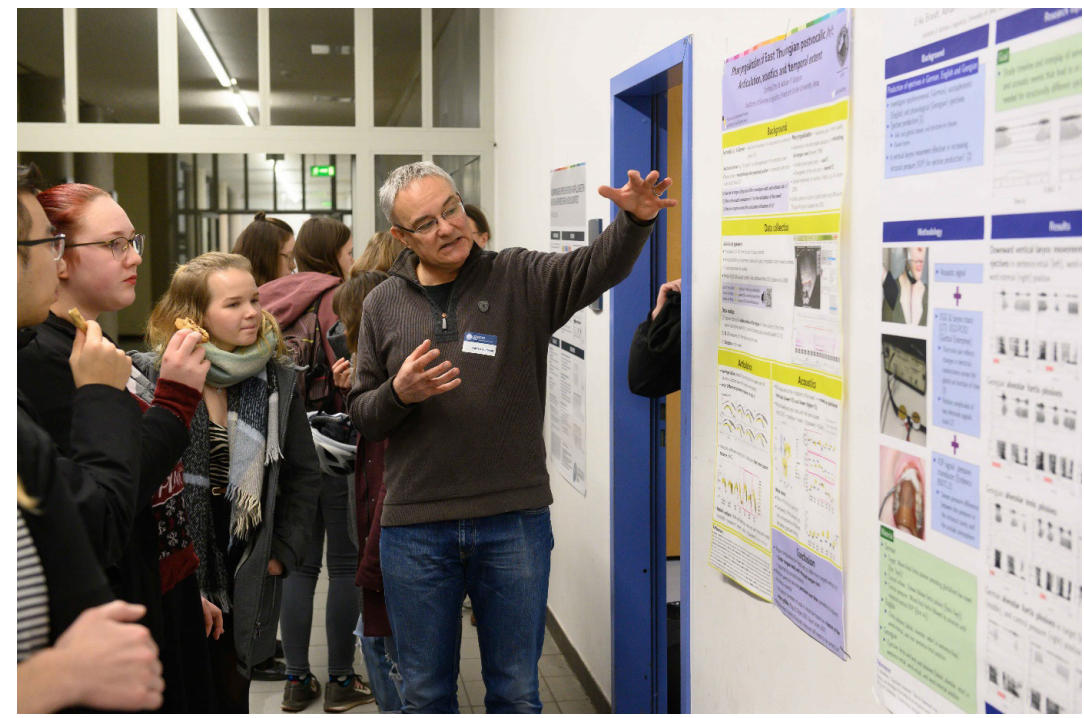

Abb. 1: Professor Simpson mit Studierenden bei der Postersession zum Infotag der Sprechwissenschaft „Ein Fach mit vielen Stimmen“ (Foto: Maik Schuck)

Als Resümee der Kleinen Fächer-Wochen lässt sich festhalten, dass die Sprechwissenschaft und Phonetik auf Resonanz stößt, was an den gut besuchten und mit Interesse aufgenommenen Veranstaltungen wie auch an dem Feedback des Publikums abzulesen ist. Die Sprechwissenschaft und Phonetik zeigt, dass (um im Bild der Überschrift zu bleiben) die Blume „scientia orationis“ nicht nur 
außergewöhnlich und schützenswert ist. Ihre Vorteile liegen darin, an zahlreiche andere Wissenschaftsdisziplinen anknüpfen zu können und sich als Fach zu präsentieren, das in vielen Berufsfeldern Anwendung findet.

Der Studiengang „Sprechwissenschaft und Phonetik“ etablierte sich Anfang der 90er Jahre an der FSU in Jena als grundständiger Studiengang und wird seit der Bologna-Reform als Ergänzungsfach mit Bachelorabschluss angeboten. Das interdisziplinäre Fach vermittelt in anwendungsorientierter Form die Grundlagen zum selbstständigen wissenschaftlichen Arbeiten. Die Schwerpunkte in Lehre und Forschung liegen in experimenteller Phonetik und der individuellen Weiterentwicklung stimmlicher und rhetorischer Fähigkeiten. 



\title{
Sprechwissenschaftliche Therapie- und Beratungsangebote an der Universität Halle: Von den Anfängen bis in die Gegenwart
}

Zusammenfassung: Seit Beginn ihres Bestehens hat die Sprechwissenschaft durch den Transfer ihrer Expertise in die Gesellschaft Menschen zu Fragen der mündlichen Kommunikation und Sprechen beraten und therapiert. Im Beitrag wird ein historischer Abriss über die Entwicklung dieser Therapie- und Beratungsangebote bis in die Gegenwart hinein gegeben. Mit der 2019 eröffneten BESS (Beratungsstelle Sprechwissenschaft) wird die traditions- und erfolgreiche Geschichte der sprechwissenschaftlichen Beratung fortgeschrieben. Ganz im Sinne einer anwendungsorientierten Fachdisziplin werden dort stattfindende Beratungen in Lehr- und Forschungskontexte eingebunden.

Stichworte: Sprechwissenschaft Halle, Therapie, Beratung, Tradition, Entwicklung, Transfer

\begin{abstract}
Since the beginning, speech science has advised and treated people on questions of oral communication and speaking through the transfer of its expertise to society. The article provides a historical outline of the development of these treatment and counseling offers up to the present day. With the BESS (Speech Science Counseling Center) opened in 2019, the traditional and successful history of speech science advice will be continued. In keeping with an application-oriented specialist discipline, the speech science counseling is integrated into teaching and research contexts.
\end{abstract}

Keywords: Speech science in Halle, therapy, counseling, history, development, transfer 
Am 11. November 2019 wurde im Rahmen der Kleine Fächer-Wochen Sprechwissenschaft (Internetquelle 1) die sprechwissenschaftliche Beratungsstelle „BEratungsStelle Sprechwissenschaft (BESS)“ an der Abteilung Sprechwissenschaft und Phonetik der Martin-Luther-Universität Halle-Wittenberg (MLU) feierlich eröffnet und mit einer wissenschaftlichen Tagung fachlich begleitet (Internetquelle 2).

Im vorliegenden Beitrag werden die Ziele und Aufgaben der Beratungsstelle beschrieben. In Anbetracht der Geschichte der sprechwissenschaftlichen Beratung und Therapie an der MLU gilt es zunächst jedoch eine wichtige Vorläufereinrichtung vorzustellen, die „Sonderabteilung für Stimm- und Sprachstörungen“.

\section{Zur Geschichte der sprechwissenschaftlichen Beratung in Halle - „Die Sonderabteilung für Stimm- und Sprachstörungen“}

Bei der Idee zur und Planung der BESS und ihrer Aufgaben wurde eine historische und inhaltliche Betrachtung ihrer Vorgängereinrichtung, der Sonderabteilung für Stimm- und Sprachstörungen, als aufschlussreich und grundlegend für das Verständnis von einer spezifisch sprechwissenschaftlichen Beratung empfunden.

\section{$1.1 \quad$ Gründung der Sonderabteilung}

1935 berichtet Richard Wittsack (1919 bis 1952 Nachfolger Ewald Geißlers und 1929 Begründer der Abteilung für Sprechkunde am Seminar für deutsche Philologie der MLU) über die Einrichtung einer Sonderabteilung für Stimm- und Sprachstörungen. Sie war aus der Beratungsstelle für Sprechhemmungen, Sprechkrankheiten hervorgegangen (Wittsack, 1935, S. 10). Zudem bestand schon damals eine ,Beratungsstelle für Sprachgehemmte (Orthmann, 1956, S. 359). 1938 konnte er die Abteilung für Sprechkunde in das deutschlandweit in seiner Art erste Institut für Sprechkunde umwandeln. Ein Jahr zuvor hatte er zudem von Otto Brehmer die Betreuung der Phonetischen Sammlung übernommen. 
1947 erfolgte dann die Zusammenlegung aller dieser Bereiche zum Institut für Sprechkunde und Phonetische Sammlung/Sonderabteilung für Stimm- und Sprachstörungen. „Mit dieser aufwändigen Bezeichnung wurden exakt die Leitlinien fixiert, die die Arbeit des Institutes in den folgenden Jahrzehnten bestimmten" (Hirschfeld/Stock, 2014, S. 5). Wittsacks klinisch-therapeutischen Erfahrungen, die er unter anderem bei Flatau, Katzenstein und Herrman Gutzmann sen. sowie durch die Arbeit mit in den beiden Weltkriegen versehrten Soldaten gesammelt hatte, qualifizierten ihn für diese therapeutische Arbeit.

Nach dem Tod von Wittsack 1952 führte Hans Krech als dessen Nachfolger die Arbeit der Sonderabteilung für Stimm- und Sprachstörungen fort. Unter seiner Leitung fanden die Sprechstunden regelmäßig donnerstags im Gebäude des Institutes am Markt am Ende der Leipziger Straße statt, seit 1952 für viele Jahrzehnte Heimstadt der halleschen Sprechwissenschaft. Krech und alle anderen Lehrkräfte waren zwar vorrangig mit der Ausbildung der Fachstudierenden befasst. Sie wurden aber auch in hohem Maße mit der (damals sog.) „Übungsbehandlung von Menschen mit Stimm- oder Sprachstörungen“ in der Sonderabteilung beauftragt. Dabei handelte es sich anfangs sowohl um Hilfesuchende aus dem „öffentlichen Sektor“, „da es in den 50er Jahren noch keine flächendeckende Versorgung mit entsprechenden Therapieeinrichtungen gab" (Hirschfeld/Stock, 2014, S. 7) und um Mitarbeiter als auch zunehmend Studierende der Universität, insbesondere des Lehramts. Neben Menschen mit Sprach-, Sprech- oder Stimmproblemen suchten auch Schauspieler*innen, Sänger*innen, Rundfunksprecher*innen und Rezitatoren*innen in der Sonderabteilung Hilfe.

Alle Mitarbeiter ${ }^{\star}$ innen waren mit einer bestimmten Stundenanzahl und zwei Assistenten ständig in der Sonderabteilung beschäftigt (Orthmann, 1956, S. 363). Die Studierenden der Sprechwissenschaft konnten bei diesen Beratungen und Therapien zunächst hospitieren. In höheren Semestern führten sie dann die Therapien unter Anleitung auch selbst durch. 


\subsection{Frühzeitige Verbindung von sprechwissenschaftlicher Praxis, Forschung und Lehre}

So verband die Sprechwissenschaft bereits in dieser Zeit Praxis, Lehre und Forschung sehr eng miteinander, denn Krech baute auch die therapeutische Forschungs- und Publikationstätigkeit weiter aus. Er lenkte den größten Teil der verfügbaren Forschungsaktivitäten auf das Gebiet der Stimm- und Sprachstörungen. Es wurden von ihm und seinen Mitarbeiter*innen Therapiemethoden entwickelt und auch evaluiert. Exemplarisch seien hier bekannte Studien benannt, welche in dieser Zeit entstanden: Hans Krechs Habilitationsschrift 1954 „Die Behandlung gestörter S-Laute“ und „Sprechkundliche Beiträge zur Therapie der Sigmatismen“ aus dem Jahr 1955. Insbesondere letzteres war ein sehr wichtiges Buch nicht nur für die Studierenden, sondern auch für die therapeutische Arbeit in der Sonderabteilung. Noch wesentlich bedeutsamer für das sprechwissenschaftliche Paradigma der Behandlung von Menschen mit Stimmstörungen war „Die kombiniert psychologische Übungstherapie“, die KPÜ (Sammlung aller therapeutischen Publikationen von Hans Krech, 2011, HSSP-Bände 36-38).

Ein weiteres wichtiges Werk entstand durch Werner Orthmann, der ab 1952 als Assistent am Institut für Sprechkunde in Halle tätig war. Er war vorwiegend in der Übungsbehandlung von Menschen mit Sprach- und Stimmstörungen der am Institut bestehenden „Sonderabteilung für Stimm- und Sprachstörungen" tätig. So demonstrierte er an mehreren von ihm erfolgreich behandelten Menschen mit hyperkinetischen Stimmstörungen die Wirkung der Kaumethode von Emil Fröschels. Orthmann verfasste 1955 dazu seine Dissertation „Die Kaumethode und ihre Anwendung bei hyperkinetischen Stimmstörungen“. Die daraus resultierende Publikation war ein weiterer Beleg für die intensive Behandlungs- und Forschungstätigkeit am halleschen Institut. Orthmann ging später übrigens in die Bundesrepublik und wurde Professor für Sprachheilkunde an der FH Reutlingen. Er gilt heute als einer der Väter der Sprachheilpädagogik.

Zusammenfassend darf man die an Wittsacks Arbeiten anknüpfende und eigene neue Akzente setzende intensive praktische sowie Lehr- und Forschungstätigkeit Hans Krechs zu verschiedensten therapeutischen Themen als die we- 
sentliche fachwissenschaftliche Grundlage für die Entwicklung der sprechwissenschaftlichen Teildisziplin Klinische Sprechwissenschaft bezeichnen.

Eduard Kurka, der das Institut nach Hans Krechs Tod leitete, führte diesen therapeutischen Schwerpunkt des sprechwissenschaftlichen Faches fort. So widmete er seine Dissertation der Entstehung von Stimmstörungen, speziell der durch maschinelle Schreibarbeit. Ein weiterer seiner Forschungsschwerpunkte in dieser Zeit betraf das Stottern.

\subsection{Strukturelle und interdisziplinäre Anknüpfung der Sonderabteilung}

Für die Therapieangebote gab es Vereinbarungen mit der Sozialversicherung der Stadt Halle. Außerdem gab es „grundsätzliche Verbindungen“" mit der halleschen „Universitätsklinik für Ohren-, Nasen- und Kehlkopfkrankheiten“, der „Universitäts-Zahn- und Kieferklinik“ sowie der „Psychiatrischen und Nervenklinik“ (Orthmann 1956, S. 363). Wittsack arbeitet von 1930 bis zu seinem Tod fachlich sehr eng mit Adolf Eckert-Möbius zusammen. Eckert-Möbius war seit 1923 als Assistent, ab 1928 als Professor für Hals-, Nasen-, Ohrenheilkunde und von 1928 bis 1958 als Direktor der Univ.-HNO-Klinik Halle tätig. Der beeindruckende und nicht selten auch berührende Briefwechsel der beiden Professoren sowie die ausführlichen Überweisungs- und Befundberichtsschreiben zu Patient ${ }^{\star}$ innen, die beide zusammen behandelten, zeugen von einer ausgesprochen intensiven und respektvollen Zusammenarbeit auf Augenhöhe und immer mit vollem Fokus auf eine bestmögliche Behandlung der Patient*innen. Sie geben auch beredtes Zeugnis einer Zeit vor und während der Herausbildung der Stimm- und Sprachheilkunde, noch bevor sich Phonetik, Logopädie, Pädagogik und Medizin auseinanderentwickelten.

Später fertigte die Phoniatrische Abteilung der halleschen Univ.-HNOKlinik für alle zu behandelnden Menschen mit Stimmstörungen fachliche Gutachten an. Die Univ.-Zahn- und Kieferklinik überantwortete der Sprechwissenschaft Personen zur sprachtherapeutischen Nachsorge, die wegen Lippen- und/oder Kiefer- und/oder Gaumenspalten operiert worden waren. Die neurologische und psychiatrische Univ.-Klinik begutachtete im Bedarfsfall Menschen mit zentralen Sprachstörungen. 
Angesichts der wachsenden Zahl von Patient ${ }^{\star}$ innen mit organisch bedingten Artikulationsstörungen richtete die Universitätsklinik für Zahn-, Mund- und Kieferkrankheiten 1959 eine Assistenz (alsbald wissenschaftliche Mitarbeiter*innenstelle), für phonetische Anliegen - Übungsbehandlung, Forschungsprojekte - ein. Diese Stelle wurde auf Vermittlung von Hans Krech mit Gottfried Meinhold besetzt, der von 1959 bis 1964 in dieser Klinik tätig war und neben seiner Arbeit mit Patient*innen erfolgreich eine Reihe von Forschungsprojekten durchführte (zu Sigmatismen im Zusammenhang mit kieferorthopädischen Krankheitsbildern, zur pathophonetischen Diagnostik nach operativen Eingriffen im craniofacialen Bereich, auch zur Verbesserung der artikulatorischen Qualität). Eine erste Würdigung dieser erfolgreichen Kooperation zwischen dem Institut für Sprechkunde und der Klinik war die Verleihung des Jahrespreises der Deutschen Gesellschaft Zahn-, Mund- und Kieferheilkunde für die „beste in unseren Organen veröffentliche wissenschaftliche Arbeit" an den Kieferorthopäden Gerhard Grimm und die Sprechwissenschaftler*innen Gottfried Meinhold und Jutta Suttner. Als Meinhold 1964 in Jena eine Lektorenstelle an dem verwaisten Institut für Sprechwissenschaft antrat, setzte er in Jena die enge Zusammenarbeit mit der Medizin, sowohl mit der Phoniatrie als auch, vor allem seit 1982, mit der dortigen Universitätsklinik für Plastische Chirurgie/ Kiefer- und Gesichtschirurgie fort.

Es bestanden zu allen Universitätskliniken in der DDR sehr enge fachliche und kollegiale Beziehungen, auch weil dort viele Absolvent ${ }^{\star}$ innen der halleschen Sprechwissenschaft als Fachsprechwissenschaftler*innen der Medizin bzw. später als Klinische Sprechwissenschaftler*innen therapeutisch und wissenschaftlich tätig waren. Eine der engsten Beziehungen bestand zur Universitäts-HNO-Klinik Halle, insbesondere zu ihrer phoniatrischen Abteilung. Eine wichtige Person in diesem Zusammenhang war Wolfgang Pfau, HNOFacharzt, Physiologe und ausgebildeter Sänger. Pfau gehörte zum Schülerkreis von Richard Wittsack. Er hatte vor dem Medizinstudium bei ihm eine Ausbildung absolviert und war eng mit dem Institut für Sprechkunde und Hans Krech verbunden. Zusammen mit seiner Frau, der Diplom-Sprechwissenschaftlerin Eva-Maria Pfau, baute er 1954 eine phoniatrische Abteilung an der Univ.-HNOKlinik Halle auf. Auch die Tätigkeit einer weiteren Dipl.-Sprechwissenschaftlerin, Ruth Albrecht, spätere Ackermann und Schmidt, in Zusammenarbeit 
mit dem langjährigen Professor und Direktor der Univ.-HNO-Klinik Halle, Harry Jacobi, verstärkten die frühen fachlichen Verbindungen zum Institut für Sprechwissenschaft. Mit seinen ärztlichen Mitarbeiter*innen besuchte Pfau Lehrveranstaltungen Hans Krechs, später auch bei Eduard Kurka und Jutta Suttner. Suttner war viele Jahrzehnte am Institut im Bereich der später sog. Klinischen Sprechwissenschaft lehrend und forschend tätig. 1971 hat sie - basierend auf der mit Meinhold begonnenen engen Zusammenarbeit mit der Zahn-, Mund- und Kieferklinik ihre Dissertation „Postoperative Sprecherziehung bei Gaumenspaltenträgern und ihre Ergebnisse“ eingereicht. 1982 habilitierte sie zu „Subjektiven Bewertungen von Stimmen mit unterschiedlichen Heiserkeitsgraden“, eine wichtige Vorarbeit zur RBH-Klassifikation nach Nawka und Anders. Es wurden also nicht nur gemeinsam Patient ${ }^{\star}$ innen betreut. Auch in der Lehre und der Forschung ging man mit der Phoniatrie sehr erfolgreich gemeinsame Wege.

Die Zusammenarbeit mit den Kliniken war also überaus intensiv und öffentlichkeitswirksam, zu beiderseitigem Vorteil erfolgreich und durch die gemeinsamen Fachpublikationen auch von zunehmend wissenschaftlicher Bedeutung. Vor allem aber gab es einen hohen Behandlungsbedarf. So entwickelte sich das hallesche Institut bald zu einem wichtigen Therapiezentrum der DDR (Hirschfeld/Stock, 2014).

\subsection{Thematische Neuorientierung durch die therapeutische und beratende Tätigkeit}

Hans Krech verschaffte dem Institut in der philosophischen Fakultät mit diesem großen Engagement ein hohes Ansehen. Nachteil: Die Therapie war überproportionaler Arbeitsgegenstand für alle Mitarbeiter*innen. Das änderte sich 1958 mit dem Weggang von Irmgard Weithase nach München. Hans Krech wurde vom zuständigen Staatssekretariat angeboten, das Projekt zur Aussprachekodifizierung zu übernehmen. Krech übernahm und konzentrierte sich von da an stärker auf die Phonetik und Orthoepie. Die Therapie wurde quantitativ zurückgefahren. Nur Jutta Suttner therapierte noch zu 100\%. Alle anderen Mitarbeiter*innen blieben unter $50 \%$ und konzentrierten sich mehr auf sprechwissenschaftliche Lehre für die Lehramtsstudierenden. Die therapeutische Arbeit 
für den „öffentlichen Sektor“ wurde nach und nach eingestellt. Auf diesem Stand ist es dann aber auch nach Krechs Tod 1961 sehr lange geblieben.

\subsection{Sprechwissenschaftliches Therapie- und Beratungsangebot nach der Wende}

Die Therapie- bzw. Beratungstätigkeit änderte sich nach der Wende erneut. Durch die Umstellung der universitären Lehre auf das westdeutsche Hochschulsystem fielen die bis dato in der DDR obligatorischen Stimmeignungsuntersuchungen und die obligatorische Stimmbildung/Sprecherziehung für Lehramtsstudierende weg. So brauchten die Lehramtsanwärter*innen auch kein phoniatrisches Gutachten mehr und ihre stimmlich-sprecherischen Auffälligkeiten blieben und bleiben zumindest im Studium oftmals unentdeckt und unbehandelt. Daher war der Therapiebedarf während ihres Studiums auch „offenbar" nicht mehr so hoch. Nach und nach eröffneten Klinische Sprechwissenschaftler*innen und später auch Logopäd*innen sprachtherapeutische Praxen. Außerdem gab es in den Nachwendejahren eine Kündigungswelle an der Universität, die auch nicht am Institut spurlos vorbei ging. Somit waren die Kapazitäten für ein solches Angebot nicht mehr vorhanden.

Dennoch wurde das Therapie- und Beratungsangebot weiter aufrechterhalten und es wurde auch gern wahrgenommen. Neben interessierten und gut informierten Lehramtsanwärter*innen kamen auch immer noch Erwachsene und Kinder in die Therapie und zur Beratung. Die letzten am Institut auch therapeutisch tätigen Klinischen Sprechwissenschaftlerinnen waren neben Jutta Suttner vor allem Gerda Falgowski, Dr. Leila Greifenhahn-Kell und Annette Leutloff. Mit ihrem Ausscheiden 2001 bzw. 2006 und unter damals sehr prekären Stellenverhältnissen verschwanden die Therapie- und Beratungsangebote der Sprechwissenschaft allmählich und für längere Zeit.

\section{BESS: BEratungsStelle Sprechwissenschaft heute}

Auch wenn sich die hallesche Sprechwissenschaft in den letzten beiden Jahrzehnten auf teilweise völlig neue Aufgaben fokussierte, so geriet das Thema 
„Beratung" und die therapeutische Unterstützung durch klinisch sprechwissenschaftliche Expertise dennoch nie ganz in Vergessenheit bzw. bekam auch neue „Impulse“. So war das Thema im Rahmen der Spezialisierung im Masterstudium „Klinische Sprechwissenschaft“ immer relevant und von grundlegender Bedeutung.

Auf Initiative von Susanne Voigt-Zimmermann nach deren Berufung auf die W3-Professur für Sprechwissenschaft im Jahr 2017 wird nunmehr mit der Einrichtung der „BESS“ die traditions- und erfolgreiche Geschichte der sprechwissenschaftlichen Beratung und mit ihr die Fachgeschichte fortgeschrieben.

\subsection{Inhalt, Ziele und Organisation der Beratungsstelle}

Die Beratungsstelle möchte auch heutzutage Studierende, Auszubildende und Beschäftigte der Universität darin unterstützen, ihre kommunikativen Fähigkeiten in sozialen, gesellschaftlichen und vor allen Dingen auch berufsbezogenen Kontexten zu reflektieren und zu optimieren. Das kann alle Aspekte des (Miteinander-)Sprechens betreffen: beginnend bei der eigenen Stimme, den mundmotorischen und phonetisch-phonologischen Fähigkeiten bis hin zur Sprechverständlichkeit. Es können Fragen zu latent und tatsächlich dialogischen Situationen bearbeitet werden, wie zu Rede- und Vortragstätigkeit, zum Sprechen in den Medien, zu Gesprächsführung, Redevorbereitung oder rhetorische Wirksamkeit. Nicht zuletzt sind auch Sprechhemmung und Sprechangst zentrale Themen, zu denen beraten und unterstützt wird.

Die Beratungsangebote verstehen sich dabei grundsätzlich als - unter Umständen ersten - Anstoß zur Veränderung. Deshalb steht eine klienten- und lösungsorientierte Vorgehensweise im Vordergrund. Das bedeutet, dass die Themen immer mit den Klienten gemeinsam mit dem Ziel erarbeitet werden, persönliche Potentiale sichtbar zu machen. Je nach Beratungsbedarf kommen nach einer Auftragsklärung dann viele unterschiedliche Methoden zum Einsatz, wie zum Beispiel eine videobasierte Analyse, konkrete Empfehlungen oder auch Übungen. Stellt sich heraus, dass die Fragestellung komplexer ist, so werden entsprechende fachkundige Institutionen vor Ort einbezogen. Die Beratungstermine werden individuell vereinbart. Es können Einzelcoachings wie auch Gruppengespräche wahrgenommen werden. 
Ein weiteres sehr wichtiges Ziel der BESS ist es, die Beratungen mit der sprechwissenschaftlichen Lehre und Forschung zu vernetzen, denn ein wichtiger Aspekt des Studiums ist die Vermittlung von Beratungskompetenzen. Die Beratungsstelle ist deshalb ein für uns sehr wichtiges Element der engen Verzahnung von sprechwissenschaftlicher Praxis und Lehre. Deswegen wurden die Räumlichkeiten der BESS baulich so konzipiert, dass Beratungssituationen durch eine semitransparente bidirektionale Spiegelscheibe und akustische Übertragungsanlage live beobachtet, aufgezeichnet, ausgewertet und ebenso live gecoacht werden können. Das ermöglicht es uns, unsere Lehre nicht nur praxisnah zu gestalten, sondern auch wissenschaftlich zu evaluieren.

Die Beratungsstelle befindet sich in der Abteilung Sprechwissenschaft und Phonetik des Institutes für Musik, Medien- und Sprechwissenschaften in der Emil-Abderhaldenstraße 26-27. Die Beratungen werden vorrangig von Mitarbeiter*innen der Sprechwissenschaft durchgeführt. Auch höhersemestrige Studierende können unter Supervision beraten, wenn sie die nötige fachliche Eignung erworben haben und das Einverständnis durch die zu Beratenden gegeben ist. Ansprechpartnerin zur ersten Kontaktaufnahme ist Dr. Stephanie Kurtenbach. Ein Beratungswunsch kann per E-Mail an folgende Adresse gerichtet werden: sprechberatung@sprechwiss.uni-halle.de.

\subsection{Eröffnung der BESS -BEratungsStelleSprechwissenschaft mit der Tagung: „Beratung in Forschung, Lehre und Praxis“}

Mithilfe der ideellen und finanziellen Unterstützung der Hochschulrektorenkonferenz und des Bundesministeriums für Bildung und Forschung im Rahmen der „Kleine-Fächer-Wochen Sprechwissenschaft“ konnte die "Sprechwissenschaftliche Beratungsstelle - BESS“ mit einer Fachtagung und anschließender Podiumsdiskussion feierlich eröffnet werden. Diese fand am 11. November 2019 im Seminar- und Tagungsraum der Sprechwissenschaft am Steintorcampus statt.

Die Sprecherin der Abteilung, Prof. Susanne Voigt-Zimmermann, gab als Initiatorin der neu eingerichteten Beratungsstelle den Auftakt zur Tagung. Hierbei dankte sie insbesondere dem Rektorat für die Begleitung bei der Antragstellung. Außerdem nutzte sie die Gelegenheit, ausführlich darauf hinzu- 
weisen, wie intensiv das Rektorat wie auch das Dekanat der philosophischen Fakultät II die „Kleine -Fächer-Wochen Sprechwissenschaft" unterstützt haben. So hat die Universität beispielsweise die baulichen Maßnahmen zur Einrichtung der Beratungsstelle finanziert. Dass die Universität die Beratungsstelle als besonders wichtig erachtet, drückte sich in der Teilnahme sowohl des Prorektors für Studium und Lehre, Prof. Wolf Zimmermann aus, als auch der Leiterin der Stabstelle des Rektorats, Frau Katrin Rehschuh, sowie der Pressesprecherin, Frau Manuela Bank-Zillmann. Abschließend hob Voigt-Zimmermann hervor, welch großen Stolz und Freude die Sprechwissenschaft erfüllen, die Tradition der von Wittsack vor fast 100 Jahren gegründeten Sonderabteilung für Sprachund Stimmstörungen mit der BESS wieder aufzunehmen und als Erfolgsgeschichte fortzuführen. Damit sind der Wunsch und die Hoffnung verbunden, als integrierende Eirichtung aller im Bereich Beratung und Therapie tätigen Einrichtungen der Universität wahrgenommen zu werden.

Es folgten die Grußworte: Prof. Dr. Wolf Zimmermann, Prorektor für Forschung und Lehre der Martin-Luther-Universität, gratulierte der Abteilung zu diesem für die Universität sehr bereichernden Angebot und drückte seine Freude darüber aus, das Fach Sprechwissenschaft im Zuge der 2019 erfolgten Akkreditierung in seiner großen Bandbreite kennengelernt zu haben. Dabei betonte er auch die besondere Bedeutung des Studienganges Sprechwissenschaft bei der Vermittlung von spezifisch sprechwissenschaftlicher Beratungskompetenz. Er wünschte der Abteilung mit der Beratungsstelle viel Erfolg und eine intensive Beratungstätigkeit. Im Anschluss sprach die Vorsitzende des Deutschen Bundesverbandes Klinischer Sprechwissenschaftler (DBKS e. V.), Frau Gabriele Finkbeiner. Sie wies vor allem auf die Bedeutsamkeit des Praxisbezuges der Sprechwissenschaft in all ihren Teildisziplinen hin. Sie gratulierte dem Fach dafür, mit der Eröffnung der Beratungsstelle Sprechwissenschaft auch für die Studierenden eine Möglichkeit zu schaffen, das in der Theorie Gelernte praktisch reflektieren und erproben zu können.

Im Anschluss begann der fachliche Teil der Veranstaltung. Stephanie Kurtenbach informierte mit einem historischen Exkurs über die Bedeutsamkeit der Sprechwissenschaft als Beratungsinstitution seit Bestehen des Faches (vgl. Kapitel 1). Michaela Kupietz sprach über Inhalt, Ziele und Organisation der Beratungsstelle (vgl. Kapitel 2.1). 
Eine ganz praktische Vorstellung der Beratungstätigkeit konnte mit einem Imagefilm, der durch das Hochschulmarketing der Martin-Luther-Universität gedreht wurde, präsentiert werden (\#unihalle Sprechwissenschaftliche Beratungsstelle | Uni Halle, Internetquelle 3). Dieser wurde in den Beratungsräumen der Sprechwissenschaft gedreht und dokumentiert die Beratungstätigkeiten wie auch deren Einbindung in Forschung und Lehre.

Es folgten im Weiteren die Fachvorträge der eingeladenen Beratungsexpert*innen. Den Auftakt machte eine ausgewiesene Expertin der systemischen Beratung, Frau Dr. med. Ulrike Jänicke aus Leipzig. Zum Thema „Fragen statt Sagen - Die Bedeutung von Sprache in der systemischen Beratung" berichtet sie eindrucksvoll über ihre Erfahrungen in Beratungs- und Coachingprozessen. Ihre zutiefst wertschätzende Haltung gegenüber Klient ${ }^{\star}$ innen gab diesem fachlich hochkarätigen Vortrag eine sehr persönliche Note. Es folgte ein ganz praktischer Beitrag: Christin Alexandrow und Matthias Winter stellten ihren Coachingansatz „Comisch“ (siehe dazu den Artikel von Matthias Winter in diesem Band) vor und führten diesen per Live-Beratung mit einer Tagungsteilnehmerin durch. Nicht nur war der Ansatz für alle Teilnehmer*innen, sondern auch das Wiedererkennen der von Jänicke beschriebenen systemischen Fragetechniken in dieser Live-Beratung außerordentlich spannend. Anschließend sprach Frau Dr. Ina Pick von der Universität Basel zum Thema „Beraten in Interaktion. Zur gesprächslinguistischen Bestimmung des Beratens“. Auch dieser Vortrag war hochinteressant, denn Picks Perspektive auf das Beraten ist mehrdimensional: Als Gesprächsforscherin und Beraterin verbindet sie ihre praktische Arbeit mit dem wissenschaftlichen Evaluieren von Beratungssituationen.

Das Tagungsende bildete eine anregende Podiumsdiskussion mit anderen Beratungsstellen der halleschen Universität. Unserer Einladung zu diesem Austausch folgten Judith Portius vom Team der Personalentwicklung, Tino Schlögl vom Career Center und Annelie Breitfeld von der Allgemeinen Studienberatung. Gemeinsam diskutierten unsere Gäste mit den Fachvertreterinnen der Sprechwissenschaft Prof. Susanne Voigt-Zimmermann, Dr. Stephanie Kurtenbach und Michaela Kupietz. Judith Portius stellte die neue Ausrichtung der Personalpolitik der Universität vor, der es vor allem darum geht, eine systematische und bedarfsgerechte Personalentwicklung voranzutreiben. Tino Schlögl berichtete von seiner Beratungstätigkeit für Studierende 
und Mitarbeitende, die Unterstützung bei der Karriereplanung benötigen. Annelie Breitfeld reflektierte ihr breites Bratungsspektrum, das auch gesundheitliche Fragen wie zum Beispiel psychisch belastende Situationen während der Studienzeit beinhaltet. Es war allgemeiner Konsens der Podiumsgäste, dass der Beratungsbedarf all dieser Angebote sehr hoch ist. Die Podiumsdiskussion endete mit einem Austausch darüber, wie die verschiedenen Beratungsangebote noch intensiver miteinander vernetzt werden können, um voneinander $\mathrm{zu}$ profitieren. Gemeinsame Weiterbildungen zu fachübergreifenden Fragestellungen im Zusammenhang mit Beratung, datenschutzrechtliche Fragen wie auch Möglichkeiten zu einem regelmäßigen Austausch der Beratungsstellen wurden diskutiert. Diese Podiumsdiskussion bildete einen gelungenen Abschluss einer interessanten, abwechslungsreichen und gesprächsintensiven Tagung.

So teilen wir auch das positive Feedback der über hundert Teilnehmenden und Referent ${ }^{\star}$ innen: Interessante Perspektiven, Erfahrungen und wissenschaftliche Erkenntnisse zu allgemeinen und spezifischen Aspekten des Beratens konnten zusammengebracht, diskutiert und erlebt werden. Ein toller Start für die Beratungsstelle Sprechwissenschaft!

\subsection{Erste Erfahrungen mit der Beratungsstelle Sprechwissenschaft}

Seit der Eröffnung der Beratungsstelle Sprechwissenschaft im November 2019 werden regelmäßig Anfragen über die Kontaktmailadresse gestellt. Es waren sowohl Universitätsbeschäftigte als auch Studierende, die das Angebot bisher nutzten. Die Anliegen sind ganz unterschiedlicher Art, berührten bisher jedoch immer Themen der mündlichen Kommunikation. Mehrere Nichtmuttersprachler*innen konnten zu Fragen der Aussprache beraten und auch trainiert werden. Einige Anfragen bezogen sich auf stimmliche Herausforderungen im beruflichen Kontext wie das Sprechen vor großen Gruppen oder das intensive Telefonieren in beratenden Funktionen. Mehrfach gab es auch den Wunsch, Möglichkeiten kennenzulernen, um die eigene Unsicherheit in Gesprächs- oder Vortragssituationen abzubauen. Das Thema Sprechtempo war ebenso ein Anliegen mehrerer Anfragenden: Wie kann das Sprechtempo in Gesprächs- und Vortragssituationen im Sinne einer besseren Verständlichkeit kontrolliert werden? Viele Beratungsanliegen konnten in ein bis drei Terminen 
erfolgreich bearbeitet werden. Bei ungefähr einem Drittel der Anfragen stellte sich heraus, dass die Thematik komplexer ist, als in einer Beratung zu lösen. Zum Beispiel gab es mehrere Personen, deren stimmliche oder artikulatorische bzw. mundmotorische Beschwerden so beträchtlich erschienen, dass eine fachärztliche Abklärung veranlasst wurde. Die Beratungsangebote sind bisher von allen dankbar angenommen worden. Das erfreut die Mitarbeiter*innen der Beratungsstelle aus mehreren Gründen: Es untermauert die Relevanz und den Bedarf dieser Einrichtung und die Rückmeldung, dass eine Beratung sehr hilfreich ist, bestärkt die Beratenden außerdem in ihrer Funktion. Durch die Corona-Pandemie änderte sich die Beratungstätigkeit im März 2020 plötzlich. Aufgrund der während der Pandemie geltenden Hygienevorschriften der Universität wurden die Beratungen per Video- oder Telefonkonferenz durchgeführt. Dieses Angebot wurde ebenso intensiv wahrgenommen. Durch die aktuellen Lockerungen der Hygienevorschriften können die Beratungen nun wieder in Präsenz stattfinden. Nach den unerwartet positiven Erfahrungen im Sommersemester werden jedoch weiterhin auch digitale Beratungen angeboten.

\subsection{Weitere Vorhaben}

Neben der beratenden Tätigkeit sind weitere Vorhaben in Planung oder werden bereits umgesetzt: Dokumentations- und Evaluationsmaterialien werden entwickelt und erprobt, um Zielgruppen, Beratungsinhalte und Effekte systematisch zu erheben. Diese Aufbereitung bildet für uns ein wichtiges Fundament zur Entwicklung von Forschungsfragen und Beratungskonzepten. Eine Internetseite ist in Planung, um das Angebot noch zugänglicher zu machen. Weitere Integrationsmöglichkeiten in die Lehre werden mit den Kolleg*innen der Sprechwissenschaft entwickelt. Für die Umsetzung dieser Ziele war die Installation eines multiperspektivischen Video- und Audiosystems von großer Bedeutung.

Dieser technische Fortschritt stellt eine wesentliche Bereicherung für die sprechwissenschaftliche Beratung dar und unterstützt außerdem die Ziele der Beratungsstelle nachhaltig Lehre, Forschung und Praxis miteinander zu vernetzen. Und doch war ist und bleibt das Beraten in der BESS immer ein Gespräch unter Menschen über das Sprechen von und mit Menschen. 


\section{Danksagung}

Unser herzlicher Dank geht an unsere ehemaligen Lehrer*innen und späteren Kolleg^innen Prof. Dr. Eberhard Stock, Marle Noglik, Gerda Falgowski und Dr. Leila Greifenhahn-Kell, die uns mit ihren Erinnerungen geholfen haben, die Anfänge und die Geschichte der sprechwissenschaftlichen Beratung und Therapie an der MLU nachzuzeichnen. Alle waren bis zu ihrem Eintritt in die Rente bzw. im Falle Stocks bis zur Emeritierung am (damaligen) Institut für Sprechwissenschaft und Phonetik tätig. Eberhard Stock war von 1967 bis 1976 und von 1981 bis 1993 zudem Leiter des Institutes. Marle Noglik und Gerda Falgowski waren als Mitarbeiterinnen in der Lehre zur Sprecherziehung und Stimmbildung tätig, einerseits für die eigenen Fachstudent ${ }^{*}$ innen und Marle Noglik auch für Studierende des Lehramts. Dr. Greifhahn-Kell kam 1987 ans Institut. Neben ihrer Vorlesungs- und Seminartätigkeit unterstützte sie die therapeutische Lehre und Arbeit durch ihre ausgewiesene klinische Erfahrung, die sie als klinisch tätige Sprechwissenschaftlerin zuvor an der HNO-Abteilung des Klinikums Dessau gesammelt hatte. Prof. Dr. Gottfried Meinhold danken wir für die Erinnerungen an die wichtige Zusammenarbeit zwischen der halleschen Sprechwissenschaft und der „Zahnklinik“. Ein weiterer Dank gebührt Prof. Dr. Stefan Plontke für die historischen Details die hallesche Univ.-HNOKlinik betreffend, deren Direktor er jetzt ist. 


\section{Literatur}

Hirschfeld, Ursula/Stock, Eberhard (2014): Erinnerungen an Hans Krech. In. Sprechen 58, S. 4-15.

Orthmann, Werner (1956): Sprechkundliche Behandlung funktioneller Stimmstörungen: Anwendung der „Kaumethode“ (Fröschels, Chewing approach) für hyperkinetische Stimmstörungen. Halle (Saale): Marhold. (Beiträge zum Sonderschulwesen Bd. 11)

Orthmann, Werner (1956): Fünfzig Jahre sprechkundliche Arbeit in Halle. In: Krech, H. (Hg.): Festschrift zum 50-jährigen Bestehen der sprechkundlichen Arbeit an der Martin-Luther-Universität Halle-Wittenberg. Halle, S. 356-364. (Wiss. Z. Univ. Halle, GSR V)

Stock, Eberhard (2019): Fachgeschichtliche Notizen: Zur Entwicklung der halleschen Sprechwissenschaft zwischen 1945 und 1990. Berlin: Frank \& Timme. (= SSP Bd. 21)

Weithase, Irmgard (1952): Richard Wittsack zum Gedächtnis. Zeitschrift für Phonetik und allgemeine Sprachwissenschaft 5/6, S. 373-378.

Wittsack, Richard (1935): Die Abteilung für Sprechkunde an der Martin-LutherUniversität Halle-Wittenberg. Halle/Saale.

\section{Internetquellen}

Internetquelle 1: https://kfw.sprechwiss.uni-halle.de/ (Letzter Zugriff am 24.2.2021) Internetquelle 2: https://kfw.sprechwiss.uni-halle.de/eroeffnung-dersprechwissenschaftlichen-beratungsstelle/) (Letzter Zugriff am 24.2.2021)

Internetquelle 3: Imagefilm BESS https://www.youtube.com/watch?app= desktop\&v=d_teNR6ZH3c\&feature=emb_title (Letzter Zugriff am 24.2.2021) 
Stephanie Kurtenbach / Steffi Schwarzack / Dirk Meyer

und Susanne Voigt-Zimmermann, Halle (SaAle)

\title{
Sprechwissenschaftliche Beratung - eine Haltungsfrage?
}

Zusammenfassung: Menschen zu Fragen der mündlichen Kommunikation und Sprechen zu beraten, ist immanenter Gegenstand der sprechwissenschaftlichen Lehre und Forschung. Was aber zeichnet eine sprechwissenschaftliche Beratung aus? Darüber führen vier Sprechwissenschaftler*innen ein Gespräch, aus dem deutlich wird, dass Beratung zwei ganz grundlegenden Perspektiven folgt. Eine konstruktivistische Perspektive prägt die Haltung der Beratenden in Form einer Begleitung: Klient*innen werden als Expert*innen für ihre Lösungen angesehen. Eine fachwissenschaftliche Perspektive ermöglicht es, konkrete Techniken zur Optimierung kommunikativer Fähigkeiten zu vermitteln.

Stichworte: Sprechwissenschaft, Beratung, Anspruch, Haltung, Vermittlung

\begin{abstract}
Consulting people on questions of oral communication and speech is an inherent part of teaching and research in speech science. But what distinguishes such a consulting? Four speech scientists conduct a conversation about this. It becomes clear that counseling follows two very fundamental perspectives. A constructivist perspective shapes the attitude of the counselors in the form of accompaniment: clients are seen as experts in their solutions. A professional perspective enables clients to learn specific techniques for optimizing their speaking behavior.
\end{abstract}

Keywords: Speech Science, counseling, demand, attitude, teaching

Anlässlich der Eröffnung der Sprechwissenschaftlichen Beratungsstelle „BESS“ am 11.11.2019 (Internetquelle 1) fragten wir uns, wie das Verhältnis von Beratung und Sprechwissenschaft derzeit ist. Die Fragen an Stephanie Kurtenbach, 
Dirk Meyer und Steffi Schwarzack stellte am 19. Juni 2020 Susanne VoigtZimmermann, Sprecherin der Abteilung Sprechwissenschaft und Phonetik. Stephanie Kurtenbach ist aktiv in die Sprechwissenschaftliche Beratung der BESS eingebunden. Dirk Meyer unterrichtet im Bereich Rhetorische Kommunikation. Steffi Schwarzack ist als selbstständige Sprechwissenschaftlerin beratend im Unternehmenskontext tätig und aktuell Lehrkraft für besondere Aufgaben.

\section{Interview}

Susanne: Wie seht ihr das Verhältnis von Sprechwissenschaft und Beratung heute?

Stephanie: Mir fallen da die ganzen unterschiedlichen sprechwissenschaftlichen Beratungskontexte ein. Am vertrautesten ist mir die Therapie. Da spielt die Beratung tatsächlich eine sehr wichtige Rolle, da es in sämtlichen Therapiephasen beratende Situationen gibt. Das beginnt gleich am Anfang mit der Auftragsklärung (Was will der/die Patient*in, was wollen die Eltern für das Kind). Dann geht es mit dem Anamnesegespräch weiter, in dem erst einmal Informationen über Fragen eingeholt werden, doch teilweise gibt es auch beratende Phasen und zwar immer dann, wenn die Patient ${ }^{\star}$ innen Fragen haben. Im Stecken von Therapiezielen habe ich als Therapeutin auch eine beratende Funktion, indem bspw. verschiedene Varianten mit den Patient*innen besprochen werden. Schon zu Beginn meiner therapeutischen Tätigkeit habe ich festgestellt, dass mir die Tools der Sprechwissenschaft, besonders aus dem rhetorischen Bereich, sehr helfen, diese Beratungssituationen zu bewerkstelligen.

Susanne: Als ich Ende der 1980er Jahre studiert habe, war das Thema Beratung eher implizit, es lief parallel in den verschiedenen Lehrveranstaltungen und sprecherzieherischen Kontexten mit. Beratungsaspekte wurden explizit bei der „Kombiniert psychologischen Übungstherapie“ (KPÜ) von Krech thematisiert. Zudem war der Fokus stark auf das sofortige Helfen(-Können) gelegt. Man sollte beispielsweise Patient*innen schnell ein Hilfsangebot machen. Da 
hat in den letzten Jahren ein Paradigmenwechsel stattgefunden. Dirk, was hat sich denn aus deiner Sicht am Herangehen in der sprechwissenschaftlichen Beratung am stärksten geändert?

Dirk: Ich halte diese Auftragsklärung auch in der Rhetorik für sehr wichtig. Ich berate auch in der Rhetorik niemanden, der mich nicht explizit um meine Meinung und um ein Feedback bittet. Und selbst wenn das passiert, halte ich mich mit Ratschlägen sehr stark zurück, weil das meiner Grundauffassung von Rhetorik entspricht. Denn ich weiß nicht, was im rhetorischen Bereich „richtig“ oder „falsch“, „gut“ oder „schlecht“ ist. Stattdessen arbeite ich mit Kategorien wie „viabel“ oder „nicht-viabel“, also funktioniert es oder funktioniert es nicht. Wenn ich in der Rhetorik jemanden beraten würde - was die Student*innen durchaus wollen - im Sinne von Tipps und Hinweisen, wie sie es "richtig“ machen müssten oder „besser“, dann laufe ich Gefahr, dass sie diese Tipps dann generalisieren und es in der nächsten Situation ganz genauso wieder machen. Genau in dieser Situation ist es aber möglicherweise nicht viabel. Dann funktioniert es nicht und dann bekommen sie auch das Feedback, dass das jetzt nicht gut war. Daraufhin sagen die Student*innen dann völlig zu Recht: „Der Meyer hat aber gesagt, so und so funktioniert es“. Deswegen halte ich mich da stark zurück und wenn überhaupt, dann berate ich nur nach einer entsprechenden Auftragsklärung. Damit ich weiß, was jemand ganz konkret von mir erwartet und möchte.

Steff: Wenn wir den Blick auf den freien Berater- und Coaching-Markt wenden, finden wir genau diese Haltung auch. Es gibt eine ausgeprägte Diskussionskultur darüber, was Beratung und Coaching je sind. Das wird mitunter ganz scharf voneinander abgetrennt. Theoretisch, weil das praktisch meiner Meinung nach nicht geht. In der Theorie ist Beratung im engen Sinne das, was offensichtlich du, Susanne, früher in der Sprechwissenschaft und der Klinikarbeit erlebt hast, also die Auffassung „Ich weiß, wie es geht“, „Ich hab die Lösung“. Das Coaching will sich davon sehr stark abtrennen. Man will den Klient*innen auf Augenhöhe begegnen. Der Coach ist lediglich für den Prozess verantwortlich, die Lösung soll Jede oder Jeder selbst finden. Die sprechwissenschaftliche Beratung verbindet beides, ein Sowohl-als-auch. 
Susanne: Ist diese Trennung von Beratung und Coaching also nur eine theoretische?

Dirk: Also nach meiner Kenntnis gibt es ja sowas wie die Berater-Szene oder die Beratung gar nicht. Es gibt da ganz unterschiedliche Schulen und unterschiedliche Ansichten. Ich überblicke schon ganz gut, dass es bspw. in den systemischen Ansätzen und im hypnosystemischen Ansatz von Gunther Schmidt überhaupt nicht darum geht, dass jemand weiß, wie es richtig ist oder wie es geht, und dem Klienten das mitteilt. Da geht es darum, dass die vor allen Dingen über entsprechende Interventionen und über Fragen usw. bei den Klienten selber Lösungs- und Veränderungsversuche zu initiieren versuchen. Und gerade die hypnosystemische Beratung geht ja grundsätzlich von der Ericksonschen Potenzialhypothese aus. Diese besagt, dass jeder Mensch alle Ressourcen in sich hat, um mit seinem Leben klar kommen zu können. Man ist nur entweder mit diesen Ressourcen assoziiert oder dissoziiert. Und wenn man nun aktuell „Probleme“ hat, sind eben bestimmte Ressourcen dissoziiert. Die sind aber dennoch da. Und es gilt dann im Prozess der systemischen Beratung zu versuchen, dissoziierte Ressourcen wieder zu assoziieren.

Susanne: Meine über zwei Jahrzehnte andauernde klinische Arbeit ist natürlich stark von ärztlicher Beratungserfahrung geprägt. Das ist viel Arbeit am Symptom und auf der Mikroebene, ganz nah an der Organstruktur. Bei funktionell bedingten oder psychogenen Störungen oder etwa psychosozialen und psychoonkologischen Fragen wird dann stärker gemeinsam mit dem Patient*innen an selbst entwickelten Ideen gearbeitet, wie das Problem in den Griff zu bekommen ist und welche Elemente der Makro- oder Mesoebenen zur Lösung des Problems herangezogen werden müssen. Man merkt auch in der Medizinerausbildung, dass sich da ganz viel tut, sich der Ton ändert, Patient*innen als Partner*innen stärker ernst genommen und mehr in die Lösungsentwicklung eingebunden werden. Aber jetzt zurück zur sprechwissenschaftlichen Beratung.

Stephanie: Eigentlich haben wir in der Therapie nur Kontexte, in denen wir sofort in ein gemeinsames Gespräch gehen. Weil wir ja letztlich immer an 
funktionalen Themen gemeinsam arbeiten. Deshalb dürfen wir in der Therapie natürlich nicht ausklammern, dass es Situationen gibt, in denen wir "Weisende“ oder „Hinweisende“ sind. Und trotzdem gilt die Matrix, dass wir alle Themen im Gespräch klären. Die sprachtherapeutische Realität ist leider oft noch anders. Diese Denke „Ich bin der Experte und weiß, wie es richtig ist“ steckt in vielen Köpfen noch ganz manifest. Weil die Therapie eben ein Stück weit zur Medizin gehört und der Gedanke der Teilhabe an der Gestaltung meines Behandlungsprozesses auch in der Medizin noch sehr jungfräulich ist. Ich glaube, da müssen wir noch viel Aufklärungsarbeit leisten.

Steff: Ich erlebe das im nicht-therapeutischen Bereich tatsächlich auch. Klient ${ }^{\star}$ innen kommen teils mit einer gottgleichen Erwartungshaltung. Auftragsklärung ist dann das Stichwort. Was will der bzw. die Kundin? Was braucht er bzw. sie? Das sind zwei verschiedene Paar Schuhe. Manche kommen z. B. mit der Erwartung ihre Verantwortung abzugeben: „Meine Stimme klingt zu leise. Kannst du da was machen?". Dass es auch um andere Aspekte gehen kann, wie „Darf ich überhaupt laut sein? Erlauben mir meine inneren Instanzen das?“, das gehört zur Auftragsklärung. Im Sinne von Aufklären. Aber das ist eben nicht immer gewollt.

Dirk: Ich glaube, dieser Wunsch ist ein zutiefst menschliches Bedürfnis. Das hängt damit zusammen, dass wir uns im Alltag - wir alle, ich auch - wünschen, dass wir immer dann, wenn ein Problem auftritt, vor allem ein Problem, das in einem komplexen, komplizierten Zusammenhang steht, jemanden haben, der in der Lage ist, dieses Problem zu trivialisieren und Lösungen dafür zu finden. Bei mir ploppt da sofort das Modell der trivialen und nicht trivialen Maschine auf (Foerster, H. v. 2009, 60ff.). Bei der trivialen Maschine erwarten wir beispielsweise wie im Auto, dass dieses, wenn wir die Zündung anmachen, anspringt und dann fährt. Und wenn das dann mal nicht anspringt, rufen wir einen Experten, der die Trivialität dieser Maschine wiederherstellt. Der Mensch ist aber nun mal keine triviale Maschine, sondern eine nicht-triviale Maschine. Und deswegen ist er auch nicht so ohne weiteres zu trivialisieren. Das wünschen sich aber Menschen und diesen Wunsch kann man auch niemandem vorwerfen. 
Steffi: Da dieser Wunsch manchen Klient*innen gar nicht bewusst ist, müssen wir als Beratende die genauen Erwartungen und Motive auch erst in Erfahrung bringen und herausfinden, in welche Richtung ich überhaupt arbeiten darf. Steve de Shazer (2019) hat da sein sehr praktikables Modell der „Kunden“, „Besucher“ und „Klagenden“ eingeführt. Nur als „Kunde“ sind Klient*innen arbeitsbereit. Und ich sollte im ersten Gespräch, aber eigentlich in jeder Stunde der Zusammenarbeit erneut, herausfinden, in welcher Rolle meine Klient*innen gerade vor mit sitzen. Wollen sie heute vielleicht einfach nur jammern?

Susanne: Klient*innen wollen dann nicht lösungsorientiert arbeiten, nur einfach mal Dampf ablassen, auch verständlich. Und dann bist du die Einzige, der man das erzählen kann.

Steffi: Es war für mich ein Lernprozess das auszuhalten. Dass ich auch für so ein Gespräch Geld nehmen darf. Also dafür, dass ich „nur“ zuhöre und nicht berate.

Susanne: Wir reden immer wieder in der Abteilung darüber, wie unsere Studiengänge modifiziert werden müssen und was da inhaltlich und methodisch mit rein muss.

Dirk: Wenn ich an mein Studium in den 80ern zurückdenke, dann bin ich sehr dankbar für viele, viele sprechwissenschaftliche Grundlagen, die ich erhalten habe und die mir auch heute noch helfen. Wenn ich auf meine heutige Arbeit blicke, dann stammt gerade in der Rhetorik vielleicht 90\% dessen, was ich heute vertrete und was ich heute mache, aber nicht mehr aus dem Studium. Das habe ich mir danach erschlossen. Verschiedene Dinge vor allem aus der Philosophie, Psychologie und Psychotherapie, haben mich sehr stark beeinflusst. Z. B. halte ich eine konstruktivistische Weltanschauung für sehr sinnvoll und wertvoll und die hat auch meine Arbeit massiv beeinfluss. Was ich noch für sehr wichtig halte ist, dass die Student ${ }^{\star}$ innen sich eine Haltung zulegen. Und dass sie mit einer bestimmten Haltung an ihre Jobs rangehen, egal in welchem Bereich der Sprechwissenschaft sie tätig sind. Mehrere Masterstuden*innen aus dem ersten Studienjahr haben mir gesagt „Wir sind ja auch alle in einer 
bestimmten Art und Weise geprägt.“ D. h. es ist uns möglicherweise gelungen, bei ihnen schon eine gewisse Haltung anzubahnen, die eben nicht darin besteht, jetzt nun alles besser und ganz genau zu wissen usw., sondern einfach respektvoll und wertschätzend mit ihrer Klientel umgehen, egal woher diese kommt oder in welchen Bereichen sie da arbeiten.

Stephanie: Ich würde gerne noch etwas aus meiner Perspektive zur Haltung sagen, die sehr aus der systemischen Berater ${ }^{*}$ innenausbildung geprägt ist. Die systemischen Grundhaltungen haben mit der Rhetorik zu tun, die Dirk gelehrt hat. Also im Sinne Helmuth Geißners, dass wir etwas zur gemeinsamen Sache machen. Wir manipulieren nicht Menschen, sondern wir kommen miteinander ins Gespräch. Das war etwas, was ich sozusagen mit dieser systemischen Beratung als ein und dasselbe erfahren habe. Die systemische Herangehensweise durchleuchtet diese Haltungen nochmal ganz genau. Dazu gehört genau die Wertschätzung, Neutralität oder Allparteilichkeit und auch Neugier. Mit Neugier kann ich meine eigenen Vorurteile über Bord werfen. Und dann lerne ich mehr Fragen zu stellen.

Susanne: Sollten wir nach solch einer Haltung bereits bei den Eignungsprüfungen schauen und kann man Haltung prüfen?

Stephanie: Meines Erachtens kann man sowas nicht prüfen. Ich würde mich eher mit den Studierenden gemeinsam auf eine Reise begeben. Also miteinander diese Haltungen reflektieren und in bestimmten Situationen und Gesprächssituationen üben. Und das machen wir ja auch in Seminaren und Projekten mit Kitas. Da geht es sehr viel um Haltung. Also klar, wir vermitteln da auch Wissen. Aber das erste, was wir mit den pädagogischen Fachkräften und Studierenden machen, ist, sie mit ihren eigenen Haltungen feinfühlig zu konfrontieren, weil nur durch eine veränderte Haltung oder durch ein Bewusstwerden darüber, wie meine Haltung zum Beispiel zu mehrsprachig aufwachsenden Kindern, zu mehrsprachigen Familien ist, können Änderungsprozesse angekurbelt werden. Meine Erfahrung ist mittlerweile, dass das gut gelingt. Deshalb würde ich nie sowas testen wollen. 
Dirk: Auch ich bin der Überzeugung, dass man Haltung nicht wirklich lehren oder prüfen kann. Sondern man kann und muss Haltung vorleben. Und in dem Moment, wo man eine bestimmte Haltung lehrt, und sie selber nicht lebt, wirkt man völlig unglaubwürdig. Es ist wahnsinnig schwer, weil wir alle permanent Gefahr laufen, in bestimmten Situationen gegen das zu verstoßen, was wir eigentlich postulieren, und uns selber nicht daran halten. Und jeder bzw. jede Studierende wird das sehr, sehr genau registrieren, und wir verlieren an Glaubwürdigkeit. Deshalb würde ich Haltung nie prüfen. Ich verlange auch nicht, dass Student*innen meine Haltung übernehmen. Sondern ich versuche sie zu vermitteln, indem ich sie lebe. Und da gibt es natürlich auch ein paar Sachen, gerade in der Rhetorik, oder auch in der Methodik und Didaktik, die ich eben einfach empfehle, auch als Techniken, oder als Formulierungshilfen, oder sonst irgendetwas.

Susanne: Man kann Haltung im Laufe des Lebens also noch erlernen.

Dirk: Natürlich kann man das lernen, selbstverständlich. Man kann es nicht lehren oder nur sehr begrenzt lehren, aber man kann es sehr wohl lernen.

Steffi: Menschen verändern sich. Und unsere Arbeit fördert Entwicklung. Einem 18-Jährigen zu sagen: „passt nicht, weil du in einem schwierigen Umfeld aufgewachsen bist" fände ich wirklich nicht förderlich.

Dirk: Mir fällt in diesem Zusammenhang das schöne Zitat von Gunther Schmidt ein, der mal sinngemäß gesagt hat: „Um einigermaßen man selber zu bleiben, muss man sich ständig verändern. "Ich glaube, das ist tatsächlich so. Und ich verweise mal ganz frech auf mein Lehrbuchkapitel zur Didaktik und Methodik in der Rhetorik (Meyer, 2016, 146ff.). Da ist der erste Punkt ein humanistisches Menschenbild, und was ich unter diesem humanistischen Menschenbild subsummiere, hab ich da auch aufgeführt. Und das ist etwas, was ich unseren Student*innen in der Didaktik und Methodik immer gleich am Anfang vermittle. Für mich ist es die Grundlage. 
Susanne: Können wir nochmal zu den Techniken zurückkommen? Was sind weitere Verfahren für eine sprechwissenschaftliche Beratung?

Stephanie: Mir fällt jetzt ganz spontan Feedback ein. Eigentlich gebe ich gar kein Feedback mehr, sondern stelle Fragen an die Person, die sich ein Feedback wünscht. Die Person feedbackt sich quasi selbst. Ich begleite den Prozess nur noch. Mein Blick auf Feedback hat sich also verändert und das passiert mit allen Techniken, die wir im Studium gelernt haben: Wir machen Erfahrungen mit ihnen, neues Wissen kommt hinzu und dann verändern wir die Techniken.

Susanne: Wenn es um Feedback geht, habe ich sehr, sehr oft in verschiedenen Kontexten erlebt, dass die Leute sagen: „Ja, Sie brauchen mich danach jetzt nicht zu fragen, das kann ich Ihnen nicht sagen. Weil, ich beherrsche nicht die Technik mich selbst zu beobachten. Ich kann Ihnen nicht mehr sagen, ob ich die Leute angekuckt habe, denn ich war da so in dem Prozess drin. Ob ich da Pausen gemacht habe, keine Ahnung." Also ich glaube, eine wesentliche Technik ist die Eigenbeobachtung und zwar über verschiedene Sinne und Kanäle. Die Fähigkeit zur Selbstbeobachtung ist etwas, was wir in der Sprechwissenschaft in verschiedensten Stellen lehren.

Dirk: Absolut, also da stimme ich dir völlig zu. Und das ist auch für mich in den letzten Jahren ein regelrechter Schwerpunkt in der Rhetorik: die Konfrontation der Studierenden mit sich selbst. Und zwar in unterschiedlichen Kontexten. Dass sie also immer wieder sich selbst reflektieren, dass sie einander Feedback geben und sich Feedback holen. Das ist mir wirklich wichtig. Und wir haben ja in der Sprechwissenschaft den großen Vorteil, dass die Student*innen sich in so unterschiedlichen Bereichen selbst erfahren. Z. B. im Körper-Stimm-Training auch so etwas wie Körperhaltung reflektieren, zumindest sich damit auseinandersetzen. Und auch dann fällt mir wieder ein schöner Satz von Gunther Schmidt ein, der in einem Workshop gesagt hat: „Wie einer geht, so geht es ihm, und wie es einem geht, so geht er". In der Art und Weise, wie wir stehen, wie wir gehen, wie wir uns bewegen, drückt sich ja auch unser Befinden aus und das wirkt sich auf unsere Kommunikation aus. 
Susanne: Auch Stimmarbeit selbst bewirkt etwas an der Haltung. Anna Merkel hat im Rahmen ihrer Diplomarbeit in der Psychiatrie klassisches Stimmtraining, mit Patient*innen mit ängstlich-vermeidenden Persönlichkeitsstilen gemacht, also Übungen zur Stimme, Haltung, Lautstärke usw. Sie konnte dadurch psychische Ressourcen aktivieren.

Dirk: Klassisches Embodiment!

Steff: Ja, durch die Arbeit am Außen, an der Wirkung, am Ausdrucksvermögen passiert unglaublich viel innere Entwicklung. Zumindest wenn die Leute das zulassen und offen dafür sind. Das wirklich Spannende am Embodiment ist jedoch, dass es andersherum auch funktioniert. Embodiment heißt ja: Körper beeinflusst Psyche UND Psyche beeinflusst Körper. D.h. wenn beispielsweise die Arbeit an der äußeren Stimme stagniert, es sich lohnt, an der inneren Stimme zu arbeiten. Und dadurch tut sich meist auch etwas im Außen.

Susanne: Und das ist das Schöne an unserem Fach! Dass wir zu einem Gegenstand arbeiten, der eben beides verbindet, Körper und Seele, dass man Sprechen und Stimme von beiden Seiten betrachten und angehen kann. Es ist auch manchmal herausfordernd, diese Verantwortung zu spüren, von welcher Seite nähere ich mich jetzt dem Problem? Und dann immer wieder auch selbstkritisch zu fragen, ist das der richtige Weg? Das auch zu lernen. Das ist das Schöne an unserem Studium, an unserem Beruf. Einerseits ganz viel Wissen und andererseits auch ganz viel Erfahrung, ein Leben lang.

Dirk: Arbeit am Symptom oder an der Ursache. Auch das ist für mich eine Frage der Haltung, die ich meine und die ich anstrebe. Ich hab das vor vielen, vielen Jahren mal in einem Artikel verwendet, ein Zitat von Alexander Mitscherlich: Es wäre wichtig, dass „wir uns ... zu der Einsicht bequemen, wir könnten überall dort einem Wahn verfallen sein, wo wir meinen, etwas sei ,felsenfest' sicher." (Mitscherlich, A. 1968,157) Und ich glaube gerade in unserem Fach ist dieser Wahn, dass wir glauben irgendetwas ganz genau zu wissen, sehr gefährlich. Und wir sollten alle zu dieser „Demut“ kommen, zu sagen, ok, wir haben es mit einem sehr, sehr komplexen Gegenstand zu tun. Wir können in 
vielen Bereichen nicht felsenfest Sicherheit haben, zu wissen worum es geht oder was das Problem ist, oder wie man das Problem löst. Wir müssen da aus meiner Sicht wirklich demütig rangehen. Auch da wieder ein schönes Zitat aus dem Konstruktivismus, Heinz von Foerster hat mal gesagt: „Die ,hard sciences' sind erfolgreich, weil sie sich mit den, soft problems' beschäftigen; die ,soft sciences' haben zu kämpfen, denn sie haben es mit den , hard problems zu tun." (Foerster, H. v. 1993, 161). Und das ist, glaub ich, auch wirklich so.

Susanne: Gern würde ich noch folgende Frage an Stephanie richten. Wir haben ja seit dem 11.11.2019 die sprechwissenschaftliche Beratungsstelle. Jetzt gibt es schon eine ganze Menge Anfragen. Kannst du mal kurz erzählen, was das bisher für Anfragen sind?

Stephanie: Die Anfragen sind bunt und vielfältig. Und alles hat mit der Sprechwissenschaft zu tun. Das finde ich erst mal richtig eindrucksvoll. Dass bisher noch keine Frage da war, bei der ich dachte „Oh, das betrifft gar nicht unser Fachgebiet“. Das beantwortet vielleicht schon die letzte Frage, die du gestellt hast. Und was waren das für Anfragen? Anfragen zu phonetischen Themen, zum Beispiel von Nicht-Muttersprachler*innen, die ihre Aussprache verbessern wollen. Dann gab es Themen zur Stimme, „Meine Stimme ist zu leise, ich habe Beschwerden mit meiner Stimme, ich muss Vorträge halten" etc.. Es gab auch Fragen zu Vortragsthemen, wie „ich bin nervös oder ich habe Lampenfieber, wie kann ich das verändern, ich bin zu leise, man hört mich nicht“. Aktuell gab es auch das Thema: „ich werde nicht verstanden im Gespräch, ich glaube, ich spreche zu schnell“. Also das sind ganz unterschiedliche Themen, die wir aber alle gut bedienen können. Dann gab es zum Beispiel noch einen Kollegen aus der Uni, der hatte Fragen zur Sprachentwicklung gestellt, weil er gerade zwei kleine Kinder hat.

Dirk: Und das ist übrigens etwas, was ich unseren Student ${ }^{*}$ innen auch immer wieder sage, wenn ich, wie zum Beispiel jetzt gerade in der Wirtschaftsrhetorik im Master, mit ihnen zusammenarbeite und sie natürlich auch große Ängste haben: „Wenn ich dann später auf dem ,freien Markt` arbeiten will, mit wem habe ich denn da zu tun, wenn ich da in der Wirtschaft arbeite?“. Ich versuche 
ihnen immer wieder klar zu machen: „Natürlich ist der Markt unheimlich voll und da tummeln sich alle möglichen Leute. Aber der große Vorteil, den ihr habt als Sprechwissenschaftler*innen, dass ihr in einer solchen Breite über das Sprechen und die Stimme, über Aussprache und über Phonetik und über physiologische Grundlagen usw. Kenntnisse und Wissen habt, das viele Mitbewerber*innen in der Rhetorik auf dem Markt eben nicht vorweisen können. Das ist ein großer Vorteil, den ihr habt. Ihr habt eine fundierte Grundlage. Auf die könnt ihr möglicherweise nicht in gleichem Maße immer zurückgreifen, aber sie ist da. Und wenn ihr euren Fokus darauf setzt, dann werdet ihr da auch schnell wieder fündig werden und deswegen auch auf diesen Gebieten aussagekräftig sein." Das finde ich großartig und das sollten wir unseren Studierenden immer wieder klar machen.

Steffi: Ja, ich kann das bestätigen, was Dirk sagt. Unser Hauptmerkmal ist dieses interdisziplinäre, vernetzte Denken von allen möglichen Bereichen des Sprechens. Genau das, was es so schwer macht, die Ausbildung auszubalancieren, ist später letztlich unser Wettbewerbsvorteil. Ich denke, unser Manko ist, dass wir lernen müssen, dieses Selbstverständnis nach außen hin deutlicher zu positionieren. Dass es da einen beratenden Berufsstand gibt, der gegenüber den selbsternannten Rhetorik-Gurus seine Anerkennung verdient hat. Wenn ich mich als Sprechwissenschaftlerin fernab des universitären Kontextes vorstelle, weiß keiner, was ich tue. Als Auftritts-Coach schon. Das ist aber kein geschützter Begriff. So kann sich Jeder nennen. Es herrscht also noch Aufklärungsbedarf.

Susanne: Und darum waren die Kleine Fächer-Wochen Sprechwissenschaft ja auch so wichtig. Wir haben uns dabei, glaub ich, auch nochmal wieder selbst vergewissert, wir haben uns aber selber auch neu kennengelernt und wir haben viele aktuelle Themen mutig angefasst. Und wir haben da auch viel Energie mitgenommen, um deutlicher zu sagen, wer wir eigentlich sind.

Folgende Frage möchte ich Euch stellen, weil deren Beantwortung jetzt nach diesem Onlinesemester infolge der Covid-19-Pandemie drängt. Kann man sprechwissenschaftlich beraten in einer Zeit, wo eigentlich Präsenz nicht möglich ist? Ist es möglich über Konferenz-Tools, Telefon und sonstige Wege 
eine Beratungssituation herzustellen, die Vertrauen schafft, die alles aufnimmt, was wichtig wäre, um jemanden angemessen zu beraten?

Dirk: Ich glaube nicht, dass man das kann. Da komme ich zu dem Punkt der digitalen Lehre. Da kommt ja immer mal wieder so ein Gedanke, naja, es ist ja besser als nichts. Und die Studierenden lernen ja auch da etwas. Ja ok, dass meine Student*innen etwas lernen, der Anspruch, den ich an meinen Beruf habe, und das genügt mir nicht, wenn sie „irgendwas“ lernen. Man kann Beratung natürlich auch online machen, auch in unserem Bereich, und das ist besser als nichts. Aber dass das ein gleichwertiger Ersatz zur face-to-face-Situation ist oder werden kann, irgendwann mal, daran glaube ich nicht.

Steff: Du hast vorhin ja zwei Fragen gestellt. Einmal, kann diese Online-Beratung Vertrauen aufbauen? Ich denke, das kann sie. Meine Erfahrung hat mir gezeigt, dass auch online eine Vertrauensbasis entstehen kann. Und das zweite ist, kann ich alles erfassen? Das kann ich nicht. Definitiv nicht. Das muss uns allen bewusst sein. Ganz viele Informationen, z. B. über Körpersprache, bekomme ich nicht mit. Das auch zum Gegenstand zu machen, macht auch gute Beratung aus. Im Sinne von Transparenz. Live-Situationen sind ein anderer Kontext und einfach nicht vergleichbar.

Susanne: Dirk, noch eine Frage. Du hast jetzt Gunther Schmidt erwähnt ...

Dirk: Ja, Gunther Schmidt leitet ja die sysTelios Klinik in Heidelberg für psychosomatische Erkrankungen usw. Und da erzählte er in einem Workshop, dass er immer die neu in eine Gruppe ankommenden Patient ${ }^{*}$ innen begrüßt. Und zwar heißt er sie herzlich willkommen sagt ihnen, dass sie natürlich alle nicht wegen ihrer Probleme da sind. Es ist ja niemand da, weil er Angst hat, oder so, sondern weil er eben keine Angst mehr haben will. Weil er einen $\mathrm{Zu}-$ stand will, der anders ist als der, den er jetzt hat. Deswegen ist niemand wegen seines Problems hier, sondern weil er die Lösung für sein Problem sucht und erhofft. Und ich glaube, dass das vielleicht auch so eine Grundhaltung ist, die man für so eine Beratungsstelle ganz gut gebrauchen kann. Die Leute kommen ja nicht hierher, weil sie ein Problem haben, sondern sie kommen hier her, weil 
sie hoffen, dass ihnen hier in irgendeiner Art und Weise dabei geholfen wird, ihr Problem zu lösen.

Susanne: Habt vielen Dank für das interessante Gespräch. Ich fasse mal zusammen, dass jemanden gut zu beraten letztendlich heißt, ihn oder sie darin zu unterstützen die eigenen Potentiale aufzudecken. Und dazu bedarf es der Haltung, weniger Ratschläge zu erteilen als vielmehr explorierende Fragen zu stellen.

Wir danken Stefanie Prochazka für die Unterstützung bei der Verschriftlichung des Interviews.

\section{Literatur}

De Shazer, Steve (2019): Der Dreh. Überraschende Wendungen und Lösungen in der Kurzzeittherapie. Heidelberg: Carl-Auer.

Foerster, Heinz von (1993): KybernEthik. Berlin: Merve.

Foerster, Heinz von (2009): Entdecken oder Erfinden. Wie lässt sich Verstehen verstehen? In: Gumin, Heinz/Meier, Heinrich (Hg.): Einführung in den Konstruktivismus Bd. 5. München: Piper, S. 41-88.

Merkel, Anna/Voigt-Zimmermann, Susanne (2011): Potential von Stimmübungen zur Aktivierung psychischer Ressourcen - eine qualitativ-empirische Studie. In: Bose, Ines/Neuber, Baldur (Hg.): Interpersonelle Kommunikation: Analyse und Optimierung. Hallesche Schriften zur Sprechwissenschaft und Phonetik Bd. 39. Frankfurt am Main: Peter Lang, S. 261-268.

Meyer, Dirk (2016): Didaktik und Methodik in der Rhetorik. In: Bose, Ines/Hirschfeld, Ursula/Neuber, Baldur/Stock, Eberhard (2016): Einführung in die Sprechwissenschaft. Phonetik, Rhetorik, Sprechkunst. Tübingen: Narr, S. 146-153.

Mitscherlich, Alexander und Margarete (1968): Die Unfähigkeit zu trauern. Grundlagen kollektiven Verhaltens. München: Piper.

Schmidt, Gunther (2014): Einführung in die hypnosystemische Therapie und Beratung. Heidelberg: Carl-Auer. 


\section{Internetquellen}

Internetquelle 1: https://kfw.sprechwiss.uni-halle.de/eroeffnung-dersprechwissenschaftlichen-beratungsstelle/ (Letzter Zugriff 17.02.2021) 



\section{"Comisch“ - Coaching mit Schauspielern als konsequent klientenzentrierte und lösungsfokussierte Methode zur partnerorientierten Gesprächsrhetorik ${ }^{1}$}

Zusammenfassung: Der Name des Coachingtools "Comisch" ist Abkürzung und Wortspiel zugleich. Einerseits steht es für Coaching mit Schauspielern, andererseits können die Ergebnisse der Arbeit durchaus als komisch im Sinne von lustig, seltsam oder eigenartig verstanden werden. Coach und Schauspieler bilden ein Team, das sich lösungsorientiert und klientenzentriert mit dem Coachee auf die Suche nach seiner Lösung einer schwierigen Situation macht.

Abstract: The name of our coaching tool "comisch" is an abbreviation and a play of words at the same time. On the one hand, it incorporates the German acronym Coaching mit Schauspielern. On the other hand, it stands for komisch which means funny, weird, or odd. Together with their coachee, coach and actor build a solution focused and client-centered team on a quest for the client's best solution of a difficult situation.

„Comisch ... nein, nicht mit „ $k$ “, sondern mit „ „c“... comisch“. „Ja, lustig kann es auch werden, meistens ist es aber eher ernst“. „Nein, es ist keine Methode zum Coaching von Schauspielern“!

So lauten mit Augenzwinkern die Standardantworten auf Reaktionen von Menschen, die ein erstes Mal von Comisch hörten. Mit diesem Artikel möchte ich gerne erklären, wie diese Methode entstand, wie sie funktioniert und in welchen Anwendungsgebieten sie hilfreich sein könnte.

1 Aufgrund der besseren Lesbarkeit wird im Text das generische Maskulinum verwendet. Gemeint sind jedoch immer alle Geschlechter. 


\section{$1 \quad$ Die Entstehung}

\section{Inspiration}

Im Rahmen einer Weiterbildung zum Systemsichen Coach an der CoachingAkademie Köln begegnete ich erstmals der Methode des leeren Stuhls. Sie kommt dann zum Einsatz, wenn sich im individuellen Coachingprozess herausstellt, dass ein Klient einen ungelösten Konflikt mit einer Person im näheren Umfeld hat und ein klärendes Gespräch hilfreich sein könnte. Wenn der Coachee eine grobe Einschätzung über das Verhalten der betreffenden Person abgeben kann, dann kann eine simulierte Aussprache einen Lösungsprozess in Gang bringen.

Seinen Ursprung hat der ,leere Stuhl' in der Gestalttherapie, wo die Methode auch als Zwei-Stuhl-Technik bezeichnet wird (vgl. dazu Hartmann-Kottek 2004, 220ff.). Hierzu stellt der Klient zwei Stühle gegenüber auf und wird vom Coach durch einen vierstufigen Prozess geführt.

\section{- Präparation und Einfühlung}

In der Präparationsphase stellt der Klient die beiden Stühle einander gegenüber, um eine konfrontative Gesprächssituation zu simulieren. Danach bittet der Coach den Klienten, sich eine Situation zu vergegenwärtigen, in der das nachgestellte Gespräch stattfinden könnte. Je genauer die Definition von Ort, Zeit, Konfliktpartner und Raum ist, desto leichter wird sich der Coachee in der anschließenden Projektionsphase tun. Wichtig ist die mentale Vorbereitung des Klienten auf die Imagination des Gegenübers auf dem gegenüberliegenden Stuhl. Nach erfolgter Situierung fühlt sich der Klient ein und nimmt auf ,seinem' Stuhl Platz, sobald er sich dazu bereit fühlt.

\section{- Projektion und Selbstexploration}

In der zweiten Phase des Prozesses lädt der Coach den Klienten ein, seinem Gegenüber ungefiltert alles zu sagen, was ihm in den Sinn kommt. Auf Gepflogenheiten des guten Umgangs kann gezielt verzichtet werden, um eine Exploration der Gefühle zu ermöglichen. 
Dennoch ist auf die Vermeidung einer emotionalen Durchflutung zu achten (vgl. ebd., 215). Ist die erste Welle der Äußerung beendet, bittet der Coach den Klienten, sich kurz hinter seinen Stuhl zu begeben und aus dissoziierter Sicht die Situation zu betrachten. Falls dem Klienten noch etwas wichtig ist, kann er sich wieder setzen und seinem Gegenüber nun weitere Wahrnehmungen und Eindrücke mitteilen. Der Vorgang der Dissoziation und Re-Assoziation wird so oft wiederholt, bis der Klient wirklich alles sagen konnte. In dieser Phase ist zu beobachten, dass mit jeder Dissoziation die Wahrnehmung des Konfliktpartners und der Situation differenzierter wird. Demzufolge werden auch die Äußerungen meist emotional weniger konfliktbehaftet und wertschätzender.

\section{- Rollentausch}

Nach vollzogener Projektion und Selbstexploration wird der Klient eingeladen, sich auf dem Weg zum gegenüberliegenden Stuhl in sein Gegenüber zu verwandeln. Sobald sich der Klient in sein Gegenüber eingefühlt hat, spricht der Coach den Klienten mit dem Namen des Konfliktpartners an und bittet ihn, auf dem bislang leeren Stuhl Platz zu nehmen. Schon an dieser Stelle zeigt sich an den Pentimenti des Klienten, ob Einfühlung und Verwandlung stattgefunden haben. So kommen bei gelungener Metamorphose beispielsweise Gesichtszüge, Laute oder auch typische Gesten des Gesprächspartners zum Vorschein, ohne dass das vom Klienten beabsichtigt wird. Der Coach moderiert die Situation und lädt den Klienten, nun in der Position des Gegenübers ein, auf die Äußerungen zu antworten, die er vorher als er selbst getätigt hatte. In einer Rückprojektion auf sich selbst kann sich der Klient nun aus der anderen Perspektive äußern. Nach erfolgter erster Welle wird auch hier die Methode der Dissoziation und der Re-Assoziation verwendet, wobei der Coach den Klienten konsequent mit dem Namen des Gesprächspartners anspricht. Dieser Vorgang wiederholt sich so lange, bis der Klient alles anbringen kann, was ihm in dieser Situation wichtig ist. 


\section{- Rücktausch und Versöhnung}

Nach Beendigung der dritten Phase verwandelt sich der Klient wieder zurück in sich selbst, ohne nochmals auf dem ersten Stuhl Platz zu nehmen. Er bleibt also im dissoziierten Zustand. Nun kann mit dem Klienten besprochen werden, ob sich durch die Verwandlung in der Wahrnehmung des Konfliktes etwas verändert hat. Meist ist das der Fall, sodass sich mit dem Perspektivwechsel ein differenzierteres, oft auch versöhnlicheres Empfinden der Beziehung eingestellt hat. Im Anschluss an den leeren Stuhl kann mit Klienten ein für ihn geeigneter Umgang mit der Situation besprochen werden.

\section{Was wäre, wenn ...?}

Nach Beendigung der Weiterbildung hatte ich die Gelegenheit, in vielen Einzelsitzungen mit Klienten die Methode auszuprobieren. Da sich die Anliegen der Coachees meist auf konkrete, in naher Zukunft stattfindende Gesprächssituationen bezogen, konnte Erfolg oder Misserfolg der erarbeiteten Strategien in der Coachingsituation selbst nicht direkt erprobt werden. Basierend auf meiner langjährigen Erfahrung als Ausbilder an Schauspielschulen und als Theaterregisseur kam mir der Gedanke: Was wäre, wenn sich auf dem leeren Stuhl gegenüber tatsächlich jemand befände? Schauspielerinnen und Schauspieler sind in besonderem Maße befähigt, mit ihrem Können die Methode des leeren Stuhls sprichwörtlich mit Leben zu erfüllen. Einerseits verfügen sie durch das intensive Beobachtungstraining über die notwendigen rezeptiven Fähigkeiten, andererseits sind sie durch ihre Improvisationsstudien spontan im Stande, wahrgenommene Verhaltensweisen recht detailgetreu zu wiederholen. Gleichzeitig sind sie wie keine andere Berufsgruppe in der Lage, schnell in eine Figur zu schlüpfen und diese konsistent in einer unbekannten Situation zu spielen.

Nach der Lektüre hypnosystemischer Arbeiten von Milton Erickson und eigenen phänomenologischen Erfahrungen mit systemischer Aufstellungsarbeit entwickelte ich zusammen mit meiner Partnerin Christin Alexandrow, selbst Schauspielerin und diplomierte Mentalcoach, ein Ausbildungscurriculum. Mit fünf Schauspielerinnen und Schauspielern aus Berlin und Bayern fand 
in Regensburg und Leuchtenberg in der Oberpfalz ein insgesamt sechs Tage dauernder Entwicklungsworkshop statt. Das Ergebnis dieses Ausprobierens stellt den jetzigen Stand der Methode Comisch dar.

\section{Die Methode Comisch im Überblick}

Bei Comisch handelt es sich um einen zweifach erweiterten Prozess des, leeren Stuhls'. Erstens ist neben Coach und Klient noch ein Schauspieler involviert, der jeweils auf dem Stuhl gegenüber Platz nimmt. Zweitens erhält der Klient durch die Anwesenheit eines sogenannten Comisch-Actors die Gelegenheit, den Prozess mehrfach zu durchlaufen. Dadurch besteht die Möglichkeit, Erfolg oder Misserfolg der gewählten Strategie durch eine Veränderung von Situation oder gezeigtem Verhalten in einem weiteren Durchgang sofort auszuprobieren und zu erleben.

Grundsätzlich ist Comisch in einen konsequent klientenzentrierten und lösungsfokussierten Coachingprozess eingebettet. Vor der Anwendung der Methode findet durch den Coach eine Klärung des Anliegens und der Zielsetzung statt. Zugleich führt der Coach den Coachee in einen ressourcenvollen Zustand, der ihm die Suche nach Lösungen erleichtert. Die Aufgabe des Coaches besteht darin, den Klienten durch systemische Fragen zu eigenen Lösungen zu führen, die außerhalb seines Problemerlebens liegen. Tipps oder Ratschläge durch den Coach werden hier nicht gegeben (vgl. dazu Sparrer 2017, de Shazer 2012, de Shazer/Dolan 2015).

\section{Situierung}

Bis zum eigentlichen Comisch-Prozess ist der Comisch-Actor nicht im Raum, um unvoreingenommen seine Arbeit verrichten zu können.

Die erste Stufe des Comisch-Prozesses selbst besteht aus einem Briefing, das der Klient dem Comisch-Actor gibt. Inhalt dieser kurzen Vorbesprechung sind Fragen über Alter, Familienstand, Geschlecht, Hobbies oder berufliche und gesellschaftliche Stellung des darzustellenden Gegenübers. Die Steuerung des Briefings liegt beim Comisch-Actor. In dieser für ihn sehr wichtigen Phase 
sammelt er alle für die Darstellung relevanten inhaltlichen Daten. Fragen, die eine subjektive Bewertung des Gegenübers durch den Klienten triggern könnten, werden bewusst vermieden. Die Vorab-Befragung endet mit der Klärung der Situation, in der das nachfolgend simulierte Gespräch stattfinden soll.

\section{Projektionsphase}

In der Projektionsphase führen Klient und Schauspieler das Gespräch so, wie es der Coachee für richtig und angemessen hält. Der Comisch-Actor vollbringt in dieser Phase Schwerstarbeit, da es sich um eine hochkomplexe Aufgabe handelt:

- er fühlt sich in die Situation ein, die ihm zu diesem Zeitpunkt größtenteils unbekannt ist,

- er bahnt sich darstellerisch einen Weg zum Gegenüber des Klienten, das er sich aus den wertungsfreien Briefingfragen und improvisatorisch aus dem Verhalten des Klienten erschließen muss,

- er beobachtet das Verhalten des Klienten in der Situation und speichert verbale, paraverbale und nonverbale Eindrücke. Am wichtigsten ist für die Beobachtung des Klienten aber der emotionale Eindruck, den der Comisch-Actor vom Klienten gewinnt.

Durch diese Komplexität kommen für diese Methode nur Schauspieler infrage, die sich voll in den Dienst des Coachee stellen können und nicht die Steuerung der Szenerie übernehmen. Darsteller mit übersteigertem Sendungsbewusstsein sind für Comisch nicht geeignet. Die Hauptaufgabe für den Comisch-Actor besteht während der Projektionsphase darin, dem Klienten im übertragenen Sinn als „Leinwand“ für seine Lesart des Gegenübers zu dienen. Der Schauspieler gleitet improvisierend in die Situation, nimmt die diskursiven Impulse des Klienten auf und verarbeitet sie im Rahmen des vereinbarten Settings als Gesprächspartner des Coachee.

Anders als in der Methode des, leeren Stuhls' hat der Klient nun eine reale Person vor sich, die auch antwortet. Mit jedem Moment wird die Situation detailgetreuer, da der Comisch-Actor immer mehr Eindrücke aus dem Verhalten des Klienten sammelt, die er für seine Darstellung verwenden kann. Das wie- 
derum sorgt dafür, dass sich der Klient nach und nach in einen tranceartigen Zustand versetzt, sodass die Situation aus seinen Augen real erscheint. Dabei ist es nicht relevant, ob der Schauspieler tatsächlich dem Geschlecht oder dem Alter des Gegenübers entspricht. In einem Comisch-Prozess sprach eine Klientin ihr in der Ursprungssituation männliches und von Christin Alexandrow dargestelltes Gegenüber mehrfach mit Namen an, obwohl sie den eigentlich nicht nennen wollte.

Aufgabe des Coaches ist es indes, das Gespräch aufmerksam zu beobachten und den richtigen Zeitpunkt zu finden, um die Sequenz zu beenden. Oberste Prämisse ist es, dass der Comisch-Actor genug ,Material' für die nachfolgende Spiegelung sammeln konnte.

\section{Spiegelung}

Für die Spiegelung tauschen die beiden Gesprächspartner die Positionen und wiederholen den eben geführten Dialog mit vertauschten Rollen. Der Comisch-Actor zeigt dem Klienten, was er emotional und auch äußerlich wahrgenommen hat. In der Darstellung legt er den Fokus auf Trigger eigener emotionaler Reaktionen, die Handlungen oder Äußerungen des Klienten während der Projektionsphase bei ihm ausgelöst haben. Wenn er zum Beispiel passive Gewalt anhand einer deutlichen körperlichen Abwendung und einer entsprechenden Äußerung wahrgenommen hat, wird er das dem Klienten während der Spiegelung zeigen.

Der Coach sorgt durch eine einfühlsame Moderation während des Übergangs von der Projektion zu Spiegelung dafür, dass sowohl der Klient als auch der Comisch-Actor den Prozess fokussiert durchleben können. Hierzu bittet der Coach, die bisherige Position zu verlassen, kurz durchzuatmen und dann den Weg zur Stelle des Gegenübers anzutreten. Dabei leitet der Coach die beiden an, sich in die andere Person zu verwandeln und nach vollzogenem Einfühlen das Gespräch aus der Warte des Gegenübers erneut zu führen. Während der Spiegelung selbst hält sich der Coach wieder zurück und findet den rechten Moment, den Diskurs zu unterbrechen. Mit der Unterbrechung verlässt der Schauspieler wieder den Raum. 
Für den Coachee ist die Spiegelung die eindrücklichste Phase der Methode, da er sein eigenes Verhalten nicht nur beobachtet, sondern am eigenen Leib erlebt. Wie schon in der Projektion taucht er in einen Trancezustand, der ihn sich selbst aus der Perspektive seines Gegenübers erleben lässt.

\section{Eigenfeedback}

Der Klient bleibt im Gestus seines Gegenübers und gibt anschließend „sich selbst“ Feedback. Um das Erlebnis für den Coachee so eindrücklich wie möglich zu gestalten, benutzt der Coach die Technik der kataleptischen Hand. Hierbei handelt es sich um ein Verfahren aus der systemischen Aufstellungsarbeit, um eine körperlich nicht anwesende Person für einen Klienten zu vergegenwärtigen (vgl. dazu Internetquelle 1). Der Coach wird darauf achten, dass der Klient in der Rolle seines Gegenübers verharrt und die Selbstexploration aus dessen Position heraus formuliert. Durch seine Moderation leitet der Coach den Klienten zu einem Feedback nach der allgemein anerkannten WWW Formel für Wahrnehmung, Wirkung und Wunsch. Für den Coachingprozess ist der Moment des Eigenfeedbacks entscheidend. Daraus entwickelt der Klient, noch immer in der Position des Gegenübers, Maßnahmen, die für eine zweite Spiegelung und auch ein reales Gespräch hilfreich sein könnten. Folgende Fragen haben sich in diesem Stadium des Comisch-Coachings als günstig erwiesen:

- (Name des Gegenübers) - mal angenommen, es gäbe die Möglichkeit, das Gespräch nochmals durchzuführen - was sollte (Name des Klienten) aus Ihrer Sicht anders machen?

- Worauf sollte (Name des Klienten) unbedingt achten, um Sie (ggf. Name des Gegenübers) zu überzeugen/mitzunehmen/achten/etc.

- (Name des Gegenübers), was sollte (Name des Klienten) besser unterlassen?

Der Coach beendet das Feedback mit folgender Abschlussfrage: „(Name des Gegenübers), war das alles, was Sie (Name des Klienten) als Feedback zu dem Gespräch geben wollten? Gibt es noch etwas?“. Falls dem so sein sollte, wird 
das dem Klienten ermöglicht. Jede Äußerung und jede Erkenntnis hat das Potenzial, einen wertvollen Handlungsimpuls zu liefern. Gerade hier zahlt sich eine Hartnäckigkeit des Coaches im Prozess aus.

\section{Rücktausch, zweiter Durchgang oder Beendigung des Prozesses}

Der Coachee verwandelt sich nun wieder zurück in sich selbst und begibt sich auf seine ursprüngliche Position. Der Coach fragt nun: „(Name des Klienten), Sie haben gehört, was (Name des Gegenübers) gesagt hat. Wie geht es Ihnen damit?" Der Klient äußert sich zu seinen Wahrnehmungen und Eindrücken und bespricht mit dem Coach, ob eine Wiederholung des Gespräches in Form einer zweiten Spiegelung stattfinden soll oder der Prozess an dieser Stelle beendet ist. Sollte es zu einer zweiten Spiegelung kommen, klären Coach und Klient die Änderungen in Setting und Verhalten und rufen den Comisch-Actor zu einem zweiten Durchgang. Erfahrungsgemäß ändern sich die Umgebung des Gespräches und auch die innere Haltung zum Gesprächspartner. So können beispielsweise konfliktäre Gespräche aus einem nüchternen Meetingraum in einen Spaziergang an der frischen Luft verändert werden und der Klient benennt erst wertschätzende Aspekte für die Beziehung mit dem Gegenüber, um erst dann auf kritische Punkte zu kommen. Der Prozess beginnt unter Beachtung der Veränderungen von neuem und der Schauspieler wird wieder hereingerufen. Das Briefing wird auf ein Mindestmaß an Hinweisen zu geänderten äußeren Rahmenbedingungen gekürzt und der zweite Durchgang beginnt. Durch die veränderte Projektion und die darauffolgende Spiegelung bekommt der Klient einen lebendigen Eindruck, inwieweit die vorgenommenen Abwandlungen Einfluss auf Verlauf und Ergebnis des Gespräches haben können. Theoretisch kann der Vorgang so oft wie nötig wiederholt werden, allerdings hat noch kein Comisch Prozess mehr als drei Projektionen, Spiegelungen und Eigenfeedbacks erlebt. 


\section{Compliments}

Am Ende der Sitzung geben Coach und Schauspieler dem Klienten wertschätzende Wahrnehmungen mit auf den Weg, sogenannte Compliments. Ihr Zweck ist es, den Coachee in einem ressourcevollen Zustand ins Außen zu verabschieden. Darin werden explizit nicht die Mitarbeit des Klienten in der Sitzung und die Kreativität im Prozess selbst hervorgehoben, sondern die Ressourcen, die ihm beim Lösen des Konfliktes helfen können. Der Coach wird sich auf Äußerungen des Klienten aus der lösungsorientierten Situierung und aus der Phase des Rücktausches stützen, der Schauspieler auf Wahrnehmungen, die die Empathiefähigkeit des Coachees während der Projektion und in der Spiegelung in den Fokus nehmen. Hier einige Beispiele:

- „Mir ist aufgefallen, dass Ihnen die Beziehung zu (Name des Gegenübers nennen) trotz der schwierigen Situation wichtig ist, da Sie (Beispiel nennen).

- Ich finde es sehr beachtlich, dass Ihnen Sie für eine Lösung des Konfliktes bereit sind, sich auf unbekanntes Terrain zu begeben, da Sie (Beispiel nennen)

- Ich bin beeindruckt, wie sehr Sie das Große Ganze im Auge haben, da Sie ja (Beispiel nennen):

- Etc.

Die Compliments sind der einzige Moment im gesamten Coachingprozess, in dem das Comisch-Team eine eigene Wertung vornimmt und äußert. Sie dient einzig dazu, im Klienten das Gefühl zu verstärken, eine eigene Lösung $\mathrm{zu}$ finden und die als schwierig wahrgenommene Situation zu meistern. Mit diesen Eindrücken wird der Klient verabschiedet.

\section{Anwendungsgebiete für Comisch}

Es zeigte sich, dass insbesondere im Bereich der Wirtschaftsrhetorik ein Interesse an dieser Methode besteht und typische berufliche Kommunikations- 
situationen wie Verhandlungen, Konfliktgespräche, Jobinterviews, Servicequalifizierungen, Umgang mit kritischen Kunden etc. individuell bearbeitet werden können. Besonders viel Nutzen entfaltet Comisch, wenn es in einen Workshop eingebunden wird, um Inhalte daraus sofort auf ihre Anwendbarkeit hin zu verproben. Auch für persönliche Anliegen im therapienahen Umfeld kann die Methode hilfreich sein, allerdings bedarf es einer umfänglichen organisatorischen Vorbereitung, was für Therapeuten einen häufig zu großen Aufwand bedeutet.

Grundsätzlich stellt Comisch einen Versuch dar, in Klienten induktive Lernprozesse aus dem eigenen Verhalten heraus zu triggern. Die konsequente Klientenzentrierung dient hierbei als oberste Maßgabe. Weder Coach noch Actor geben mit Ausnahme der Compliments eine Wertung ab. Beide stellen sich in den Dienst des Klienten im Vertrauen darauf, dass er über alle Ressourcen verfügt, die Herausforderung zu meistern.

Und bevor ich es vergesse: manchmal kann es dann doch komisch im Sinne von lustig zugehen, beispielsweise dann, wenn eine Klienten nach wenigen Sekunden in der ersten Spiegelung in schallendes Gelächter ausbricht und mit Tränen in den Augen ausruft: „Wie kann ich nur so blöd sein und dieses Gespräch überhaupt in Erwägung ziehen? Danke, Sie haben mir sehr geholfen!"

\section{Literatur}

Hartmann-Kottek, Lotte (2004): Gestalttherapie. Berlin: Springer.

Sparrer, Insa (2017): Einführung in Lösungsfokussierung und systemische Strukturaufstellung. 4. Auflage, Heidelberg: Carl-Auer-Systeme Verlag.

de Shazer, Steve (2012): Wege der erfolgreichen Kurztherapie. 11. Auflage, Stuttgart: Klett-Cotta.

de Shazer, Steve/Dolan, Yvonne (2015): Mehr als ein Wunder. Lösungsfokussierte Kurztherapie heute, 4. Auflage, Heidelberg: Carl-Auer.

Winter, Matthias (2017): Suche nicht nach Fehlern, erfinde Lösungen. In: Wagner, Roland (Hrsg.): Sprechen, Zeitschrift für Sprechwissenschaft, Sprechpädagogik, Sprechtherapie, Sprechkunst. Heft 63, S. 41-58.

Internetquelle 1: https://www.lexikon-aufstellungen.de/post/kataleptische-hand (Letzter Zugriff: 24.02.2021) 

Friderike Lange, Halle (SaAle)

\title{
Feedback als Haltung in der Lehrerinnenbildung - im Spannungsfeld zwischen Beraten und Bewerten
}

Zusammenfassung: In der Lehrer ${ }^{*}$ innenbildung ist das Feedback eine wesentliche und unverzichtbare Methode. Ein mit einem positiven Lerneffekt verbundener Feedbackprozess setzt eine achtsam und respektvoll gestaltete Beziehung sowie den reflektierten Einsatz des „Feedback als Haltung" voraus, um das Spannungsfeld zwischen Beraten und Bewerten in diesem Prozess auflösen zu können.

Stichworte: Feedback, Beraten, Bewerten, ko-konstruktiv, Reflexion

\begin{abstract}
Feedback is a central and indispensable method in teacher training. A feedback process associated with a positive learning effect presupposes a mindful and respectful relationship and the reflected use of feedback as an attitude to dissolve the tension between counseling and evaluating in this process.
\end{abstract}

Keywords: feedback, counseling, evaluating, co-constructive, reflection

$\mathrm{Zu}$ den vielen erfolgreichen Veranstaltungen der „Kleine Fächer-Wochen Sprechwissenschaft" 2019/20 an der Martin-Luther-Universität Halle-Wittenberg gehörte ein praxisorientierter Workshoptag mit insgesamt ca. 80 Teilnehmenden zum Thema „Feedbackkultur in der Lehrer*innenbildung“, der am 11. Januar 2020 in Halle stattfand. In diesem Rahmen wurde unter anderem ein Workshop zum Thema „Feedback geben - im Spannungsfeld zwischen Beraten und Bewerten" angeboten. Der vorliegende Beitrag erläutert das Konzept und die Ergebnisse dieses Workshops und schlägt damit einen Ansatz zur Auflösung des Spannungsfelds vor. 


\section{$1 \quad$ Einleitung}

Lehramtsstudierende und Lehrer*innen im Vorbereitungsdienst wünschen sich, Fehler machen zu dürfen. Sie wünschen sich, offen über ihre Unterrichtserlebnisse reflektieren zu können, ohne negative Folgen befürchten zu müssen. In der Lehrer*innenbildung tätige Personen wünschen sich ein vertrauensvolles Miteinander, in dem auch kritische Rückmeldungen konstruktiv aufgenommen werden. Wie kann eine solche Feedback-Kultur gestaltet werden, auf deren Basis Scheitern erlaubt, ja vielleicht sogar erwünscht ist, und ein achtsamer und (ko-)konstruktiver Lernprozess im Gespräch stattfinden kann?

Um Antworten auf diese Frage zu geben, wurde im Rahmen der „Kleine Fächer-Wochen Sprechwissenschaft" ein ganzer Tag mit Workshop-Angeboten $\mathrm{zu}$ verschiedenen Facetten von Feedback angeboten. Eingeladen waren Studierende, Lehrer*innen im Vorbereitungsdienst, Lehrkräfte im Schuldienst, Mentor*innen, Fachdidaktiker*innen, Haupt-und Fachseminarleiter*innen und alle, die sich für das Thema Feedbackkultur in der Lehrer*innenbildung interessierten. Das gesamte Spektrum der angebotenen Workshops kann auf der Internetpräsenz der Qualitätsoffensive Lehrerbildung (vgl. Internetquelle 1) nachgelesen werden.

\section{Warum spielt Feedback in der Lehrer*innenbildung eine zentrale Rolle?}

Sowohl im Rahmen der Praxisphasen innerhalb des Studiums (vgl. u. a. Pfeiffer 2019) als auch in der zweiten Phase der Lehrer*innenbildung, dem Vorbereitungsdienst bzw. Referendariat (vgl. u.a. Behnke 2016), reflektieren angehende Lehrer*innen im Rahmen von Unterrichtsnachbesprechungen ihre eigene pädagogische Praxis. Oftmals werden sie dabei von einem Gegenüber (Dozierenden, Seminarleiter ${ }^{\star}$ innen, Mentor ${ }^{\star}$ innen, Seminargruppe etc.) begleitet und erhalten in diesem Rahmen Feedbacks zu ihrem Auftreten, ihrer Wirkung, ihren Interventionen und Vorgehensweisen. Dabei wird immer wieder die Unsicherheit und Sorge geäußert, dass fachliche Schwächen oder Schwierigkeiten mit Schüler*innen kritische Anmerkungen der Fachleitungen evozieren und damit grundsätzlich negative Folgen für die angehenden Lehrer*innen hätten (vgl. Schledde 2007, 13). Dies geht mit dem Dilemma der Doppelrolle 
einher, in der sich begleitende Personen wie Fachleitungen und Mentor*innen befinden: Je nach Situation haben sie in den Unterrichtsnachbesprechungen sowohl eine beratende, aber oftmals auch gleichzeitig eine bewertende Funktion. Forschungsarbeiten wie die von Behnke (2016) zum Thema Feedback im Kontext Schule bestätigen diese Unsicherheiten: In ihrer Studie kommt Behnke zu dem Schluss, dass die aktuelle Feedbackpraxis im schulischen Bildungsbereich nicht annähernd ausreichend ausgeprägt ist und hier ein starker Fort- und Weiterbildungsbedarf besteht (vgl. ebd., 257).

Ausgehend von diesem Desiderat zur Feedbackkultur in der Lehrer*innenbildung wurde der Workshoptag im Rahmen der „Kleine Fächer-Wochen Sprechwissenschaft" ausgerichtet und mit großem Zuspruch angenommen.

Im Folgenden soll einer der in diesem Rahmen angebotenen Workshops genauer erläutert werden, der im Speziellen das Feedback als Haltung im Spannungsfeld zwischen Beraten und Bewerten in den Blick nimmt. Dazu wird zunächst eine Klärung der grundlegenden Konzepte und Begriffe (Feedback als Haltung, Beraten und Bewerten) vorgenommen. Im Anschluss daran wird die Konzeption des 90-minütigen Workshops vorgestellt und eine Zusammenfassung der erarbeiteten Ergebnisse der Teilnehmenden gegeben. Abschließend wird im Fazit die Relevanz des Feedbacks als Haltung in der Lehrer*innenbildung sichtbar gemacht und es werden Ansätze für die Auflösung des Spannungsfelds zwischen Beraten und Bewerten aufgezeigt.

An dieser Stelle ist hervorzuheben, dass die Autorin dieses Beitrags nicht gleichzeitig die alleinige Urheberin des weiter unten beschriebenen Workshopkonzepts ist. In diesem Zusammenhang sind Maxi Mercedes Grehl und Philipp Nawka zu nennen, die einen wesentlichen Anteil an der inhaltlichen Entwicklung, Planung und Durchführung des Konzepts hatten. Die Wir-Verwendung in diesem Beitrag referiert auf diese Ko-Autorenschaft.

\section{$2 \quad$ Feedback als Haltung}

Feedback im Sinne einer Wirkungsbeschreibung ist eine wesentliche und unverzichtbare Methode im Kontext rhetorischer Didaktik und Methodik (vgl. Geißner/Slembek 1998, zit. nach Meyer 2013, 108). Dabei setzt der konstruk- 
tive und mit einem positiven Lerneffekt verbundene Feedbackprozess eine vertrauensvolle Atmosphäre und einen respektvollen Umgang miteinander voraus. Werden darüber hinaus die im Folgenden näher beschriebenen Regeln beim Geben von Feedback beachtet, kann das Feedback die bewusste Handlungsfreiheit in kommunikativen Situationen steigern (vgl. ebd., 109). Aus einem unabdingbaren konstruktivistisch-systemischen Grundverständnis heraus (vgl. Meyer 2011) ist dabei zu allererst die Subjektivität von Wahrnehmung und die Gebundenheit an die jeweilige Situation zu nennen. Nach Geißner ist es demzufolge beim Feedback unerlässlich, sich der Subjektivität seiner eigenen Wahrnehmung bewusst zu sein und dies im Feedback entsprechend zu formulieren (vgl. Geißner 1998, 17ff.). Aus einer nicht den Feedbackregeln entsprechenden Formulierung wie „Er war hektisch, das hat man gemerkt.“, wird mit deutlich gemachter Subjektivität und darüber hinaus mit einer stets anzustrebenden direkten Anrede der Feedback empfangenden Person die Formulierung „Du hast auf mich auf mich hektisch gewirkt.“

Ferner ist es wichtig, die subjektiv entstandene Wirkung in der Kommunikationssituation durch das Nennen konkreter Beobachtungen zu stützen, damit die Feedback empfangende Person einen Anhaltspunkt hat, welches konkrete Verhalten welche subjektive Wirkung zur Folge hatte; etwa: „Ich habe beobachtet, dass du beim Sprechen hin und her gelaufen bist. Das wirkte hektisch auf mich und ich konnte dadurch nicht gut zuhören." Somit ist das formulierte Feedback nicht nur direkt und subjektiv, sondern darüber hinaus auch konkret und in seiner wertschätzenden Ausdrucksweise damit auch konstruktiv. Diese vier Eckpfeiler des Feedbackgebens lassen sich zwar in Form von Regeln beschreiben (z. B. „Sprich die Person direkt an, formuliere deine Beobachtungen subjektiv, etc.“, vgl. hierzu Meyer 2013, 109ff.); jedoch ist dies allein noch kein Garant für wirklich konstruktives Feedback. Selbst eine an der Oberfläche subjektive Formulierung wie „Ich finde, du warst hektisch“ stellt sich schnell als leere Worthülse heraus, wenn die zugrunde liegende Haltung entsprechend eines konstruktivistisch-systemischen Ansatzes dabei fehlt. Erst durch die Trennung von subjektiver Wahrnehmung (Was habe ich beobachtet?) und sich daraus ergebender subjektiver Reaktion auf diese Wahrnehmung (Wie hat das im Kontext der Situation auf mich gewirkt?) manifestiert sich das anzustrebende Feedback als Haltung, was unserer Auffassung nach tatsächlich 
vielmehr eine Frage der inneren Haltung als das bloße Befolgen von Formulierungsregeln auf oberflächlich-sprachlicher Ebene ist.

Zusammengefasst steht für die Anwendung des Feedback als Haltung im Sinne eines Reflexionsinstruments also immer die Subjektivität und aktuelle Situationsbezogenheit an vorderster Stelle, was gleichzeitig auch bedeutet, sich endgültig von „richtig-falsch“-Kategorien zu verabschieden (vgl. Meyer 2011, 45). Gerade dieser Aspekt führt bei Unterrichtsnachbesprechungen in der Lehrer*innenbildung oft zu Irritationen und Unsicherheiten, da „richtig-falsch“-Denken, Feedback, Beratung und Bewertung hier oftmals nicht reflektiert zum Einsatz kommen. Aus diesem Grund wird im nächsten Abschnitt eine Gegenüberstellung der Begriffe Beraten und Bewerten vorgenommen.

\section{Beraten und Bewerten}

Laut Leitlinie des fächerübergreifenden Erkenntnisgewinns gehören in der Lehrer*innenbildung neben dem Unterrichten, Erziehen und Innovieren auch das Beraten und Bewerten/Beurteilen zu den Kompetenzfeldern in fachwissenschaftlicher, fachdidaktischer und bildungswissenschaftlicher Grundlegung (vgl. Tosch 2018, 344). Mit der Anwendung von Feedback als Haltung in der Lehrer*innenbildung geht oft eine Auseinandersetzung mit diesen Prozessen einher. Wenn ich als Lehrperson oder Mentor*in eine Rückmeldung im Rahmen einer Unterrichtsnachbesprechung gebe - bin ich dann nicht automatisch auch immer in einer Bewertungssituation? Kann in diesem Zusammenhang noch von Feedback gesprochen werden, obwohl sich das Feedback als Haltung explizit nicht als Bewertung, sondern lediglich als Rückmeldung versteht? Hier tut sich ein Spannungsfeld auf. Um dieses sichtbar zu machen, sollen im Folgenden die beiden Prozesse des Beratens und Bewertens näher erläutert werden.

Beratung im Sinne einer pädagogischen Beratung „[...] wird als eine partnerschaftliche Kommunikationsform definiert, in der das professionelle Wissen der Berater und die Lebenserfahrungen und Milieukenntnisse der Ratsuchen- 
den gleichwertig in die Deutungs- und Sinnzuschreibungen einfließen, um so über Reflexionen gemeinsam Lösungen für die sozial typischen Orientierungs- und Entscheidungsproblemlagen moderner Lebensführung zu erarbeiten. " (Tiefel 2012, 110). Für den Prozess des Beratens bedeutet dies, dass es die Aufgabe der ratgebenden Person ist, die Eigenbemühungen und Kompetenzen der ratsuchenden Person zu aktivieren und in den Problemlösungsprozess bzw. die Bewältigung der Situation mit einzubeziehen (vgl. Internetquelle 2).

Bewertung im Sinne einer Leistungsbeurteilung meint dagegen die Kontrolle eines oder mehrerer vorher festgelegter Lernziele hinsichtlich des Erfolgs eines Lernprozesses, der nach individuumsbezogenen, sachbezogenen oder vergleichenden Kriterien erfolgen kann mit dem Zweck, Lerndefizite zu erkennen und ausgleichen zu können, falsche Lernergebnisse zu korrigieren und Lernfortschritte bestätigen zu können (vgl. Reinhold 1999, 350).

Hinsichtlich des Ziels, des Gegenstands sowie der Konstitution des Rollen- und Beziehungsgefüges der an den Prozessen beteiligten Personen können Beraten und Bewerten wie folgt einander gegenüber gestellt werden, vgl. Tab. 1:

\begin{tabular}{|c|c|c|}
\hline Beraten & & Bewerten \\
\hline $\begin{array}{l}\text { Lösen eines Problems der } \\
\text { ratsuchenden Person aus } \\
\text { deren eigener Kraft }\end{array}$ & Ziel & $\begin{array}{l}\text { Fällen eines Urteils über } \\
\text { eine Leistung auf Basis einer } \\
\text { IST-SOLL-Analyse }\end{array}$ \\
\hline $\begin{array}{l}\text { subjektives Empfinden und } \\
\text { Erleben steht im Vorder- } \\
\text { grund }\end{array}$ & Gegenstand & $\begin{array}{l}\text { externe („objektive“) Kriterien } \\
\text { bzw. Maßstäbe werden heran- } \\
\text { gezogen }\end{array}$ \\
\hline Begleiter*in & $\begin{array}{c}\text { Rolle des } \\
\text { Gegenübers }\end{array}$ & Expert*in \\
\hline $\begin{array}{l}\text { setzt voraus, dass die ratsu- } \\
\text { chende Person auch beraten } \\
\text { werden will }\end{array}$ & Beziehungsebene & $\begin{array}{l}\text { es besteht ein Machtgefälle } \\
\text { zwischen bewertender und } \\
\text { bewerteter Person }\end{array}$ \\
\hline $\begin{array}{l}\text { vorrangig Prozessorien- } \\
\text { tierung }\end{array}$ & Fokus & vorrangig Ergebnisorientierung \\
\hline
\end{tabular}

Tab. 1: Gegenüberstellung der Prozesse Beraten und Bewerten 
Diese trennscharfe Gegenüberstellung von Beraten und Bewerten sensibilisiert die Workshopteilnehmer*innen zunächst für die fundamentale Verschiedenheit beider Prozesse, was neben der grundlegenden Erläuterung des Feedbacks als Haltung die theoretische Grundlage für die inhaltliche Arbeit im Workshop darstellt. Im Folgenden werden Konzeption, Ablauf sowie die Ergebnisse des oben genannten Workshops näher beschrieben.

\section{$4 \quad$ Feedback geben im Spannungsfeld zwischen Beraten und Bewerten - Konzeption und Ergebnisse des Workshops}

\section{Zeitrahmen, Zielgruppe und Ziel}

Aufgrund der hohen Nachfrage wurde der 90-minütige Workshop in zwei Durchläufen mit jeweils 20 Personen einmal am Vormittag und einmal am Nachmittag des Workshoptages angeboten. Die Zielgruppe setzte sich in beiden Durchgängen aus Lehrer*innen im Vorbereitungsdienst, Fachdidaktiker*innen der MLU, Haupt- und Fachseminarleiter ${ }^{\star}$ innen des Studienseminars Halle, Mitarbeiter*innen und Studierenden der Sprechwissenschaft sowie aus Mitarbeitenden der Zentren für Lehrerinnenbildung der Universitäten Halle, Leipzig und Potsdam zusammen.

Ziel des Workshops war es, zum einen für das Thema Feedback als Haltung in der Lehrer*innenbildung zu sensibilisieren und zum anderen, den Austausch und die Auseinandersetzung der Akteur*innen untereinander zu Beratungs-, Bewertungs- und Feedbackpraktiken und -prozessen in den verschiedenen Handlungsfeldern anzuregen.

\section{Ablauf}

Nach einer kurzen Orientierungs- und Kennlernphase erhielten die Teilnehmenden die Möglichkeit, mittels eines interaktiven Galerielaufs im Thema anzukommen. Dazu waren an den Wänden und auf den umstehenden Tischen des Raumes Plakate mit Definitionen, Zitaten und teilweise provokanten Thesen rund um die Themen Beraten, Bewerten und Feedback ausgestellt, 
die von den Teilnehmenden schriftlich frei kommentiert, markiert oder auch einfach auch nur zur Kenntnis genommen werden konnten. Bei den meisten der Thesen handelte es sich um authentische Aussagen von Teilnehmer*innen eines Workshops, der drei Monate zuvor im Rahmen des Mentor*innentages Sachsen zu einem ähnlichen Thema stattgefunden hatte. Zur Illustration im Folgenden eine knappe Auswahl dieser Zitate und Thesen:

- "Feedback geht immer mit Beraten und Bewerten einher.“

- „Beratung ist wichtiger als Bewertung.“

- „Die wollen doch bewertet werden!“

- „Eine Leistung die bewertet wird, muss auch wertgeschätzt werden.“

- „Eine Bewertung in Form eines Feedbacks ist ein Wolf im Schafspelz.“

Es folgten daraufhin kurze Theorie-Inputs zu den Themen Beraten vs. Bewerten und Feedback als Haltung wie oben dargestellt. Im Anschluss bekamen die Teilnehmenden die Möglichkeit, in Murmelgruppen mit ihren direkten Sitznachbar*innen zum Impuls „Das Spannungsfeld zwischen Beraten und Bewerten - wie stellt es sich in meinem Arbeitskontext für mich dar?" in einen kurzen Austausch miteinander zu gehen. Im Anschluss daran wurden drei Kleingruppen zu je 5-8 Personen gebildet. Jede Kleingruppe erhielt die Aufgabe, eine oder mehrere der drei im Folgenden genannten Fragen zu bearbeiten und das Gruppenergebnis auf einem Flipchart zu visualisieren. Die drei Fragen lauteten:

a. Wie kann mein Gegenüber in den Beratungs- bzw. Bewertungsprozess mit eingebunden werden?

b. Wie kann ich die Feedback-Haltung subjektiv, konkret, direkt, konstruktiv auf Bewerten und Beraten anwenden?

c. „Die wollen doch bewertet werden. “- Wie steht es um die Bewertungsund Fehlerkultur in meinem Arbeitskontext?

Im Anschluss daran wurden die Gruppenergebnisse im Plenum vorgestellt und abschließend diskutiert. Das Feedback der Teilnehmenden an die Work- 
shopleitung erfolgte in Form der Zielscheibenmethode. Die folgende Tabelle 2 fasst den Ablauf des Workshops überblicksartig zusammen.

\begin{tabular}{|c|c|c|c|}
\hline Zeit & Ziel & Inhalt/Methode & Material \\
\hline & Orientierung & $\begin{array}{l}\text { Begrüßung und Vorstellung der Leitung, } \\
\text { Transparentmachen von Ziel und Ablauf } \\
\text { des Workshops }\end{array}$ & \\
\hline $10^{c}$ & Kennenlernen & soziometrische Aufstellung & \\
\hline $10^{c}$ & $\begin{array}{l}\text { Ankommen im } \\
\text { Thema }\end{array}$ & $\begin{array}{l}\text { Galerielauf } \\
\text { Aufgabe: ausgestellte Zitate und State- } \\
\text { ments zum Thema Feedback/Beraten/ } \\
\text { Bewerten lesen und ggf. kommentieren }\end{array}$ & $\begin{array}{l}\text { vorbereitete Galerie, } \\
\text { Stifte }\end{array}$ \\
\hline $10^{c}$ & $\begin{array}{l}\text { Klarheit über } \\
\text { Begriffe }\end{array}$ & Input zu Beraten vs. Bewerten & Visualisierung \\
\hline $10^{c}$ & $\begin{array}{l}\text { Einführung ins } \\
\text { Feedback }\end{array}$ & Input zum Thema Feedback als Haltung & Visualisierung \\
\hline $10^{c}$ & $\begin{array}{l}\text { Spannungsfeld } \\
\text { sichtbar machen }\end{array}$ & $\begin{array}{l}\text { Murmelgruppen mit Nachbarn } \\
\text { Impuls: „Das Spannungsfeld zwischen } \\
\text { Beraten und Bewerten - wie stellt es sich } \\
\text { in meinem Arbeitskontext für mich dar?“ }\end{array}$ & $\begin{array}{l}\text { Flipchart mit Arbeits- } \\
\text { auftrag }\end{array}$ \\
\hline $15^{c}$ & Transfer & $\begin{array}{l}\text { Aufgabe: eine oder mehrere der vorgege- } \\
\text { benen Fragen in einer Kleingruppe dis- } \\
\text { kutieren und die Ergebnisse auf einem } \\
\text { Flipchart-Blatt festgehalten. Impulse: } \\
\text { Wie kann mein Gegenüber in den } \\
\text { Beratungs- bzw. Bewertungsprozess mit } \\
\text { eingebunden werden? } \\
\text { Wie kann ich die Feedback-Haltung } \\
\text { subjektiv, konkret, direkt, konstruktiv auf } \\
\text { Bewerten und Beraten anwenden? } \\
\text { „Die wollen doch bewertet werden."- Wie } \\
\text { steht es um die Bewertungs- und Fehler- } \\
\text { kultur in meinem Arbeitskontext? }\end{array}$ & $\begin{array}{l}3 \text { verschiedene Bonbon- } \\
\text { sorten zur Gruppenein- } \\
\text { teilung } \\
\text { Flipcharts u. Stifte }\end{array}$ \\
\hline $15^{c}$ & $\begin{array}{l}\text { Präsentation } \\
\text { und Diskussion }\end{array}$ & $\begin{array}{l}\text { Präsentation und Diskussion der Grup- } \\
\text { penergebnisse im Plenum }\end{array}$ & \\
\hline $10^{c}$ & $\begin{array}{l}\text { Feedback an } \\
\text { Workshop-Leite- } \\
\text { rinnen }\end{array}$ & $\begin{array}{l}\text { Zielscheiben-Methode zu den Kategori- } \\
\text { en Inhalt-Methoden-Atmosphäre-Lei- } \\
\text { tung; zusätzlich können Kommentare } \\
\text { dazu schriftlich festgehalten werden }\end{array}$ & $\begin{array}{l}\text { Flipchart, Klebepunkte, } \\
\text { Stifte }\end{array}$ \\
\hline
\end{tabular}

Tab. 2: Planungsmatrix des Workshops 


\section{Ergebnisse}

Im Folgenden wird eine Auswahl der Ergebnisse aus den Gruppenpräsentationen wiedergegeben.

a. Wie kann mein Gegenüber in den Beratungs- bzw. Bewertungsprozess mit eingebunden werden?

- Beraten beinhaltet auch Fragen und Ratsuchende erzählen zu lassen

- Gespräche im Gleichstatus, auf Augenhöhe

- Beratungsprozess transparent machen: Methoden und Techniken erklären, gemeinsam das Ziel festlegen, Fragen stellen

- Mitentscheiden und mitgestalten lassen

b. Wie kann ich die Feedback-Haltung subjektiv, konkret, direkt, konstruktiv auf Bewerten und Beraten anwenden?

- wertschätzende Sprache

- vorhandene Ressourcen nutzen

- notwendige Kompetenzen entwickeln

c. „Die wollen doch bewertet werden." - Wie steht es um die Bewertungs- und Fehlerkultur in meinem Arbeitskontext?

- wir brauchen Kriterien/Parameter

- Beraten ist doch die „Bewertung im Schafspelz“??!

- Transparenz ist wichtig

- Dilemma der Doppelrolle (Mentor*in und Bewerter*in)

In diesem Rahmen ist es nicht möglich, die sich anhand der hier aufgeführten Stichpunkte entspinnenden Diskussionen vollständig und im Detail wiederzugeben. Zusammenfassend wurde in jedem Fall deutlich, dass das eingangs formulierte Ziel des Workshops, nämlich Austausch zu und Auseinandersetzung mit Beratungs-, Bewertungs- und Feedbackpraktiken und -prozessen in verschiedenen Handlungsfeldern, erreicht wurde. Dies bestätigen auch die im Folgenden aufgeführten die Rückmeldungen der Teilnehmenden an die Workshopleitung. 


\section{Rückmeldung der Teilnehmenden zum Workshop}

Für ein abschließendes Feedback der Teilnehmenden an die Workshopleitung wurde die Zielscheiben-Methode verwendet. Dabei erhält jede ${ }^{\star} r$ Teilnehmende vier Klebepunkte, mittels derer angegeben wird, wie sehr der besuchte Workshop hinsichtlich vier verschiedener Kategorien (in diesem Fall theoretischer Input, verwendete Methoden, Atmosphäre und Leitung) für sie oder ihn ,ins Schwarze getroffen" hat. Je dichter die geklebten Punkte am Mittelpunkt sind, desto höher ist die Zufriedenheit.

Insgesamt bildete sich in beiden Durchgängen eine recht hohe Zufriedenheit ab, wobei im zweiten Durchlauf der theoretische Input für die meisten Teilnehmenden nicht ganz ins Schwarze getroffen hatte (s. Abb. 1).

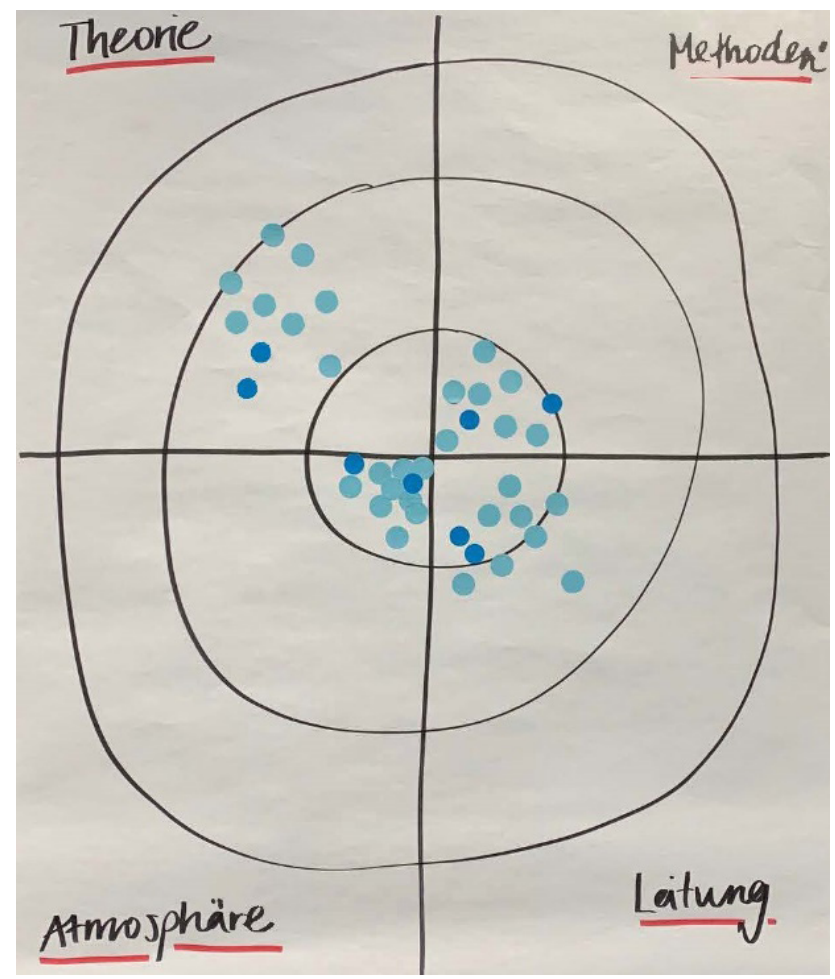

Abb. 1: Zielscheiben-Feedback 
Schriftlich wurde darüber hinaus u. a. Folgendes kommentiert:

- ich nehme mir viel „Gehirnfutter" mit und möchte das Thema weiter vertiefen

- ich möchte Feedback zukünftig stärker in meine Lehre integrieren

- ich nehme mir mit, dass Bewertung kein Feedback ist

- für mich ist Beraten nachhaltiger als Bewerten

Aus Sicht der Workshopleitung können anhand dieser Rückmeldungen, vielmehr aber auf Grundlage der lebendigen Diskussionen in der Auseinandersetzung mit den Fragestellungen im Verlauf, beide Durchgänge des Workshops im Kontext des Workshoptages der „Kleine Fächer-Wochen Sprechwissenschaft“ als gelungen bezeichnet werden.

\section{$5 \quad$ Fazit}

Wie aus den oben aufgeführten Ergebnissen hervorgeht, gibt es einerseits viele Ideen zur konkreten Umsetzung und reflektierten Auseinandersetzung mit Feedback-, Beratungs- und Bewertungsprozessen in den individuellen Arbeitsfeldern. Dennoch spiegeln einige der Beiträge („Beraten ist doch die Bewertung im Schafspelz??!") auch eine Ratlosigkeit wider, wie das Spannungsfeld zwischen Beraten und Bewerten konkret aufgelöst werden könnte und welche Rolle das Feedback in diesen Prozessen spielt. Aus diesem Grund schlagen wir im Folgenden ein Schema vor, inwiefern das Feedback als Haltung in beiden Prozessen greifen und zu einem konstruktiven Miteinander beitragen kann.

Wie oben in Abschnitt 2 beschrieben, wird aus der möglichst konkreten Beschreibung einer subjektiven Wahrnehmung („Ich habe beobachtet, dass du beim Sprechen hin und her gelaufen bist.") und der sich anschließenden Beschreibung einer subjektiven Wirkung („Das wirkte hektisch auf mich und ich konnte dadurch nicht gut zuhören.") in einer direkten, wertschätzenden Formulierung an die Feedback empfangende Person ein konstruktive Rückmeldung, die dem Feedback als Haltung entspricht und somit die bewusste 
Handlungsfreiheit in kommunikativen Situationen steigern und zu einem positiven Selbstwirksamkeitserleben führen kann.

In diesem Zusammenhang hat Annelies Kreis (vgl. 2012, 274) nachgewiesen, dass bei der Reflexion über das eigene Verhalten ein höherer Lerneffekt erzielt wird, wenn es nicht in monologisch strukturierten Gesprächen, sondern in einem ko-konstruktiven Prozess stattfindet. Das heißt, idealerweise schließt sich an das Feedback eine Frage an („Wie ging es dir selber in dieser Situation?"), die das Gegenüber dazu einlädt, in einen dialogischen Austausch einzutreten. Dazu sind offene und systemische Fragen am besten geeignet (vgl. Pfeiffer 2019, 63). Wie in Abb. 2 dargestellt, ergibt sich aus der Abfolge von Feedback (bestehend aus konkreter Beschreibung der subjektiven Wahrnehmung und deren subjektiver Wirkung) und sich anschließender Frage der Impuls zu einem ko-konstruktiven Miteinander im Gespräch. Gelingt den Interaktant ${ }^{\star}$ innen dabei, wie oben für das Format Beratung definiert, eine partnerschaftliche, dialogische Kommunikationsform, ist der Grundstein für die Gestaltung eines positiven Reflexionsprozesses gelegt.

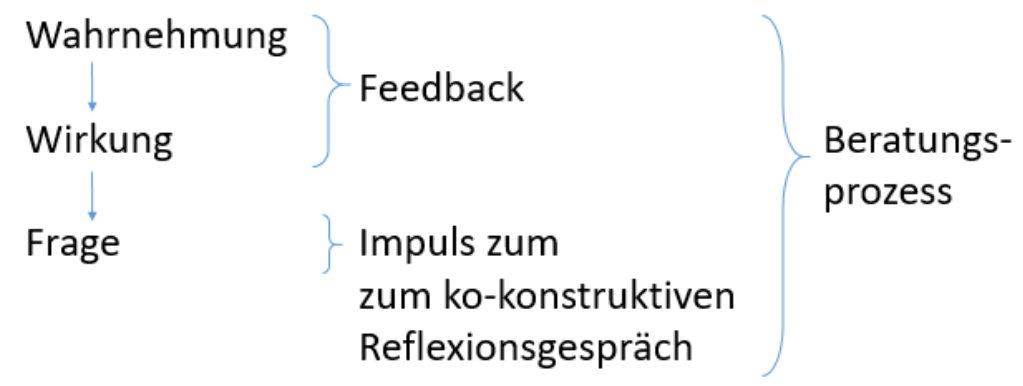

Abb. 2: Feedbackgeben im Rahmen eines Beratungsprozesses

Im Vergleich dazu wäre je nach Situation und Kontext auch denkbar, dass sich an die Beschreibung der subjektiven Wahrnehmung und Wirkung im Feedback ein Urteil bzw. eine Leistungsbewertung anschließt („Aus diesem Grund hast du für mich in der Kategorie Methodenvielfalt nur acht von zehn Punkten erreicht.“), vgl. Abb. 3. Damit kann das Feedback auch Teil eines Bewertungsprozesses sein, der durch die Offenlegung der einfließenden Kategorien transparent gemacht 
wird und somit ebenfalls zur Perspektiverweiterung und Kompetenzentwicklung der angehenden Lehrkraft beitragen kann (vgl. Meyer 2018, 227).

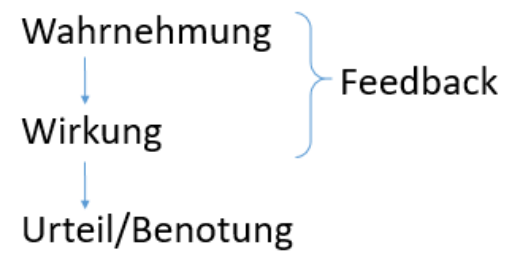

transparent gemachter Bewertungsprozess

Abb. 3: Feedbackgeben im Rahmen eines Bewertungsprozesses

Abschließend möchten wir, die Ko-Autorenschaft des Workshops, betonen, dass der mit einem positiven Lerneffekt verbundene Feedbackprozess eine achtsam und respektvoll gestaltete Beziehung gleichermaßen voraussetzt wie auch aktiv mitgestaltet. Feedback als Haltung ist in der Lehrerinnenbildung demzufolge von höchster Relevanz. Denn: „So, wie wir heute miteinander sprechen, werden wir morgen miteinander leben." (Voigt-Zimmermann 2019, Initiatorin der „Kleine Fächer-Woche Sprechwissenschaft“ an der Martin-Luther-Universität Halle-Wittenberg).

\section{Literatur}

Behnke, Kristin (2016): Umgang mit Feedback im Kontext Schule. Erkenntnisse aus Analysen der externen Evaluation und des Referendariats. Wiesbaden: Springer. DOI 10.1007/978-3-658-10223-4_10

Geißner, Hellmut (1998): Vom Oberflächen- zum Tiefenfeedback. In: Geißner, Hellmut/Slembek, Edith (Hg.): Feedback: Das Selbstbild im Spiegel der Fremdbilder. St. Ingbert: Röhrig. S. 13-30.

Geißner, Hellmut/Slembek, Edith (1998) (Hg.): Feedback: Das Selbstbild im Spiegel der Fremdbilder. St. Ingbert: Röhrig.

Kreis, Annelies (2012): Produktive Unterrichtsbesprechungen. Lernen im Dialog zwischen Mentoren und angehenden Lehrpersonen. Bern: Haupt. 
Meyer, Dirk (2011): Alles nur Vermutung? Gedanken zur interpersonellen Kommunikation aus konstruktivistisch-systemischer Sicht. In: Bose, Ines/Neuber, Baldur (Hg.): Interpersonelle Kommunikation: Analyse und Optimierung. (=HSSP 39). Frankfurt a. M.: Peter Lang, S. 201-207.

Meyer, Dirk (2013): Analyse rhetorischer Ereignisse. Beobachtung, Feedback, Evaluation. In: Bose, Ines/Hirschfeld, Ursula/Neuber, Baldur/Stock, Eberhard (2013): Einführung in die Sprechwissenschaft. Phonetik, Rhetorik, Sprechkunst. Tübingen: Narr. S. 108-112.

Meyer, Hilbert (2018): Leitfaden Unterrichtsvorbereitung. Berlin: Cornelsen.

Pfeiffer, Alexander (2019): Professionalisierung im Lehramtsstudium durch videogestützte Fallarbeit in den Praxisphasen. In: Klektau, Claudia et al. (Hg.): Heterogenitätssensibilität durch Fallarbeit fördern. Zum Stellenwert von Kasuistik und Inklusion in der Lehrer*innenbildung. (Hallesche Beiträge zur Lehrer*innenbildung, 1). S. 59-66. urn:nbn:de:gbv:3:2-106991

Reinhold, Gerd (1999) (Hg.): Pädagogik-Lexikon. München, Wien: Oldenbourg Verlag. DOI: 10.1515/9783486785227

Schledde, Walter (2007): Empfehlungen zur (Fach-) Seminargestaltung. In: Ansorge, Olaf et al. (Hg.): (Beratungs-)Gespräche in der Lehrerbildung - Prinzipien und Verfahren für eine größere Glaubwürdigkeit. Hannover: Gewerkschaft Erziehung und Wissenschaft (GEW), Landesverband Niedersachsen. S. 13-16. https://uol.de/fileadmin/user_upload/diz/ofz/pdf/GEWBeratungsgespraeche.pdf Tiefel, Sandra (2012): Beratung. In: Horn, Klaus-Peter et al. (Hg.): Klinkhardt Lexikon Erziehungswissenschaft. Bad Heilbrunn: Verlag Julius Klinkhardt. S. $109 f$.

Tosch, Frank (2018): Lehrerbildung und Schulpraktische Studien im Spiegel eines Spiralcurriculums. Vernetzungsperspektiven im Potsdamer Modell. In: Potsdamer Beiträge zur Lehrerbildung und Bildungsforschung. S. 335- 348. https://publishup.uni-potsdam.de/opus4-ubp/frontdoor/deliver/index/ docId/42044/file/pblbf01_335-348.pdf

Voigt-Zimmermann, Susanne (2019): Kleine Fächer-Woche Sprechwissenschaft, Thema „miteinander sprechen - verantwortlich, kompetent, reflektiert“. Projektskizze zum Antrag auf Finanzierung durch die Hochschulrektorenkonferenz vom 12.02.2019

\section{Internetquellen}

Internetquelle 1: https://www.qualitaetsoffensive-lehrerbildung.de/de/feedbackkulturin-der-lehrer-innenbildung---ein-praxisorientierter-workshop-tag-in-halle2264.html (Letzter Zugriff 24.02.2021)

Internetquelle 2: https://lexikon. stangl.eu/2846/beratung/ (Letzter Zugriff 24.02.2021) 

Ines Bose/Stephanie Kurtenbach / Franziska Kreutzer

und Kerstin Kreße, Halle (SaAle)

\title{
Mit Kindern im Gespräch. Sprechwissenschaftliche Kita-Forschung mit Praxiskooperationen
}

Zusammenfassung: Ein Forschungsschwerpunkt der Abteilung Sprechwissenschaft in Halle ist das Gesprächsverhalten ganz junger Menschen. Wie Kinder miteinander ins Gespräch kommen, aber auch wie pädagogische Fachkräfte mit Kindern sprechen, untersuchen wir seit 13 Jahren in verschiedenen Projekten und mit unterschiedlichen Praxispartnern. Im Beitrag werden diese Wissenschafts-Praxiskooperationen vorgestellt. Wesentliche Erkenntnisse aus den Untersuchungen zum Gesprächsverhalten werden jeweils abgeleitet. Diese liefern wertvolle Handlungsorientierungen für die Weiterentwicklung der Projekte und für die Fortbildung von pädagogischen Fachkräften liefern.

Stichwort: Kindergarten, Sprachförderung, Fachkraft-Kind-Interaktionen, Gespräche

\begin{abstract}
A research focus of the Speech Science department in Halle is the conversational behavior of very young people. For 13 years, we have been investigating how children get into conversation with one another, but also how educational professionals talk to children, in various projects and with different kindergarten practice partners. These scientific-practice collaborations are presented in the paper. Essential findings from the investigations into conversation behavior are derived in each case. These provide valuable orientations for the further development of the projects and for the advanced training of educational specialists.
\end{abstract}

Keywords: daycare, language training, kindergarten teacher-child interactions, discussions 
Wie Menschen miteinander reden, beschäftigt unser Fach in Lehre Forschung und Praxis von Beginn an. Ein aktuell wachsender Forschungsschwerpunkt in Halle ist das Gesprächsverhalten ganz junger Menschen. Wie Kinder miteinander ins Gespräch kommen, aber auch wie pädagogische Fachkräfte mit Kindern sprechen, untersuchen wir in verschiedenen Projekten und mit unterschiedlichen Praxispartner ${ }^{\star}$ innen. Im folgenden Beitrag möchten wir ein Resümee über die letzten dreizehn Jahre ziehen, in denen wir gemeinsam mit diesen Partner*innen Forschungsprojekte entwickeln, umsetzen und evaluieren konnten. Wir möchten aus diesen Projekten vor allem Erkenntnisse vorstellen, die aus den Untersuchungen zum Gesprächsverhalten von Kindern und ihren pädagogischen Fachkräften gewonnen wurden und uns wertvolle Handlungsorientierungen für die Weiterentwicklung der Projekte und für die Fortbildung von pädagogischen Fachkräften liefern können. Wir gehen bei der Vorstellung der Projekte chronologisch vor, so dass dies auch als zeitlicher Überblick der einzelnen Projekte betrachtet werden kann.

\section{Projekt „Bilderbuchbetrachtungen mit Kita-Kindern“}

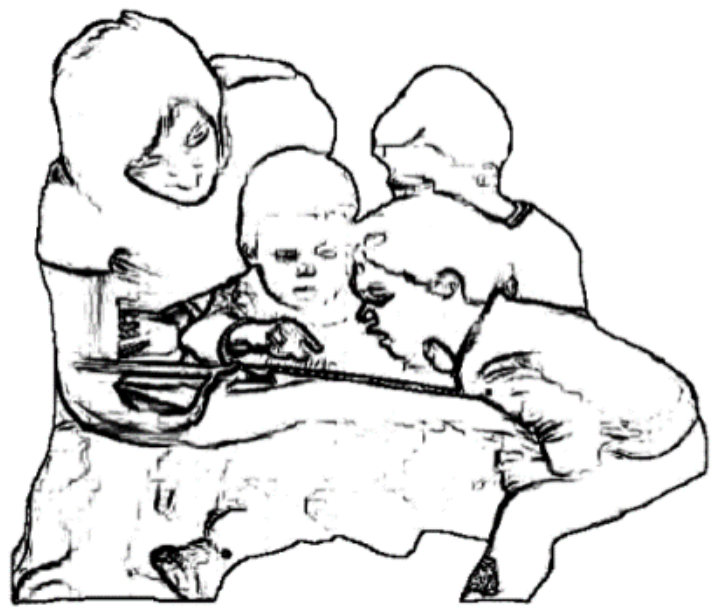

Abb. 1: Bilderbuchbetrachtung mit zweijährigen Kindern in der Kita 
Nahezu täglich begeben sich pädagogische Fachkräfte mit Kindern in eine gemütliche Ecke des Kindergartens und sehen gemeinsam Bilderbücher an. Diese Form des kommunikativen Miteinanders ist eine ganz besondere, da sie vielerlei Möglichkeiten bietet, Kinder in ihrer Sprachentwicklung zu unterstützen und zu fördern. Im Landesmodellprojekt „Sprache fördern - Erprobung und Multiplikation von Methoden der Sprachförderung " des Freistaates Sachsen (Abschlussbericht des BBW 2011) bildete neben einer alltagsintegrierten Sprachbildung das regelmäßige gemeinsame Anschauen von Bilderbüchern mit Kindern einen wesentlichen Bestandteil der Förderung. Die Sprechwissenschaft war an der Konzeption dieses Projekts wie auch an seiner Evaluierung beteiligt (Kurtenbach 2011). Um Schulungseffekte zu untersuchen, wurden diese Bilderbuchbetrachtungen vor und nach der Schulungsphase (2009 und 2010) im Rahmen einer Qualifizierungsarbeit videografiert (Kurtenbach/Bose/ Thieme 2013). Es entstand ein Korpus von 44 Aufnahmen: Elf pädagogische Fachkräfte wurden mit jeweils zwei Kindergruppen unterschiedlichen Alters (Zweijährige und Vierjährige) gefilmt. Die Aufnahmen wurden am Beginn und am Ende der Schulungsmaßnahme durchgeführt. Beim Auswerten des Datenmaterials haben wir die folgenden Forschungsfragen verfolgt:

- Welche sprach- und kommunikationsförderlichen Strategien werden von den pädagogischen Fachkräften in den Bilderbuchbetrachtungen eingesetzt?

- Inwieweit beeinflussen diese Strategien das sprachliche Verhalten der Kinder im konkreten Kontext?

Das Gesprächsverhalten aller Beteiligten (Kinder und Fachkräfte) wurde in einem Mixed-Method-Design analysiert: Der Einsatz der Strategien wurde anhand der jeweils ersten 100 Äußerungen der Fachkräfte erhoben und quantitativ ausgewertet (vgl. Kupietz 2013). Darüber hinaus analysierten wir in ausgewählten Gesprächspassagen den interaktiven Umgang mit Sprachlehrstrategien qualitativ-deskriptiv. Hier sind wir der linguistischen Gesprächsforschung konversationsanalytischer Prägung gefolgt (vgl. stellvertretend Deppermann 2008). Mit diesem Methodendesign konnten wir wichtige Erkenntnisse darüber gewinnen, welche Wirkung die bisher als sprachförderlich geltenden 
Strategien tatsächlich in konkreten Gesprächsprozessen haben (vgl. Bose et al. 2013; Kurtenbach et al. 2013; Bose/Kurtenbach 2014). Im vorliegenden Beitrag konzentrieren wir uns auf die folgenden Sprachlehrstrategien: Wiederholungen kindlicher Äußerungen, thematische Fortführungen (Extensionen) und Fragehandlungen (ausführlicher Kurtenbach et al. 2013; Kurtenbach/Bose 2014).

Wiederholungen kindlicher Äußerungen gelten als sprachförderlich, weil sie das gegenseitige Verständnis sichern und den Kindern die Verarbeitung der produzierten Äußerung ermöglichen. Die qualitativen Analysen zeigen, dass Wiederholungen die kindliche Erzählfreude vor allem dann anregen, wenn die Fachkräfte das Thema der Kinder im Anschluss an die Wiederholung erweitern oder fortführen. Bloße Wiederholungen kindlicher Äußerungen ohne Verortung im Kontext wirken sich im Korpus negativ auf die Gesprächshaftigkeit aus: Das Gespräch verbleibt dann im Modus des Benennens von Bilddetails, ohne dass ein gemeinsames Thema bearbeitet wird. Die verbesserte Wiederholung (engl. corrective feedback, vgl. Kannengieser 2019) ist eine Sonderform des Wiederholens und gilt als besondere sprachförderliche Strategie: Fehlerhafte Äußerungen der Kinder werden in einer korrekten Form wiederholt, ohne auf den jeweiligen Fehler hinzuweisen. So haben die Kinder die Möglichkeit, eine korrigierte Version ihrer Äußerung zu hören, aber ihre Äußerungen werden nicht kritisiert.

Die verbesserte Wiederholung ist die im Korpus am seltensten eingesetzte Sprachlehrstrategie. Dies ist insofern verwunderlich, als dass diese Strategie in der Sprachförderliteratur als sehr wichtig und auch wirkungsvoll für den Sprachlernprozess der Kinder angesehen wird (vgl. Möller 2006; Manolson 1992). In den seltenen Fällen, in denen diese Strategie im Korpus eingesetzt wird, folgt häufig eine Erweiterung der kindlichen Äußerung, wie der folgende Transkriptausschnitt zeigt (Legende der Notationszeichen am Ende des Beitrags):

\begin{tabular}{|c|c|c|}
\hline 030 & PFk : & $\begin{array}{l}\text { und was sehn wa=n } \uparrow \text { NOCH 'hier vorne auf dem } \\
\text { buch.।I }\end{array}$ \\
\hline 031 & & GUCK=ma mal. || \\
\hline 032 & KATI : & $\uparrow v e: \downarrow g e l .||$ \\
\hline 033 & PFk : & 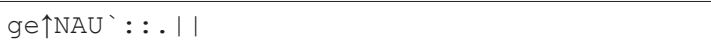 \\
\hline 034 & & die $\uparrow$ VöGllein sind hier auf dem buch.|| \\
\hline
\end{tabular}

Beispiel 1: Bilderbuchbetrachtung in der Kita - LM019_01.03; Zweijährige 
Drei zweijährige Kinder und die pädagogische Fachkraft betrachten das Titelbild eines Bilderbuches. Die Fachkraft unterhält sich mit den Kindern darüber, was auf dem Titelbild zu sehen ist. Sie bestätigt zunächst Katis Äußerung $\uparrow v e: \downarrow g e l .||(Z .32)$ mit $g e \uparrow N A U^{\prime}:: .||$ (Z. 33) und wiederholt dann die kindliche Äußerung in einer korrekten Form, eingebettet in eine Äußerung (Z. 34) (ausführlicher vgl. Kurtenbach et al. 2013, 34ff.).

Thematische Fortführungen kindlicher Äußerungen durch die Fachkräfte sollen eine hohe kommunikationsförderliche Wirkung haben. Dies zeigt sich auch in unseren Daten. Die Kinder konzentrieren sich häufig auf einen thematischen Fokus und bleiben mitunter über mehrere Turns gemeinsam bei einem etablierten Thema. Nach Angabe vieler Sprachfördermaßnahmen (vgl. Ruberg/ Rothweiler 2012; Siegmüller/Frohling 2010; Seidl 2008) gelten bestimmte Fragetypen im Kontakt mit Kindern als sprachförderlich (vor allem offene Frageformen) und andere eher als gesprächshemmend. Diese Annahme wollten wir in unseren Daten prüfen. Hierfür wurden zehn Gespräche ausgewertet, welche zwei Gesprächstypen zugeordnet wurden. Fünf Gespräche (Typ A) zeichnen sich als intensiv gesprächshaft aus: Fachkräfte und Kinder entwickeln über mehrere Turns gemeinsame Themen. Außerdem wurden fünf weitere Gespräche (Typ B) ausgewählt, die solche gesprächshaften Passagen nicht aufweisen. In letzteren fällt auf, dass die Fachkräfte häufig Testfragen zur Strukturierung nutzen („Wo seht ihr...?“, „Was ist ...?"), worauf die Kinder mit dem Benennen von Detailbildern antworten.

Beide Teilkorpora weisen eine hohe, fast identische Anzahl von Fragehandlungen auf: 226 bzw. 227 (bezogen auf jeweils 500 Äußerungen der pädagogischen Fachkräfte). Alternativfragen kommen kaum vor (je zweimal), Entscheidungs- und Ergänzungsfragen sind weitaus häufiger. In den intensiven Gesprächen sind Entscheidungsfragen (z. B. „Hast du einen LKW gesehen?“” „Habt ihr auch schonmal sowas erlebt?“, „Wart ihr auch schonmal auf einem Bahnhof?") der häufigste Fragetyp. Im weniger gesprächshaften (nicht themenzentrierten) Teilkorpus dagegen finden sich etwas mehr Ergänzungsals Entscheidungsfragen (55\% vs. $44 \%$ ), z. B. „Was siehst du noch auf dem Bild?“, „Wo steht das Müllauto?“. Die qualitativen Analysen zeigen, dass für die sprachanregende Wirkung von Fragen nicht der Fragetyp entscheidend 
ist, sondern der konkrete Gesprächskontext, aus dem die Fragen entstehen (vgl. auch Kurtenbach 2009; Arnold et al. zit. nach Ruberg/Rothweiler 2012).

Alle unsere Analysen zu diesem Videokorpus von Bilderbuchbetrachtungen zeigen, dass sich die Sprachlehrstrategien immer nur dann als sprach- und gesprächsförderlich erweisen, wenn sie in einen für die Kinder bedeutungsvollen Kontext eingebettet sind und sich an ihrem aktuellen Interesse und ihren Erfahrungen orientieren. Dann können die Kinder zu eigenen Äußerungsproduktionen angeregt werden und auch sehr junge Kinder können ein etabliertes Thema mitunter über mehrere Turns verfolgen. In unseren Weiterbildungen sensibilisieren wir daher pädagogische Fachkräfte vor allem für das eigene Gesprächsverhalten und das der Kinder - und zwar in konkreten Gesprächskontexten (per Videoreflektion). Im Betrachten konkreter Gesprächssituationen können Alternativen zum aktuellen Gesprächsverhalten entwickelt und dann im Kitaalltag ausprobiert werden.

\section{Projekt: „Kinderleicht sprechen...“}

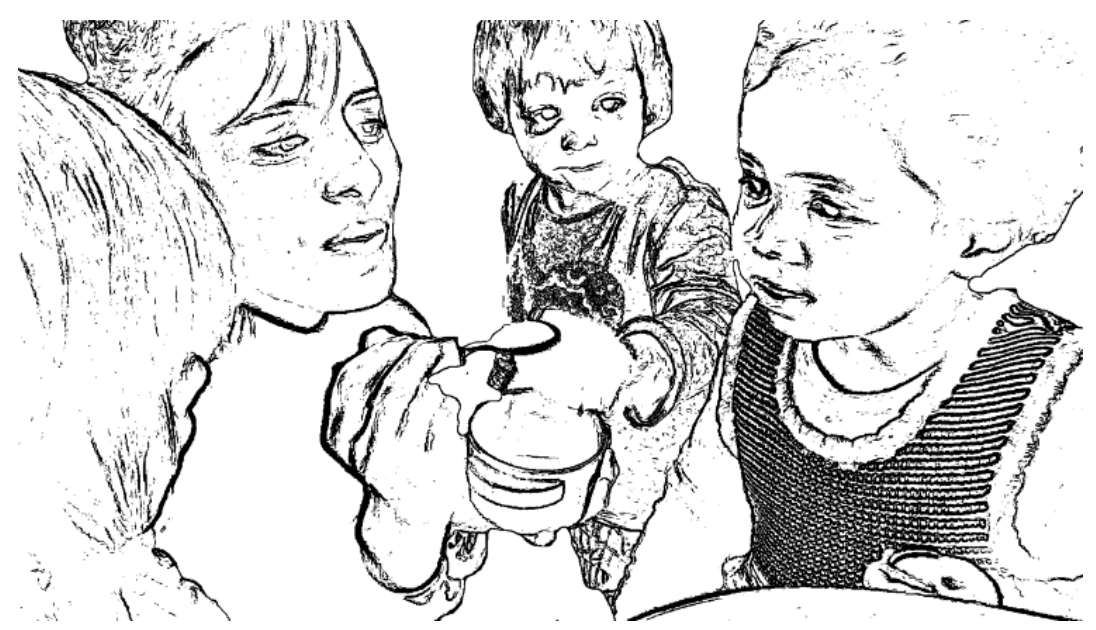

Abb. 2: Spiel „Kochen und Kosten“ im Projekt „Kinderleicht sprechen...“ 
Seit 2010 führt die Sprechwissenschaft an der Martin-Luther-Universität HalleWittenberg gemeinsam mit dem Eigenbetrieb Kindertagesstätten jährlich das Weiterbildungsprojekt „Kinderleicht sprechen...“ durch. Ziel des Projektes ist die nachhaltige Umsetzung einer feinfühligen Interaktion und Kommunikation zwischen pädagogischen Fachkräften und Kindern, um sowohl präventiv als auch operativ die Sprachentwicklung zu begleiten und zu fördern. Personell wird das Projekt getragen von dem Abschlussjahr des jeweiligen Masterstudienganges Sprechwissenschaft und den teilnehmenden pädagogischen Fachkräften des Eigenbetriebes Kindertagesstätten Halle und freier Träger. Die Qualifizierungen werden jährlich evaluiert, um Inhalte kontinuierlich praxisorientiert zu reflektieren und zu optimieren. Was 2010 noch als zweitägige Weiterbildung mit Videodokumentation und -auswertung begann, ist heute ein Tandemprojekt, das über einen Zeitraum von sechs Monaten durchgeführt wird: Die Studierenden lernen und arbeiten gleichberechtigt gemeinsam mit den Fachkräften im Tandem an vier Weiterbildungstagen und drei Praxistagen im Wechsel. Im Mittelpunkt steht dabei die Videodokumentation: Sie dient der Beobachtung der Kinder auf Grundlage der projekteigenen Beobachtungsbögen (vgl. Kurtenbach et al. 2011). Darüber hinaus fördert sie die eigene Reflexion als Ausgangspunkt für Veränderung und Optimierung der kommunikativen Kompetenz.

Bisher wurden verschiedene thematische Schwerpunkte gesetzt: 2010 startete das Projekt mit der Sprachbildung und -förderung der Kinder von 0-6 Jahren. Bereits im zweiten Projektjahr wurde eine thematische Fokussierung vorgenommen: Das Alter der Kinder wurde auf 0-2 Jahre eingegrenzt, um der wichtigen Phase der vorsprachlichen Entwicklung mehr Raum zu geben. Das Projekt bekam den Titel „Kinderleicht sprechen von Anfang an - Fachkräfte und Studierende im Tandem“. Der Fokus der Schulungsinhalte lag darauf, die pädagogischen Kräfte in der Beobachtung und Begleitung kommunikativer Vorausläuferfähigkeiten der Kinder zu schulen. Aufgrund der hohen Nachfrage der Kitas wurde dieses Thema insgesamt fünfmal in fünf Projektjahren angeboten. Seit 2018 wird das Projekt mit einem neuen Thema weitergeführt: "Kinderleicht sprechen ... quersprachig - mehrsprachig". Im Mittelpunkt der Schulungen und Praxisaufträge stehen nun die Sprachentwicklung mehrspra- 
chiger Kinder sowie die Chancen und Herausforderungen gelebter Mehrsprachigkeit im Kita-Alltag.

Das Projekt „Kinderleicht sprechen ..." wurde von Beginn an wissenschaftlich begleitet. Diese Begleitforschung dient mehreren Zwecken. Einen Forschungsschwerpunkt stellt die Analyse der Kommunikationssituationen wie auch des Interaktionsverhaltens von Kindern und pädagogischen Fachkräften dar (Harz 2016, Schäuble 2020). Konkrete Forschungsfragen können so durch das Projekt gestellt und in Form von Qualifizierungsarbeiten untersucht werden. Diese Auftragsarbeiten verfolgen das Ziel, Gelingensbedingungen erfolgreicher Kommunikationssituationen herauszufiltern und in das Schulungsprojekt als best-practise-Beispiele zu integrieren. So konnte z. B. Schäuble (2020) mittels einer Videoanalyse herausarbeiten, dass in der Kommunikation mit mehrsprachig aufwachsenden Kindern neben bereits bekannten Sprachförderstrategien (verbesserte Wiederholung, handlungsbegleitendes Sprechen, thematische Fortführungen, Fragehandlungen) die pädagogischen Fachkräfte weitere Verhaltensweisen erfolgreich einsetzen, um die Kinder zur Kommunikation anregen. Hierzu gehören eine intensive Bindung zu den Kindern, eine sensible Moderation von Peer-Interaktionen, Geduld und Abwarten beim Zuhören, ein behutsamer Umgang mit Fragen, Flexibilität und Spontaneität im Kontakt mit den Kindern, Spaß und gemeinsames Lachen wie auch die Verknüpfung von Sprache und Bewegung. Durch datenbasierte Untersuchungen wie diese können nicht nur Gelingensbedingungen für Fachkraft-Kind-Interaktionen unter die Lupe genommen, sondern auch Schulungsinhalte des Projektes „Kinderleicht sprechen“ reflektiert und kontinuierlich optimiert werden. Außerdem können so Schulungseffekte evaluiert werden.

Innerhalb der zehnjährigen Projektlaufzeit sind projekteigene Schulungsmaterialien erstellt und evaluiert worden. Eines davon ist der Beo, der „Beobachtungsbogen für frühe kommunikative Fähigkeiten“, mit dem kommunikative Vorausläuferfähigkeiten bei Kindern beobachtet werden können. Darüber hinaus bietet der Bogen die Möglichkeit, Handlungsideen zur Anbahnung und Förderung kommunikativer Fähigkeiten bei Kindern abzuleiten. Der Bogen wurde durch teilnehmende Studentinnen des Projektes in wissenschaftlichen Abschlussarbeiten evaluiert. Mittlerweile liegt mit dem Beo ein qualifiziertes Beobachtungsinstrument vor, das die Gütekriterien Objektivität, Reliabilität 
und Praktikabilität erfüllt (Kurtenbach et al. 2019). Er steht mit seinem Begleitheft auf den Internetseiten des Eigenbetriebes Kindertagesstätten zur freien Verfügung (Kurtenbach et al. 2011).

\section{Gesprächskreisprojekt „Stolpersteine \& Wunschsterne“}

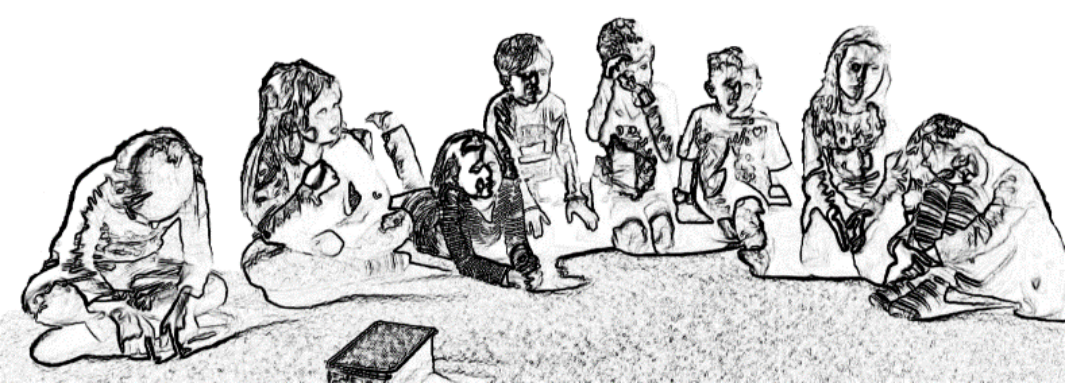

Abb. 3: Kita-Gesprächskreis „Stolpersteine \& Wunschsterne“

Seit 2014 finden in der Kindertagesstätte „Am Moritzburgring" freiwillige Gesprächskreise statt, in denen fünf- bis sechsjährige Kinder gesellschaftliche Partizipation intensiv erleben und ausprobieren. Regelmäßig besprechen sie relevante Themen der Kita, treffen gemeinsame Entscheidungen und erproben sie. Im Rahmen einer Wissenschafts-Praxis-Kooperation haben wir diese Gesprächskreise über mehrere Perioden hinweg videografiert (34 Gesprächskreise, Gesamtumfang ca. 8,5 h) (vgl. Bose/Kurtenbach 2019). Das Projekt ist für uns deshalb besonders interessant, weil wir aus der Fachliteratur und eigenen Untersuchungen wissen, dass Gesprächskreise zwar in Kitas beliebt sind und als Sprachförderanlass gelten (Kurtenbach et al. 2013), dass sie aber oft gekennzeichnet sind durch große Dominanz der Fachkräfte, da diese die Themen setzen, das Rederecht regeln und selbst hohe Redeanteile aufweisen (ebd.). Im Gesprächskreisprojekt „Stolpersteine \& Wunschsterne“ verhandeln die Kinder jedoch ausschließlich eigene Themen. Das sind meist Kitaange- 
legenheiten (z. B. Ausflugswünsche oder Konflikte unter den Kindern). Wir wollten wissen, welche Auswirkungen dies auf den Gesprächscharakter hat.

Wir haben im Gesprächsverlauf nach typischen Verlaufsstrukturen des Formats sowie nach Mustern im Gesprächshandeln von Fachkräften und Kindern gesucht, z. B. nach Formen kindlicher Argumentation und Kooperation, formalsprachlichen Kennzeichen und gesprächsförderlichem Handeln der Fachkräfte (Bose/Kurtenbach 2019; Bose/Hannken-Illjes/Kurtenbach 2020). So konnten wir anhand der Aufnahmen und der Gesprächstranskripte erkennen, dass sich in dem Projekt bei der Bearbeitung der kindlichen Themen intuitiv eine typische Verlaufsstruktur etabliert hat und dass sich im Umgang mit kindlichen Anliegen wiederkehrende Gesprächspraktiken finden lassen (ausführlicher Bose/Kurtenbach 2019).

\begin{tabular}{|l|l|}
\hline Verlaufsstruktur & Praktiken \\
\hline 1. Themenkategorisierung & $\begin{array}{l}\text { Die Kinder benennen ihre Anliegen und kategorisieren sie als } \\
\text { „Stolperstein“ (Problem) oder „Wunschstern“ (Wunsch). }\end{array}$ \\
\hline 2. Themenspezifizierung & $\begin{array}{l}\text { Mithilfe der pädagogischen Fachkräfte werden die Themen } \\
\text { ausformuliert und konkretisiert, damit sie für alle Kinder } \\
\text { verständlich werden. }\end{array}$ \\
\hline 3. Themenbearbeitung & $\begin{array}{l}\text { Lösungsvorschläge der Kinder werden gesammelt und hin- } \\
\text { sichtlich der Tauglichkeit gegeneinander abgewogen. }\end{array}$ \\
\hline 4. Entscheidung & $\begin{array}{l}\text { Die Gesprächsgruppe entscheidet sich für die momentan } \\
\text { sinnvollste Lösung. Die Fachkräfte sorgen für eine zeitnahe } \\
\text { Umsetzung. }\end{array}$ \\
\hline
\end{tabular}

Tabelle 1: Verlaufsstruktur und Gesprächspraktiken „Stolpersteine \& Wunschsterne“

Zunächst wählen die Kinder ein Symbol und kategorisieren damit ihr Anliegen als „Stolperstein“ (Problem) oder „Wunschstern“ (Wunsch) (Themenkategorisierung). Die Videoaufnahmen zeigen, dass es für die Kinder durchaus anspruchsvoll ist, zu entscheiden, ob sie ein Anliegen vorbringen wollen und wenn ja, ob das eher ein Wunsch oder ein Problem ist. Oft helfen die Fachkräfte (und andere Kinder) bei der Kategorisierung und auch dabei, die Themen auszuformulieren und konkreter zu beschreiben, damit sie für alle Kinder verständlich werden (Themenformulierung und-spezifizierung). Dafür führen wir ein Beispiel an (Beispiel 2, Teil 1): 


\begin{tabular}{|c|c|c|}
\hline 01 & PFk : & $\begin{array}{l}\text { pauLIne was hattest DU denn für einen } \\
\text { STOLperstein.|| }\end{array}$ \\
\hline 02 & Pauline: & ähm ähm ich und JUlia und SANdra, I \\
\hline 03 & & $\begin{array}{l}\text { (.) da hatten wir_n TUCH und DAS(.) lag daHINten(.) } \\
\text { zer-KNOtet in der ECke.|| }\end{array}$ \\
\hline 04 & PFk : & (.) und WAS hat dir daran jetzt nicht geFALlen?| \\
\hline 05 & & was was ist da was STÖRT dich da?|| \\
\hline 06 & Pauline: & da mussten wir dann ALle knoten RAUSmachen;।| \\
\hline 07 & PFk : & dass das zerkNotet war; || \\
\hline 08 & Pauline: & $((n i c k t))$ \\
\hline 09 & PFk : & $\begin{array}{l}\text { und du MÖCHtest nicht dass die TÜcher zerkNOtet } \\
\text { sind?|| }\end{array}$ \\
\hline 10 & Pauline: & ((schüttelt den Kopf)) \\
\hline
\end{tabular}

Beispiel 2: Gesprächskreis 1 (00:01:12): Paulines Stolperstein „Zerknotete Tücher“ (Teil 1)

Pauline hat einen Stolperstein in der Hand und berichtet, dass ein Spieltuch zerknotet in der Ecke gelegen hat (Z.02). Die Fachkraft sorgt mit Nachfragen (Z. 04-05) und Paraphrasierungen (Z. 07 und 09) dafür, dass Paulines Anliegen als Problem spezifiziert wird: Pauline und ihre Freundinnen mussten die Knoten erst lösen, bevor sie mit den Tüchern spielen konnten (Z. 06), und das möchte Pauline nicht mehr (Z.09-10).

Bei der Themenformulierung und -spezifizierung spielt die Gesprächsmoderation der pädagogischen Fachkräfte eine große Rolle: Sie unterstützen das jeweilige Kind, indem sie nachfragen, warum das Problem besteht, in welchen Situationen das Kind damit konfrontiert ist, ob andere Kinder ähnliche Erfahrungen gemacht haben. Auf diese Weise regen die Fachkräfte die Kinder zum Erzählen, Schildern, Begründen, Erklären bzw. Berichten an sowie zur Reflexion ihrer Erfahrungen. Außerdem sorgen sie dafür, dass das Problem oder der Wunsch eines Kindes für die Gruppe verständlich und emotional wichtig wird, so dass alle sich einfühlen können. So wird die gesamte Gruppe dazu motiviert, das Anliegen gemeinsam gründlich zu durchdenken und zu diskutieren.

Die zentrale Phase für die Betrachtung von Argumentation ist die Themenbearbeitung. Gemeinsam mit den Fachkräften diskutieren die Kinder nun Ideen zur Erfüllung eines Wunsches bzw. zur Lösung eines Problems. Dabei 
prüfen sie immer auch argumentativ die Tauglichkeit der gefundenen Lösungen. In unserem Beispiel hatte Pauline beklagt, dass die Tücher oft „zerknotet“ sind, und nun fordert die Fachkraft die Gruppe auf, Lösungen zu suchen (Z. 11). Sie benutzt dafür eine Formulierung, die im Korpus sehr häufig vorkommt (Beispiel 02, Teil 2, Zeile 11):

\begin{tabular}{|c|c|c|}
\hline 11 & PFk : & was können wir denn da MACHen?|| \\
\hline 12 & Anton: & [ ich wÜRde (.) daRAN \\
\hline 13 & ?: & [da müssen wir denJEnigen(.)ähm] \\
\hline 14 & Pauline: & wir WISsen aber ja NICHT wer_s WAR; || \\
\hline 15 & & (.) wie sollen wir_s denn WISsen; || \\
\hline 16 & Anton: & dann müssen wir ihn eben !FRA: !gen; || \\
\hline 17 & ?: & {$[(\mathrm{JA}$ aber ...) } \\
\hline 18 & Oskar: & [es SAGT ja nicht] JEder die WAHRheit;।| \\
\hline 19 & viele: & $(\ldots)$ \\
\hline 20 & Pauline: & [es würde KEIner sagen auch wenn er_s WAR;।] \\
\hline 21 & & würde er_s nicht SAgen.|| \\
\hline
\end{tabular}

Beispiel 2: Gesprächskreis 1 (00:01:12): Paulines Stolperstein „Zerknotete Tücher“ (Teil 2)

Bei der Themenbearbeitung (die bei diesem Thema ca. 2 min dauert) entwickeln die Kinder Routinen in lösungsorientierter Argumentation: Sie begründen Anliegen und Lösungsvorschläge, markieren Strittigkeit, wägen Lösungsmöglichkeiten ab und prüfen sie auf Passgenauigkeit für Problem und Adressaten. Sie bringen Beispiele an und nehmen sogar Einwände selbst vorweg, sie übernehmen die Perspektive anderer, verhandeln Kompromisse und bedenken Konsequenzen. Die Fachkräfte moderieren und steuern diesen Prozess zurückhaltend, behutsam und kontextsensitiv. In unserem Beispiel 2 (Teil 2) stellt die Fachkraft nur die Eröffnungsfrage, im weiteren Verlauf reden ausschließlich die Kinder.

Oft wird durch die Diskussion eine möglichst praktikable Lösung gefunden (Entscheidung und Lösungsabsicherung). In unserem Beispiel schlägt die Fachkraft vor, noch einmal mit allen zu sprechen, dass sie die Tücher nicht verknotet liegen lassen (Beispiel 02, Teil 3): 


\begin{tabular}{|c|c|c|}
\hline 61 & $\begin{array}{l}\text { PFk: ( (zu } \\
\text { Pauline)) }\end{array}$ & $\begin{array}{l}\text { aber wenn dich das so ÄRgert können wir ja nochmal } \\
\text { mit ALlen drüber [sprechen und SAgen] }\end{array}$ \\
\hline 62 & viele: & ]$\quad[\quad(\ldots)$ \\
\hline 63 & PFk : & {$[\mathrm{HÖR}$ mal; |] } \\
\hline 64 & & und SAgen nochmal dass das ÄRgerlich ist, | \\
\hline 65 & & wenn man damit sPIElen will, I \\
\hline 66 & & und da hundert KNOten drinne sind, I \\
\hline 67 & & (.) dass DIE kinder die da KNOten REINmachen, \\
\hline 68 & Oskar: & jeNAU; \\
\hline 69 & & wieder RAUSmachen müssen.|| \\
\hline 70 & PFk: & (.) geNAU die dann auch wieder RAUSmachen, I \\
\hline 71 & & $\begin{array}{l}\text { und das dann auch ORdentlich so wie sie_s geNOMmen } \\
\text { haben auch wieder in die ECke legen.। }\end{array}$ \\
\hline 72 & & (.) WÄre das was?|| \\
\hline
\end{tabular}

Beispiel 2: Gesprächskreis 1 (00:01:12): Paulines Stolperstein „Zerknotete Tücher“ (Teil 3)

Üblicherweise sichern die Fachkräfte die gefundenen Lösungen ab; hier z. B. mit dieser Formulierung: WÄre das was?||, und greifen im nächsten Gesprächskreis Thema und Lösung noch einmal auf. Auf diese Weise setzen sie den Leitgedanken der gesellschaftlichen Teilhabe um: Die Gesprächskreise wirken in den Kita-Alltag hinein.

Mit der Analyse der Gesprächskreise gewinnen wir empirisch basierte und alltagsnahe Erkenntnisse darüber, wie fünf bis sechsjährige Kinder eigene Anliegen sprachlich-kommunikativ miteinander verhandeln, welche Entwicklungen sich speziell im Begründungshandeln vollziehen und mit welchen kommunikationsförderlichen Strategien pädagogische Fachkräfte diese Entwicklung begleiten können. Das besondere gesprächs- und speziell argumentationsförderliche Potential dieses Formats liegt darin, dass die Kinder hoch motiviert eigene Anliegen diskutieren und dass ein kooperativer Rahmen geschaffen wird, der die Kinder einlädt zu lustvollem, kreativem Austausch von Argumenten ohne direkte Konfrontation (ausführlicher Bose/Kurtenbach 2019 und Bose/ Hannken-Illjes/Kurtenbach 2020). Viele Kinder entwickeln sich während des Projekts zu kompetenten Akteuren in dem Format. Gesteuert und gefördert wird dies durch die Gesprächsmoderation der pädagogischen Fachkräfte, die an den Ressourcen der Kinder orientiert ist. Die Fachkräfte berichten, dass sich 
die beschriebenen argumentativen Praktiken zum Teil auch auf den Kita-Alltag übertragen. Zum Beispiel bezeichnen die Kinder herausfordernde Situationen oder Probleme im Alltag spontan als „Stolpersteine“. Anhand der Daten können wir also sowohl die Entwicklung der kindlichen Gesprächskompetenz beobachten, z. B. Partizipation und Argumentation, Sprechen über Emotionen, Partnerorientierung, als auch das sprachförderliche Potenzial der Moderation durch die Fachkräfte analysieren und darauf aufbauend empirisch basierte und alltagsnahe Fortbildungen konzipieren.

\section{$4 \quad$ Ausblick}

Gemäß dem Selbstverständnis der Sprechwissenschaft als anwendungsorientiertes akademisches Fach führen wir in unserer Arbeit die Beschreibung, Analyse und Optimierung von Kommunikationsprozessen in der Kita zusammen, mit dem Ziel einer möglichst hohen Interaktionsqualität. Wichtig ist uns auch, die Studierenden im Masterstudiengang Sprechwissenschaft möglichst früh an den Praxiskooperationen zu beteiligen. Das stellt sich als Gewinn für alle Beteiligten heraus und generiert beständig neue Ideen. So haben wir in diesem Jahr das Projekt „Miteinander essen - miteinander sprechen“ begonnen. Wir wollen die Kommunikation der Kinder und pädagogischen Fachkräfte während der Essenssituationen in den Kitas beleuchten. Dazu befragen wir alle Beteiligten, führen videobasierte Untersuchungen durch, um das Sprachbildungs- und Förderpotential dieser Situationen zu erfassen. Außerdem werden wir aus den Erkenntnissen der Analysen des Gesprächskreisprojektes „Stolpersteine \& Wunschsterne“ Fortbildungen für weitere Kitas konzipieren.

Wir danken unseren Projektpartnereinrichtungen, allen pädagogischen Fachkräften, die an den Schulungen teilgenommen haben, sowie unseren Studierenden und vor allem den beteiligten Kindern. 


\section{Literatur}

Abschlussbericht des Landesmodellprojekts „Sprache fördern - Erprobung und Multiplikation von Methoden der Sprachförderung in Kindertagesstätten“ (2011). Berufsbildungswerk Leipzig für Hör- und Sprachgeschädigte gGmbH. http:// www.bbw-leipzig.de/fileadmin/sprache_foerdern/Abschlussbericht/ Abschlussbericht_e-book.pdf

Arnold, David H./Lonigan, Christopher J./Whitehurst, Grover J./Epstein, J. N. (1994): Accelerating language development through picture book reading: Replication an extension to a videotape training format. In: Journal of Educational Psychology, 86, 235-243. Zit. nach Ruberg, Tobias/ Rothweiler, Monika (2012): Spracherwerb und Sprachforderung in der KiTa. Stuttgart: Kohlhammer, 15; S. 157.

Bose, Ines/Hannken-Illjes, Kati/Kurtenbach, Stephanie (2020): und was MACHen wir da jetzt um das problem zu LÖsen? \| Argumentation im Kindergarten und ihre Förderung in einem Gesprächskreisprojekt einer Kindertagestätte. Gesprächsforschung - Online-Zeitschrift zur verbalen Interaktion (ISSN 16171837) Ausgabe 21 (2020), S. 1-29. <www.gespraechsforschung-ozs.de>

Bose, Ines/Kurtenbach, Stephanie/Nixdorf, Sophie (2013): Formen und Funktionen des Sprechausdrucks in Gesprächen zwischen Erzieherinnen und Kindern. In: Kurtenbach, Stephanie/Bose, Ines (Hg.): Gespräche zwischen Erzieherinnen und Kindern. Beobachtung, Analyse, Förderung. Frankfurt a. M. u.a.: Peter Lang, S. 67-100. (HSSP 47).

Bose, Ines/Kurtenbach, Stephanie (2014): Stimmlich-artikulatorischer Ausdruck in der Kita: Erzieherinnen betrachten mit zwei- und vierjährigen Kindern ein Bilderbuch. In: Barth-Weingarten, Dagmar/Szczepek-Reed, Beatrice (Hg.): Prosodie und Phonetik in der Interaktion - Prosody and phonetics in interaction. Verlag für Gesprächsforschung: Mannheim, 136-161. <http://verlag-gespraechsforschung.de/2014/barth.html>

Bose, Ines/Kurtenbach, Stephanie (2019): „Stolpersteine und Wunschsterne“ Förderung von Partizipation und Gesprächsfähigkeit in der Kita. In: Bose, Ines/Hannken-Illjes, Kati/Kurtenbach, Stephanie (Hg.): Kinder im Gespräch. Berlin u.a.: Frank\&Timme, S. 113-148. (SSP 16). (u. Mitarb. v. Kerstin Kreße, Friederike Küstner, Constanze Müller).

Buschmann, Anke (2009): Heidelberger Elterntraining zur frühen Sprachforderung. Trainer- manual. München: Elsevier.

Kannengieser, Simone (2019): Sprachentwicklungsstörungen. Grundlagen, Diagnostik und Therapie. 4., aktualisierte Auflage. München: Urban \& Fischer.

Kupietz, Michaela (2013): Untersuchungen von Schulungseffekten auf das Gesprächsverhalten von Erzieherinnen. In: Kurtenbach, Stephanie/Bose, Ines (Hg.): Gespräche zwischen Erzieherinnen und Kindern. Beobachtung, Analyse, Förderung. Frankfurt a. M. u.a.: Peter Lang, S. 51-66. (HSSP 47). 
Kurtenbach, Stephanie (2009): Spielst du mit mir sprechen? Trainingsprogramm zur Förde- rung der kindlichen Sprachentwicklung. Arbeitsmaterial für Erzieherinnen in der Qualifizierungsreihe des Landesmodellprojekts „Sprache fördern“. Freistaat Sachsen. Qualifizierung- 5. Fortbildungstag: Sprachförderung/Methoden Teil 2. (Mskr.).

Kurtenbach, Stephanie (2011): Einblick in die Qualifizierungsmaßnahme des Landesmodellprojekts Sprache fördern, Sachsen: Methoden der Sprachförderung. In: Sievert, Ulrike/Voigt-Zimmermann, Susanne (Hg.): Klinische Sprechwissenschaft. Aktuelle Beiträge aus Wissenschaft, Forschung und Praxis. (HSSP 35). Frankfurt a. M. u.a.: Peter Lang, S. 127-133.

Kurtenbach, Stephanie/Kreutzer, Franziska/Eilers, Annika/Gräfe, Simone/Noe, Elisa/Zachow, Paulin (2011): Beo@ Beobachtungsbogen für frühe kommunikative Fähigkeiten. <https://kita-halle.de/projekte/kinderleicht-sprechen/artikel/ beo-beobachtungsbogen-fuer-fruehe-kommunikative-faehigkeiten>

Kurtenbach, Stephanie/Bose, Ines/Koch, Elena/Kreft, Hannah (2013): Gesprächskreise in Kindertagesstätten - Anlass zur Kommunikationsförderung? In: Kurtenbach, Stephanie/Bose, Ines (Hg.): Gespräche zwischen Erzieherinnen und Kindern. Beobachtung, Analyse, Förderung. Frankfurt a. M. u.a.: Peter Lang, S. 111-118. (HSSP 47).

Kurtenbach, Stephanie/Bose, Ines/Thieme, Tabitha (2013): Gemeinsam ein Bilderbuch anschauen. Untersuchung zum Gesprächsverhalten von Erzieherinnen. In: Kurtenbach, Stephanie/Bose, Ines (Hg.): Gespräche zwischen Erzieherinnen und Kindern. Beobachtung, Analyse, Förderung. Frankfurt a. M. u.a.: Peter Lang, S. 23-50. (HSSP 47).

Kurtenbach, Stephanie/Bose, Ines (2014): Sprachförderstrategien im Kita-Alltag - Analysen von Gesprächen zwischen Fachkräften und Kindern. In: Sallat, Stefan/Spreer, Marcus/Glück, Christian (Hg.): Sprache professionell fördern: kompetent-vernetzt-innovativ. Tagungsband des 31. Bundeskongresses der Deutschen Gesellschaft für Sprachheilpädagogik (dgs). Idstein: Schulz-Kirchner, S. 303-310. (Sprachheilpädagogik aktuell).

Kurtenbach, Stephanie/Kreutzer, Franziska/Eilers, Annika/Gräfe, Simone/Noe, Elisa/Zachow, Paulin (2019): Kommunikative Fähigkeiten im frühpädagogischen Kontext beobachten - Entstehung und Evaluierung eines Erhebungsinstruments (Beo). In: Bose, Ines/Hannken-Illjes, Kati/Kurtenbach, Stephanie (Hg.): Kinder im Gespräch. Berlin u.a., : Frank\&Timme, S. 271-287. (SSP 16).

Manolson, Ayala (1992): It takes two to talk. A parent's guide to helping children communicate. Imagine Print: Toronto.

Möller, Delia (2006): Schritte in den Dialog. Ein Eltern- Kind- Programm für Familien mit sprachentwicklungsverzögerten Kindern. In: Forum Logopädie, 1 (20), S. 6-11.

Ruberg, Tobias/Rothweiler, Monika (2012): Spracherwerb und Sprachforderung in der KiTa. Kohlhammer: Stuttgart. 
Schäuble, Lisa (2020): Mit mehrsprachigen Kindern im Gespräch sein. <https:// kita-halle.de/projekte/kinderleicht-sprechen/artikel/forschungskooperationen> Selting, Margret et al. (2009): Gesprächsanalytisches Transkriptionssystem 2 (GAT 2). In: Gesprächsforschung - Online-Zeitschrift zur verbalen Interaktion (ISSN 1617-1837). 10, S. 353-402. <www.gespraechsforschung-ozs.de> (15.10.2020). Siegmüller, Julia/Fröhling, Astrid (2010): Das PräSES-Konzept. Potenzial der Sprachförderung im Kita-Alltag. Elsevier: München.

\section{Legende der Notationszeichen} (orientiert an GAT2; vgl. Selting et al. 2009)

PFk pädagogische Fachkraft

| bzw. || prosodisch nicht abgeschlossene bzw. abgeschlossene Phrase

(.) Mikropause, geschätzt, bis ca. 0.2 Sek. Dauer

? bzw. , hoch bzw. mittel steigende Melodiebewegung am Phrasenende

. bzw. ; tief bzw. mittel fallende Melodiebewegung am Phrasenende

(...) unverständliche Passage, ggf. mit vermutetem Wortlaut

[...] Überlappungen und Simultansprechen 

Baldur Neuber, Halle (SaAle)

\title{
Miteinander reden im digitalen Kontext
}

Zusammenfassung: Seit fast zwanzig Jahren wird in Halle die Kommunikation in digitalen Kontexten erforscht. Gegenwärtig gibt es enorme Änderungen in der Telekommunikationsbranche. Zunehmend wird die multimediale Kommunikation im großen Maßstab angeboten. Leider liegt der Focus von Forschung und Entwicklung fast ausschließlich auf technischen und wirtschaftlichen Sachverhalten. Die menschliche Kommunikation spielt eine marginale Rolle. Dieses Desiderat wird durch die Sprechwissenschaft gefüllt und in diesem Aufsatz erörtert.

Stichworte: Digitale Kommunikation, Multimedia, Soziale Medien, Omnichannel, Mensch-Maschine-Mensch-Kommunikation

\begin{abstract}
For almost twenty years, Halle has been researching communication in digital contexts. There are currently huge changes in the telecommunications industry. Multimedia communication is increasingly being offered on a large scale. Unfortunately, the focus of research and development is almost exclusively on technical and economic issues. Human communication plays a marginal role. This desideratum is filled by the speech science and discussed in this essay.
\end{abstract}

Keywords: Digital communication, Multimedia, Social Media, Omnichannel, Human-machine-human-communication

\section{$1 \quad$ Was war?}

Die sprechwissenschaftliche Erforschung der zwischenmenschlichen Kommunikation in digitalen Kontexten besitzt in Halle inzwischen eine fast zwanzig- 
jährige Tradition. Die Zahl der abgeschlossenen Qualifikationsarbeiten und Publikationen liegt im hohen zweistelligen Bereich. Einen sehr guten Gesamtüberblick über die zentralen Projekte findet man bei Pietschmann (2017). In jüngster Zeit verändert sich die digital vollzogene Sprechkommunikation erheblich. Zeit also, eine kurze Bilanz zu ziehen und eine Projektion in die Zukunft zu wagen.

Unsere ursprüngliche Motivation zur Erforschung der digitalisierten Kommunikation ergab sich aus direkten Anfragen der Telekommunikationsindustrie. Sie betreibt allein in Deutschland tausende von Telekommunikationszentren, die früher als Callcenter bezeichnet wurden und sich gegenwärtig überwiegend zu „Omni-Channel-Centern“ qualifiziert haben. Der praktische Unterschied besteht darin, dass Callcenter zumeist nur professionelle Telefonie absichern, während „Omni-Channel-Center“ die gesamte Außenkommunikation der Auftraggeber sichern, insbesondere die Schrift- und Bildkommunikation in Internet und in Social Media.

$\mathrm{Zu}$ Beginn unserer Forschung hatte die Branche ein schlechtes Image in der Öffentlichkeit, das v.a. durch unseriöses Telefonmarketing und „Kaltakquisen“, also durch kundenseitig unerwünschte Verkaufsanrufe, verursacht wurde. Bereits damals waren aber die meisten Telefonate Inboundgespräche, also Anrufe der Kunden bei Unternehmen bzw. Callcentern. Das Schmuddelimage war und ist statistisch betrachtet unberechtigt, denn Telekommunikationscenter (TKC) leisten überwiegend wertvolle Arbeit, von der technischen Hotline bis hin zur 112-Notrufannahme. Kein Grund also für die Sprechwissenschaft, sich abzuwenden. Der Chef eines großen TKC sagte mir einmal: „99\% der Arbeit verlaufen gut und reibungslos und das $1 \%$, bei dem etwas schief geht, steht dann in der Zeitung." Dem kann man nur zustimmen.

Durch Gesetzesänderungen (Datenschutz-Grundverordnung, DSGVO, Internetquelle 1) ist der Belästigungsmarkt unerwünschter Kaltakquisen ohnehin weitgehend zusammengebrochen. Im Falle von Verstößen und juristisch grenzwertigen Methoden hat die Kundschaft sehr gute Chancen, diese rasch zu stoppen. Das negative Image ist dadurch leider noch längst nicht ausgeräumt. Der Begriff des Omni-Channel-Centers ist in der Bevölkerung nahezu unbekannt und die Bezeichnung „Callcenter“ wird von vielen Menschen mit Spamschleudern unerwünschter Telefonanrufe assoziiert. 
Desungeachtet wächst der Markt an essenziell wichtiger professioneller Telefonie mit hohem Nutzen, auch wenn dies in den Medien nahezu nicht thematisiert wird.

Fast alle Großunternehmen und Institutionen überantworten ihre Außenkommunikation den Telekommunikationszentren bzw. Omni-ChannelCentern. Die Universitäten hinken - wie so oft - hinterher. Hier können Studierende versehentlich direkt an Professorinnen und Professoren sowie wissenschaftliche Mitarbeiter*innen gelangen, obwohl sie eigentlich nur ein Verwaltungsanliegen haben. Wenn man etwas über hervorragende Kommunikationstechnologie (einschl. der menschlichen Kommunikation) lernen will, muss man sich z. B. Siemens oder T-Systems anschauen. Wenn man lernen will, wie es vor 20 Jahren ging, sind die meisten staatlichen Hochschulen und Universitäten "gute“ Negativbeispiele. Insgesamt sind die digitalen Kommunikationssysteme des öffentlichen Dienstes in großen Teilen desaströs. So ist z. B. der „Beitragsservice“ von ARD, ZDF und Deutschlandradio nicht einmal per E-Mail erreichbar. Stattdessen steht im Briefkopf. „Sie erreichen uns unter Telefon ... und Telefax..." (persönliche Korrespondenz am 19.08.2020). Telefax ist vermutlich für junge Menschen entweder eine Lachnummer oder eine unbekannte Größe. Jedenfalls kann ich mich nicht erinnern, in den vergangenen zwanzig Jahren mit einem Studierenden oder Kollegen per „Telefax“ kommuniziert zu haben. Hier lohnt es sich für Institutionen aller Art, künftig etwas Geld in die Hand zu nehmen, und Teile der Kommunikation den Profis zu überlassen. Inzwischen werden bei Bedarf zahlreiche Kommunikationsformen und -inhalte übernommen, für Exzentriker sicher sogar „Telefaxe“.

Alle unsere bisherigen Forschungsergebnisse wurden kontinuierlich publiziert (z. B. Neuber 2016). Schwerpunkt bildeten die Sprechwirkungsforschung (z. B. Neuber/Hirschfeld 2013) und die experimentalphonetische Forschung (z. B. Beier/Pietschmann 2016). In jüngerer Zeit kam es immer öfter zu interdisziplinären Vorhaben, die u.a. automatisierte Analysen (Walther et al. 2015 und 2016) zum Thema hatten und somit unser Wissen in eine Richtung erweitern, die die Sprechwissenschaft nur mit Hilfe der Informatiker*innen und Informationstechniker*innen beschreiten kann.

Seit Kurzem vollziehen sich enorme Änderungen in der Telekommunikationsbranche. Zunehmend werden multimediale Kommunikationsangebote, 
insbesondere Social-Media-Plattformen, für die Großkunden aufbereitet. Alle erdenklichen Zielgruppen sollen gleichsam erreicht werden. Die Endkunden werden auf den Wegen abgeholt, auf denen sie sich gerade befinden. Aus Callcentern werden somit die bereits genannten Omni-Channel-Center. Aus meiner Sicht bringt diese Tendenz neben Bequemlichkeitsvorteilen auch eine enorme kommunikative Redundanz mit sich, die gefährliche Entwicklungen induziert. Darüber wird im nächsten Kapitel noch zu berichten sein.

Die beschriebene Entwicklung eröffnet zugleich die Chance zu einer inhaltlichen Neuausrichtung der Telekommunikationsbranche und zum Aufbau eines besseren Images.

\section{Was ist?}

Das hallesche Forschungsteam hat über 16 Jahre lang alle denkbaren Informationen über die Telekommunikations- und Callcenterbranche gesammelt und alle wichtigen Messen und Kongresse besucht, allen voran die Call Center World (Internetquelle 2).

Diese ist eine der weltweit größten Messen der Telekommunikationsbranche und zugleich eine gigantische Kongressmesse. Dabei fällt bis in die Gegenwart hinein schmerzlich auf, dass der Focus von Forschung und Entwicklung fast ausschließlich auf technischen und wirtschaftlichen Sachverhalten liegt, die menschliche bzw. zwischenmenschliche Kommunikation spielt eine sehr marginale Rolle. Man kann den Eindruck gewinnen, dass die Industriestrategen glauben, sie könnten das miteinander Reden verbessern, indem sie noch eine App und noch eine App und noch eine ... entwickeln. Hier liegt meiner Ansicht nach ein gefährlicher Irrtum vor. Wenn ich massenhaft Messengerdienste bzw. -apps wie z. B. „Facebook“, „Instagram“, „Skype“, „Telegram“, „WhatsApp“ usw. auf mein Endgerät lade und all diese nutze, dann ist das etwa so, als ob ich zehn Postausgangs- und Briefkästen an meine Hauswand nageln ließe. Statt einen Postkastens, wie bisher, muss ich zehn öffnen und einsehen und womöglich wird dann auch noch erwartet, dass ich mich schnellstens rückäußere. Wenn ich Pech habe, befinden sich in der Hälfte der „Kästen“ die gleichen Nachrichten, so dass zunächst eine umfassende „E-Müllabson- 
derung" fällig wird. Bei meinen Kolleginnen und Kollegen beobachte ich in jüngster Zeit, dass zwischen den Kanälen wild hin und hergeschaltet wird, also einmal eine Nachricht per SMS, dann per E-Mail, dann per WhatsApp usw. Da möchte man mit Herbert Grönemeyer fragen: „Was soll das?“. Die Herstellung neuer Kommunikationskanäle und Apps scheint sich zu verselbstständigen. Das daraus resultierende Problem könnte man wie folgt zusammenfassen: Die technischen Möglichkeiten entwickeln sich derzeit viel schneller, als die Menschheit sie sinnvoll zu nutzen vermag. Dabei können all diese wunderbaren Apps und Geräte nur das eine: Informationen übertragen und evtl. generieren. Die inflationäre Nutzung von Informationstechnologien führt zu einem gefährlichen Anwachsen der Entropie. Wir bewegen uns also von der Struktur in Richtung Chaos. Interessante Überlegungen finden wir u.a. bei Feustel (2018), auf dessen Lektüre ich hier ausdrücklich verweisen möchte.

Was aber ist die Antwort der Industrie auf diese Entwicklung? Das wissen Sie alle: (Noch) mehr desselben - 5G muss her. Natürlich ist es nicht weiter schlimm, wenn unser Informationsmüll noch schneller um die Welt transportiert werden kann, aber das löst nicht das Problem. Dieses ist v.a. qualitativer Natur. Wir brauchen nicht in erster Linie mehr Apps und mehr Megabit/s, sondern wir brauchen Sinn, Verstand und menschliche Emotionen in den Inhalten und ein „Weg vom Kommunikationschaos - zurück zur Struktur!“ Hier gibt es ein riesiges unbeackertes Gebiet für die Sprechwissenschaft.

Die gegenwärtige elektronische Medienqualität (und damit Kommunikationsqualität) ist aus meiner Sicht äußerst defizitär und dem menschlichen Miteinander oftmals eher zuwider als förderlich. Einige Probleme werden im Folgenden stichwortartig aufgelistet:

- reduktionistisches nonverbales Feedback sowie 2-D-Technologie und kleine ruckelige Bilder in Videokonferenzsystemen etc.,

- Konzentrationsüberforderung durch schlechte digitale Tonübertragungen,

- Bewegungsmangel durch fehlende Proxemik,

- „Däumelinchen“-Texteingabe in Smartphones u. Tablets (Zwei-Daumen-Suchsystem) statt 10-Finger-System, 
- Aufmerksamkeitsbindung an die virtuelle Welt und Aufmerksamkeitsreduktion gegenüber der physischen Welt,

- „Partnerillusion“ durch Bots u.ä.,

- Anreiz zur ständigen Erreichbarkeit, die den natürlichen Biorhythmen widerspricht,

- Informationsüberflutung und minderwertige Information (z.B. "Spam"),

- fehlende taktile und olfaktorische Information,

- fehlendes Abstandsgefühl.

Sicher ließe sich diese Liste noch erheblich fortführen, aber die wesentlichen Punkte sind hier vermutlich genannt. Feustel geht in seiner Kritik noch weiter, indem er die „neue Illusion vom Menschen als informationsverarbeitendes System, das bald nachgebaut werden kann..." $(2018,141)$ diagnostiziert. Dabei ist die Frage, ob künftig Maschinen wie Menschen kommunizieren können für mich eindeutig nachrangig gegenüber der Frage, wer mit wem zu welchem Zweck kommunizieren will. Zudem ist zu fragen, wie die von mir geschilderte softwareverursachte Redundanz in Effizienz verwandelt werden kann. Nach meiner Auffassung besteht kommunikative Qualität vor allem darin, dass Menschen ihre Denkinhalte, Auffassungen, Wertungen, Emotionen usw. einander mitteilen und möglichst zur gemeinsamen Sache werden lassen (vgl. Geißner 1988, 45). Natürlich können die Menschen Computer, Tablets und Smartphones en gros verwenden und mit zahllosen Apps belegen. Aber können sie damit effektiver oder gar „besser“ kommunizieren? Das bezweifle ich - angesichts der geschilderten Defizite der digitalisierten Kommunikation - sehr.

Aus sprechwissenschaftlicher Sicht ist besonders problematisch, dass die Erforschung von Kommunikation sich fast ausschließlich auf „Technisches" konzentriert, und dass die Maschine-Mensch-Kommunikation bzw. Mensch-Maschine-Mensch-Kommunikation zurückbleibt. Es ist eben nicht damit getan, dass Siri, Alexa und Co. nahezu jedes gesprochene Wort dahingehend „verstehen“, dass sie es im Internet suchen und dann das Suchergebnis herunterplappern. Das ist gewiss nicht die Art von Kommunikation, die wir zum Leben wirklich brauchen. Menschlicher Informationsaustausch ist eben weitaus mehr als das Übertragen von Signalen und/oder Codes vom Sender 
zum Empfänger über einen möglichst störungsfreien Kanal. Vielmehr konstruieren und generieren unsere Gehirne das, was wir als Information aufnehmen, verarbeiten und absenden. Diese Art Information ist gewollt subjektiv bzw. intersubjektiv. Hierzu ein Beispiel: Wenn ich mit den Sinnesleistungen und Fähigkeiten der heutigen künstlichen Intelligenz durch einen Wald gehen würde, um mir Pilze anzuschauen, dann würde mir vielleicht der lateinische und deutsche Name eines jeden Pilzes sofort einfallen. Wenn ich den gleichen Spaziergang jedoch als Mensch unternehme, dann erlebe ich Farben, Formen, Düfte, Gefühle, gedankliche Assoziationen und vieles mehr. Die rationale Benennung und Beschreibung ist ein winziger Teil des Informationsgeschehens. Das gilt zugleich für die (Sprech)kommunikation. Wenn ich über einen Pfifferling rede, assoziiere ich ein Ensemble von visuellen, haptischen, olfaktorischen und sogar akustischen Eindrücken: Ein schlecht geputzter Pfifferling knirscht beim Essen. Dass dieser Pilz auch Cantharellus cibarius genannt wird, spielt allenfalls eine kleine Teilrolle. Offen gestanden wusste ich das bis heute nicht und habe es nur für diesen Aufsatz nachgeschlagen, obwohl ich seit Jahrzehnten gern Pfifferlinge esse.

Kurz zusammengefasst: Die gegenwärtig überall kursierende Idee, das menschliche Gehirn sei eine Art biologischer Computer mit „vernetzten“ Elementen, halte ich für äußerst reduktionistisch. Das ist - wenn überhaupt - ein Gleichnis für die anatomischen und physiologischen Verhältnisse in den Gehirnen, nicht aber für die Funktion. Hier können wir noch einmal Feustel $(2018,186)$ bemühen: „Die substantielle Differenz zwischen menschlichem Denken und Fühlen auf der einen Seite und (informationstechnischem, Erg. BN) Prozessieren auf der anderen wird eindrücklich sichtbar. Die Leistungsfähigkeit der Maschine spielt dafür keine Rolle.“

\section{$3 \quad$ Was könnte sein?}

Sprechkommunikation in digitalen Kontexten ist für uns Sprechwissenschaftlerinnen und Sprechwissenschaftler von höchstem Interesse, da sie die direkte leibhaft vollzogene Kommunikation zunehmend ergänzt und ablöst. Die 
jüngste Corona-Pandemie hat diesen Prozess nochmals erheblich beschleunigt. Unsere große Forschungsaufgabe besteht darin, die Differenzen zwischen digital geführter Kommunikation und menschlicher Kommunikation viel präziser verstehen zu lernen, als dies bisher der Fall ist. Was genau müssen z. B. Sprachassistenzen können, damit ihre „Kommunikation“ brauchbar wird? (Auf dem heutigen Stand sind sie für mich hauptsächlich Spielerei. Ich weiß auch nicht, warum ich mich mit meinem Auto „unterhalten“ soll, statt einfach nur einige der wenigen fahrtrelevanten Bedienelemente zu betätigen. Was ist an diesen unsinnigen Pseudodialogen der echte Mehrwert?) In welchen Fällen können wir uns auf die Kommunikation mit der und durch die Maschine verlassen? In welchen Fällen ist sie eher ein schlechtes Surrogat und wo ist sie überhaupt nicht sinnvoll einsetzbar? Diesen und ähnlichen Fragen sollten wir uns in den nächsten Jahren stellen.

Darüber hinaus muss die Sprechwissenschaft aufklärend und andragogisch tätig werden. Hier kann man bereits ohne groß angelegte Studien vieles über Plausibilitäten abklären:

- Wie wird zwischenmenschliche Kommunikation in digitalen Kontexten effizient?

- Wann lenkt uns das Digitale vom „echten analogen“ Leben eher ab?

- Welche technischen Voraussetzungen sind für gutes miteinander Reden in digitalen Umfeldern erforderlich?

- Wie wirkt sich digital untersetzte Kommunikation in fachkommunikativen bzw. sektoralen Umfeldern aus?

Vermutlich wird es irgendwann gelingen, menschliches Sprechdenken maschinell zu simulieren. Aber eben auch das ist eine Simulation. Wenn wir einem Affen eine Weste anziehen, wird dieser dann dadurch wirklich menschlicher? Unser Ziel kann nicht - wie bisher - darin bestehen, auf immer neue Hardund Software und deren Mehrwert zu hoffen. Es muss uns hingegen gelingen, wieder die (kommunikative) Herrschaft über die Technik zu übernehmen und den Schalter im richtigen Moment auch für längere Zeit auf „Off“ setzen zu können. 
Lassen Sie mich mit einem Gleichnis schließen: Der Schriftsteller E. M. Forster veröffentlichte 1908 die dystopische Erzählung „Die Maschine steht still“, die erst 2016 ins Deutsche übertragen und kürzlich in einem grandiosen Hörspiel (Kubin 2019) aufbereitet wurde. In Forsters Fiktion ist die Erdoberfläche durch Umweltzerstörung und Krankheitserreger unbewohnbar geworden. Deshalb leben die Menschen in winzigen unterirdischen sechseckigen Waben, allerdings mit allem Komfort. Sie kommunizieren fast ausschließlich auf elektronischem Wege. Der gesamte Lebensalltag wird durch die „Maschine“ perfekt geregelt. Das Handbuch der Maschine ist die Bibel dieser Menschheit. Persönliche Begegnungen und Gespräche sind zwar nicht ausdrücklich verboten, werden aber als unmaschinell verpönt. Zudem sind die Menschen nicht mehr an die intensiven Eindrücke direkter Begegnungen gewöhnt und haben deshalb sogar Angst davor. Stattdessen generieren sie "Ideen“, die als Menschheitserkenntnisse in den Speichern der Maschine abgelegt werden. Es scheint mir, als habe Forster die „digitalen Zeiten“ und die "Coronazeiten“ nahezu perfekt vorausgeahnt. Die kluge Maschine im Roman gewinnt irgendwann die Erkenntnis, dass das beschriebene Geschehen weder in eine erstrebenswerte menschliche noch in eine technizistisch-maschinelle evolutionäre Perspektive führt. Der Ausgang bleibt offen: Die Maschine schaltet sich selbst ab und hinterlässt die Menschheit in einem „narkoleptischen“ Schlummerzustand.

Auf die Weisheit der Maschine würde ich mich nicht verlassen, ebenso wenig auf die unserer Gegenwartspolitiker*innen. Allerdings glaube ich an die Kolleginnen, Kollegen und Studierenden unseres Faches. Wer sollte die soziale (und damit zugleich sprechkommunikative) Degeneration unserer Gesellschaft besser verhindern können als wir?

\section{Literatur}

Beier, Frances Theres/Pietschmann, Judith (2016): CRM-basierte Sprechwirkungsforschung im Kontext der professionellen Telefonie - eine Pilotstudie. In: Baldur Neuber/Judith Pietschmann (Hrsg.): Dialogoptimierung in der Telekommunikation. SSP 9, Berlin: Frank \& Timme, S. 137-153.

Feustel, Robert (2018): Am Anfang war die Information. Verbrecher Verlag. 
Forster, Edward Morgan (1909/2019): Die Maschine steht still. Hörspiel von Felix Kubin u.v.a. CD mit Booklet. NDR Kultur.

Geißner, Hellmut (1988): Sprechwissenschaft. Theorie der mündlichen Kommunikation. Frankfurt a.M.: Scriptor.

Neuber, Baldur (2016): Gesprächsoptimierung in der Telekommunikation unter interkulturellem Blickwinkel. In: Hirschfeld, Ursula/Lange, Friderike (Hrsg.): Phonetische und rhetorische Aspekte der interkulturellen Kommunikation. SSP 7, Berlin: Frank \& Timme.

Neuber, Baldur/Hirschfeld, Ursula (2013): Sprechwirkungsforschung in der professionellen Telefonie. In: Veličkova, Ludmila/Petročenko, Elena (Hg.): Klangsprache im Fremdsprachenunterricht (VII). Forschung und Praxis. Woronesch: Staatliche Universität, S. 66-85.

Pietschmann, Judith (2017): Optimierung von Gesprächen in der professionellen Telefonie. Rhetorische und phonetische Einflussfaktoren auf die Qualität von Telefonverkaufsgesprächen. SSP 12, Berlin: Frank \& Timme.

Walther, Mathias/Neuber, Baldur/Jokisch, Oliver/Mellouli, Ta ïeb (2015): „Towards a Conversational Expert System for Rhetorical and Vocal Quality Assessment in Call Center Talks“. In: SLaTE 2015 - Sixth Workshop on Speech and Language Technology in Education, Leipzig, September 4-5, S. 29-34 ("IS “ISCA Workshop on Speech and Language Technology in Education").

Walther, Mathias/Beier, Frances Theres/Mellouli, Ta ïeb/Neuber, Baldur (2016): Automatische Klassifikation des Ersteindrucks von Sprechereigenschaften bei ungeschulten Hörern. In: Jokisch, Oliver (Hrsg.): Elektronische Sprachsignalverarbeitung (ESSV). Tagungsband der 27. Konferenz. Leipzig: TUDpress.

\section{Internetquellen}

Internetquelle 1: https://eur-lex.europa.eu/legal-content/DE/TXT/?uri=CELEX: 02016R0679-20160504 (Letzter Zugriff: 31.08.2020)

Internetquelle 2: https://www.ccw.eu/kongressmesse/ (Letzter Zugriff: 31.08.2020) 


\section{Visionen und Grenzen der digitalen Kommunikationswelt 4.0 aus sprechwissenschaftlicher Perspektive}

Zusammenfassung: Unsere Kommunikation wird zunehmend durch die Digitalisierung beeinflusst und es bilden sich neue Formen der Interaktion heraus. Daraus ergeben sich Fragen nach dem Umgang mit der rasanten Entwicklung immer neuer Kommunikationstechnologien und nach der Didaktisierung kommunikativer Kompetenzen. Die Sprechwissenschaft beschäftigt sich in verschiedenen Forschungsfeldern mit der digitalisierten Kommunikation, von denen einige im Beitrag vorgestellt und reflektiert werden.

Stichworte: Digitalisierung, KI-Kommunikationstechnologie, Omnichannel-Kommunikation, E-Learning, Mixed Reality

Abstract: Human communication is more and more affected by digitalization, so that new forms of interaction evolve. This gives rise to questions about how to deal with this rapid development of always new communication technologies and about the education of communicative skills. Speech science deals in various fields of research with digitized communication. Some of these fields should be presented and reflected in the article.

Keywords: digitalization, KI communication technology, omnichannel communication, e-learning, mixed reality

Digitalisierung und social media durchziehen inzwischen alle gesellschaftlich relevanten Bereiche - von medizinischer Versorgung über den öffentlichen und privatwirtschaftlichen Dienstleistungssektor bis hin zur Schulbildung. Auch das Miteinanandersprechen in öffentlichen Kommunikationskontexten vollzieht sich zunehmend digital. Was noch vor wenigen Jahren science fiction war, ist heute Kommunikations- und Interaktionsrealität. 
Jüngste Entwicklungen, die in Zusammenhang mit der Covid-19-Pandemie stehen, zeigen, dass das öffentliche Leben nur mittels digitaler Konzepte überhaupt weiter funktionieren konnte. Die Akzeptanz für digitale Alternativen und Versorgungskonzepte steigt weiter. Für den Bereich der Sprachtherapie/ Logopädie beispielsweise belegen Bilda et al. 2020 in einer Studie die wachsende Bereitschaft zum Einsatz digitaler Technologien und Produkte in der therapeutischen Versorgung. Demnach gilt für einen Großteil der Befragten die Teletherapie als das Versorgungsmodell der Zukunft (vgl. ebd. 176). Auch Patient*innen schließen sich diesem Trend zunehmend an.

Wie gehen wir mit der rasanten kommunikations-technologischen Entwicklung um? Welche Potentiale liegen in der Digitalisierung, um mündliche Kommunikation teilhabeförderlich zu optimieren? Welche Visionen haben wir, auch in Bezug auf die Didaktisierung kommunikativer Kompetenzen? Und welchen Beitrag kann und sollte das Fach Sprechwissenschaft leisten, damit das Miteinandersprechen im heutigen, digitalen Zeitalter verantwortlich, kompetent und reflektiert das Miteinanderleben von morgen prägt.

Die Sprechwissenschaft beschäftigt sich in unterschiedlichen Forschungsund Entwicklungsfeldern (z. B. Sprachtherapie, Kundinnendienstleistung, Medien) mit der Digitalisierung von Kommunikation und den damit verbundenen Potentialen, Visionen und Grenzen. Daher war es naheliegend, im Rahmen eines Podiumsgesprächs zum Thema „Visionen und Grenzen der neuen digitalen Kommunikationswelt 4.0“, welches anlässlich der „Kleine Fächer-Wochen“ im Januar 2020 stattfand, mit verschiedenen Akteuren ins Gespräch zu kommen. Gleichzeitig stellte die öffentliche Podiumsdiskussion für das Fach eine reichweitenstarke Plattform dar, um den Anteil sprechwissenschaftlicher Forschung in diesen gesellschaftlich relevanten Anwendungsfeldern aufzuzeigen und die vielseitige Vernetzung unserer Disziplin zu verdeutlichen.

Im Folgenden sollen einige dieser Forschungsfelder vorgestellt, Digitalisierungstendenzen kritisch reflektiert und Beiträge sprechwissenschaftlicher Forschung dargestellt werden: 


\section{Callcenter-/Omnichannel-Kund ${ }^{*}$ innendienstleistung}

\subsection{Einsatz von $\mathrm{KI}$ in der Kund ${ }^{*}$ innendienstleistung}

Der Einsatz künstlicher Intelligenz (KI) ist seit langem status quo in der telefonischen Kundendienstleistung. Seit vielen Jahren sind Kund ${ }^{*}$ innen vertraut mit navigierenden Sprachmenüs, die dem eigentlichen Mitarbeiter*innenkontakt vorgeschalten sind, um Anliegen an die passenden Kanäle / Mitarbeiter*innen weiterzuleiten. Zunehmend etabliert haben sich inzwischen auch KI-basierte, sprachgesteuerte Self-Services, in denen ,virtuelle Agent*innen reale Kund ${ }^{*}$ innenanliegen bearbeiteten. Solche Self-Service-Lösungen dienen einer schnellen und effizienten Bearbeitung einfach zu lösender (sog. low-level) Kund ${ }^{*}$ innenanfragen und stoßen auf eine zunehmende Akzeptanz (vgl. Internetquelle 1), für die wohl v. a. folgende Gründe verantwortlich zeichnen:

- gut funktionierende Spracherkennung (KI), die für Standardanliegen umfangreich trainiert wurde,

- Zeitersparnis durch den Wegfall von Warteschleifen,

- Kombination unterschiedlicher Kommunikationskanäle (Omnichannel), z. B. durch Wechsel von Anruf auf SMS bei Störung (eingehender Anruf),

- realitätsnahes akustisches Setting (Hintergrund- und Tastaturgeräusche), das z. T. auch gezielt auf die Markenidentität von Unternehmen zugeschnitten ist,

- interaktives Setting und an die Gesprächsrealität angepasstes Antwortverhalten der, virtuellen Agenten ' (z. B. durch Disfluenzen, d. h., Einsatz von Häsitationspartikeln um die noch laufende Sprachverarbeitung anzuzeigen) (vgl. Internetquelle 2).

Zu den hier genannten, überwiegend technischen Weiterentwicklungen KI-basierter Self-Services, kommt nach Meinung von Dirk Egelseer, Präsident des CallCenterVerbands Deutschland (vgl. Internetquelle 3), auch ein gewisser ,Sozialisations-Effekt. Viele Kund*innenbiographien schreiben von schlechten Erfahrungen bei der Bearbeitung recht einfacher Anliegen durch die Kund ${ }^{*}$ in- 
nenberater*innen: lange Warteschleifen, mitunter wenig kompetente und befugte Mitarbeiter*innen, Weiterverbindungs-Marathons und mitunter kaum zufriedenstellende Resultate. Das ist die Erfahrung vieler Kund*innen und die damit verbundene Negativ-Erwartung lässt sie bereitwillig (gut und mitunter besser funktionierende) Self-Service-Leistungen nutzen.

Der Trend geht, so der Kenner der Branche Dirk Egelseer, einerseits in Richtung Ausdifferenzierung von KI-Technologien (vgl. Internetquelle 4) andererseits wird die Bearbeitung spezifischer und individueller Kund ${ }^{*}$ innenanliegen nach wie vor in ,Menschenhand' verbleiben. Kundinnen wünschten sich für individuelle Anliegen entsprechend individuelle und hochqualifizierte Beratung realer Kundinnendienstmitarbeiter*innen. Dies setzt auch ein Umdenken in der Branche voraus, die all zu oft auf technische Kommunikationslösungen setzt und derweil die Wertigkeit qualifizierter, individueller und authentischer zwischenmenschlicher Dienstleitungskommunikation aus dem Blick geraten lässt. Hier hat die hallesche Sprechwissenschaft durch die Definition forschungsbasierter Qualitätsstandards für eine ,authentische und empathische Kundenkommunikation' (vgl. Pietschmann 2017a) sowie durch die Entwicklung entsprechender Schulungskonzepte in den vergangenen Jahren einen wichtigen Beitrag geleistet.

Gegenstände künftiger Forschung liegen für uns beispielweise in einer interaktionstheoretisch begründeten Ausdifferenzierung des interaktiven Settings von KI-basierten Self-Services und den darin agierenden, virtuellen Agent ${ }^{\star}$ innen. Auch das Training von KI und Spracherkennung sowie die Verbesserung der Sprachsynthese sind Forschungsaufgaben, mit denen wir uns auseinandersetzen. Hier können wir bereits auf Erfahrungen und Methoden zurückliegender Projekte zur automatisierten Erkennung von Markern der Gesprächsqualität zurückgreifen (vgl. Walther 2018; Meißner et al. 2011). Die grundlegende Frage nach dem sinnvollen und praktikablen Einsatz von KI auf der einen und der Unverzichtbarkeit individueller und hochqualifizierter zwischenmenschlicher Interaktion auf der anderen Seite bildet die Grundlage unserer Forschung und ist gegenstands- und formatbezogen immer wieder neu zu beantworten. 


\section{$1.2 \quad$ Audio-Optimierung}

Aus der Callcenter-Forschung ist bekannt, dass die Arbeit am Telefon für die Mitarbeiter*innen eine hohe Belastung darstellt. Zunächst auf stimmlicher Ebene: Wer den ganzen Tag am Telefon sprechen muss und sich dabei ggf. noch von anderen Sprechenden oder von Umgebungsgeräuschen abheben muss, erleidet nicht selten eine krankhafte Stimmstörung (vgl. Pietschmann 2017; Gebhardt/Becker 2007; Sportelli/Raestrup 2001). Neben Stimmtrainings, in denen die Callcenter-Agent ${ }^{\star}$ innen lernen, wie sie ihre Stimmen schonend nutzen, können mittlerweile auch technische Lösungen genutzt werden. Eine dieser Lösungen ist sonocom, das von den beiden Entwicklern Dr. Mario Götz und Johannes Raschpichler im Rahmen des Podiumsgesprächs vorgestellt wurde.

Mithilfe des sonocom-Systems kann der Klang der Mitarbeiter*innen, wie er schließlich bei den Anrufenden ankommt, im Bereich verschiedener akustischer Parameter verändert werden:

- Die Amplitude kann verstärkt werden, so dass die Stimme lauter klingt.

- Hintergrundgeräusche können herausgefiltert werden. Die Agent*innen bekommen diese Filterung live über Kopfhörer rückgekoppelt. Dadurch entsteht für sie der Eindruck, in einer leisen Umgebung zu sprechen und sie haben keinen Grund mehr, laut sprechen zu müssen.

- Der Stimmklang der Mitarbeiter*innen kann individuell verändert werden, so dass ihre Stimme beispielweise tiefer klingen und dadurch kompetenter wirken oder aber dass eine Stimme erhöht wird und einen leichten Hauch erhält, um einen freundlicheren Ausdruck zu bekommen (auch in Hinblick auf das sog. voice branding vgl. Lehmann 2008).

Neben der stimmlichen spielt auch die psychische Belastung eine nicht zu unterschätzende Rolle im Arbeitsalltag von Callcenteragent*innen (vgl. Gebhardt/ Becker 2007). Sie müssen sich innerhalb kürzester Zeit auf verschiedene Ge- 
sprächspartner*innen einstellen und sind oft die ersten, die die negativen Gefühle von Anrufer*innen abbekommen. Auch hierfür bietet sonocom Hilfen an:

- Durch die oben bereits erwähnte Unterdrückung von Umgebungsgeräuschen, die über das Headset rückgekoppelt wird, kann verhindert werden, dass Agent*innen aufgrund der Lärmbelastung Stress empfinden.

- Ein eingebauter Gehörschutz steuert automatisch den eingehenden Ton aus, so dass alle Geräusche automatisch in einer angenehmen und gesunden Lautstärke über das Headset zu hören sind.

- Die Mitarbeiterinnen können eine individuell auswählbare Hintergrundmusik festlegen. Diese Musik ist nur für die Agent ${ }^{\star}$ innen hörbar und kann helfen, deren Stimmung zu verbessern.

Ansatzpunkte für eine sprechwissenschaftliche Forschung wären hier zum einen zu evaluieren, ab wann die akustische Filterung den Kommunikationsprozess beeinträchtigt. So enthalten beispielsweise Hintergrundgeräusche aus rhetorisch-kommunikativer Perspektive Kontextinformationen. Wenn diese Geräusche zu stark gefiltert werden, könnte es erschwert werden, den situativen Kontext der Gesprächspartnerin bzw. des Gesprächspartners einzuschätzen und angemessen darauf zu reagieren, z. B. durch langsameres Sprechen oder gezielte Sprechpausen.

Zum anderen ist es interessant zu prüfen, ob die Technologie auch in anderen Forschungs- und Anwendungsgebieten der Sprechwissenschaft gewinnbringend eingesetzt werden kann. Denkbar wäre beispielsweise, dass das Sprechen von Menschen mit Sprech- oder Stimmstörungen in Echtzeit verbessert wird und dadurch deren kommunikative Teilhabe erhöht oder überhaupt erst wieder ermöglicht wird. 


\section{Unterstützung menschlicher Kommunikation durch Computer}

\section{1 „Argumentierende Suchmaschinen“}

Eine Vision, die nicht nur für die telefonische Kund ${ }^{*}$ innendienstleistung vielversprechend erscheint, zielt auf die rhetorisch-kommunikative Echtzeit-Unterstützung in der Live-Interaktion durch die IT. So könnten beispielsweise Mitarbeiter*innen im telefonischen und im omnichannel Kund ${ }^{*}$ innendienst gezielt mit Informationen und Hinweisen zur Gesprächsführung und sogar mit für die Gesprächspartner ${ }^{\star}$ innen passgenauen Argumenten im ,live-Gespräch unterstützt werden. Einen Grundstein für solche zukunftsweisenden Szenarien legt ein Projekt des Professors für Big Data-Analytics Dr. Mathias Hagen am Institut für Informatik der Martin-Luther-Universität Halle-Wittenberg. Mit Args.me (https://www.args.me/) entwickelte er die erste Websuchmaschine für Argumente (vgl. Internetquelle 5).

Anders als bei Suchanfragen via Google stützen sich die ,Treffer ' bei der Suche mit Args.me auf die Analyse großer Datenmengen, die durch Diskussionen in Internetforen öffentlich zugänglich sind. Typischerweise werden in solchen Foren Fragen und Probleme von User*innen diskursiv erörtert. Internetforen bieten damit ein differenziertes Abbild der öffentlichen Meinung und zugleich legen sie typische Denk- und Argumentationsmuster der Foren-User*innen und damit ein bestimmtes „[...] Verständnis von Ausschnitten der Welt“ (Hannken-Illjes 2018, 11) offen. Im Ergebnis liefert Args.me ein differenziertes Bild unterschiedlicher Pro- und Contra-Argumente, die eine bestimmte Lösung präferieren und diese zugleich argumentativ stützen.

Noch bedarf es einer konkreten Frage, die der ,Maschine über die Suchmaske gestellt, also per ,Hand` bzw. Tastatur eingegeben wird. Denkbar sind jedoch Szenarien, und hier soll nach Meinung von Prof. Hagen die Reise in der Zukunft auch hingehen, in denen Argumentationsbedarf z. B. im (Kund ${ }^{*}$ innen-)Gespräch in Echtzeit (via Spracherkennung) erkannt und mit Argumenten unterstützt wird.

Nimmt man die angestrebte automatisierte Erkennung des Argumentationsbedarfs zur Grundlage, geraten unweigerlich auch Fragen in den Blick, die 
auf die Passgenauigkeit von Argumenten abzielen. D. h., wenn über Spracherkennung und -analyse ein Begründungsbedarf erkannt wird, ist auch denkbar, dass die Sprachanalyse nicht nur den Bedarf an sich, sondern auch die kritische Fragestellung und die damit verbundenen möglichen Zweifel (sofern diese geäußert werden) erkennt. Denkbar sind also Big Data generierte Pro- und Contra-Argumente, die nicht nur das Strittige an sich, sondern das ,Verständnis der Welt' der jeweiligen Gesprächspartnerinnen offenlegen und damit passgenaue Argumentationen möglich machen.

Forschungsfragen und -gegenstände, die sich in diesem Zusammenhang für unser Fach ergeben sind:

- Welche sprachlichen Formen nutzen Gesprächspartner*innen zur Dissensmarkierung (als Grundlage für die automatische Erkennung)?

- Welche parasprachlichen Marker bzw. Komplexe typischer Merkmalsbündel mit spezifischer Ausprägung nutzen Gesprächspartner*innen um Dissens anzuzeigen?

- Aus welchen Bereichen (Topoi) beziehen Gesprächspartner*innen ihre Argumente?

- Lassen sich möglicherweise argumentative ,Grundmuster ${ }^{`}$ oder gar ,Grundtypen' ableiten und beschreiben?

Über Big-Data-Analysen lassen sich insbesondere für die sprechwissenschaftliche Rhetorikforschung wichtige Fragestellungen untersuchen und ggf. beantworten, die wohl auch in gesellschaftskritischer und ethischer Hinsicht von Belang sein dürften: Welche, möglicherweise auch enge, eingeschränkte oder ausgrenzende, Sicht auf die Welt und die Gültigkeit von Argumenten wird offenbar und wie gehen wir eigentlich im Geben und Nehmen von Gründen miteinander um?

\section{2 „Sprechende Wörterbücher“}

Nachschlagewerke, auf die über das Internet zugegriffen werden kann, sind beliebt und laufen den meisten ihrer Äquivalente in Buchform den Rang ab. Wikipedia ersetzte als freie Internet-Enzyklopädie in den letzten 20 Jahren 
(Gründung im Januar 2001) mehr und mehr den Griff zum herkömmlichen Lexikon, wenn es darum geht, sich kurz über ein Stichwort zu informieren. Seit 2011 sind alle Informationen aus dem Rechtschreib-Duden kostenlos über das Internet erreichbar und auch zweisprachige Wörterbuch-Angebote zu den meisten Sprachen der Welt sind online frei zugänglich. Ebenfalls in die Reihe der Nachschlagewerke, zu denen Ergänzungen oder Alternativen im Web entwickelt werden, gehören Aussprachewörterbücher. Für diese ergeben sich durch die Verfügbarmachung im Internet gleich mehrere Vorteile, wie dem Beitrag von Ebel, Hientz, Hirschfeld und Skoczek in diesem Band entnommen werden kann.

Insbesondere die Möglichkeit, zu jedem Lemma die passende Audiodatei zur Verfügung zu stellen, damit nicht nur die Transkription eines Wortes gelesen, sondern die Aussprache direkt angehört werden kann, bietet einen enormen Mehrwert für Nutzer*innen, da nicht jede ${ }^{\star}$ r in der Lage ist, die phonetische Umschrift korrekt zu lesen.

Sofern der Anspruch besteht, dass die Audioaufnahmen die jeweils abzubildende Transkription eineindeutig widergeben und dass eine einzige Stimme alle Einträge vertont, sollte auf Sprachsynthese zurückgegriffen werden. Die Anforderungen an ein solches Text-to-Speech-System (TTS-System) sind im Kontext eines zu vertonenden Aussprachewörterbuchs allerdings relativ hoch:

- Die Verständlichkeit muss nicht nur auf der Wortebene gewährleistet sein, sondern bis auf die Ebene der einzelnen Laute exakt stimmen. Merkmale wie Plosivaspirationen oder Entstimmlichungen sollten ebenfalls hörbar sein. Das Auftreten solcher Merkmale folgt in der Standardaussprache einer Systematik, so dass ein TTS-System entsprechend angelernt oder programmiert werden könnte.

- Die Prosodie spielt insbesondere auf Wortakzentebene eine wichtige Rolle. Oft scheitern Sprachsynthesesysteme daran, dass Haupt- und Nebenakzente gleich stark realisiert werden oder zwischen den verschiedenen Abstufungen der unakzentuierten Silben nicht ausreichend differenziert wird, wodurch der Klang der synthetisierten Wörter an Natürlichkeit einbüßt. Des Weiteren hat die Melodie einen Einfluss darauf, ob ein Wort als natürlich wahrgenommen wird oder nicht. 
Für die Deutsche Aussprachedatenbank, die in der Abteilung Sprechwissenschaft und Phonetik entwickelt wird, wird aktuell noch an einer praktikablen Lösung für die Synthese der 130.000 Einträge gesucht. Die großen Fortschritte in der Weiterentwicklung der TTS-Systeme innerhalb der letzten Jahre stimmen aber optimistisch, dass bald ein System gefunden wird, dass die Anforderungen erfüllen kann.

\section{Digitalisierung der Lehre}

\subsection{Mixed Reality unterstütztes Stimmtraining}

Stimmtraining und Sprechbildung sind Teile des Sprechwissenschaftsstudiums. Sie dienen jedoch nicht nur der Ausbildung eigener Kompetenzen, sondern zielen auch auf die Didaktisierung ab. Denn in sprechintensiven Berufen hilft gezieltes Training, die Stimmlippen vor dauerhafter Überlastung und somit vor irreversiblen Schäden zu schützen. Oft findet Stimmtraining in kleinen Gruppen, bestenfalls sogar als Einzelunterricht statt, so dass individuell geübt werden kann. Das bringt jedoch mit sich, dass das Training in kleinen Räumen stattfindet. Die Aufgabe, in großen Räumen mit Umgebungsgeräuschen oder einem konstanten ,Lärmteppich` zu sprechen, kann also nicht oder nur selten unter realen Bedingungen geübt werden. Und selbst wenn es den Übenden gelingt, sich einen großen Hörsaal vorzustellen, dann fehlt noch immer die auditive Rückkopplung, die sich aus den Halleigenschaften des konkreten Raumes ergeben würde.

An einer praktikablen Lösung haben Wissenschaftler*innen der RWTH Aachen im Rahmen des BMBF-geförderten Projekts ELLI zusammen mit der Abteilung Sprechwissenschaft des Instituts für Sprach- und Kommunikationswissenschaft der RWTH Aachen gearbeitet. Unter ihnen auch der Sprechwissenschaftler Björn Meißner, der als Podiumsgast an der Diskussion beteiligt war und zusammen mit seiner Kollegin Kathrin Hohlbaum ein technisches System zum Mixed Reality unterstützten Stimmtraining für Lehrende vorstellte: Mithilfe einer VR-Brille und Kopfhörern kann der Eindruck simuliert werden, sich in einem ausgewählten Hörsaal zu befinden. Wenn man nun in ein 
angeschlossenes Mikrofon spricht, erhält man über Kopfhörer die Rückkopplung, wie die eigene Stimme unter den konkreten Raumeigenschaften klingen würde, wodurch das Üben erleichtert wird. Durch diese Kombination realer Stimmtrainings mit einer virtuellen Umgebung wird das Üben in geschützten und dennoch realitätsnahen Umgebungen ermöglicht. So haben Lehramtsstudierende und Lehrende die Möglichkeit, erlernte Techniken für einen physiologischen und wirkungsvollen Stimmeinsatz in einer solchen Umgebung auszuprobieren, zu erleben und zu variieren.

Für den Einsatz von Mixed Reality unterstütztem Stimmtraining sind mit Hilfe von erweiterter Software auch noch Feedback-Funktionen denkbar, die dem*der Übenden z. B. direkt zurückmelden, ob die Stimme auch auf allen Sitzplätzen im Raum hörbar war. Auch eine Echtzeit-Auswertung von phonetischen Parametern wie der Sprechgeschwindigkeit oder des Grundfrequenzverlaufs sind denkbar, damit Übende den situativ angemessenen Einsatz von Tempo- und Melodievariationen üben könnten.

\subsection{Lernvideos}

Lernvideos und Tutorials auf YouTube werden immer beliebter und es gibt mittlerweile fast nichts, wozu man keine Erklärung in Videoform finden kann. Die Gründe, die für das Lernen mit YouTube sprechen, sind Unabhängigkeit von Ort und Zeit, eine breite Themenvielfalt sowie die multisensorische Darstellung der Inhalte. Diese Vorteile wissen auch Schüler*innen und Studierende zu schätzen, die ihre ,Nachhilfestunde' dann wahrnehmen können, wenn sie sie brauchen und dafür bereit sind.

Auf die gesteigerte Nachfrage nach Lernvideos reagieren daher auch Lehrkräfte und Hochschuldozent ${ }^{\star}$ innen. ,Lehrerschmidt' begann zum Beispiel Videos als Ergänzung zu seinem Mathe- und Physikunterricht auf YouTube zu veröffentlichen (Perfler 2020) und ein Professor für Mathematik und Statistik erklärt anhand anschaulicher Beispiele statistische Grundbegriffe (vgl. Internetquelle 6). An der Martin-Luther-Universität Halle-Wittenberg sollen in einem Projekt des Zentrums für Lehrer*innenbildung namens „Körper - Stimme - Haltung | Wirkungsstrategien für Lehrer*innen“ Videos zum Weiterüben angeboten werden (vgl. Internetquelle 7). Und im Projekt „[D-3] 
Deutsch Didaktik Digital“ am Germanistischen Institut steht der Einsatz von Lernvideos für zukünftige Lehrer*innen im Zentrum (vgl. Internetquelle 8). Zur Podiumsdiskussion hatten wir Mirko Drotschmann eingeladen, der seit 2012 als YouTuber MrWissen2go sehr erfolgreich Videos zu geschichtlichen und politischen Unterrichtsthemen veröffentlicht.

Die Erforschung von Lernvideos auf YouTube ist aus verschiedenen Gründen auch aus sprechwissenschaftlicher Sicht bedeutsam (vgl. Ebel 2018):

- Medial vermitteltes Sprechen ist eines der originären Forschungsobjekte der Sprechwissenschaft. Sprechwirkungsanalysen können Hinweise darauf geben, welche Sprechausdrucksmerkmale zu besserer Verständlichkeit der Lerninhalte führen können (vgl. Ebel 2021).

- Die rhetorische Gestaltung der Videos ist unter anderem deshalb interessant, weil sie oft einen doppelten Zweck verfolgt: Neben der Vermittlung der Lerninhalte geht es bei YouTube-Videos zumeist auch um Erfolg, dargestellt in Form von Klickzahlen, Likes, Abonnements und Kommentaren (vgl. Ebel 2020, 4).

Die Covid-19-Pandemie und die damit verbundenen Einschränkungen des Alltags haben zu einem sprunghaften Anstieg der digitalen Lehre geführt, der bei unserer Podiumsdiskussion im Januar 2020 noch überhaupt nicht abzusehen war. Der Bedarf an guten Lernvideos und anderen internetbasierten Lehrformen ist wichtiger denn je und die Sprechwissenschaft sollte ihren Teil zur Verständnissicherung beitragen.

\section{Digitalisierung im medizinischen Bereich}

\subsection{Translationsregion für digitalisierte Gesundheitsversorgung (TDG) - ein Modellprojekt an der MLU}

Das Modellprojekt Translationsregion für digitalisierte Gesundheitsversorgung (TDG) der Medizin und Pflegewissenschaft der Martin-Luther-Universität Halle-Wittenberg ist ein regionales Strukturwandelprogramm, das auf die 
Verbesserung der medizinisch-pflegerischen Versorgung und die Teilhabeförderung alter und pflegebedürftiger Menschen in strukturschwachen Regionen Mitteldeutschlands abzielt. Angesiedelt ist das TDG-Projekt am Bundesministerium für Bildung und Forschung (BMBF) im Rahmenprogramm WIR! (Wandel durch Innovation in der Region). Das Projektbündnis beabsichtigt, einen umfassenden Innovationsprozess in der Pflege- und Gesundheitswirtschaft anzustoßen - von der Idee bis zur Pilotierung und Umsetzung (https:// inno-tdg.de/). Die TDG hält Fördermittel zur Umsetzung von Projektideen bereit, die durch innovative Digitalisierungskonzepte einen Beitrag leisten in den drei Handlungsfeldern (vgl. TDG-Report 2018, 9):

- digitale wohnortnahe Versorgungskonzepte,

- teilhabeförderliche digitalisierte Wohnformen bei Pflegebedürftigkeit,

- digitale Qualifizierungskonzepte für Fachkräfte und Angehörige.

$\mathrm{Da}$ alle Bereiche nicht denkbar sind ohne Interaktion, sehen wir auch hier einen Bezug zur Sprechwissenschaft. Szenarien, um Pflege, medizinische Versorgung aber auch Teilhabe via Digitalisierung zu gewährleisten, hat Dr. Karsten Schwarz, Mitarbeiter im TDG-Konsortium, anlässlich der Podiumsdiskussion zu den ,Kleinen-Fächer-Wochen' dargestellt, darunter den Pflege-Roboter ,Pepper' (vgl. Internetquelle 9).

Der 1,20 Meter große Roboter soll u. a. mit Menschen interagieren. Er versteht einfache Sätze, gibt Sprache wieder und kann Gesichter erkennen, sich merken und sogar mit dem Blick folgen. Neben der Frage, wie und wo Pepper in der Pflege noch sinnvoll eingesetzt werden kann, beteiligen sich Pflegewissenschaften, Wirtschaftsinformatik und Mediziner an der grundlegenden Diskussion darüber, wie der Pflegeroboter überhaupt mit Menschen in Interaktion kommen kann und wie ganz allgemein mit Hemmnissen und Vorbehalten gegenüber technischen Lösungen umgegangen werden kann. „Dabei steht für die Forscher schon mal fest: Einen Menschen kann und soll Pepper nicht ersetzen." (vgl. Internetquelle 9).

In Bezug auf sinnvolle Einsatzfelder des Pflegeroboters ist beispielsweise angedacht, dass Pepper bei einem MRT-Termin im Krankenhaus oder der Pra- 
xis Patient*innen vorbereitet und den Untersuchungsablauf erklärt. Denkbar ist auch, dass Pepper das Reha-Training unterstützt, den Patient ${ }^{*}$ innen Übungen zeigt und sie zum Mit- und Nachmachen animiert (vgl. ebd.).

In der grundlegenden Diskussion über die interaktiven Möglichkeiten und Grenzen und die Annahmebereitschaft des Pflegeroboters, aber auch anderer digitaler Assistenzsysteme, sehen wir eine zentrale Schnittmenge zu unserem Fach. Fragestellungen, denen nachgegangen werden sollte, sind u. a.:

- Wie müssen Roboter und Avatare kommunikativ agieren, um akzeptiert und angenommen zu werden?

- Wie künstlich oder echt sollte das Sprechen gestaltet sein?

- Wie ,aufrichtig' sollte auch Nicht-Können von Maschinen angezeigt werden?

- Was sind sinnvolle Anwendungsgebiete sprechender Maschinen, Avatare und Roboter in der Pflege und therapeutisch-medizinischen Versorgung?

Antwort auf die letzte der Fragen gibt ein aktuelles Forschungsprojekt der Sprechwissenschaft, das die Digitalisierung der Aphasietherapie zum Ziel hat und über das nachfolgend berichtet wird.

\section{2 aphaDIGITAL -} ein Projekt zur Digitalisierung der Aphasietherapie

Dass die Digitalisierung auch in der sprachtherapeutischen Versorgung dringend notwendig ist, wird unter den aktuellen Corona-Bedingungen mehr als deutlich. Logopäd ${ }^{*}$ innen und Sprachtherapeut ${ }^{*}$ innen fordern eine systematische Konzeption sprachtherapeutischer Teletherapie, bei der neben inhaltlichen Rahmenbedingungen v. a. auch technische und datenschutzrechtliche Anforderungen und Standards definiert werden (vgl. Bilda et al. 2020, 176). Das digitale Angebot, insbesondere in Form von Apps und Softwareprogrammen, ist zwar inzwischen breit aufgestellt, jedoch von einer systematischen digitalen Versorgungskonzeption weit entfernt. 
Mit dem Projekt aphaDIGITAL setzen wir an diesen Missständen der sprachtherapeutischen Versorgungssituation in Deutschland, insbesondere in Mitteldeutschland, an. Das Projekt ist eingebunden in das unter Kap. 4.1 beschriebene TDG-Bündnisprojekt. Die von den eingangs zitierten Autor*innen beschriebene "digitale Transformation der Gesundheitsversorgung" (ebd.) scheint imstande, die grundlegenden Versorgungslücken zu schließen, unter denen Patient ${ }^{\star}$ innen und Therapeut ${ }^{\star}$ innen vielerorts schon seit geraumer Zeit leiden: Der allseits beklagte Fachkräftemangel, eine ausgesprochen schlechte Versorgungssituation gerade in ländlichen und strukturschwachen Regionen, lange Anfahrtswege und Fahrzeiten, geringe Therapiefrequenz und die durch den demografischen Wandel stets steigende Patient*innenzahl sind Defizite, welche die sprachtherapeutische Versorgung derzeit beschreiben. Gerade im Bereich der neurologischen Störungen und insbesondere dem Störungsbild der Aphasie führen die genannten Faktoren mittel- und langfristig zu einer Gefährdung des Therapieerfolgs.

Mit Blick auf das bestehende, zumeist therapie-unterstützende digitale Angebot für die Aphasie wird deutlich, dass dieses nicht ganzheitlich und den ICF-Kriterien gemäß konzipiert ist. Neben der Sprechwissenschaft besteht das Projektteam aus Mitarbeitern der Wirtschaftsinformatik der TH-Wildau, einem IT-Partner aus dem Bereich App- und Spieleentwicklung sowie aus Praxispartnern der Logopädie und Sprachtherapie, darunter auch die Logopädieschule des Universitätsklinikums in Halle (UKH).

Das digitale Setting von aphaDIGITAL und seinen Komponenten für das Eigentraining via App (aphaTRIA) und die Teletherapie (aphaTHERA) strebt eine 'all-in-one'-Lösung an, die flächendeckend für die Aphasietherapie genutzt und schrittweise auf andere Störungsbilder erweitert werden kann. Ziel ist, die durch Fachkräftemangel und Strukturwandel bedingte Versorgungslücke durch die Entwicklung digitaler Lösungen zu schließen.

Eine hochfrequente Übungsbehandlung ist nachgewiesenermaßen essenziell für den Therapieerfolg und fördert die kommunikative Teilhabe der Patient*innen. Die zu entwickelnden aphaDIGITAL-Lösungen sollen dazu beitragen, eine frühzeitige, hochfrequente und individuelle sprachtherapeutische Versorgung zu gewährleisten und damit langfristig den Therapieerfolg zu sichern. Das Projektvorhaben gliedert sich in zwei Phasen: 
1. aphaTRIA: Entwicklung eines avatargestützten Übungsszenariums (App) via Tablet

2. aphaTHERA: Entwicklung eines vollwertigen, multimodalen Teletherapie-Raumes zur dezentralen Versorgung

Der Avatar bildet die Kernidee des Projektes. Er soll das Eigentraining und häusliche Üben der Patient ${ }^{\star}$ innen in einem motivierenden und interaktiven Setting begleiten. Zugleich soll der Avatar mit minimal-therapeutische Funktionen, in Form von Anlauthilfen und Mundbild zur Unterstützung des Wortabrufes ausgestattet sein. Er führt interaktiv durch die Übungssessions und wird zumzur virtuellen Übungsbegleiter*in. Die Daten zu Übungsfrequenz und -verlauf stehen als motivierendes Patient*innenfeedback zur Verfügung und dienen dem $^{\star}$ der Sprachtherapeut ${ }^{\star}$ in kontinuierlich zur Einschätzung des Therapieerfolges und Planung des weiteren Verlaufs. Der*die Therapeut*in ist also stets eingebunden in das häusliche Üben, kann Übungsstatistiken abrufen, Übungen und Übungsmaterial individuell zusammenstellen und so Übungsinhalte und Schwierigkeitsstufen auf den ${ }^{\star}$ die Patient ${ }^{\star}$ in anpassen. Die triadische Beziehung aus Patient ${ }^{\star}$ in, Therapeut ${ }^{\star}$ in und dem digitalen Setting (App mit Avatar und Tablet) sichert eine therapeutisch begleitete Übungsumgebung und soll später um eine Tele-Therapie-Komponente erweitert werden.

Forschungsfragen, die sich aus diesem Projekt ergeben, reichen von der interaktiven Rahmung, dem entsprechenden Interaktionsvokabular des Avatars, über grundlegende Aspekte der Avatar-Modellierung (auch in Bezug auf den Uncanny-Valley-Effekt vgl. MacDormann 2019) bis hin zu der Frage nach geeigneten KI-Lösungen für die Sprach- und Mimikerkennung sowie der grundlegenden Frage nach den Grenzen von virtueller Übungsbegleitung in der Tele-Therapie:

- Welche angemessenen Feedback-Reaktionen kann und sollte der Avatar ,können` im Spannungsfeld zwischen Beobachtung und Bewertung?

- Sollte der Grad der Entfremdung an das ,Können' des Avatars angepasst sein?

- In welchem Umfang sollte der Avatar individualisierbar sein? 
- Was sollte er nicht können oder zu können suggerieren und sollte dieses Nicht-Können explizit gemacht werden?

- Ist eine Kopplung von Sprach- und Mimikerkennung sinnvoll?

- Wie müssen die Übungen zur Sprachproduktion , phonetisch konzipiert sein?

- Wo liegen die technischen und therapeutischen Grenzen von Teletherapie?

\section{$5 \quad$ Fazit}

Nicht nur dieser Beitrag, auch die jüngsten Entwicklungen in Zusammenhang mit der Covid-19-Pandemie zeigen, dass Digitalisierung von und in der Kommunikation in vielfältiger Art und Weise Teilhabe ermöglicht und fördert, sinnvolle Unterstützung leistet und auch für die Zukunft Szenarien entwirft, das Miteinandersprechen gezielt zu unterstützen und zu gestalten. Die Sprechwissenschaft steht hier in der Pflicht, Entwicklungen, Wege und Visionen wissenschaftlich zu begleiten, aber auch kritisch in Frage zu stellen. Gesellschaftliche Kommunikation und Teilhabe sind seit jeher im Wandel, spielen jedoch eine weiter zunehmende Rolle. Aufgabe unseres Faches, welches das Miteinandersprechen in den Kern des Erkenntnisinteresses setzt, muss es deshalb sein, diesen Wandel zu begleiten und durch Theoriebildung, Forschung und Didaktik zu begleiten.

Die Frage nach dem sinnvollen Einsatz, Unterstützung und ggf. auch Ersatz zwischenmenschlicher Kommunikation durch digitale Lösungen wirft zugleich die Frage nach den Bereichen und Kontexten auf, in denen zwischenmenschliche Interaktion unverzichtbarer und durch nichts zu ersetzender Bestandteil gesellschaftlicher Praxis ist und bleiben muss. 


\section{Literatur}

Bilda, Kerstin/Dörr, Fiona/Urban Kjara/Tschuscke, Benjamin (2020): Digitale logopädische Therapie. Ergebnisse einer Befragung zum aktuellen Ist-Stand aus Sicht von LogopädInnen. In: Logos. Die Fachzeitschrift für akademische Sprachtherapie und Logopädie. 3/2020, S. 176-183.

Ebel, Alexandra (2018): Lehr-Lern-Videos auf YouTube als sprechwissenschaftlicher Analysegegenstand. In: sprechen, Heft 65, S. 5-11.

Ebel, Alexandra (2020): Was passiert mit sprecherischen Elementarprozessen in Zeiten von Social Media? Ein Problemaufriss am Beispiel von YouTube-Videos. In: Kranich, Wieland (Hrsg.): Sprechwissenschaft heute. Baltmannsweiler: Schneider, S. 1-8.

Ebel, Alexandra (2021): Einfluss sprachlicher und sprecherischer Merkmale auf die Verständlichkeit von Lernvideos auf YouTube. In: Staubach Katharina (Hrsg.): Multimodale Kommunikation in den Hypermedien. Theoretische, empirische und unterrichtspraktische Zugänge. Baltmannsweiler: Schneider, S. 250-275.

Gebhardt, Raimand/Becker, Mario (2007): Die Stimme als Kapital im Call-Center. In: Profil Concept I/2007 https://www.profil-concept.net/images/profilconcept/ PDFs/Einblick-ProfCon-Stimme_als_Kapital_im_Call-Center-2007_01.pdf (letzter Zugriff: 2020-11-21)

Hannken-Illjes, Kati (2018): Argumentation. Eine Einführung in die Theorie und Analyse der Argumentation. Tübingen: Narr Francke Attempo

Lehmann, Mark (2008): Voice Branding. Die Stimme in der Markenkommunikation. Reihe: Praxisforum Medienmanagement Bd. 10, München: Verlag Reinhard Fischer.

MacDorman, Karl F. (2019): In the uncanny valley, transportation predicts narrative enjoyment more than empathy, but only for the tragic hero. In: Computers in Human Behavior 94, S. 140-153.

Meißner, Swetlana/Pietschmann, Judith/Walther, Mathias/Nöbel, Lars (2011): Innovative IT-gestützte Ansätze zur Bewertung der Gesprächsqualität in Telefonverkaufsgesprächen. In: Hirschfeld, Ursula/Neuber, Baldur (Hrsg.): Erforschung und Optimierung der Callcenterkommunikation. Berlin: Frank \& Timme, S. 195-213.

Perfler, Gerald (2020): Lehrerschmidt - Einfach lernen! In: Tibs. Tiroler Bildungsservice: https://tibs.at/content/lehrerschmidt-einfach-lernen (letzter Zugriff: 2020-10-20)

Pietschmann, Judith (2017a): Forschungsbasiertes Qualitätsmanagement im Callcenter - Ein Weg von der Empirie in die Praxis. In: Neuber, Baldur/Pietschmann, Judith (Hrsg.): Dialogoptimierung in der Telekommunikation. Berlin: Frank \& Timme, S. 41-82.

Pietschmann, Judith (2017b): Optimierung von Gesprächen in der professionellen Telefonie. Berlin: Frank \& Timme. 
Walther, Mathias (2018): Automatische Erkennung paralinguistischer Merkmale zur Bewertung der Gesprächsqualität in Callcentern. Reihe: Studientexte zur Sprachkommunikation Bd. 89, Dresden: TUDpress.

Sportelli, Anja/Raestrup, Bernhard (2001): Call-Center als Sprechberuf -Belastungsfaktoren und Stimmerkrankungen. CCall-Report 2.

TDG-Report (2018): https://inno-tdg.de/wp-content/uploads/2019/02/TDG_ REPORT_web.pdf

\section{Internetquellen}

Internetquelle 1: https://callcenter-verband.de/kein-ersatz-fuer-persoenlichen-kontakt/ (letzter Zugriff 24.02.2021)

Internetquelle 2: https://ai.googleblog.com/2018/05/duplex-ai-system-for-naturalconversation.html (letzter Zugriff 21.11.2020)

Internetquelle 3: https://callcenter-verband.de/ (letzter Zugriff 24.02.2021)

Internetquelle 4: https://www.genesys.com/de-de/blog/post/fragen-manfredstockmann-prasident-des-call-center-verband-deutschland-e-v (letzter Zugriff 24.02.2021)

Internetquelle 5: https://www.informatik.uni-halle.de/arbeitsgruppen/big_data_ analytics/forschung/ (letzter Zugriff 24.02.2021)

Internetquelle 6: https://www.youtube.com/channel/UCtBEklAtHHji2V1TsaTzZXw/ about (letzter Zugriff 21.11.2020)

Internetquelle 7: https://www.zlb.uni-halle.de/zentrum/projekte/koerper_stimme_ haltung/ (letzter Zugriff: 24.02.2021)

Internetquelle 8: https://d-3.germanistik.uni-halle.de/sitzung/lehr-lernvideos/ (letzter Zugriff: 24.02.2021)

Internetquelle 9: https://www.mdr.de/wissen/pepper-roboter-pflege-100.html (letzter Zugriff: 24.02.2021) 

Susanne Vollberg, Halle (SaAle)

\section{Von der Suche nach dem Glück oder „Das ideale Ich“. Selbstoptimierung im Netz}

Zusammenfassung: Was ist Glück? Kann man das Glück messen und damit auch beeinflussen? Auf Grundlage der Glücksforschung unterschiedlicher wissenschaftlicher Disziplinen setzt sich der Aufsatz mit den modernen Vorstellungen eines glücklichen Lebens und den vielfältigen Selbstoptimierungsbestrebungen im Web 2.0 auseinander.

Schlagwörter: Glück, Selbstoptimierung, Welt-Glücksreport, Gesundheit, Glücksforschung

Abstract: What is happiness? Can happiness be measured and thus influenced? Based on the happiness research of different scientific disciplines, the essay deals with the modern ideas of a happy life and the various self-optimization efforts in Web 2.0.

Keywords: Happiness, Quantified-Self, Word-Happiness-Report, Health, Happiness Research

"We all seem to be looking for happiness - but perhaps we are looking in the wrong places. We have gotten richer as societies but often failed to become happier." (Internetquelle 1)

Im Wintersemester 2019/2020 veranstaltete das Institut für Musik, Medienund Sprechwissenschaften (IMMS) an der Martin-Luther-Universität Halle-Wittenberg die öffentliche Ringvorlesung Ideale in Musik, Gesang, Medien, Sprache und Sprechen im Rahmen der „Kleine Fächer Wochen Sprechwissenschaft“. Dieser Beitrag basiert auf der am 21. November 2019 gehaltenen gleichnamigen Vorlesung der Autorin. Auf Grundlage der Glücksforschung unterschiedlicher wissenschaftlicher Disziplinen setzt sich der Aufsatz mit 
den modernen Vorstellungen eines glücklichen Lebens und den vielfältigen Selbstoptimierungsbestrebungen im Web 2.0 auseinander.

\section{Fragen zum Auftakt: Was macht glücklich?}

Die Frage nach dem Glück ist von so grundsätzlicher Bedeutung für den Menschen, dass sie immer wieder und in immer neuen Zusammenhängen gestellt wird. Die Literatur über das erhabene Gefühl des Glücks oder die unendliche Suche nach dem ewigen Glück füllt Bibliotheken seit Jahrhunderten. In Filmen und Serien können wir tagtäglich die unterschiedlichsten Protagonist*innen auf ihrer oftmals komplizierten Suche nach dem Glück begleiten und am Ende wohlig ins Happy End versinken. Und wenn unsere Held*innen am Ende schon nicht das Glück gefunden haben, dann konnten sie doch wenigstens das große Unglück verhindern.

Das Streben nach einem glücklichen Leben oder die Suche nach dem Glück sind auch im Netz allgegenwärtig. Google zeigt auf die Frage Was ist Glück? ungefähr 137 Mio. Ergebnisse (0,51 Sekunden) und verweist auf weitere naheliegende Fragen unter der Rubrik Nutzer fragen auch: „Was ist Glück für mich?“ „Was ist Glück einfach erklärt?“ und „Was ist das Glück im Leben?“ Auf die Frage „Was macht glücklich?“ erhält man rund 66 Mio. Treffer. Und unzählige digitale Angebote - von der Happify-App bis zum youtube-Kanal MinisteriumFürGlück - versprechen Anleitungen für ein glücklicheres Leben. Jeder von uns, so die klare Botschaft, hat es selbst in der Hand, sein Leben zu verändern und damit glücklicher zu werden.

Der Mensch will sich selbst und seine Welt stets verbessern, um damit sein Wohlbefinden und das persönliche Glück zu steigern. (Balandis/Straub 2018, 131) Es scheint, als biete das Internet dieser anthropologischen Bestimmung viele neue Möglichkeiten, um das ideale Ich für ein glückliches Leben im Sinne einer Optimierung des eigenen Selbst zu formen. Ist also die Idee der Selbstoptimierung in Zeiten des Web 2.0 der moderne Weg zum Glück? 


\section{$2 \quad$ Was ist Glück?}

In der deutschen Sprache hat der Begriff Glück zwei Bedeutungen: Zum einen im Sinne des ,Glück haben' als Ergebnis des Zusammentreffens besonders günstiger Umstände. Zum anderen als Zustand des, glücklich sein` als temporäres Empfinden der inneren Befriedigung und Hochstimmung. Diese emotionale Befindlichkeit umfasst das Erleben eines positiven Gefühlszustandes und die Abwesenheit von negativen Empfindungen (vgl. Dallmer 2020, 41). Doch wie erreicht man den Zustand der Glückseligkeit? Die letztlich immer wiederkehrende Frage ist, ob Glück in Äußerlichkeiten (in Gegenständen, im Beruf, in einem anderen Menschen oder an einem bestimmten Ort) zu finden sei oder nur im Inneren durch das einzelne Individuum gefühlt werden kann. Aber was ist Glück dann überhaupt? Das Ideal der Selbstverwirklichung des Menschen? Eine Utopie? Nur eine Illusion?

Oder lässt sich die Frage nach dem Glück jenseits philosophischer Überlegungen vielleicht ganz einfach beantworten? Liefert der Internationale Tag des Glücks und der damit verbundene World Happiness Report die universelle Antwort? Genügt ein Besuch im dänischen Glücksmuseum ${ }^{1}$, um den Weg zum Glück zu verstehen?

Der Internationale Tag des Glücks (International Day of Happiness) existiert seit 2013 und wird am 20. März jeden Jahres gefeiert. Auf Initiative Bhutans beschlossen die Vereinten Nationen am 28. Juni 2012 in ihrer Resolution 66/281 die Einführung des Internationalen Tag des Glücks. (Internetquelle 2) Die Vereinten Nationen erkennen damit an, „wie bedeutsam Glück und Wohlbefinden als universelle Ziele und Bestrebungen im Leben der Menschen in aller Welt sind und wie wichtig ihre Anerkennung im Rahmen der Zielsetzungen der öffentlichen Politik ist“ (ebd.). Hervorgehoben wird außerdem die „Notwendigkeit eines inklusiveren, gerechteren und ausgewogeneren Konzepts für Wirtschaftswachstum, das die nachhaltige Entwicklung, die Armutsbeseiti-

1 Im Juli 2020 eröffnete das erste Glücksmuseum der Welt: The Happiness Museum in Dänemarks Hauptstadt Kopenhagen. Das Ziel? "We all seem to be looking for happiness - but perhaps we are looking in the wrong places. We have gotten richer as societies but often failed to become happier. Therefore, the Happiness Research Institute decided to create a museum where we can bring happiness to life." (Internetquelle 1) 
gung, das Glück und das Wohlbefinden aller Völker fördert“ (ebd.). Bereits ein Jahr zuvor, 2011, hatten die Vereinten Nationen „in der Erkenntnis, dass der Indikator Bruttoinlandsprodukt seinem Wesen nach nicht darauf angelegt ist, das Glück und das Wohlbefinden der Menschen in einem Land zu messen, und dass er sie auch nicht angemessen erfasst“ ihre Mitgliedsstaaten dazu aufgefordert „zusätzliche Maße zu entwickeln, die die Bedeutung des Strebens nach Glück und Wohlbefinden bei der Entwicklung besser erfassen, damit sie sich in ihrer nationalen Politik davon leiten lassen“. (Internetquelle 3)

Glück und Wohlbefinden werden damit zu zentralen Kategorien, die durch Regierungen ,erfasst ${ }^{`}$ und realisiert werden sollen. Doch wie misst man das Glück?

\subsection{Die Vermessung des Glücks}

Über Jahrhunderte haben Dichter*innen und Denker*innen über das Glück geschrieben. Heutzutage sind es Soziolog*innen, Ökonominnen und Marktforscher ${ }^{\star}$ innen, die per Onlinebefragung der Lebenszufriedenheit der Menschen nachspüren. Anstatt das Wohlbefinden eines Menschen oder einer ganzen Volkswirtschaft über ökonomische Kategorien wie Einkommen oder Wirtschaftsleistung abzubilden, werden Menschen direkt befragt, wie sie ihre aktuelle Lebenssituation bewerten. Damit haben Forscher*innen ein starkes Werkzeug in der Hand und die sogenannte „Glücksforschung“ boomt. Findet man in den Zahlen endlich eine Antwort auf die Frage: Was macht glücklich?

Große mediale Resonanz erfährt jedes Jahr der World Happiness Report. (Internetquelle 4) Der erste Report, der parallel zur Einführung des Weltglückstages erstellt wurde, zog international weite Beachtung auf sich. Er umfasste erstmals den Zustand des weltweit empfundenen Glücks und der Lebenszufriedenheit, die Gründe für Glück und Unglück sowie politische Folgerungen, die durch Fallstudien einzelner Länder belegt wurden. 2013 erschien der zweite World Happiness Report und seitdem wird, mit Ausnahme des Jahres 2014, jährlich ein neuer Report erstellt. Der Report vergleicht 156 Länder miteinander. Deren Bürgerinnen werden gefragt, wie glücklich sie sich selbst fühlen. Außerdem berücksichtigen die Forscher*innen Faktoren wie das Bruttoinlandsprodukt (BIP) pro Kopf, die Lebenserwartung und Korruption in Regierung 
und Wirtschaft. In den auswertenden Berichten beschreiben Expert*innen aus Wirtschaft, Psychologie, Umfrageanalyse und Statistik, wie die Ergebnisse effektiv genutzt werden können, um den Fortschritt von Nationen zu erfassen und Handlungsempfehlungen abzuleiten.

2019 stellte der „Weltglücksbericht“ zum zweiten Mal in Folge fest, dass in Finnland die glücklichsten Menschen leben. Dicht gefolgt von der Bevölkerung in Dänemark, Norwegen, Island und den Niederlanden. (Helliwell et. al 2019, 27) Das Glücksrezept der Finnen? Ein großartiges Land mit einer wunderschönen Natur. Ein sehr gutes Bildungssystem. Sicherheit. Und: „Wir wissen, wie man allein ist und auf seine eigenen Freiräume achtgibt“, „Wir haben unsere Saunas“, „Wir trinken am meisten Kaffee.“ (Internetquelle 5)

Weniger glücklich sind die Deutschen, sie erreichten zwischen 2016 und 2018 nur den 17. Platz im Ranking der glücklichsten Nationen und lagen damit beispielsweise noch hinter Costa Rica (Platz 12) oder Israel (Platz 13). (Helliwell et. al 2019, 27)

Neben dem World Happiness Report gibt es in Deutschland eine Vielzahl von Veröffentlichungen, die vergleichbare Daten aus Umfragen nutzen, um aufzuzeigen, wie es den Menschen jenseits von ökonomischen Größen wie Einkommen oder Wirtschaftswachstum geht. So misst der Deutsche Post Glücksatlas (Internetquelle 6) die Zufriedenheit der Deutschen nach Bundesländern und die Glücksstudie des SINUS-Instituts fand 2019 heraus: Der Schlüssel zum Glück ist Gesundheit. Für 51\% der Befragten stand die an erster Stelle, mit deutlichem Abstand werden genannt: Eine gute Partnerschaft (32\%), eine intakte Familie (31\%), ausreichend Geld (25\%) und ein schönes Zuhause (23\%). (Internetquelle 7)

In den Medien sind gerade diese interpersonellen, oft internationalen Vergleiche immer wieder ein beliebter Anlass zur Berichtserstattung. Seriöse Forscher*innen, die sich wissenschaftlich mit dem Glück auseinandersetzen, sehen die Aufarbeitung der vermeintlichen klaren Ergebnisse jedoch durchaus kritisch 


\subsection{Glücksforschung - Die Wissenschaft vom Glück?}

Populärwissenschaftlich aufgearbeitete Folgerungen aus den Zahlen der genannten Studien weisen zum Teil große methodische Schwächen auf, denn die Antwort auf die Frage nach der individuellen Lebenszufriedenheit ist immer ein „Besser/schlechter-Vergleich“. Bewertet ,Max Mustermann` seine Zufriedenheit am Montag mit 5 Punkten und am Mittwoch mit 10 Punkten, zeigt dies, dass er am Mittwoch zufriedener ist, nicht aber, dass er exakt doppelt so zufrieden ist. Noch komplexer wird der Vergleich der Befindlichkeit von Menschen aus verschiedenen Ländern. Hier unterscheiden sich die Einschätzungen zwischen Individuen und Kulturkreisen auffallend. Bewerten ,Max Mustermann' und ,John Doe ihre Zufriedenheit mit 5 Punkten, heißt das nicht, dass sie gleich zufrieden sind. Auch das Antwortverhalten ist in den einzelnen Kulturkreisen unterschiedlich. So neigen zum Beispiel Menschen des angelsächsischen Kulturkreises eher zu Extrem-Antworten als Asiaten, deren Antworten durchweg in der Mitte des Spektrums angesiedelt sind (Vgl. zu dieser Problematik zusammenfassend Dallmer 2020, S. 47-49).

Die wissenschaftlich-orientierte empirische Glücksforschung mit Wissenschaftlern wie Wolfgang Zapf oder Alfred Bellebaum fasste in Deutschland in den späten 1970er Jahren Fuß, deutlich beeinflusst von der US-amerikanischen Forschung. Heute ist die Glücksforschung eine etablierte Wissenschaft, die sich in viele Teildisziplinen auffächert (philosophische, physiologische, psychologische, ökonomische, experimentelle und sozialwissenschaftliche Glücksforschung). Ihr Gegenstand ist die Erforschung der Bedingungen, unter denen sich Menschen als glücklich bezeichnen, wann Menschen zufrieden sind und wie Wohlbefinden subjektiv wahrgenommen wird.

In wohlhabenden Gesellschaften ist, glücklich zu sein` zum zentralen Lebensziel der Bevölkerung geworden. „Menschen sind dann glücklich“, so der Magdeburger Soziologe Jan Delhey, Lehrstuhlinhaber für Allgemeine Soziologie und Makrosoziologie, „wenn sie ausreichend Geld zur Existenzsicherung haben, wenn sie gute soziale Kontakte zum Partner, zur Familie, zu Freunden haben und, wenn sie einen Sinn in ihrem Leben sehen." (Internetquelle 8). Diese Ergebnisse fasst Delhey in der Formel „Haben + Liebe + Sein = Glück“ (ebd.) zusammen. So einfach, so schwierig. Denn der Wunsch nach einem 
glücklichen Leben ist mit einer großen Unsicherheit verbunden, wie man dieses erreichen kann. Lebensformen und -verläufe sind für heutige Generationen viel offener und vielfältiger, damit aber auch weniger verbindlich als früher (ebd.).

\section{Selbstoptimierung = Alltägliche Wege zum Glück?}

Wenn Gesundheit und Wohlbefinden für die subjektive Wahrnehmung des Glücks von so elementarer Bedeutung sind, ist die Steigerung oder Festigung des eigenen Glücks dann nicht durch den Einzelnen recht einfach zu realisieren? Eröffnen die aktuellen Formen der Selbstoptimierung bzw. der Versuch, eine idealere Version des eigenen Ichs zu entwickeln, die Perspektiven zu einem zufriedeneren bzw. glücklicheren Leben?

Das Lexikon der Neurowissenschaft beschreibt Selbstoptimierung als grundlegende Eigenschaft des menschlichen Nervensystems. Sie kann „als spezielle Form des Lernens aufgefasst werden, da das Ergebnis eines ersten Versuchs einer bestimmten Handlungsweise die Vorgehensweise im nächsten Versuch beeinflusst.“ (Internetquelle 9) Im Duden wird Selbstoptimierung deutlich kritischer - als ,jemandes [übermäßige] freiwillige Anpassung an äußere Zwänge, gesellschaftliche Erwartungen oder Ideale u. Ä.“ (Internetquelle 10) definiert. Im Alltag gilt Selbstoptimierung als stetiger Versuch, Verhaltensweisen zu verbessern, um gesünder, bewusster und glücklicher zu leben. Es zählen jedoch auch gezielte Veränderungen des Aussehens etc. dazu, die das Individuum attraktiver, und ,schöner' machen sollen, wobei die Bestimmung von Attraktivität bzw. Schönheit je nach Kultur und gesellschaftlichen Idealen stark differieren kann.

Die Sozialwissenschaftler Oswald Balandis und Jürgen Straub beschreiben den sich unentwegt verbessernden Menschen des späten 20. und frühen 21. Jahrhunderts als interessantes und expandierendes Forschungsfeld. (vgl. Ballandis/Straub 2018) In den komplexen modernen Gesellschaften entwickeln sich immer neue Optimierungsbegehren. Selbstoptimierung wird damit zum Orientierungsmuster für die individuelle Lebensgestaltung. (vgl. Fenner 2020, S. 4). Auch die Optimierung des Selbst in Zeiten des Web 2.0 umfasst eine 
Vielzahl vermeintlicher Verbesserungen der eigenen Person, deren Ziel ein glücklicheres Leben ist. Verbunden ist dies häufig mit einem ,wenn-dann' Versprechen. Wenn ich mich gesünder ernähre, wenn ich mehr Sport treibe, wenn ich schöner aussehe, dann werde ich länger leben, attraktiver, ausgeglichener etc. und damit letztendlich auch glücklicher sein. Neben Fitness, Gesundheit und Schönheit können aber auch „Sexualität und Schlaf, alle möglichen physischen und kognitiven Leistungen, emotionale Fähigkeiten und soziale Kompetenzen“" (Bellandis/Straub 2018, S. 131) optimiert werden. Im Zentrum aller Bemühungen steht das Anstreben und Erreichen eines - bis auf weiteres und vergleichsweise - idealen Zustandes.

Selbstoptimierer`innen gelten dabei vielen als Prototyp des modernen Individuums, das versucht, aus seinem Leben das Maximum herauszuholen auf der Suche nach dem „tolleren Ich“ (Friedrichs 2013), wie das ZEIT-Magazin titelte. Die Ratgeberliteratur zur Selbstoptimierung ist breit gefächert und zeigt, dass dieses Thema auch jenseits von Instagram und YouTube außerordentlich populär ist. Bereits die Titel verweisen auf die Verantwortung des Einzelnen und die Verbindung von Gesundheit und Glück: „Better Body, better Brain. Das Handbuch zur Selbstoptimierung von Körper und Geist“" , „Glücksorgan Gehirn. Selbstoptimierung beginnt im Kopf “3 ${ }^{“ 3}$ oder „Erschaffe die beste Version von Dir. Dein Neustart in ein gesundes und glückliches Leben“4
„Der Selbstoptimierungstrend hat einen riesigen Selbstentwicklungs- markt mit einer Fülle an Lebenshilfe- und Ratgeberliteratur, Coa- ching-Angeboten, Blogs, Vorträgen, Seminaren und Workshops z. B. zum lebenslangen Lernen, Zeit- und Selbstmanagement, Willens- und Motivationstraining hervorgebracht" (Fenner 2020, S. 5).

In der deutschsprachigen Presse wird die Selbstoptimierung hingegen eher kritisch beobachtet. „Warum Selbstoptimierung in die Irre führt“ erklärt das Handelsblatt. Bereits eine kurze Recherche in der häufig tendenziösen Be-

2 Von Anja Leitz, 2016, München: Riva-Verlag.

3 Von Gabriele Rossbach, 2018, Wiesbaden: Springer Verlag.

4 Von Ralf Bohlmann, 2017, München: Riva Verlag. 
richterstattung führt zu ergänzenden Begriffen wie „Sucht“, „Selbstbetrug“, „Diktat“ oder „Hamsterrad“. „Selbstoptimierung mit Suchtpotential. Der ewige Kampf um den perfekten Body“ titelt der Tagesspiegel. Oder: „Wie geht man damit um, wenn ein Familienmitglied ohne Rücksicht auf Verluste, seinen Plan der eigenen Selbstoptimierung verfolgt?" fragt der Podcast Ein besseres Leben des Radiosenders Bayern 1. In vielen Artikeln und Beiträgen ist von sozialem Druck und unerfüllbaren Erwartungen die Rede, davon, dass Menschen sich freiwillig einem unbarmherzigen Leistungsideal unterwerfen und dabei ihr wahres Selbst verlieren.

\section{$4 \quad$ Das ideale Ich oder das mediale Ich? Selbstoptimierung im Netz}

Die Selbstoptimierung hat im digitalen Zeitalter die technische Vermessung des Selbst entdeckt, die vor allem mit der 2007 im Internet gestarteten Quantified Self-Bewegung in Verbindung gebracht wird. Die Bewegung versteht sich dabei als „eine Gemeinschaft von Anwendern und Anbietern von Self-Tracking Lösungen“ (vgl. Internetquelle 11). Neben dem Austausch über Erfahrungen mit den angewandten Mitteln und Methoden zur Erfassung von Daten aus allen Lebensbereichen, stehen die persönlichen Erkenntnisse, die aus den Daten abgeleitet und in Veränderungen umgesetzt werden, im Mittelpunkt. (ebd.) Neben gesundheitlich und körperlich orientierten Anwendungsbereichen findet der Grundgedanke der Quantified-Self-Bewegung seine Umsetzung auch in der Betrachtung von täglichen Routinen, die eingeschränkt oder verbesserte werden sollen. Alltägliche Handlungen wie Emailverkehr, die Smartphone-Nutzung oder der Schlafrhythmus können so in den Fokus der individuellen Analyse oder des gemeinschaftlichen Vergleichs rücken. Dazu dient die Veröffentlichung von Erfahrungsberichten und Präsentationen auf der Website quantifiedself.com, welche die amerikanischen Wired-Journalisten Gary Wolf und Kevin Kelly 2007 entwickelten. In den folgenden Jahren entstanden weitere Quantified-Self-Gruppen auf der ganzen Welt, 2012 gründete Florian Schumacher eine deutsche Sektion. Internationale Konferenzen mit Anwender*innen, Entwickler*innen, Journalist ${ }^{*}$ innen und Unternehmens- 
vertreterinnen aus der Gesundheitsbranche finden jährlich unter großem Zuspruch statt.

Die Vermessung des Selbst und das zeitgenössische Optimierungsbegehren ist ein Trend, der bereits seit einigen Jahren wissenschaftlich beobachtet wird. So zeigt die TA-Swiss-Studie, dass der Drang, neue Erkenntnisse über sich selbst zu gewinnen, durch die Nutzung von Geräten und Applikationen zur Selbstvermessung und Selbstoptimierung stark zugenommen hat. (vgl. Meidert u.a. 2018) Die Daten aus Fitnessarmbändern, eingebauten Sensoren in Kleidungsstücken oder aus einer der über 100.000 Gesundheits-Apps dienen dabei als Grundlagen für Entscheidungen und Handlungen, als Ansporn sowie zur Dokumentation und zum Austausch mit Gleichgesinnten.

Die Zahl der Apps zur Optimierung im Bereich Fitness, Wellness und Gesundheit wächst stetig und immer neue Angebote kommen auf den Markt. An dieser Stelle soll die Bandbreite der Angebote nur oberflächlich anhand der beiden Angebote Argus - Your Fitness Tracker und Sleep Circle Alarm Clock skizziert werden. Die von Azumio Inc. entwickelte käufliche App Argus - Your Fitness Tracker bietet ihren Benutzer*innen eine Rundumkontrolle in Sachen gesunde Lebensweise. Mit Argus kann protokolliert werden, was man isst, wie oft man trinkt, wie viele Kalorien man verbrennt, welche Wegstrecken man zurücklegt und wie es mit dem Herzrhythmus aussieht. Zur Verbesserung seiner Daten legt man bestimmte Ziele fest, zum Beispiel, wie viele Gläser Wasser man pro Tag trinken möchte. Tabellen listen dann die Erfolge oder Misserfolge des Tages auf. Die kostenlose App Sleep Cycle Alarm Clock verspricht hingegen, den richtigen Moment zum Aufwachen zu finden. Mithilfe von Ton- und Vibrationsanalyse trackt die Anwendung Bewegungen im Schlaf und sucht dann innerhalb eines 30 Minuten langen Zeitfensters vor der Weckzeit den optimalen Zeitpunkt für den Alarm. Dadurch fühlt man sich nach dem Aufwachen ausgeruht und energiegeladen, so die Entwickler (Internetquelle 12). 


\section{Antworten zum Schluss: Was macht nun wirklich glücklich?}

Die Popularität der Selbstvermessung mit ihren Versprechungen für ein gesünderes und glücklicheres Leben ist groß und wird durch immer neue Produktideen befeuert. Allerdings zeigen Forschungen auch, dass ein Großteil der heruntergeladenen Gesundheits-Apps und Fitnesstracker nach kurzer Zeit nicht mehr verwendet werden. (vgl. Meidert u.a. 2018) Die TA-Swiss-Studie sieht vor allem zwei problematische Entwicklungen: Zum einen werden viele Produkte von Hersteller*innen aus dem technischen Bereich entwickelt und produziert. „Gesundheitsapps fehlt es deshalb oft an evidenzbasierten Inhalten, d.h. diese entsprechen nicht dem aktuellen medizinischen-therapeutischen Wissensstand.“ (vgl. Internetquelle 13) Zum anderen besteht die Gefahr, dass Selbstvermessung den falschen Eindruck erweckt, jedes Individuum können „seinen Gesundheitszustand vollständig selbst bestimmen und kontrollieren“ (ebd.). Und könne somit auch sein Glück maßgeblich beeinflussen.

Dabei zeigt die Glücksforschung in vielen Studien, dass Glück weniger im Kreisen um sich selbst als in der Interaktion mit anderen Menschen zu finden ist. Bereits 2008 kam eine Studie der University of British Columbia und der Harvard Business School zum dem Schuss: „Can money buy you happiness? Yes - so long as you spend the money on someone else. According to new research, giving other people even as little as $\$ 5$ can lead to increased well-being for the giver." (Internetquelle 14) Für wen oder was genau man das Geld gibt, spiele dabei keine Rolle. Die Quintessenz bleibt: Wer gerne etwas für Andere gibt, wird letzten Endes mit einem glücklicheren Leben belohnt. Und die Langzeitstudie von Wissenschaftler*innen der Harvard University (Internetquelle 15) zeigt über 75 Jahre hinweg: Es gibt nur eine Sache, die zu einem wirklich langanhaltenden glücklichen Leben führt: Gute Beziehungen! Gemeint sind damit tiefe Bindungen mit anderen Menschen, sei es mit Ehepartner*innen oder aber Freund ${ }^{\star}$ innen und Familienmitgliedern. Somit müsste der Titel dieses Beitrages für die Suche nach dem Glück eigentlich nicht das „ideale Ich“ thematisieren, sondern das Streben nach einem gemeinsamen „Wir“. 


\section{Literatur}

Ballandis, Oswald/Jürgen Straub (2018): Selbstoptimierung und Enhancement. Der sich verbessernde Mensch - ein expandierendes Forschungsfeld. In: Journal für Psychologie, 26(1), S. 131-155.

Dallmer, Jochen (2020): Glück und Nachhaltigkeit. Subjektives Wohlbefinden als Leitmotiv für nachhaltige Entwicklung. Bielefeld: transcript Verlag.

Fenner, Dagmar (2020): Selbstoptimierung. http://www.bpb.de/gesellschaft/umwelt/ bioethik/311818/selbstoptimierung

Friedrichs, Julia (2013): Selbstoptimierung. Das tollere Ich. In: ZEIT-Magazin Nr. 33

Helliwell, John/Layard, Richard/Sachs, Jeffrey (2019). World Happiness Report 2019. New York: Sustainable Development Solutions Network.

Meidert, Ursula u.a. (Hg.) (2018): Quantified Self - Schnittstelle zwischen Lifestyle und Medizin. Zürich: vdf Hochschulverlag.

Meißner, Stefan (2017): Ungeplante Selbstverhältnisse. Quantified Self als Phänomen jenseits von Steuerung und Planung. In: Koch, Matthias/Köhler, Christian/Othmer, Julius (Hg.): Planlos! Zu den Grenzen von Planbarkeit. München: Wilhelm Fink Verlag (Schriftenreihe des Graduiertenkollegs „Automatismen“), S. 137-150.

\section{Internetquellen}

Internetquelle 1: https://www.thehappinessmuseum.com/about (letzter Aufruf 25.08.2020)

Internetquelle 2: https:/www.un.org/Depts/german/gv-66/band3/ar66281.pdf Internetquelle 3:https://www.un.org/Depts/german/gv-65/band3/ar65309.pdf Internetquelle 4: https://worldhappiness.report/

Internetquelle 5: https://www.sz.de/dpa.urn-newsml-dpa-com-20090101190320-99-471320

Internetquelle 6: https://www.dpdhl.com/de/presse/specials/gluecksatlas.html\# onetrust (letzter Aufruf 10.08.2020)

Internetquelle 7 : https://www.sinus-institut.de/veroeffentlichungen/meldungen/ detail/news/gluecks-studie-deutsche-sind-gluecklich-und-optimistisch/news-a/ show/news-c/NewsItem/ (letzter Aufruf 25.08.2020)

Internetquelle 8: https://www.ovgu.de/Universit\%C3\%A4t/Im+Portrait/ Profilierungsschwerpunkte/Forschung+_+Transfer/Auf+der+Suche+nach+ dem+Gl\%C3\%BCck.html (letzter Aufruf 10.08.2020)

Internetquelle 9: https://www.duden.de/rechtschreibung/Selbstoptimierung

Internetquelle 10: https://www.spektrum.de/lexikon/neurowissenschaft/ selbstoptimierung/11639

Internetquelle 11: http://qsdeutschland.de/info/ (letzter Aufruf 10.08.2020) 
Internetquelle 12: https://www.sleepcycle.com/ (letzter Aufruf 10.08.2020)

Internetquelle 13: https:/www.zhaw.ch/de/gesundheit/forschung/ergotherapie/ projekte/quantified-self/

Internetquelle 14: https://hbswk.hbs.edu/item/spending-on-happiness

Internetquelle 15: https://www.adultdevelopmentstudy.org/grantandglueckstudy 

Alexandra Ebel, Halle (Saale)/Rudolf Hientz, Frankfurt am Main/Ursula Hirschfeld, Halle (SaAle)/

Robert Skoczek, Halle (SaAle)

\section{Zur Transkription von Standardsprache in Aussprachedatenbanken: Konzepte, Probleme, Lösungen}

Zusammenfassung: Ausgehend von der aktuellen Terminologiediskussion zum Begriff "Aussprachestandard“ im deutschsprachigen Raum werden Konzepte, Probleme und Lösungen für die Transkription in Aussprachenachschlagewerken angesprochen und diskutiert, wobei speziell die phonetische Transkription in Aussprachedatenbanken im Fokus steht.

Stichworte: Standardaussprache, Aussprachedatenbank, Normphonetik, Transkription, Deutsch als Fremdsprache

Abstract: Based on the current discussion of the term "Aussprachestandard" (standardized pronunciation) in the German speaking area, concepts, problems and solutions for the transcription in pronunciation dictionaries will be mentioned and discussed. A special focus is placed on phonetic transcription in pronunciation databases on the internet.

Keywords: standard pronunciation, pronunciation database, phonetic norm, transcription, German as a foreign language

Ausgehend von der aktuellen Terminologiediskussion zum „Aussprachestandard" wird derzeit die Transkription in Aussprachenachschlagewerken diskutiert, und zwar speziell auch für die Transkription in Aussprachedatenbanken. Im Rahmen eines Kolloquiums zum Thema „Zur Transkription von Standardsprache in Aussprachedatenbanken: Konzepte, Probleme, Lösungen“, das im 
Vorfeld der Kleine Fächer-Wochen stattfand, wurden zu den Teilthemen dieses Beitrags von den Autorinnen und Autoren Vorträge gehalten, die im Folgenden zusammengefasst werden.

\section{Zur Darstellung der Standardaussprache in Aussprachenachschlagewerken (Ursula Hirschfeld)}

\subsection{Aussprachenachschlagewerke}

Aktuell stehen als spezielle Nachschlagewerke für die Aussprache des Deutschen - neben zahlreichen anderen ein-, zwei- oder mehrsprachigen Nachschlagewerken mit Hinweisen zur Aussprache - drei Aussprachewörterbücher und zwei Aussprachedatenbanken zur Verfügung:

a. Deutsches Aussprachewörterbuch (DAWB, 2009, Krech et al.), dazu wird zur Zeit eine Datenbank entwickelt (vgl. unter 2.3),

b. Duden - Das Aussprachewörterbuch (Duden-AWB, 2015, 7. Auflage, Kleiner/Knöbl - Mangold wird im Impressum als Mitautor angegeben, nicht aber auf der Titelseite),

c. Österreichisches Aussprachewörterbuch und österreichische Aussprachedatenbank (2007, Muhr),

d. ARD-Aussprachedatenbank (Internetquelle 1), sie wird im 3. Kapitel dieses Beitrags von Rudolf Hientz vorgestellt.

Diese Nachschlagewerke unterscheiden sich in der Terminologie, in der Definition von Standardaussprache, in der Einbeziehung regionaler Formen, in den Transkriptionsregelungen und in ihren Positionen zur Eindeutschung fremder Namen und Wörter. Im Folgenden wird auf die ersten drei Aspekte eingegangen. 


\subsection{Terminologie und Definitionen}

Seit Beginn der Orthoepieforschung im 19. Jahrhundert gelten Überregionalität, Deutlichkeit, Verständlichkeit, Einheitlichkeit und Schriftnähe als Kriterien für eine Sprechweise, die man heute als Standardaussprache bezeichnet. In den aktuellen Nachschlagewerken wird Standardaussprache unter (teilweiser) Einbeziehung dieser Kriterien unterschiedlich definiert, wobei sich besonders das Kriterium der Überregionalität als instabil erweist.

Das DAWB (2009) verwendet den Begriff Standardaussprache, ebenso wie die Vorgängerpublikationen "Großes Wörterbuch der deutschen Aussprache" (Krech et al. 1982) und "Wörterbuch der deutschen Aussprache" (Krech et al. 1964), letzteres in der Formulierung „allgemeine deutsche Hochlautung als Standardaussprache“. Die Standardaussprache (vgl. Krech et al. 2009, 7)

- ist dialektneutral und enthält keine regional gefärbten umgangssprachlichen Formen,

- wird überregional und in allen sozialen Gruppen verstanden,

- wird besonders in offiziellen öffentlichen Situationen genutzt bzw. erwartet, ihre Verwendung ist in solchen Situationen prestigefördernd,

- ist durch unterschiedliche Grade der Artikulationspräzision (phonostilistische Differenzierungen) gekennzeichnet,

- ist kodifiziert und kann als explizite Norm regulative Funktionen erfüllen.

Das Duden-AWB (2015) bezeichnet die „bundesdeutsche überregionale Standardaussprache“ als „Gebrauchsstandard“. Im Vorwort (S. 6) wird von einer „authentischen Gebrauchsnorm“ der deutschen Aussprache gesprochen. Die hier definierte Standardaussprache (vgl. S. 31f.)

- ist eine Gebrauchsnorm, die der Sprechwirklichkeit nahekommt, weil sie neben dem Sprachgebrauch von überregional tätigen Berufssprecherinnen auch die in der Bevölkerung übliche Standardaussprache berücksichtigt, 
- enthält in der Mehrzahl überregional gebräuchliche Ausspracheformen, aber auch solche, die innerhalb des deutschen Sprachraums nur national oder großregional üblich sind,

- wird phonologisch grundsätzlich einheitlich dargestellt, das phonologische Muster bildet dabei die überregionale Standardaussprache, wie sie in Deutschland typischerweise von Berufssprecher*innen in den Medien verwendet wird,

- ist zwar in ihren Grundzügen schriftnah, folgt aber im Zweifelsfall auch dem tatsächlichen Sprachgebrauch, wenn dieser Diskrepanzen zu regelhaften Schreibungs-Aussprache-Korrespondenzen aufweist.

Muhr (2007) geht davon aus, dass es (in Österreich) nicht nur einen Aussprachestandard gibt, sondern „viele verschiedene, die überregionalen, regionalen, öffentlichen, privaten oder situativen Charakter haben“" (S. 30). Er beschreibt neben der österreichischen auch die bundesdeutsche und deutschschweizerische Standardaussprache als „Standardformen“. Für die österreichische Varietät wird von einer „Medienpräsentationsnorm“ (S. 7), einer „Modellaussprache" (S. 29) ausgegangen. Die österreichische Standardaussprache als mediale Präsentationsnorm (vgl. S. 28) ist

- überregional verständlich/gebräuchlich,

- innerhalb „definierter politischer Einheiten“ akzeptiert,

- sozial anerkannt,

- einer identitätsstiftenden Norm angehörig.

\subsection{Einbeziehung regionaler Formen in Aussprachewörterbüchern}

Die Abgrenzung zwischen Standardsprache und Regionalsprachen wird im Metzler Lexikon Sprache so formuliert:

„Innerhalb eines heterogenen und dynam. Gesamtsprachsystems wird die Standardsprache von der Regionalsprache unterschieden. Unter Standardsprache wird eine Varietät verstanden, die frei ist von salien- 
ten (d. h. für die Sprachteilhaber auffälligen) Regionalismen." (Glück/ Rödel 2016, 145).

Damit wird die Standardsprache gleichzeitig als frei von Regionalismen definiert. Dies gilt auch für die Aussprache als Teilbereich der Standardsprache. In Aussprachenachschlagewerken, die die Standardaussprache normativ darstellen, also einheitliche „Muster“ zur Orientierung vorgeben, sollte deshalb am Aspekt der Überregionalität festgehalten werden. Für die deskriptive Dokumentation anderer Ausspracheformen gibt es andere Publikationsmöglichkeiten: Variantenwörterbücher, Dialektwörterbücher und -datenbanken, Datenbanken für gesprochenes Deutsch, für Alltagssprache.

Für die Nutzer*innen von Aussprachenachschlagewerken sollte ganz klar erkennbar sein, was sie beinhalten und ob sie deskriptiv oder normierend angelegt sind. Maria Bonner stellt in diesem Zusammenhang die Frage, ob die aktuelle Auflage des Duden-Aussprachewörterbuchs für Deutschlernende eine hilfreiche Orientierung ist, und stellt fest:

„Ein deskriptiv dokumentierendes Aussprachewörterbuch - neben einem normierenden - wäre durchaus ein Desiderat für das Deutsche, es in einer Neubearbeitung des Duden-Aussprachewörterbuchs quasi nebenbei zu versuchen, ist wenig erfolgversprechend und für Nutzer vermutlich auch wenig befriedigend. Lerner wollen wissen, welche Aussprache als vorbildlich gelten soll, für sie ist Variation eher verwirrend, und Muttersprachler benutzen gerade ein Werk mit dem Namen Duden mit dem Anspruch einer Richtschnur." (Bonner $(2017,4)$. 
Zur Vokalquantität wird im Duden-Aussprachewörterbuch z. B. angegeben:

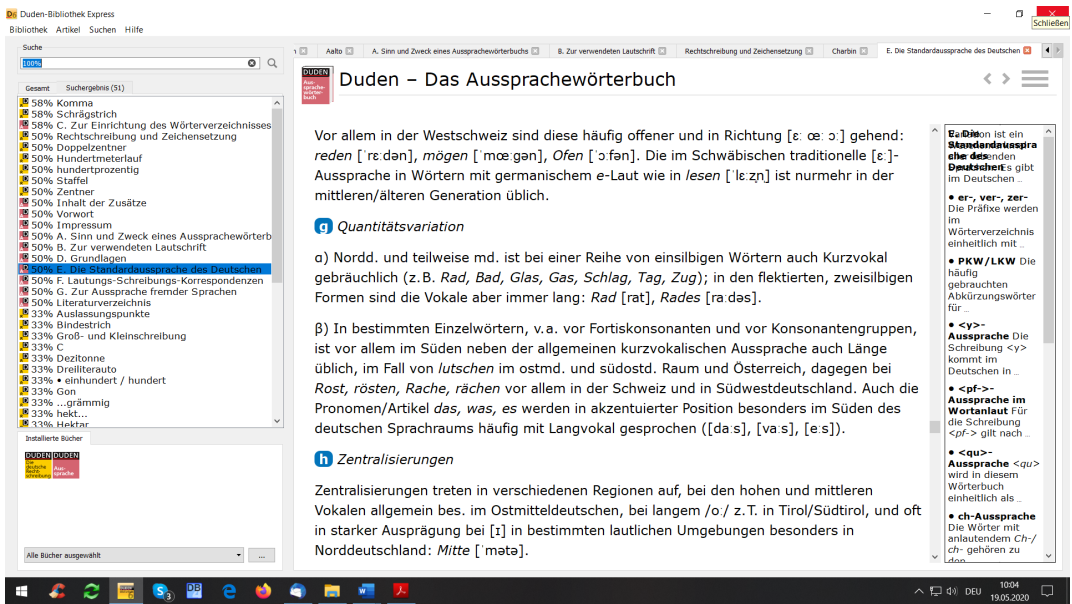

Abb. 1: Quantitätsvariation im Duden-Aussprachewörterbuch (Kleiner/Knöbl 2015, 56; Abb. entnommen aus der elektronischen Fassung)

Auch andere Nutzer*innen kann die Beschreibung von Varianten in einem Aussprachewörterbuch irritieren. Lehrende im Bereich Deutsch als Fremdsprache, Sprecher*innen in den elektronischen Medien, Schauspieler*innen, Rezitatorinnen, Sängerinnen und Gesangspädagog^innen, sprachheiltherapeutisch und -pädagogisch Tätige, diese Berufssprecher/-sänger`innen auf phonetischem, rhetorischem und (sprech)künstlerischem Gebiet Auszubildende, Persönlichkeiten, die in Wissenschaft, Kultur, Wirtschaft und Politik in der Öffentlichkeit wirken sowie Pädagog*innen aller Fachrichtungen und in allen Institutionen (vgl. Krech et al. 2009, 6 f.) brauchen einheitliche und klare Angaben für eine überregionale, allgemein verständliche und akzeptierte Aussprache. 


\section{Möglichkeiten und Grenzen der Transkription von Standardsprache in Aussprachedatenbanken (Alexandra Ebel)}

\subsection{Aussprachewörterbücher}

Aktuell gibt es im deutschsprachigen Raum drei Aussprachewörterbücher und zwei Aussprachedatenbanken (vgl. Kap. 1.1).

Neben theoretischen Hinweisen zur Standardaussprache, die in die Nutzung eines Aussprachewörterbuchs einführen, bildet das Wörterverzeichnis den Hauptteil. Darin sind die einzelnen Stichwörter linear angeordnet und alphabetisch sortiert (vgl. Abb. 2).

429

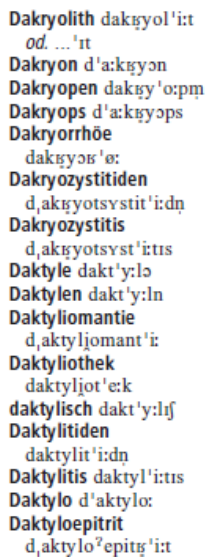

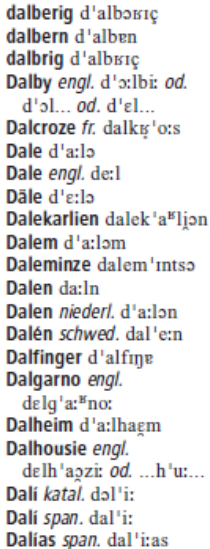

Dalriada da:lsị 'a:da: Dalrymple engl. dels' 'Impl od. d'el. Dalsgaard dän. d'alsğa: ${ }^{\mathrm{K}}$ Dalsgård dản. d'alsģa: ${ }^{\mathrm{K}} \mathrm{t}$ Dalski serb./kroat. d3'alski: Dalsland d'a:Islant Dalton engl. d's:ltn Daltonismus

dalton'ısmus

Daluege dal'y:gə

Dalwigk d'a:lvik

Daly engl. d'e:li:

Dam dän. dam

Dam niederl. dam

Dama d'a:ma:

Damage engl. d'emots

damalig d'a:ma:lıç

damals d'a:ma:ls

Damanhur damanh' $u:^{\mathrm{E}}$

Damão port. dam'ax

Damara dam'a:sa: od. d'a:...
Damoklesschwert
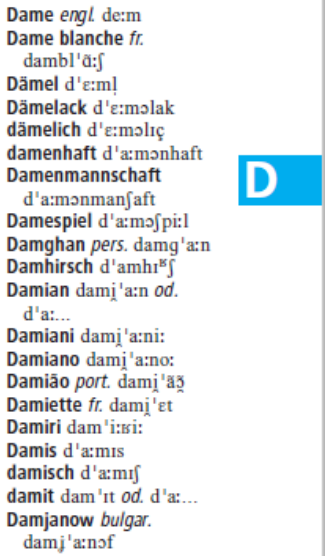

Abb. 2: Auszug aus dem Deutschen Aussprachewörterbuch (Krech et al. 2009)

Die normphonetische Transkription der Aussprache erfolgt mittels Internationalem Phonetischem Alphabet (IPA). Eine weitere Lautschrift ist aus Platzgründen nicht möglich und würde vermutlich auch die Übersichtlichkeit vermindern. Auch Zusatzinformationen können nur sehr eingeschränkt gegeben werden. Im DAWB beschränkt man sich beispielsweise auf die Angabe 
der Herkunft - jedoch nur, wenn die Aussprache nicht den deutschen Phonem-Graphem-Beziehungen entspricht - und auf Präzisierungen, wenn ein gleich geschriebener Eintrag mehrere Bedeutungen hat, wie August (Monat) [asg'ust] gegenüber August (Name) ['asgost] (Krech et al. 2009, 340). Des Weiteren muss die Darstellung von Aussprachevarianten aus Platzgründen verkürzt erfolgen (vgl. Abb. 2, Eintrag Dakryolith). Hierbei wird lediglich die relevante Silbe transkribiert, der Rest ist aus der vollständig angegebenen Variante zu schließen. In den meisten Fällen ist dies sicherlich problemlos möglich, doch es gibt auch Zweifelsfälle. In Abb. 2 ist zum Beispiel beim Eintrag Damian davon auszugehen, dass auch in der Variante mit Erstsilbenakzentuierung das [a:] in der letzten Silbe lang gesprochen werden muss. Denkbar wäre aber auch eine Realisierung als [d'a:mian].

Die Form eines Wörterbuches lässt also neben der Angabe der Aussprache nur wenige zusätzliche Informationen zu. Außerdem bildet es lediglich einen bestimmten Stand ab, sowohl im Hinblick auf die enthaltenen Stichworteinträge als auch auf die normphonetischen Empfehlungen. Für Änderungen und Ergänzungen wird eine neue Auflage nötig. Zuletzt sei angemerkt, dass Nutzende die korrekte Schreibung des gesuchten Lemmas kennen müssen, um dieses im Wörterverzeichnis zu finden. Insbesondere wenn von einem falschen Anfangsbuchstaben ausgegangen wird, ist zu erwarten, dass der Eintrag nicht gefunden wird.

Wie sich gezeigt hat, bestehen bei Aussprachekodizes in Buchform mehrere Einschränkungen für Herausgebende und Nutzende. Durch die rasante Entwicklung des World Wide Webs und seiner Möglichkeiten ergeben sich jedoch Chancen, die Einträge neu aufbereitet abzubilden.

\subsection{Aussprachedatenbanken}

Unter Aussprachedatenbanken sollen hier Datenbankanwendungen im Internet verstanden werden, über die Nutzende bestimmte Informationen bzw. Daten - in diesem Fall bzgl. der Aussprache - aus einer im Hintergrund befindlichen Datenbank abrufen können. In welcher Form diese Informationen aufbereitet werden und welche Daten überhaupt zur Verfügung gestellt werden, ist bei den bestehenden Aussprachedatenbanken verschieden. 
Die Möglichkeiten, die sich durch Aussprachedatenbanken bieten, sind jedoch sehr vielfältig und mit Sicherheit auch noch nicht vollständig ausgelotet. Einige Vorteile der Datenbanken - auch im Vergleich zu Aussprachewörterbüchern - sollen an dieser Stelle genannt werden:

- Im Gegensatz zum linear aufgebauten Wörterbuch können Webseiten und somit auch die Datenbankanwendungen hypertextuell aufbereitet und genutzt werden. Das bedeutet, dass Verlinkungen zwischen einzelnen Einträgen sowie zu anderen Webseiten möglich sind. Dadurch ist es einerseits denkbar, dass Nutzende von einem Eintrag aus direkt zu den damit verbundenen Ausspracheregeln geleitet werden könnten. Andererseits müssen Zusatzinformationen wie etymologische Angaben nicht in der Datenbank vorhanden sein, sondern man kann auf bereits im Internet bestehende Angebote verweisen.

- Die Einträge der Datenbank können multimedial aufbereitet werden, so dass zusätzlich zur textuellen Darstellung auch Audio- oder sogar Videoaufnahmen zur Verfügung gestellt werden können. Insbesondere die Unterstützung durch Tonaufnahmen stellt für viele Nutzende eine Erleichterung dar, weil sie sich im Umgang mit Lautschriften nicht sicher genug fühlen. Videoaufnahmen könnten sich z. B. für spezielle Nutzer*innengruppen anbieten, wie Lernende von Deutsch als Fremdsprache, für die auch die Mundmotorik bei der Artikulation eines Wortes interessant ist.

- Aussprachedatenbanken bieten verschiedene Suchfunktionen, welche die Nutzung deutlich vereinfachen können. So ist es denkbar, dass bereits Einträge vorgeschlagen werden, auch wenn das gesuchte Lemma noch nicht vollständig ins Suchfeld eingegeben wurde. Dadurch können Nutzende Zeit sparen und Unsicherheiten bzgl. der korrekten Schreibung können abgefangen werden. Den gleichen Effekt hat eine unscharfe Suche, wie sie auch durch die Suchmaschine Google bekannt ist: Ein falsch geschriebenes Wort wird trotzdem gefunden bzw. es werden Vorschläge gemacht, welches Wort gemeint sein könnte. Hinzu kommen Möglichkeiten für Suchen nach Buchstabenkombinationen, auch positionell, oder nach bestimmten Silben. Und na- 
türlich ist auch eine Suche auf Lautschriftebene realisierbar, sodass ebenfalls nach Lautkombinationen gesucht werden kann.

- Neben den Suchfunktionen sind auch verschiedenste Filtermöglichkeiten denkbar, welche natürlich von den Informationen abhängen, die in der Datenbank hinterlegt sind. Nutzende könnten sich somit Einträge einer ausgewählten Herkunftssprache anzeigen lassen oder nur Lemmata mit einer bestimmten Silbenzahl. Auch eine Kombination verschiedener Filter stellt in modernen Datenbankanwendungen kein Problem dar.

- Generell können zu den Einträgen beliebig viele Zusatzinformationen geliefert werden, ohne dass dadurch die Übersichtlichkeit beeinträchtigt sein muss. Zusätzlich zum IPA könnte eine weitere Art der Lautschrift (bspw. SAMPA) angegeben werden. Verschiedene Aussprachevarianten müssten nicht verkürzt dargestellt werden und bei fremdsprachigen Einträgen könnten ausführlichere Angaben zu Herkunfts- und ggf. Mittlersprachen sowie zum Eindeutschungsgrad gemacht werden.

Derzeit (Stand Sommer 2020) lässt sich über das Internet auf drei Aussprachedatenbanken zugreifen:

ARD-Aussprachedatenbank. Diese ist nur für berechtigte Personen, d. h. vor allem Journalist*innen zugänglich. Sie wurde 1997 gegründet und seitdem stetig erweitert. Hauptsächlich sind in der ARD-Aussprachedatenbank Personen- und Ortsnamen enthalten, da diese für Rundfunksprechende die größte Herausforderung darstellen. Eine detaillierte Beschreibung der Arbeitsweise der Datenbank ist in Kapitel 3 dieses Beitrags zu finden.

Forvo. Das Aussprachewörterbuch. Interessanterweise nennt sich diese Datenbank selbst „Aussprachewörterbuch“, obwohl ihr kein gedrucktes Wörterbuch zugrunde liegt. Forvo wurde 2008 gegründet, „mit der Mission, die mündliche Kommunikation über die Kulturen hinweg zu befördern“ (Internetquelle 2). Erfüllt werden soll dieses Ziel, indem Muttersprachler*innen aus verschiedenen Sprachen selbst eingesprochene Audioaufnahmen eines 
bestimmten Lemmas hochladen. Eine Transkription wird nicht dazu geliefert. Da auch mehrere Aufnahmen verschiedener Personen pro Lemma möglich sind, kann es vorkommen, dass für einen Eintrag unterschiedliche Aussprachevarianten in der Datenbank abrufbar sind. Dadurch wird zwar Varianz, z. B. auf dialektaler Ebene, abgebildet, aber die Nutzenden werden mit der Entscheidung, welche Variante sie bevorzugen sollten, allein gelassen. Zwar gibt es eine Bewertungsfunktion unter jeder Audiodatei, mit der diese als gut oder schlecht eingeschätzt werden kann, doch es sind längst nicht alle Einträge mit Bewertungen versehen und vor allem ist nicht ersichtlich, nach welchen Kriterien die einzelnen Nutzenden ihre Einschätzung vorgenommen haben (z. B. Stimmklang, Artikulationspräzision, technische Qualität der Aufnahme).

adaba: Österreichische Aussprachedatenbank. Diese Datenbank ist in Verbindung mit dem Österreichischen Aussprachewörterbuch unter der Gesamtleitung von Rudolf Muhr entstanden. Das Projekt hat zum Ziel, die österreichische Standardvarietät zu dokumentieren und kontrastiv zur bundes- und zur schweizerdeutschen Varietät abzubilden (vgl. Internetquelle 3). Die Datenbank enthält phonetische Transkriptionen und Audioaufnahmen von ca. 1/3 der Lemmata sowie von 52 Mustertexten.

\subsection{Die Deutsche Aussprachedatenbank (DAD)}

An der Abteilung Sprechwissenschaft und Phonetik der Martin-Luther-Universität Halle-Wittenberg wird seit einiger Zeit an einer weiteren Aussprachedatenbank gearbeitet, die als Weiterentwicklung des Deutschen Aussprachewörterbuchs den darin enthaltenen Wortschatz ins Internet bringen soll.

Nach derzeitigem Stand sind alle ca. 130.000 Stichwörter in die Datenbank eingepflegt und mit Zusatzinformationen zu Silbenzahl, Akzentmuster und neben IPA- auch SAMPA-Transkription versehen (vgl. Abb. 3). Die Suche und Filterung nach diesen Informationen ist ebenso möglich, wie die gezielte Suche nach Lautkombinationen. 
- Hauptseite

- Erweiterte Suche im

Aussprachewörterbuch

- Über die DAD

- Hinweise zur Eindeutschung

- Hinweise zur Transkription

- Nutzungsbedingungen

- Redaktionsportal

- Impressum

\section{Dakryozystitiden}

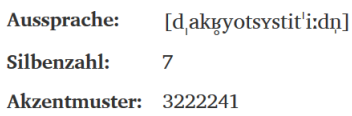

SAMPA: $\quad$ d\%ak.R0y.o.tsYs.ti.t"i:.dn =

Abb. 3: Darstellung eines Stichworts in der Deutschen Aussprachedatenbank (DAD)

Weiterführende Pläne sehen vor, die Einträge mit Audiodateien zu hinterlegen, Verlinkungen einzufügen und den Grundwortschatz für Deutsch als Fremdsprache hervorzuheben. Außerdem soll durch Überarbeitungen im Back- und Frontend die Benutzungsfreundlichkeit erhöht werden. Längerfristig ist es zudem denkbar, Lemmata nach Themen geordnet anzubieten (z. B. Hauptstädte, Gewässer, Lebensmittel), das Angebot an Hörbeispielen zu vergrößern (verschiedene Sprecherinnen, komplette Sätze) oder mehr Aussprachevarianten aufzunehmen.

\section{Anforderungen an Ausspracheempfehlungen bei öffentlich- rechtlichen Rundfunkanstalten (Rudolf Hientz)}

\subsection{Arbeitsweise ARD-Aussprachedatenbank}

Radio- und Fernsehjournalist*innen werden in ihrer täglichen Arbeit mit einer Vielzahl fremdsprachiger Namen und Begriffe konfrontiert, sei es in der politischen Berichterstattung, im Sportbereich, bei der Präsentation von Kultur- und Musikprogrammen oder der Vorproduktion von Features, Dokumentationen oder Hörspielen. Sie brauchen daher eine kompetente Aufbereitung von Ausspracheempfehlungen mit einer schnellen Zugriffsmöglichkeit und einfachen Darstellung. 
Um diesen Anforderungen nachzukommen wurde die beim Hessischen Rundfunk betriebene Aussprachedatenbank (im Folgenden: ADB) 1997 als ARD-Gemeinschaftseinrichtung etabliert. Zugriff auf die ADB haben über ihre jeweiligen Intranets die neun Landesrundfunkanstalten der ARD, das Deutschlandradio, die Deutsche Welle, der ORF (Radio und Fernsehen), das Schweizer Radio und Fernsehen SRF, Rai Südtirol, das ZDF und das luxemburgische öffentlich-rechtliche Radio 100,7 Luxemburg. Daneben besteht für Mitarbeiter dieser Rundfunkanstalten die Möglichkeit, sich einen Zugang für mobile Endgeräte freischalten zu lassen. Dieser Zugang kann zu wissenschaftlichen Zwecken auch externen Nutzer*innen gewährt werden.

Der Arbeitsaufwand wird somit an einer Stelle gebündelt und die Mehrfacharbeit in den einzelnen Häusern entfällt. Die ADB-Redaktion besteht aus einem festangestellten Leiter und einem wechselnden Team von freien Mitarbeiter*innen. Die Redaktion arbeitet sowohl präventiv als auch abfrageorientiert. Sie sichtet täglich Agenturmeldungen und Nachrichten-Portale und recherchiert relevante Namen und Begriffe aus dem aktuellen politischen, sportlichen und kulturellen Tagesgeschehen. Großereignisse, wie z. B. Fußball-Weltmeisterschaften, Filmfestivals oder Buchmessen, werden mit einigen Wochen Vorlauf vorbereitet. Daneben besteht für die Zugriffsberechtigten die Möglichkeit, per E-Mail oder telefonisch Anfragen an die Redaktion zu stellen. Die Bandbreite der Anfragen bewegt sich hierbei von einem bestimmten Namen oder Begriff bis hin zu kompletten Manuskripten, die von den Mitarbeiter*innen gesichtet werden.

\subsection{Gemäßigte Eindeutschung}

Einträge in der $\mathrm{ADB}$ werden in einer gemäßigten Eindeutschung vorgenommen, die dem Leitsatz "So original wie möglich, so deutsch wie nötig“ folgt. Dabei sollen möglichst viele Merkmale der originalen Lautung erhalten bleiben, ohne dass es dabei zu größeren Kollisionen mit dem deutschen Lautsystem kommt. Als Basis dafür dienen Angleichungsregeln, die gemeinsam mit Muttersprachler*innen erarbeitet wurden. Der Grad der jeweiligen Eindeutschung ist dabei je nach Sprache unterschiedlich. Geläufige, etwa aus dem Schulunterricht bekannte Sprachen wie Englisch oder Französisch wer- 
den kaum eingedeutscht, weniger präsente Sprachen erhalten einen höheren Grad der Eindeutschung. Die Originallautung wird in der ADB in Klammern jeweils mit angegeben. Exemplarisch seien die folgenden drei Fälle aus dem Englischen, Spanischen und Finnischen aufgeführt:

\begin{tabular}{|c|c|c|}
\hline Lemma & Originalaussprache & Eindeutschung ADB \\
\hline Athens & 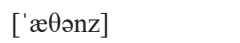 & 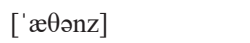 \\
\hline Valencia & [ba'len $\theta i a]$ & [va'lensia] \\
\hline Ähtäri & ['æhtæri] & ['Eçteri] \\
\hline
\end{tabular}

Im Fall der amerikanischen Stadt Athens findet keine Eindeutschung statt. Der ungerundete, fast offene Vorderzungenvokal [æ] bleibt ebenso erhalten wie der stimmlose dentale Frikativ $[\theta]$, eine Auslautverhärtung von $[\mathrm{z}] \mathrm{zu}[\mathrm{s}]$ findet nicht statt. Denkbar für eine stärkere Eindeutschung wäre eine Substitution von $[æ]$ mit dem ungerundeten, halboffenen Vorderzungenvokal $[\varepsilon]$, sowie das Markieren der Auslautverhärtung zu *[' $\varepsilon \theta ə n s]$. Eine noch stärkere Eindeutschung durch eine mögliche Substitution von $[\theta]$ durch den stimmlosen alveolaren Plosiv [ $\mathrm{t}$ ] oder Frikativ [s] würde von dem überwiegenden Teil der Zuhörer*innen/Zuschauer*innen wohl als befremdlich empfunden werden.

Bei der Eindeutschung von Valencia spielt zunächst die Orthografie eine Rolle. Der stimmhafte bilabiale Plosiv [b] befindet sich natürlich im deutschen Lautinventar und kann von einer*einem Deutsch-Muttersprachler*in problemlos ausgesprochen werden, ist aber als Aussprache des Graphems $\langle\mathrm{V}\rangle$ im Deutschen nicht verzeichnet. Um eine Kongruenz zwischen geschriebener und gesprochener Sprache herzustellen wird bei der Eindeutschung der stimmhafte labiodentale Frikativ [v] verwendet. Der ungerundete halbgeschlossene Vorderzungenvokal $[\mathrm{e}]$ in der betonten Silbe ist im Spanischen kurz. Im Deutschen ist der Kurzvokal [e] in betonter Stellung nicht möglich, weswegen bei der Eindeutschung entweder eine Längung [e:] oder eine Substitution mit $[\varepsilon]$ denkbar wäre. Aufgrund des nachfolgenden Konsonantenclusters, das in den meisten Fällen eine Kürzung des vorangegangenen Vokals bewirkt, wurde $[\varepsilon]$ gewählt. Der stimmlose dentale Frikativ [ $\theta$ ] kann, anders als im Englischen, problemlos 
durch den alveloaren Frikativ [s] ersetzt werden, da dieser insbesondere für das südamerikanische Spanisch verzeichnet ist.

Im Fall der finnischen Gemeinde Ähtäri werden die Phoneme, die im deutschen Lautsystem nicht vorhanden sind, durch solche ersetzt, die ihnen auditiv am nächsten sind, d. h. fast offenes [æ] durch halboffenes $[\varepsilon]$. Der stimmlose glottale Frikativ [h] ist im Deutschen zwar vorhanden, allerdings nicht zwischen Vokal und Konsonant. Hier findet, bedingt durch die komplementäre Distribution von $[c ̧] /[\mathrm{x}]$, eine Eindeutschung zu dem stimmlosen palatalen Frikativ [ç] statt.

$\mathrm{Zu}$ Abweichungen der Angleichungsregeln kann es insbesondere bei Personennamen kommen. Diese werden, nach Möglichkeit, auf die jeweilige Person bezogen recherchiert. Beispiel hierfür sind zwei Mitglieder aus dem Adelsgeschlecht Dohnánji (ungarisch: ['dohna:nji]). Der SPD-Politiker und ehemalige Erste Bürgermeister von Hamburg, Klaus von Dohnányi, bevorzugt die Aussprache [,klaus fon do'na:ni], sein Bruder, der Dirigent Christoph von Dohnányi, präferiert [, kristof fon 'doxna:nji].

\subsection{Transkriptionssysteme in der ADB}

\subsubsection{IPA}

Zur normphonetischen Transkription nutzt die ADB das Internationale Phonetische Alphabet (IPA). Zusätzlich kommt noch eine vereinfachende Umschrift zum Einsatz (siehe 3.3.2). Jeder Eintrag erhält außerdem noch ein von Sprecher*innen vertontes Audiofile.

Die IPA-Transkription wird auf der Basis der Duden-Transkription von Max Mangold (2005) angegeben. Einige Merkmale, die in der ADB Anwendung finden, sind:

- Diphthonge sind mit Ligaturbögen unten markiert, d. h. [ạu], [ại], [əy],

- /r/ für dt. Allophone [R], [в], [r] sowie engl. [..],

- Betonungszeichen vor der betonten Silbe, Nebenbetonungen möglich. 
Die Orthographienähe der Mangold-Transkription wird von den Redaktionen geschätzt und ist dort gut etabliert. Bei lautähnlichen Diphthongen in anderen Sprachen wird, sofern es sie gibt, die kodifizierte Norm der Gebersprache verwendet. Englische Diphthonge werden daher nach Jones (2011) bzw. Wells (2008) als [av], [ar], [गI], etc. transkribiert. Für Anwender*innen ist dies auch ein Marker, dass der Begriff nicht deutsch zu sprechen ist.

\subsubsection{Vereinfachende Umschrift}

Neben der IPA-Transkription werden Angaben zur Aussprache auch in einer vereinfachenden Umschrift dargestellt, um den Anwender*innen, die mit der IPA-Notation weniger vertraut sind, eine Hilfestellung zu geben. Ein weiterer, eher pragmatischer Grund, ist die schnelle Versendung von Recherche-Ergebnissen per E-Mail, da die korrekte Darstellung der IPA bei den Empfänger*innen nicht immer gewährleistet ist.

Zur Veranschaulichung der Umschrift sind nachfolgend einige Merkmale aufgeführt:

- Vokallänge wird mit nachfolgendem „h“, Vokalkürze mit nachfolgender Konsonantenverdoppelung markiert:

-Verona - IPA: [ve'ro:na] - Umschrift: We'rohna

- Donald Trump - IPA: [,dpnəłd 'trımp] - Umschrift: ,Donnelld ,Trammp

- Markierung von Stimmhaftigkeit/-losigkeit sowie Nasalierung über Hinweise in Klammern:

- Visegrad - IPA: ['vIfEgra:t] - Umschrift: ,Wischäggraht (sch=stimmlos)

- Lens - IPA: [lã:s] - Umschrift: Lahs (a=nasal, s=scharf)

- Ithaca (USA) - IPA: ['IOəkə] - Umschrift: ,Ithekke (th=engl., stimmlos)

- Unsilbische Vokale in betonten Silben: Betonungszeichen vor dem silbentragenden Vokal:

- Chiara - IPA: ['kia:ra] - Umschrift: Ki'ahra 
- „Vokalisiertes R“: nach kurzem Vokal „a“, nach langem Vokal „““:

- Hundertwasser - IPA: ['hondetvase] - Umschrift: ,Hunndattwassa (ss=scharf)

- Gewehr - IPA: [gə've:en] - Umschrift: Ge'wehr

\subsection{Phonemvariation in der ADB}

Die aktuelle Auflage des Duden-AWB (2015) bildet Phonemvariation in stärkerem Maße ab als vorherige Ausgaben, da ,sowohl national als auch großregional gebräuchliche subnationale Varianten berücksichtigt werden und auch der Standardsprachgebrauch von Sprecher(inne)n ohne spezielle Ausspracheschulung bzw. Sprechausbildung einbezogen wird“ (S. 22). In der ADB findet eine geringere Abbildung von Phonemvariation statt, da bei den Anwender*innen der Wunsch nach Eindeutigkeit besteht, gleichwohl werden regionale Aussprachevarianten angegeben, insbesondere wenn dies von Rundfunkanstalten für ihr jeweiliges Sendegebiet erwünscht wird. Als Beispiel seien hier die nordrhein-westfälischen Städte Telgte und Lügde aufgeführt, die überregional ['telktə] bzw. ['lyktə] ausgesprochen werden, in Teilen des Sendegebiets des Westdeutschen Rundfunks jedoch als ['tદlçtə] bzw. ['lyçtə]. Exemplarisch für den Bereich außerhalb des binnendeutschen Sprachraums sind die Beispiele China und Venedig, deren Aussprache für das Gebiet des Österreichischen Rundfunks (ORF) als ['ki:na] bzw. [ve'ne:drk] in der ADB verzeichnet sind.

\section{Transkription von Standardaussprache aus Sicht des Faches Deutsch als Fremdsprache (Robert Skoczek)}

\subsection{Phonetische Transkription als Hilfsmittel}

Schon ab der ersten Unterrichtsstunde tauchen die Sprachlernenden in die neue Sprache ein. Der handlungsorientierte Ansatz zielt auf die Aktivierung der Lernenden ab, sodass die klassischen Fertigkeiten Hören, Sprechen, Lesen und Schreiben trainiert werden. Der fremde Sprachklang wird dadurch mit einem neuen Schreibsystem vermittelt. Dabei stützt das Schriftbild über den 
visuellen Kanal oft die Gedächtnisleistung beim Erlernen neuen Wortschatzes, der wiederum schriftorientiert in den sprechsprachlichen Äußerungen der Sprachlernenden zusätzlich durch den muttersprachlichen Filter verformt wird. Die interlingualen Interferenzen wirken gleichzeitig auf verschiedenen Ebenen. Bekannten Schriftzeichen werden vertraute Lautwerte aus der Muttersprache oder den vorher gelernten Fremdsprachen zugeordnet. Es kann zudem oft beobachtet werden, dass Lernende die Aussprache neuer Wörter in das bereits bekannte Schriftsystem transliterieren, um auf diese Weise den gewonnenen Höreindruck des fremdsprachlichen Klangs später in eigenen Sprechtexten wiedergeben zu können. In diesem Zusammenhang stellt sich die Frage, ob eine normphonetische Transkription von Beginn an zur Entwicklung der phonetischen Kompetenz in Deutsch als Fremdsprache Verwendung finden soll. Die Meinungen der DaF-lehrer*innen sind geteilt. Diesem Für und Wider liegen nicht selten methodisch-didaktische Defizite und fehlendes Fachwissen aufseiten der Fremdsprachenlehrer*innen zugrunde (vgl. BryłaCruz 2018, 4 f.). Die Kritiker*innen behaupten, dass Deutschlernende mit dem zusätzlichen Schriftsystem unnötig überfordert würden. Der didaktische Prozess werde umso mehr erschwert, je mehr sich das Schriftsystem der jeweiligen Ausgangssprache von der auf dem lateinischen Alphabet basierenden Schreibung des Deutschen unterscheide, wie schon Dieling/Hirschfeld festgestellt haben (vgl. 2000, 41). Die Skepsis ergibt sich auch aus der Unsicherheit der Fremdsprachelehrenden, ob und in welchem Ausmaß deutlich erkennbare Fortschritte in der phonetischen Kompetenz der Lernenden durch Einsatz einer Lautumschrift im Deutschunterricht eintreten.

Es lassen sich allerdings mehr Argumente anführen, die für die Verwendung der IPA-Transkriptionszeichen im Deutschunterricht sprechen:

- In der Tradition des Englisch- oder Französischunterrichts ist die IPA-Transkription wegen der großen Diskrepanz zwischen Schreibung und Aussprache seit langem Gegenstand des Unterrichts und von Lehrmaterialien. Die Ausspracheangaben gelten somit auch als Bestandteil von Lehrmaterialien.

- Deutsch wird oft als zweite Fremdsprache nach Englisch gelernt. Da IPA-Symbole im Englischunterricht regelmäßig verwendet werden, 
sind sie längst schulische Realität und auch Deutschlernenden bekannt.

- Mit der Verwendung der IPA-Transkription in Lehrwerken wird der autonome Wortschatzerwerb sowohl während des Fremdsprachenunterrichts als auch beim selbstgesteuerten Lernen unterstützt. Die oft knapp bemessene Zeit kann stattdessen für Festigungsübungen oder angewandte Sprech- bzw. Hörübungen genutzt werden.

- Die Kenntnis der IPA-Symbole ermöglicht es Deutschlernenden, selbständig mit Referenzwerken wie Aussprachewörterbüchern und Aussprachedaten-banken zu arbeiten, in denen diese Notation verwendet wird.

- Da es sich bei der IPA-Transkription um eine internationale Konvention zur Notation von Lauten handelt, können die Lernenden von diesen Kenntnissen ebenfalls beim Erwerb weiterer Sprachen Gebrauch machen.

\subsection{Komplexität der Phonem-Graphem-Beziehungen im Standarddeutschen}

Obwohl alle europäischen Sprachen auf dem alphabetischen Prinzip beruhen, heißt das nicht, dass die Kenntnis der Buchstaben und der dahinter stehenden Lautwerte ohne weiteres aus der einen in die andere Sprache überführt werden kann. Zwischen Sprachen, deren Schreibung auf dem lateinischen Alphabet basiert, kommt es zu graphemisch-phonischen Interferenzen. Lesen und Schreiben in einer Fremdsprache können zusätzlich durch die Komplexität der Schreibung in der Zielsprache erschwert werden. Die Phonem-Graphem-Beziehungen der deutschen Standardsprache sind sehr komplex. Sie lassen sich nicht in einfache Regeln fassen (vgl. Hirschfeld/Reinke 2016, 83). 


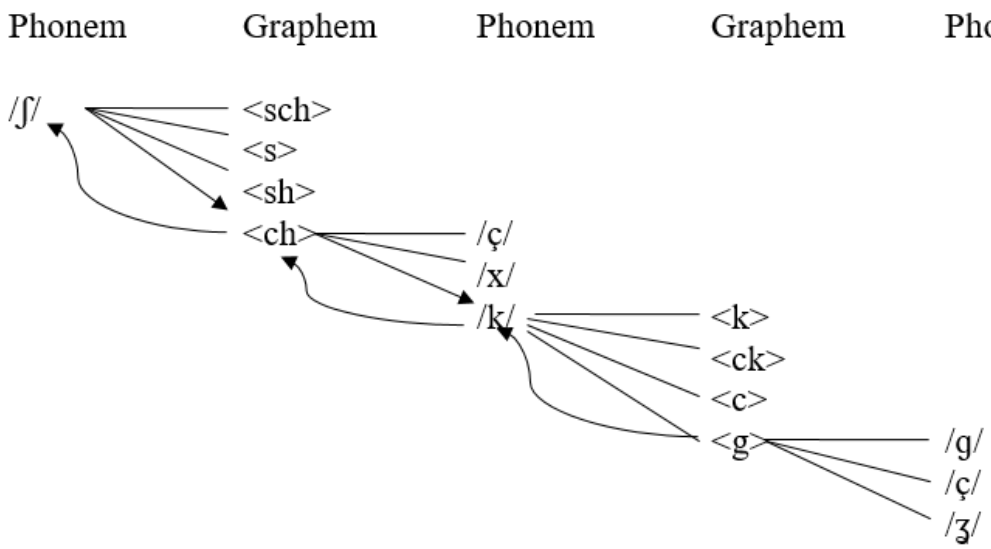

Abb. 4: Interdependenz zwischen der graphemischen und phonologischen Ebene

Die Abbildung 4 exemplifiziert die Komplexität der Phonem-Graphem-Beziehungen für die deutschen Phoneme / $/$ / und / $/ \mathrm{k} /$ und die Grapheme $<\mathrm{ch}>$ und $<\mathrm{g}>$. Der Übersicht ist u. a. zu entnehmen, dass das Phonem / $/$ durch ein einziges Zeichen $(<\mathrm{s}>$ spät) oder eine Zeichenkombination $(<\mathrm{sch}>$ Schule, $<$ sh $>$ T-Shirt oder $<$ ch $>$ Chance) in der Schrift repräsentiert werden kann. Das Graphem <ch $>$ steht außer für / $/$ / stellvertretend für andere Phoneme im Deutschen wie etwa /ç/ (Milch), /x/ (acht), /k/ (Charta). Das Phonem /k/ kann wiederum $u$. a. durch $\langle\mathrm{g}>$ wie in Tag wiedergegeben werden, das ebenso für das Phonem/g/ (Tage) und /ç/ (fleißig) steht. Die Grapheme in der deutschen Rechtschreibung sind somit polyfunktional. Die Korrespondenzen zwischen den Graphemen und Phonemen und umgekehrt sind polyrelational. Diese Polyrelationalität wird in der Abbildung mit den in verschiedene Richtungen gerichteten Pfeilen illustriert. Ein kompletter Überblick über die Phonem-Graphem-Beziehungen im Deutschen wird in Hirschfeld/Reinke (2016, 82 ff.) gegeben und besprochen.

Als Hilfestellung für Deutschlernende wird häufig eine Regel formuliert, laut der ein Stammvokal kurz und ungespannt gesprochen wird, wenn ihm zwei oder mehrere Konsonanten folgen, z. B. Most [most], Herz [he $\left.\varepsilon^{\mathrm{s}} \mathrm{ts}\right]$, Markt $\left[\mathrm{ma}^{\mathrm{b}} \mathrm{kt}\right]$. Die Umsetzung der Schreibsprache in die Sprechsprache erfolgt jedoch nicht nach diesem einfachen Mechanismus. Etymologisch, sprachhisto- 
risch und morphologisch bedingte Abweichungen von der obigen Regel lassen sich bereits im Anfangsunterricht nachweisen, z. B. Prost [рбо: st], werden

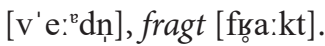

In der Anfangsetappe des Fremdsprachenerwerbs versuchen Sprachlernende, die bereits vermittelten Sprachstrukturen und Vokabeln individuell zu organisieren, um sie sich effektiver und schneller anzueignen. Dabei suchen sie nach Regelhaftigkeit und tendieren zur Generalisierung vermittelter bzw. beobachteter Spracherscheinungen. Dies spiegelt sich in der intralingualen Interferenz wider, auch im phonetischen Bereich. Ausspracheregeln, auf die sich Lernende nicht ganz verlassen können, können verwirrend wirken und viele schwer zu beantwortende Warum-Fragen auslösen.

\subsection{Ausspracheangaben in Lehrwerken}

Phonetik spielt in der Anfangsetappe des Sprachunterrichts eine wichtige Rolle. Allerdings lassen sich wenige Lehrwerke finden, in denen verschiedene Übungsschwerpunkte, methodische Abwechslung und umfangreiches Übungsmaterial angeboten werden. Gerade die jüngsten Deutschlernenden, die sich noch durch Imitation eine fremde Aussprache aneignen können, werden diesbezüglich vernachlässigt. Im schlimmsten Fall werden ihnen sogar fehlerhafte Aussprachemuster vermittelt und der Eindruck gegeben, die Aussprache der Fremdsprache würde sich von der in der Muttersprache kaum unterscheiden.

Die Übungsbücher Niemiecki dla dzieci (Deutsch für Kinder) von Bednarska und Basse (2015) sind ein Beispiel dafür, wie typische phonetische Interferenzfehler eher fossilisiert werden und das phonetische Potenzial Deutsch lernender Kinder nicht ausgeschöpft wird. In den verwendeten Ausspracheangaben der Abbildung besteht beispielsweise kein Unterschied in der Aussprache der folgenden fett gedruckten Buchstaben in Kopfhörer, Wörterbuch, Spülmaschine, Küchenmaschine, Bleistift, Pony. In dieser Position soll laut Bednarska/Basse der Vokal [i] gesprochen werden, der als (y) bzw. /y/ umschrieben wird. Dabei geht es hier um vier verschiedene deutsche Phoneme. Die Autorinnen weisen mit der Verdoppelung der Vokale auf deren Dauer hin. Dies wird zum einen inkonsequent gemacht, was mit dem Beispielwort 
Spülmaschine veranschaulicht wird. Zum anderen können diese Doppelvokale missverständlich wirken, denn im Polnischen stehen Verdoppelungen in der Graphie für zwei Laute.

\section{WÖRTERBUCH wyrtetucht}

\section{SŁOWNICZEK}

Affe, der ( $\boldsymbol{m}$ ) lafe/ małpa

Antilope, die (f) /antiloope/ antylopa

Auge, das (n) latge/ oko

Auto, das (n) /alto/ samochód

Ball, der (m) / bal/ piłka

Bauklötze, die (Pl.) /batklycel klocki

Bleistift, der ( $m$ ) /blajsztyft/ ołówek

Brot, das (n) /broot/ chleb

Buch, das (n) /buuch/ książka
Nase, die $(f)$ /naaze/ nos

Nashorn, das (n) /naazhorn/ nosorożec

Nilpferd, das (n) /niilpfeert/ hipopotam

Notizbuch, das (n) /notiicbuch/ notes

Ohr, das (n) loor/ ucho

Papagei, der (m) /papagaj/ papuga

Papier, das ( $\boldsymbol{n}$ ) /papijer/ papier

Pizza, die (f) /pyca/ pizza

Pony, das (n) / pony/ kucyk

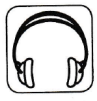

die Kopfhörer

/kopfhyyrer/słuchawki

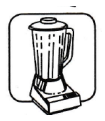

die Küchenmaschine

/kyśenmasziine/ robot kuchenny

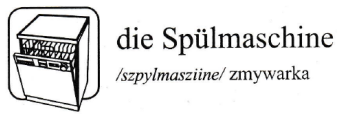

Abb. 5: Transkriptionsbeispiele aus dem Lehr- und Arbeitsbuch Niemiecki dla dzieci 8-10 lat (Bednarska/Basse 2015, 1 u. 13 f.)

Mit solchen Notationssystemen wird eher Schaden angerichtet. Diese notdürftige Transkription beweist, dass Lehrende nach Lösungen suchen und immer noch ein fachlicher und methodisch-didaktischer Fortbildungsbedarf besteht. Es wäre somit ratsam, auf das anerkannte Transkriptionssystem der IPA zurückzugreifen. Die IPA-Transkription sollte normphonetisch regelhaft angewendet werden, wie das u. a. im DAWB (siehe oben) vorgegeben wird. 


\subsection{Arbeit mit der IPA-Transkription}

Die Transkription soll im fremdsprachendidaktischen Kontext nicht l'art pour l'art darstellen, denn die „Transkription hat eine dienende Funktion“(Dieling/ Hirschfeld 2000, 41), d. h., mit deren Hilfe sollen den Lernenden phonetische Unterschiede im Deutschen bewusst gemacht werden, die einzelne Lautsegmente wie auch Lautfolgen betreffen. Aus dem gesamten Inventar von Lautzeichen sollten demnach nur diejenigen Symbole verwendet werden, mit denen typische Erscheinungen der deutschen Standardaussprache veranschaulicht werden können. Deutschlernende sollen sukzessiv im Spracherwerbprozess mit den Lautzeichen vertraut gemacht werden. Hierbei geht es um passive, rezeptive Fertigkeit.

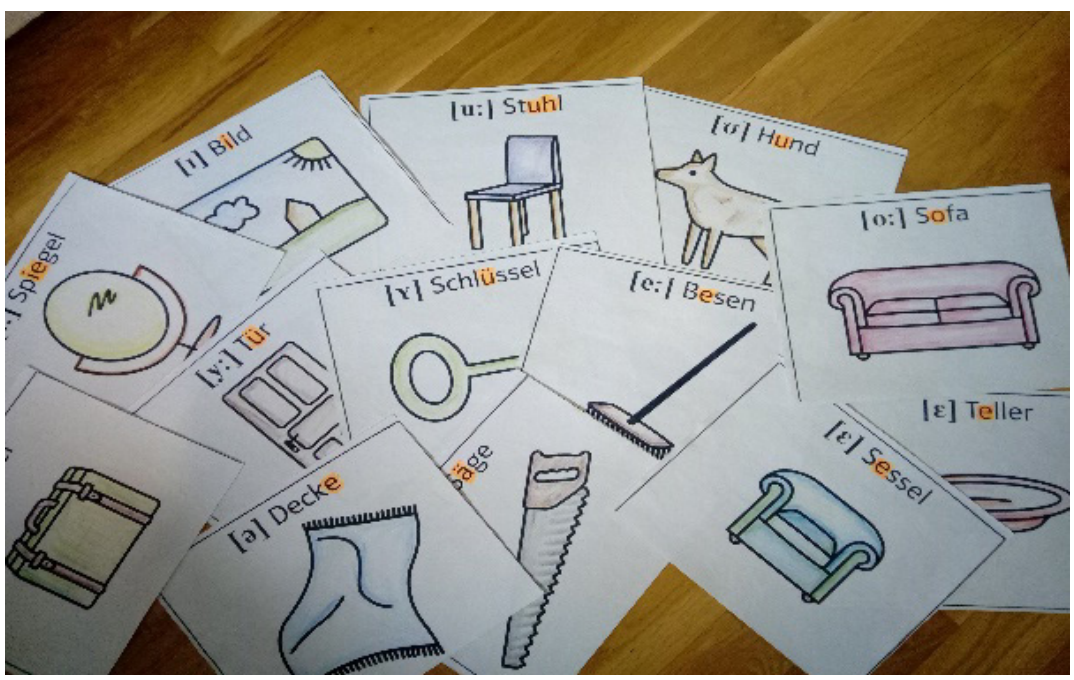

Abb. 6: Bilder mit den bezeichneten Objekten, der orthografischen Notation und dem jeweiligen Lautsymbol

Die obigen Bilder, die aus einem Aussprachespiel (vgl. Hirschfeld/Reinke 2014, 129 ff.) zu diesem Zwecke adaptiert wurden, können beispielsweise immer am Unterrichtsanfang eingesetzt werden. Deutschlehrende zeigen ihrer Lerngrup- 
pe zuerst nur die Bilder, die die Differenzen im deutschen Vokalismus und die Phonem-Graphem-Beziehungen verdeutlichen darstellen. Sie sprechen die Wörter vor und die Gruppe spricht alles nach. Anschließend kann ein Smalltalk geführt werden, der die Lernenden allmählich in das Unterrichtsthema einführt. In den ersten Stunden kann sich der Wortschatz auf Gegenstände im Klassenraum beschränken wie Stuhl, Tafel, Tisch etc. Die phonetischen Bilder lassen sich ohne weiteres in verschiedene Unterrichtsthemen einbinden. Im Unterricht, in dem Lernende ihre Meinung äußern lernen, kann die Aussprache in Phrasen wie ich meine, ich finde geübt werden. Den Lernenden wird das jeweilige Bild gezeigt und sie werden aufgefordert, auf Fragen zu reagieren z. B. Wie findest du das Sofa? Und wie findest du den Koffer? - Der Koffer ist..., ich finde das Sofa... Bei der Arbeit mit einem Bilderlexikon wie etwa im Lehrwerk Menschen lassen sich ebenfalls Identifikationsübungen durchführen. Deutschlernende suchen in dem neuen Bilderlexikon zu der jeweiligen Lehreinheit die Wörter aus, die denselben Vokal wie die abgebildeten Gegenstände auf den gezeigten Bildern haben. Mit der Zeit können die Bilder mit Gegenständen durch Kärtchen mit IPA-Zeichen ersetzt werden.

\subsection{Fazit}

Die Arbeit mit der IPA-Transkription bietet vor allem Vorteile. Deutschlernende werden von Anfang an für lautsprachliche Unterschiede sensibilisiert und hinterfragen die Verwendung der phonetischen Schrift nicht. Sie gehört zum Deutschunterricht genauso wie Vokalbellernen, grammatische Übungen, Hörverständnisübungen und viele andere Übungsformen. In meiner Berufspraxis als Deutschlehrer wie auch Fortbildner wurde vielfach von Lehrenden bestätigt, dass es gerade in den Anfangskursen viel Zeit, die gut für Ausspracheübungen genutzt werden könnte. Neben der Verdeutlichung der deutschen Phonem-Graphem-Beziehungen, der Darstellung phonetischer Besonderheiten und der Anwendbarkeit der IPA-Transkription beim Erlernen weiterer Sprachen kann die DaF-Unterrichtspraxis methodisch sowohl fundiert als auch aufgelockert werden. 


\section{Literatur}

Bednarska, Joanna/von Basse, Monika (2015): Niemiecki dla dzieci 8 - 10 lat. Pierwsze słówka. Ćwiczenia. Miedzyrzecz: Literat.

Bonner, Maria (2017): Die Neuauflage des Duden-Aussprachewörterbuchs - eine hilfreiche Neuorientierung? In: German as a foreign Language. gfl-journal 2. $<$ http://www.gfl-journal.de/2-2017/bonner.pdf> (18.05.2020)

Bryła-Cruz, Agnieszka (2018): Różne biografie edukacyjne, ten sam cel pragmatyczny. Szczególny przypadek dokształcania nauczycieli języka angielskiego. In: Karolczuk, Joanna (Hg.): Języki obce w szkole, 4. Warszawa: Wydawnictwo FRSE.

Dieling, Helga/Hirschfeld, Ursula (2000): Phonetik lehren und lernen. Fernstudieneinheit 21. Berlin: Langenscheidt Verlag.

Glück, Helmut/Rödel, Michael (Hg.) (2016): Metzler Lexikon Sprache. Stuttgart: Metzler.

Jones, Daniel (2011): English Pronouncing Dictionary. Cambridge: Cambridge University Press.

Hirschfeld, Ursula/Reinke, Kerstin (2018): Phonetik im Fach Deutsch als Fremdsprache. Berlin: Erich Schmidt Verlag.

Hirschfeld, Ursula/Reinke, Kerstin (2014): 44 Aussprachespiele. Berlin: Ernst Klett Verlag.

Kleiner, Stefan/Knöbl, Ralf (Hg.) (2015): Duden. Das Aussprachewörterbuch. Berlin: Dudenverlag.

Krech, Eva-Maria/Stock, Eberhard/Hirschfeld, Ursula/Anders, Lutz Christian (Hg.) (2009): Deutsches Aussprachewörterbuch. Berlin: De Gruyter.

Krech, Eva-Maria/Kurka, Eduard/Stelzig, Helmut/Stock, Eberhard/Stötzer, Ursula/Teske, Rudi (Hg.) (1982): Großes Wörterbuch der deutschen Aussprache (unter Mitwirkung von Jung-Alsen, Kurt). Leipzig: VEB Bibliographisches Institut.

Krech, Eva-Maria/Kurka, Eduard/Stelzig, Helmut/Stock, Eberhard/Stötzer, Ursula/Teske, Rudi (Hg.) (1964): Wörterbuch der deutschen Aussprache. Leipzig: VEB Bibliographisches Institut.

Mangold, Max (Hg.) (2005): Duden. Das Aussprachewörterbuch. Berlin: Dudenverlag.

Muhr, Rudolf (2007): Österreichisches Aussprachewörterbuch/Österreichische Aussprachedatenbank. Frankfurt a. M.: Peter Lang.

Wells, John C. (2008): Longman Pronunciation Dictionary. Essex: Pearson Education Ltd.

Internetquelle 1: https://adbmobile.hr-online.de/adbmobile/\#!/ (letzter Zugriff 2020-03-26)

Internetquelle 2: https://de.forvo.com/about/ (letzter Zugriff 2020-03-26).

Internetquelle 3: http://www.adaba.at/ (letzter Zugriff 2020-03-26). 

Uwe Hollmach, München/Halle (SaAle)

\section{Ausspracheideale heute}

Zusammenfassung: Jede kulturelle Zeitlinie bringt Aussprachevorbilder hervor, die seit dem Ende des 19. Jahrhunderts als Tonaufnahmen vorliegen. Standen in früherer Zeit im Sprechausdruck die Klanggestaltung im Vordergrund, sind es heute eher verschiedene Sprechstile, die im Theater und den Medien zu hören sind und vorbildhaft wirken können. Die verschiedenen Spielarten des standardsprachlichen Gebrauchs gelten als wesentliche Anforderung in der gegenwärtigen Sprechbildung an den Schauspielschulen.

Stichworte: Aussprachevorbilder, Sprechausdruck, Sprechstil, Sprechbildung, Schauspielschulen

Abstract: Every cultural timeline produces ideals of phonetics that have been saved as sound recordings since the 19th century. In former times artificial phonetic sound design was a central aspect, yet there are various speaking styles you can hear on stage and in the media nowadays that may be jugded as exemplary in this context. The development mentioned above has come into focus as a substantial requirement for voice and speech training at artistic schools.

Keywords: ideals of phonetics, artificial phonetic, speaking style, voice and speech training, artistic schools

Inspirieren die Akteure in ihren Aufführungen die Zuschauer oder rühren ihr Herz, dann geschieht dies auf einem künstlerischen Niveau auch über den Gebrauch der gesprochenen Sprache. Die lautsprachlichen Äußerungsmomente sind dabei Teil eines theatralen Prozesses oder wirken - eher in den Vordergrund gerückt - über die elektronischen Medien in Hörbüchern 
oder in Hörspielen. Das sprechsprachliche Geschehen beeindruckt demnach in Verbindung mit einem situativen Konzept, worüber für die Teilnehmer und Teilnehmerinnen eine erfahrbare Gegenwärtigkeit etabliert wird. Im weiteren Textverlauf wird über das generische Maskulinum jedes persönliche Geschlecht angesprochen.

Das Vorhandensein eines situativen Rahmens umschließt die dargestellten Aktionen sowie etwas weiter gefasst deren Beobachtung, welche aus verschiedenen Perspektiven heraus geschehen kann. Sind die Zuhörer in dem Augenblick anwesend, dann erleben sie das Sprechen in einem gegenwärtigen zeitlichen Kontext. Für sie könnte in einem situativen Moment die Äußerung während einer Aufführung an einen hohen Erlebniswert gebunden sein, obgleich bei einer näheren phonetischen Betrachtung die Aussprache weniger orthoepischen Vorgaben folgt. Ebenso vage scheint der Zusammenhang bei einer umgekehrten Sichtweise. Vor allem in theatralen Prozessen trägt eine geschärfte standardsprachliche Abbildung, wie die Lauttrennung oder das artikulatorische Unterstreichen der Verschlusslaute, nicht unbedingt zu einem erhöhten Erlebniswert bei. Bestimmend für eine als vorbildlich empfundene Aussprache ist demnach weniger das schablonenhafte Abbilden einer vorgegebenen Lautungsform als das Erzeugen von Ereignismomenten, die Erlebnismomente auslösen können, zu denen sich dann ein Perzipient qualitativ verhalten kann. Diese Beobachtung besonders aus dem Schauspiel wirft für die Sprechbildung die Frage auf, inwieweit Ausspracheideale eine Orientierung bieten und welche Merkmale als Anhaltpunkte dienen können. Für das Treffen gültigerer Aussagen hierzu ist zu berücksichtigen, dass kulturelle Zeitlinien sich ebenfalls beeinflussend auswirken. Beim Anhören von Tonbeispielen, die in den 20er oder 30er Jahren eingesprochen worden sind, wird der historische Abstand offensichtlich. Die sprechkünstlerischen Entwürfe werden vordergründig von der stimmlichen Kraft sowie der klanglichen Gestalt getragen, wodurch die erarbeiteten Texte aus heutiger Sicht extensiver wirken. Solche Sprechkunstwerke, geschaffen durch prominente Persönlichkeiten wie Josef Kainz, folgen zwar anderen ästhetischen Leitbildern, unter anderem auch in der Aussprache, dennoch war die künstlerische Wertbildung keine andere als im Heute. 


\section{Auditive Perspektiven}

Die sprechsprachliche Kunstwahrnehmung vollzieht sich in einem zeitbezogenen Deutungsraum, in welchem die Beobachter eine perzeptive Nachvollziehbarkeit erfahren. Solche Vorgänge erklärt der Neurowissenschaftler Eric Kandel in seinem Buch ,Das Zeitalter der Erkenntnis: „Die Wahrnehmung von Kunst erfolgt teilweise über Imitation und Empathie; sie beansprucht die Hirnsysteme für biologische Bewegung, die Spiegelneuronen und die Theory of Mind.“ (2012, 519). Demnach entwickelt sich der Erlebniswert durch beobachtete Handlungsvorgänge auf der Bühne, die sprechsprachliche Äußerungen einschließen. Sie berühren in der auditiven Betrachtung durch inhaltlich-gedankliche Darstellungen, aber vor allem durch prosodische Verläufe wie Stimmklang, Rhythmik und Intonation in einer verschränkten Form mit der Lautung. Die eigene Wahrnehmung dazu, im weitergefassten Sinne von Erleben, also ,verstehen' oder , an sich erfahren' im phänomenologischen Verständnis als die Differenz von Wahrnehmen und Erfahren dieses Wahrnehmens, lässt die Ereignisse zum Erlebnis werden (vgl. Roselt 2005, 149ff.). Diese Vorgänge vollziehen sich in ihrem Geschehen zeitunkritisch, aber die daran gebundenen auditiven Bewertungen folgen einem Zeitgeist, der auch Aussprachevorbilder hervorbringt.

Liegen einige historische Tonbeispiele zum Hören bereit, um sie untereinander gegenüberzustellen, dann würde wahrscheinlich ein erstes Hören wenig Unterscheidbares hervorbringen, weil die sprechsprachlichen Mittel anders als im Heute fokussiert sind. In einer gegenwärtigen Betrachtung zeigen sich eventuell selbst Studierende der Sprechwissenschaft oder des Schauspiels in ihrer ersten auditiven Begegnung mit Josef Kainz oder dem halleschen Sprechkundler Richard Wittsack irritiert, empfindet man doch bisweilen die Sprechentwürfe als unnahbar oder nicht zeitgemäß; die Vorbildhaftigkeit historischer Sprechfassungen bliebe dadurch verdeckt und daher unterschätzt. Besteht in einem Deutungsraum eine zu große historische Distanz zwischen Hörer und Darsteller (Sprecher), dann können Barrieren entstehen, die den Erlebniswert und die Interpretationskompetenz schwächen. Diese Höreindrücke resultieren wohl aus dem Gesagten, aber sie ermöglichen zudem einen Rückblick auf die Erarbeitungsweisen von Textvorlagen oder schauspielerischen Figuren, die 
auch sprachlicher Art sind. Ebenso werden die folgenden Generationen von auditiven Betrachtern aus ihrer kulturellen Zeitlinie heraus zu Einschätzungen gelangen, deren Beschreibungen anders ausfallen werden als die aktuellen Aussagen dazu.

Eine Annäherung an sprechsprachliche Vorbilder erfordert offensichtlich variable Hörweisen, die sich in einem musikalisch-sprachlichen Kontinuum bewegen. Für das Sich-Einhören in eine historische Sprechfassung ist zudem offenbar eine andere körperliche Bereitschaft nötig, um den Sprecher, wie es der Germanist Richard Benz 1926 feststellt, in seiner leiblichen Gegenwart in dem eigenen seelisch-körperlichen Vorstellen erfahren zu können (vgl. S. 5). Inwieweit sich der Zuschauer während einer Aufführung körperlich von den Affekten über den sprechsprachlichen Ausdruck und die Stimmkraft sowie den Habitus mittragen lässt, ist situativ bestimmt, von dem Genre und dem vorherrschenden Zeitgeist abhängig, worunter die gesellschaftliche Bewertung von Gegebenheiten, Fragen und Lebensstilen verstanden werden kann.

\section{Historische Aussprachevorbilder}

Einen Weg zur näheren auditiven Bestimmung von Aussprachevorbildern bietet der Vergleich von Kritiken und Expertisen aus der jeweiligen Epoche. Für die sprechwissenschaftlichen Nachforschungen kann es als Glücksfall angesehen werden, dass derartige Aufzeichnungen von Sprechkundlern, so die ältere Bezeichnung, beispielsweise von Josef Dischner vorliegen. Seine Lehrer stehen als große Namen für den Beginn unserer Fachgeschichte. Es waren Ewald Geissler (1880-1946), der erste Lektor für Vortragskunst 1905 an der Universität in Halle, und Erich Drach (1885-1935), zweiter begründete die Sprecherziehung als eigenständige Disziplin. Josef Dischner pflegte einen Briefwechsel mit der prominenten Sprechwissenschaftlerin Irmgard Weithase (1906-1982). Mit seinen Anmerkungen bediente er den Wunsch von Irmgard Weithase, als fachkundiger Ohrenzeuge das vorbildhafte Ausspracheverhalten berühmter Schauspieler zu skizzieren, von denen keine oder nur mangelhafte Tonaufnahmen vorliegen konnten. So erlebte er Josef Kainz zu seiner Glanzzeit 1893 mit einer Aufführung im Deutschen Theater. Sein mimisch-sprachli- 
cher Ausdruck auf einer späteren Tonaufzeichnung von 1908 mit dem Hamlet-Monolog von William Shakespeare entsprach nach Josef Dischners Aussage jedoch keineswegs seiner wahren Meisterschaft. Beim gegenwärtigen Hören beeindruckt das Ereignis dennoch durch eine hohe Dichtheit in der sprechsprachlich-körperlichen Übertragung des dramatischen Textes.

In der damaligen Theaterwelt ebenfalls prominent vertreten war der Schauspieler Ernst von Possart, der als erster Intendant des Prinzregententheaters in München (1901) über größeren Einfluss verfügte, der auch im Zusammenhang mit der Ausspracheregelung steht. Was er unter einer vorbildhaften Aussprache versteht, findet man in seinem Büchlein ,Die Kunst des Sprechens' von 1909 beschrieben, das auf seine 40 -jährige Erfahrung und Beobachtung gründet. Er wendet sich mit den einzelnen Lautbeschreibungen und Wortlisten vor allem an Berufsredner und Bühnendarsteller. Bemerkenswert ist die Perspektive, aus der heraus die Lautbeschreibungen und Sprechübungen erklärt werden. Sie sind stets an ein rhythmisches Verständnis gebunden. Jedes Wort wird mit einer fortlaufenden silbischen Markierung ,schwer-leicht' versehen. Ernst von Possart forderte eine getrennte Einzellautbehandlung gleicher Verschluss- und Reibelaute an Wortgrenzen z. B. und | der, auf | Vorteil bedacht. Wohl auch deshalb wirkt sein eigener Sprachgebrauch in einer Tonaufnahme überartikuliert, wo er den ,Handschuh` von Friedrich Schiller vorträgt.

Die Kritiker aus der damaligen Presse weisen darauf hin, dass Ernst von Possart selbst wenig vorbildhaft sprach, war doch sein melodisch-rhythmischer Sprechstil maßlos überzogen. Auch der Sprechkundler Josef Dischner erlebte Ernst von Possart als Darsteller. Er notierte dazu für Irmgard Weithase in einem Brief:

„Possart hatte niemals eine herrliche Stimme [...] Possart sprach und spielte immer gleich wie eine Spieluhr. Er hatte auch kein reines S. Aber - er war ein Meister der gründlichen Durcharbeitung seiner Rollen, die er mit größter Klarheit gliederte und mit größter oft übertriebener Deutlichkeit zum Vortrag brachte. Als Rezitator [...] war er unerträglich affektiert. Das Publikum liebte ihn wegen seiner Klarheit, Deutlichkeit und Effekthascherei." (Dischner 1944) 
Bei einem Wechsel der Position hin zur Lehre der damaligen vorbildhaften Bühnenaussprache wird das Üben an den sprachlichen Mitteln nochmals deutlich. Der prominente Lehrer an der Schauspielschule in Wien, Ferdinand Gregori (1870-1926), verfasste einen Aufsatz, in dem er Einsichten in den sprecherzieherischen Unterricht gibt und Einblicke in die Anforderungen dazu gewährt. „Und glücklich, wenn es so viel sinnliche Erinnerungskraft hat, dass es ihn schon beim bloßen L und M wie Zärtlichkeit und Kussgefühl durchrieselt, [... ]“. (S. 47). Er lehrt die sinnliche Formung von Klanggestalten und bindet damit die Lautäußerungen an ein körperliches Erleben. Seine Schüler üben den Silbenklang im Wechsel von Dur und Moll. Genau diese Art von Lauterziehung fördert jene stimmlich-klanglich angelegte Ausdrucksweise, die für das schauspielerische Sprechen zu seiner Zeit als mustergültig galt. Mit den Übungen zum Absetzen von nebeneinander liegenden betonten und unbetonten Silben werden Notenwerte geformt, die im Verhältnis von einer Viertel zu einer Achtelnote stehen. Darin und in der Melodiebewegung sieht Ferdinand Gregori den wesentlichen Unterschied zur Sprache des allgemeinen Umgangs. Für die Berliner im Alltag würden zwei oder drei Töne ausreichen. Eine solche Melodielosigkeit auf der Bühne würde für das künstlerisch eingestellte Publikum zur Langweile führen, schreibt er (vgl. S. 48).

$\mathrm{Zu}$ dieser Zeit war das Sprechen auf der Bühne in der Textbehandlung und in ihrer sprechsprachlichen Ausformung klar von der Vortragskunst abgehoben, denn anders als in der Gegenwart waren die Sprecher oft keine Schauspieler. Das Nachschaffen eines Dichtertextes als Schallgestalt durch einen Sprecher wurde quasi als heiliger Vorgang betrachtet, der mit großer Ernsthaftigkeit zu vollziehen war, wie es auch der Sprechkundler Ewald Geißler forderte (vgl. 1926, 148). Vergleichend zum Schauspiel schreibt Richard Benz „Dafür erschließt aber die Kunst des Sprechers eine Welt, die höher und bleibender ist, als die kurze Stunde eines fragwürdigen Stückes Wirklichkeit, [...]“ (1926, 9).

Begibt man sich in den Archiven an der halleschen Sprechwissenschaft auf die Suche, um den Weg sprechkünstlerischen Wirkens nachzuzeichnen, dann wird diese Recherche zu einer Begegnung mit vorangegangenen Generationen des Faches. Von den alten Tonträgern schallen die Stimmen von Hans Krech (1914-1961) oder seinem Lehrer Richard Wittsack, welcher bis 1952 das Institut in Halle leitete. Durch das kontinuierliche Aufnehmen, teilweise beginnend 
mit den Bewerbungsaufnahmen für das Studium, lassen sich Sprecherbiographien akustisch nachvollziehen und somit deren Entwicklung abbilden. Einen Einblick dazu bietet der Artikel von Hans Krech aus dem Jahre 1956, der seinen Lehrer Richard Wittsack als Sprecher vorstellt, den er selbst von 1934 bis 1952 hören konnte - die Weltkriegszeit ausgenommen. Die zugänglichen Tonaufnahmen erlauben zudem einige Anmerkungen des Verfassers auf die Wirkung mit dem Abstand zum Jetzt. Verschiedene Quellen belegen das öffentliche Auftreten von Richard Wittsack seit 1910 mit Rezitationen oder das nachschaffende Gestalten des Melodramas ,Die Wallfahrt nach Kevlaar' von Heinrich Heine. Ein damaliger Kulturkritiker beklagte die zu stark dramatisch betonten lyrischen Texte, die er aus diesem Grund als verdorben bezeichnete. Dagegen lobte er die reine Klangfarbe des Organs und die hinreißende Kraft bei dramatischen Textvorlagen. Auffällig ist, wie aufmerksam solche sprechkünstlerischen Vorträge von der Presse, so von dem eher kleinen Köthener Tageblatt, beachtet und besprochen worden sind. Sie zeugen von dem Stellenwert, dem diese Auftritte zukamen. Informativ aus heutiger Sicht ist deren kritische Haltung gegenüber einigen Präsentationen, demnach wurde nicht jede in dem Schallarchiv aufzufindende Fassung in jener Zeit als gelungen angesehen, was bei einer gegenwärtigen Bewertung zu berücksichtigen ist.

Hans Krech umreißt die Sprechweise von Richard Wittsack mit stimmlich bedeutsamen Anmerkungen zu dessen Körperlichkeit. „Der kräftig entwickelte Thorax gab seiner Stimme die ergiebige Atemkraft. Der gedrungen den Schultern aufsitzende Hals zeigte einen großen Kehlkopf, dem sich ein weiträumiges Ansatzrohr anschloss." (Krech 1956, 421). Zur prosodischen Gestaltung schrieb er: „Das leiseste Piano, ebenso das Forte eines Affektausbruches standen zur Verfügung, wenn auch mit Neigung zur Kraftentladung. Die Klangfarbe trug mehr Dur-Gepräge [...].“ (S. 424). Sein Sprechstil verhielt sich wie Kammermusik zur Symphonie, die Sprechweise des Tonfilms zu der des Theaters. Diese Beschreibungen künden bereits eine Zeitenwende im sprechsprachlichen Gestalten an, die dann in den 60er Jahren in eine reduzierte Darstellungsart mündete. Richard Wittsack ermunterte noch zu jener Zeit die Studenten, seine Sprechfassungen nachzuahmen; er sah sich als Ausspracheund Gestaltungsvorbild und galt offenbar auch als solches. 
Um einen Vergleich von damaliger und heutiger Reflektion herstellen zu können, wird exemplarisch eine auditive Beschreibung durch den Verfasser vorgenommen, selbstverständlich nicht mit dem Anspruch der Allgemeingültigkeit. Die Perspektive des Verfassers ergibt sich aus den Tätigkeiten als Sprechwissenschaftler an der Martin-Luther-Universität Halle-Wittenberg im dortigen Fachseminar und an der Theaterakademie August Everding in München im Studiengang Schauspiel.

Richard Wittsack spricht eine Passage aus dem Alten Testament dramatischen Inhalts (Auszug): „König Belsazar machte ein herzlich Mahl seinen Gewaltigen und Hauptleuten/und soff sich voll mit ihnen." (Die Ganze Heilige Schrift 1690, 139). Die bedrohliche Grundstimmung entsteht vornehmlich über die silbisch-rhythmisierte Sprechweise mit einer ganzkörperlichen Anbindung, wodurch die Abbildung der ausschweifenden Geschehnisse in ihren opulenten Bildern gelingt. Er spricht in großen Bögen. Der kraftvolle stimmliche Gebrauch zieht den Hörer in die Gesamtsituation hinein. Einzelne sprachliche Bilder werden über sehr auffällige klangliche Formen voneinander abgesetzt, weniger durch Sprechgeschwindigkeitswechsel oder durch Pausensetzungen. Der durchgängig ästhetisierte Stimmgebrauch, der eher tönt als Handlungen (Gestus) widerspiegelt, überdeckt damit eine differenzierte Entfaltung des Inhalts. Dennoch wirkt die Darstellung der Gesamtsituation auf den Hörer sehr direkt. Der sogenannte direkte Ton gilt gegenwärtig als qualitatives Merkmal. Er steht für eine große Klarheit in der Ansprechhaltung, die die geistig-körperlichen Impulse in Äußerungsimpulse überträgt.

In einer weiteren Tonaufnahme rezitiert Richard Wittsack, Wanderers Nachtlied' von Johann Wolfgang von Goethe. Seine Fassung wirkt aus heutiger Perspektive sehr modern, weil er einen persönlichen Ton dafür wählte. Anders als in dem an einen bestimmten Raum angebundenen Entwurf des religiösen Textes wendet er sich nun bewusst über das Mikrofon an seinen Hörer. Solch ein Umgang mit den technischen Möglichkeiten war zu dieser Zeit ungewöhnlich. Die Gestaltung des Goethe-Gedichtes besticht durch die ausgewogen verwendeten sprecherischen Mittel: fluktuierende Tempi, Pausensetzungen und die behutsam geführte Sprechmelodie. Die imaginierten Bilder, Vorgänge und Teilstimmungen entstehen während des sprechsprachlichen Gebrauchs, sie wirken sehr gegenwärtig und nicht vorgefertigt. Er eröffnet 
dem Hörer durch das partnerzentrierte Vortragen einen Raum, in dem er das Wahrgenommene erleben kann. Das Mikrofon fungiert nicht einfach als Zeuge des Sprechvorgangs, es dient nunmehr als Medium vom Sprecher zum Hörer hin, mit dem erster ganz bewusst den Kontakt sucht. Daraus entwickelt sich ein zurückhaltender intensiver Sprechstil, der über eine persönliche Ansprechhaltung den einzelnen Hörer teilhaben lässt, ihn quasi fast mit seinem Atem berühren kann. Auch die Stimmgebung ist durch den geringen Abstand von nicht viel mehr als $30 \mathrm{~cm}$ eher sonor, nicht vergleichbar mit der Stimmgewalt eines Bühnenvortrags zu dieser Zeit.

\section{Vorbildhafte Aussprache}

Von den veränderten Sichtweisen und Bewertungen in einem fortlaufenden gesellschaftlichen und kulturellen Geschehen wird auch die sprachstilistische Formung erfasst. Die sprechsprachlichen Mittel verteilen sich auf andere Weise im künstlerischen Gebrauch als in den historischen Tonaufnahmen. Auch in der Gegenwart bringt die aufgeführte Sprache Vorbilder hervor, die zudem Lehrergebnisse der geschulten Stimme und Aussprache an den Schauspielschulen sind, die dann wiederum multiplikativ wirken. Eine als ideal wahrgenommene Aussprache ist nicht das Resultat einer korrekten Abbildung einer orthoepischen Kodifizierung, die wohl in der heutigen Zeit auch ein Algorithmus erreichen würde. Wenngleich die Verwendung der deutschen Standardaussprache in theatralen Prozessen sowie in den elektronischen Medien als erstrebenwert erachtet wird und deshalb dominant ist, so ist das Erleben eines Ideals doch vor allem an Momente gebunden. Solche Momente ergeben sich aus der dynamischen Behandlung der Sprache während der Darstellung eines Geschehens, das intentional und emotional perzeptiv gespiegelt wird. Die erlebbare Dynamik in der Aussprache entsteht aus der körperlichen Reaktion auf einen Partner oder aus einer aufgeschriebenen Handlung, die sich auf einen Text bezieht. Der Moment ist nicht nur eine temporale und qualitative Abstimmung des Sprechers mit dem Hörer. Er erfasst den Schauspieler oder den Sprecher während seiner Äußerung. ,Im Moment sein bedeutet im Schauspiel, dass der Akteur eine Wahrnehmung als körperlichen Ablauf 
vollzieht, einschließlich des Atems. Durch den Handlungswillen des Akteurs am Partner schreibt das Gehirn ständig neue Sequenzen einer Beobachtung; die körperlichen Reaktionen darauf schließen wiederum an das Sprechen an. Die Aussprache wirkt im Moment nachvollziehbar und ist von einem hohen Erlebniswert getragen. Im Schauspiel sowie in der Sprechwissenschaft wird diesen perzeptiv-körperlich nachvollziehbaren Äußerungsmomenten eine Transparenz oder, gleichbedeutend, eine Durchlässigkeit zugeschrieben, da hierdurch eine Imagination mit der Sprechsprache für die individuelle Deutung differenziert möglich wird. In der Stimm- und Sprechbildung wird eine solche gestische handlungsorientierte Sprache angestrebt, deren Beherrschung vorbildlich sein kann. Dem entgegen kann eine mangelnde körperliche Flexibilität stehen oder eine statische bildhafte Vorstellung von der Handlung. Der Akteur wäre sich zwar darüber im Klaren, was er dem Partner vermitteln möchte, er überträgt diesen Handlungsaspekt aber nicht als sequenziellen Ablauf. Die Aussprache wäre wohl phonetisch korrekt, würde jedoch in einem Zustand verharren, ohne über einen gestischen Gehalt zu verfügen.

Diese körperliche Anbindung im Handeln stellt einen Bezug zur Aussprache her, der unmittelbar an die phonetische Sprechsilbenstruktur des Deutschen gekoppelt ist. Durch die gestische körperliche Dynamik werden rhythmisch-melodische Verläufe als prosodische Mittel auf den Sprechfluss übertragen und verschränken sich mit der Lautungsebene. Über die Rhythmik erhält der Silbenanlaut eine Markierung, die für die Sprechverständlichkeit von Bedeutung ist. Die artikulatorische Bewegung zur lautlichen Äußerung unterliegt ebenfalls der rhythmischen Dynamik. Zur Erhöhung der Ausspracheverständlichkeit beim Singen rücken vor allem die Lautübergänge (Transienten) in den Fokus, weil beispielsweise die Plosive weniger durch das Herausstellen des Ziellautes /p, t, k/ erkannt werden als an den Lautübergängen.

Der rhythmisch-melodische Handlungsmechanismus lässt sich besonders gut an Interjektionen, wie bei $<$ m $>$ oder $<$ ah $>$ erkennen und üben, da hier der Partnerbezug über den rhythmisierten Tonfall beeinflusst werden kann. Ein akustisches Beispiel hierfür wurde mit dem Tagungsvortrag in Halle vom November 2019 vorgestellt. In einer Szene (Synchronfassung) aus dem Film ,Fluch der Karibik I' begegnen sich der Pirat Hector Barbossa und Captain Jack Sparrow in einem Fechtduell. Barbossa richtet sich mit folgender Frage 
an seinen Partner: „Und was jetzt, Jack Sparrow? Sind wir Zwei unsterblich in einen ewigen Kampf verstrickt bis zum jüngsten Tag beim Klang der Posaunen? Hmm?" Über die Interjektion $<$ Hmm? > verkürzt er den Abstand zu Jack Sparrow. Die melodische Interjektionslinie entspricht dabei der Silbenstruktur des Deutschen. Über die letzte angehängte Achtelnote auf den Ton h mit dem Sprung ins Falsett wird ein starker Handlungsimpuls erzeugt, durch den Jack Sparrow quasi zu einer Reaktion genötigt wird.

\section{Jack Sparrow /Barbossa}

Frage-Interjektion

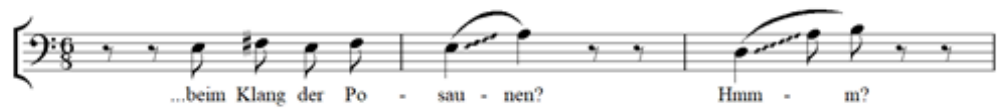

Abb. 1: Notation der Sprechpassage des Hector Barbossa aus dem Film ,Fluch der Karibik

Die kleinste rhythmische Einheit im Deutschen ist die Sprechsilbe und sie ist somit der wichtigste Einzelbaustein, mit dem Handlungen dargestellt sowie Emotionen übertragen werden können. Über die verschiedenen rhythmisch-melodischen Anbindungsweisen der Silben miteinander entstehen zudem verschiedene Sprechstile.

Die nebeneinander anzutreffenden zahlreichen Spielarten theatraler Prozesse bzw. Genres (Performance, Film, Synchronsprechen) bringen neue Sprechstile hervor, wie die stilisierte Alltagssprache mit einer auffällig hörbaren Nähe zur Sprache des täglichen Umgangs, ohne jedoch ein identisches phonetisches Abbild davon zu sein. Dieser Sprechstil geht auf das Streben zurück, sehr persönlich wirkende Haltungen und Vorgänge über einen dazu passenden Sprechton erzeugen zu wollen. Es ist vor allem der Assimilationsgrad (Lautangleichung), der der Spontansprache ähnelt. Die größten Übereinstimmungen bestehen in der Realisierung des Schwa-Lautes [ə] in den Suffixen -el, -en, -em zu assimilierten Varianten und die Realisierung von auslautendem [t] als Elision (z. B. nicht). Auf der prosodischen Ebene sind die Schwankungen im Tonhöhenverlauf weniger sprunghaft als in der Spontansprache. Die 
Pausen werden zwar bei der gebundenen Sprache mit Abstand am häufigsten gesetzt, doch auf Platz zwei folgt die stilisierte Alltagssprache weit vor der Spontansprache und dem Verlesen eines Sachtextes. Dagegen zeigen im Grad der Sprechspannung die Spontansprache und stilisierte Alltagssprache ähnliche Werte (Hollmach 2013, 250ff.). Unterschiede bestehen zudem in der rhythmisch-melodischen Anbindung der Sprechsilben. Liegt die Schwere in der gebundenen Sprache eher im Zentrum der betonten Silbe, so erfasst dieser Schwerpunkt in der stilisierten Alltagssprache besonders die folgenden Laute, wodurch die nächste Silbe angeschlossen und aktiviert wird. Selbst William Shakespeares ,Richard III' ließe sich so in einer alltagssprachlichen Stilistik spielen „Nun ward der Winter unseres Missvergnügens [...]“.

Sich in verschiedenen Stilen auf verständliche Weise bewegen zu können entspricht einer hohen Sprechkultur, die vorbildhaft ist. Ein solch breites Spektrum in verschiedenen Genres bedienen die Synchronsprecherin und Schauspielerin Daniela Hoffmann oder der Sprecher und Schauspieler Christian Baumann. Er beherrscht diese rhythmisch-melodische Klaviatur, sich in verschieden Stilen verständlich und gestisch auf sehr hohem Niveau äußern zu können. Seine Wandlungsfähigkeit ist meisterhaft, so im: Unterbildtextsprechen, Hörspiel, Film mit regionalen und ohne regionale Merkmale. Ob es nun eher zufällig ist oder eine Entwicklung anzeigt, dass die Aussprachevorbilder oftmals aus dem süddeutschen bairischen Raum stammen, bleibt hier spekulativ. Auch die Gewährsperson für das Hallesche ,Deutsche Aussprachewörterbuch' Frank Manhold ist eine langjährige Stimme des Bayerischen Rundfunks mit süddeutschen prosodischen Eigenarten, wenngleich in geringer Ausprägung. Die Position als Modellsprecher wurde über eine soziophonetische Untersuchung mit einem umfangreichen Hörkonzept überregional bestimmt. Beide Sprecher verwenden aufgrund ihrer Herkunft einen Sprechstil, der in dem rhythmisch-melodischen Verlauf eher durch Eigenarten, wie ,legato ‘ anstelle von ,staccato ' wirkt (vgl. Hollmach 2007, 237ff.).

Für die Lehre können solche Aussprachevorbilder durchaus als Vorlagen dienen, wenn man sie als eine Orientierung begreift, der man nicht imitativ folgt. Zudem steht davor die Frage, woraus sich die Anerkennung von Sprecher- bzw. Schauspielerpersönlichkeiten erklärt. Diese Frage erlangt einen pragmatischen Charakter, wenn man sich für seine Eleven im späteren Be- 
rufsleben eine ähnliche Beachtung wünscht. Es ist der Erfolg, der auf Können und Qualifikation beruht. Solche fast tugendhaften Merkmale verstehen die Studentinnen und Studenten im Schauspiel sehr wohl als Ansporn, der nach meinen Erfahrungen die Spracheinstellung fördert. Um mit diesem eigenen Anspruch als Stimm- und Sprechbildner unterrichten zu können, bedarf es einer analytischen Sicht auf die hohen Buchungsraten von erfolgreichen Schauspielern unter anderem in Sprecheragenturen. Gegenwärtige Beobachtungen dazu lassen auf eine situative Wandlungsfähigkeit schließen, die wiederum auf das Herstellen verschiedener Sprechstile beruht. Um daran in der Aussprachlehre für theatrale Prozesse anschließen zu können, sind soziophonetische Untersuchungen empfehlenswert, die beispielsweise auf Ergebnisse zum situativen Sprechen bei einer hohen Verständlichkeit zielen. Die sprechwissenschaftliche Sicht hierbei ist, die Erkenntnisse in den Lehrprozess individuell angepasst in nachvollziehbare Äußerungsmomente für einen hohen Erlebniswert des Publikums überführen zu können.

\section{Literatur}

Benz, Richard (1926): Schauspielkunst und kultische Sprechkunst. In: Geißler, Ewald (Hg.): Der Schauspieler. Berlin: Bühnenvolksbundverlag, S. 3-10.

Die Ganze Heilige Schrift (1690): Martin Luther (Übersetzung). Frankfurt a. M., David Kapitel V.

Dischner, Josef (1944-1945): Briefwechsel mit Josef Dischner aus dem Nachlass von Irmgard Weithase. München.

Geißler, Ewald (1926): Wir alle. In: Geißler, Ewald (Hg.): Der Schauspieler. Berlin: Bühnenvolksbundverlag, S. 147-153.

Gregorie, Ferdinand (1926): Die Vorbildung des Schauspielers. In: Geißler, Ewald (Hg.): Der Schauspieler. Berlin: Bühnenvolksbundverlag, S. 43-50.

Hollmach, Uwe (2007): Untersuchungen zur Kodifizierung der Standardaussprache in Deutschland. Frankfurt a. M.: Peter Lang. (= HSSP 21)

Hollmach, Uwe (2013): Phonostile - Stilisierte Alltagssprache im Theater. In: Bose, Ines/ Hirschfeld, Ursula/ Neuber, Baldur/ Stock, Eberhard (Hg.): Einführung in die Sprechwissenschaft, Tübingen: Narr Verlag, S. 247-252.

Kandel, Erik (2012): Das Zeitalter der Erkenntnis. München: Siedler Verlag.

Krech, Hans (1956): Richard Wittsack als Sprecher. In: Wissenschaftliche Zeitschrift der Martin-Luther-Universität Halle-Wittenberg. Jg. V, Heft 3, Halle/S., S. 421-426. Roselt, Jens (2005): Phänomenologie des Theaters. München: Wilhelm Fink Verlag. 

Julia Kiesler, Bern/Anna Wessel, Halle (SaAle)

Probenforschung im (sprech)künstlerischen Bereich

Zusammenfassung: Der Beitrag beleuchtet zwei Perspektiven der Probenforschung. Im ersten Teil werden forschungsmethodische Aspekte der Probenprozessforschung dargelegt und Theaterproben mit ihren Spezifika als sprechwissenschaftlicher Untersuchungsgegenstand betrachtet. Der zweite Teil widmet sich der (sprech)künstlerischen Probenarbeit und deren Einordnung als künstlerisch-forschende Praxis.

Stichworte: Probenprozessforschung, künstlerische Forschung, Probenpraktiken, Sprechkunst

Abstract: The article highlights two perspectives on rehearsal research. In the first part, methodological aspects of rehearsal research are presented and theater rehearsals and their characteristics are considered as research objects of speech science. The second part is devoted to theater rehearsals and their classification as an artistic research practice with a particular focus on the art of speaking.

Keywords: Rehearsal Research, Artistic Research, Rehearsal Practices, Art of Speaking

\section{$1 \quad$ Einführung}

Sprechwissenschaftliche Untersuchungen im Bereich der sprechkünstlerischen Kommunikation bewegen sich seit jüngster Zeit u.a. im Feld der Probenprozessforschung. Die auch im theaterwissenschaftlichen Kontext noch junge Wissenschaftsrichtung widmet sich der Beobachtung und Aufarbeitung von Probenprozessen unter verschiedenen Gesichtspunkten und Fragestellungen. Probenprozessanalysen mit sprechwissenschaftlichen Forschungsschwerpunk- 
ten setzen aktuell an der Untersuchung interaktiver Aspekte der künstlerischen Probenarbeit am Theater an (vgl. Wessel 2016; 2017) sowie an der Analyse von Texterarbeitungsprozessen in der Zusammenarbeit von Regisseur*innen und Schauspieler*innen in der zeitgenössischen Theaterpraxis (vgl. Kiesler 2016; 2017; 2019; Kiesler/Petermann 2019; Rastetter 2017a; 2017b). Mit ihren interdisziplinär angelegten Forschungsprojekten bildet die sprechwissenschaftliche Probenforschung sowohl auf inhaltlicher als auch auf methodischer Ebene ein Bindeglied zu theaterwissenschaftlichen und auch zu linguistischen Forschungslinien. Ein breiter forschungsmethodischer Erfahrungsschatz existiert bislang noch nicht, jedoch gibt es mittlerweile erste erprobte Untersuchungsansätze und Methodendesigns, mit denen Proben zugänglich gemacht und analysiert werden können (vgl. hierzu Kiesler 2019, 70ff.; Quick 2020; Wessel 2017).

Neben ihrer Bedeutung für die künstlerische Praxis dienen Theaterproben aber nicht nur als wissenschaftlicher Untersuchungsgegenstand, sie werden selbst auch als „Experimentalsysteme“ (Matzke 2015, 191) verstanden, in denen sich individuelle künstlerische Fragen mit institutionellen Bedingungen des Theaters überkreuzen, in denen Arbeitsszenarien inszeniert werden und spezifische Praktiken zum Einsatz kommen (vgl. ebd., 191f.). Die Tätigkeit des Probens wird aktuell in Verbindung mit einem künstlerischen Forschungsbegriff gebracht, den es aus sprechwissenschaftlicher Sicht zu betrachten lohnt.

Der Artikel beleuchtet zwei Perspektiven der Probenforschung: Zunächst werden forschungsmethodische Aspekte dargelegt und Theaterproben mit ihren Spezifika als wissenschaftlicher Untersuchungsgegenstand betrachtet. Anschließend wird ein Blick auf die (sprech)künstlerische Probenarbeit als künstlerisch-forschende Praxis geworfen und damit das Verständnis von Sprechkunst erweitert. 


\section{Probenprozessforschung (Anna Wessel)}

\subsection{Methodologische Aspekte}

Theaterproben sind transitorisch und sind, wenn sie Forschungsgegenstand werden, „strenggenommen bereits Geschichte“ (Steinbeck 1981, 179). Weil sie erst durch die an den Proben Beteiligten hergestellt werden, sind Theaterproben mit ihren spezifischen Praktiken in ihrer Form, im Ablauf und bzgl. ihrer Funktionen analysierbar (vgl. Roselt 2017, 3). Probenprozessuntersuchungen können damit Einblicke geben, „wie im dialogischen Zwischengeschehen etwas entsteht oder hervorgerufen wird“ (ebd. 4f.). Dafür haben sich qualitativ-interdisziplinäre Ansätze wie bspw. praxeologische Forschungsansätze (vgl. dazu u.a. Husel 2014; Otto 2014, 141) und Arbeiten mit einem ethnomethodologischen Paradigma als gewinnbringend erwiesen. Sie schließen sich gegenseitig nicht aus, sondern gehen von dem gemeinsamen Grundverständnis aus, die (Proben)Praxis aus sich heraus zu erforschen (vgl. Wessel 2017).

\subsubsection{Beobachten und Dokumentieren}

In der Theaterwissenschaft, der Linguistik und der Sprechwissenschaft werden ethnografische Datenerhebungen mittels teilnehmender Beobachtung durchgeführt. Mit einem ethnografischen Vorgehen ist eine Offenheit gegenüber dem Forschungsfeld verbunden, welches wiederum erst durch das Forschungsinteresse definierbar wird. Zugleich sind die konkreten Forschungsfragen erst im Feld aufspürbar, was bedeutet, dass die „Feldbildung und Perspektivfindung [...] in ihrer konstruktiven Wechseldynamik entscheidende Bestandteile des probenethnografischen Forschungsprozesses [darstellen, AW], sie konstituieren sich gegenseitig." (Quick 2020, 45). Die teilnehmende Beobachtung verläuft dabei offen, unstrukturiert und reflexiv (zu methodischen Aspekten der Probenbegleitung s. Wessel 2017) im stetigen Wechsel von aktiv- und passiv teilnehmend, denn:

"The demarcation between insider and outsider is very strong in the theatre, and never more so than when it is a matter of rehearsals, for 
many directors fear the disruptive impact an outsider can have on the chemistry that is occuring in the room." (McAuley 2012, S. 6f.)

Weil ethnografische Beschreibungen durch Wahrnehmungsselektionen und durch die Interpretationen der Forscher*innen gekennzeichnet sind (vgl. u. a. Deppermann et al. 2016, 16; Krämer 2014, 104), wird dafür plädiert die Probenprozesse, wenn möglich, nicht nur schriftlich in Text- und Feldtagebüchern, sondern auch mit Audio- und Videoaufnahmen zu dokumentieren. Diese Medien fokussieren zwar nur ausgewählte Aspekte des Probengeschehens (vgl. u.a. Wartemann 2011, 247; Quick 2020, 48; McAuley 1998, 81), stellen aber in den entstandenen Aufnahmen zusätzliches Material zu ethnografischen Beschreibungen dar und eröffnen damit weiterführende Analysemöglichkeiten, wie bspw. ethnografische Videoanalysen oder multimodale Gesprächsanalysen (vgl. u.a. Tuma et al. 2013, 36; Deppermann 2000, 2008). Zusätzlich bieten sie die Grundlage für ein befremdetes und kritisches Bewusstsein während der Analysen (vgl. McAuley 1998, 76).

\subsubsection{Sichten und Sortieren}

Schon im Feld, während der Datenerhebung, werden die Daten (schriftliche Daten, Audio- und Videomaterial) organisiert. Für die Analysen werden sie anschließend aufbereitet. Das geschieht, indem die Audio- und Videodaten verschriftet und die in den Feldnotizen vermerkten Beschreibungen gesichtet und systematisiert werden. In einem nächsten Schritt können die Daten mehrstufig induktiv kodiert werden. Kodieren stellt im Rahmen von Inhaltsanalysen den analytischen Kern dar, kann aber auch analytische Vorstufe zu sich anschließenden Feinanalysen sein. Um große Datenmengen, wie sie in Probenprozessuntersuchungen entstehen, für die Datenorganisation und Datenanalyse aufzuarbeiten, sind qualitative Analyseproramme wie MAXQDA hilfreich. Für das Sampling werden spezifische Fallkollektionen gebildet und transkribiert und die Audio- und Videodaten werden aufbereitet. Aus diesen vorbereitenden Schritten bildet sich neues Material (bspw. überarbeitete ethnografische Beschreibungen, Transkripte, Videozusammenstellungen), das die Grundlage für die sich anschließenden Analysen bildet. 


\subsubsection{Analysieren}

Analysevorgehensweisen sind in theaterwissenschaftlichen Probenprozessforschungen nur rudimentär beschrieben. Auf der Arbeitskonferenz „Methodologische Diskurse der aktuellen Probenforschung“, auf der 2019 Probenforscher*innen aus der Theaterwissenschaft, der Linguistik und der Sprechwissenschaft über methodische Herangehensweisen diskutierten, zeigte sich, dass bisherige Forschungen interdisziplinär gearbeitet und hauptsächlich ethnografische Herangehensweisen, qualitative Inhaltsanalysen und Verfahrensweisen der Grounded Theory angewendet haben (vgl. Quick 2020, 55).

Auch in der sprechwissenschaftlichen Probenprozessforschung werden ein interdisziplinärer Zugang verfolgt und die Kombination verschiedener Analysezugänge erprobt. Die Analysemethoden sind gegenstandsabhängig. Für die Untersuchung performativer Texterarbeitungsverfahren kombinierte Kiesler (2019, 93ff.) qualitativ-analytische Kodierverfahren mit aufführungs- und inszenierungsanalytischen Ansätzen und auditiven Analysen. Für die Untersuchung interaktiver Aspekte der künstlerischen Probenarbeit (Wessel i. Vorb.) werden induktive Kodierverfahren mit multimodalen Gesprächsanalysen und prozessorientierten Videoanalysen gekoppelt, um interaktive Aspekte künstlerischer Prozesse sowohl mit mikroskopischem als auch mit einem makroskopischen Zugriff analysieren zu können. Dabei ist ein iteratives, daten- und methodentriangulierendes Vorgehen ebenso grundlegend wie die Intersubjektivierung durch Datensitzungen im Expertenkreis.

\subsubsection{Mikroskopischer Zugriff auf Proben}

Das Vor- und Nachspielen ist eine zentrale Vermittlungspraktik in der Theaterarbeit, die auch aus der sprechkünstlerischen Textarbeit bekannt ist. Mit multimodalen Gesprächsanalysen (vgl. u.a. Deppermann 2008; speziell für Probenprozesse: Schmidt et al. 2020; Wessel 2020) kann sie in ihrer künstlerischen und interaktiven Dimension analysiert werden. Die sequentielle Analyse der Videomitschnitte und ihrer multimodalen Transkripte ermöglicht eine mikroskopische Betrachtung, wie Schauspieler*innen und das Regieteam die Probenwirklichkeit herstellen. Damit sind Betrachtungen bzgl. der Dialogizität, der (künstlerischen) Formen und zu verschiedenen Funktionen möglich. So konnte auf diesem Weg herausgearbeitet werden, dass durch das Vorspielen 
Unsagbares sagbar wird (vgl. Wessel 2020). Es wird sowohl retrospektiv zum Erinnern oder Korrigieren bereits erarbeiteter Fassungen vorgespielt, als auch prospektiv zur Entwicklung neuer szenischer Formen. Bezüglich der künstlerischen Dimensionen lassen sich sowohl aspektualisierende als auch holistische Spielformen ausmachen, die für das Anzeigen von nicht gewünschten Formen (aspektualisierend) bzw. für Wunsch-Varianten (holistisches Spiel) eingesetzt werden. Auf organisatorischer Ebene lässt sich feststellen, dass das Vorspielen immer in Kombination mit verbalen Rahmungen realisiert wird (vgl. Wessel 2020). Multimodale Analysen können sehr kleinteilig verschiedene Dimensionen einer Probenpraktik zugänglich machen und vor allem bzgl. der Struktur und des Einsatzes wertvolle mikroskopisch-prozessuale Erkenntnisse liefern.

\subsubsection{Makroskopischer Zugriff auf Probenprozesse}

Um Szenenerarbeitungen nachzeichnen zu können, wird mit einem makroskopischen Verständnis auf die Daten geblickt. Mit ethnografischen Videoanalysen ist es möglich die großen Zeiträume von bis zu siebenwöchigen Probenprozessen nicht nur auf Grundlage ethnografischer Beschreibungen untersuchen zu können, sondern auch sie anhand von Videomaterial global und prozessorientiert zu analysieren. Die Nachzeichnung einer Szenenentwicklung kann unter verschiedenen (interaktiven, ästhetischen, methodisch-didaktischen, etc.) Gesichtspunkten vorgenommen werden (vgl. McAuley 1998, 81). Dafür wird zunächst eine spezifische Szene aus dem Stück ausgewählt und aus dem Videomaterial wird eine entsprechende Fallkollektion erstellt. Anschließend können durch fokussierte Videoanalysen und Analysen der Verschriftungen und der ethnografischen Notizen dieser Szenenerarbeitungen ausgewählte prozessuale Aspekte des Probens analysiert werden.

So eigen und individuell künstlerische Prozesse sind, so spezifisch und gegenstandbezogen sind auch die methodischen Wege sie zu beforschen. Die vorgestellten Methoden stellen einen möglichen Weg von Probenprozessforschungen dar. Dabei wird die Prozessualität sowohl inhaltlich als auch methodisch berücksichtigt und dadurch werden die in der Probenprozessforschung bisher noch nicht kombinierten Konzepte von mikroskopischen und makroskopischen Zugriffen erprobt. Wie anhand des mikroskopischen Zugriffs 
auf Proben skizziert wurde, bilden u.a. Praktiken des Probierens einen Forschungsgegenstand der Probenprozessforschung.

\subsection{Probenpraktiken als wissenschaftlicher Untersuchungsgegenstand}

Praktiken des Probierens sind vielgestaltig und betreffen verschiedene Aspekte der Probenarbeit. In theaterwissenschaftlichen Untersuchungen werden unterschiedliche Praktiken beschrieben. Grundlegend sind u.a. Konzipieren und Interpretieren, auch das Improvisieren und Arrangieren sowie Auswendiglernen und Üben bilden den Kern des Probierens (vgl. Matzke 2014, 270). Aber auch das Unterbrechen von Spieleinheiten, das Treffen von Entscheidungen und das Wiederholen sind charakteristische Merkmale der Probenarbeit (vgl. Roselt 2017, 6). In der Texterarbeitung kommt außerdem die Arbeit mit Vereinfachungen und Übertreibungen zum Tragen. Probenkonstituierend ist die "Transformation ,natürlicher Verhaltensweisen' in ,komponierte Sequenzen"“ (Schechner 1990, 152f.). Diese unterschiedlichen Praktiken werden durch die Probenteilnehmer*innen initiiert, koordiniert und strukturiert und stellen „ästhetische, politische, soziale und ökonomische Dimensionen künstlerischer Praxis“ dar (Matzke 2014, 270). Begreift man Proben als eine kollektive, performative und künstlerische Praxis, so lassen sich die darin enthaltenen Praktiken des Probierens auf verschiedenen Ebenen einordnen und untersuchen. Globalere Praktiken sind beispielsweise das Konzipieren oder Arrangieren. Praktiken wie das Unterbrechen oder Übertreiben betreffen spezifischere Probehandlungen. Die Praktik des Vor- und Nachspielens (vgl. Hinz 2011b) kann, wie in Kap. 2.1. skizziert wurde, sowohl aus ästhetischem Interesse als auch unter methodisch-didaktischen Fragestellungen reflektiert werden.

Die Untersuchung von Probenpraktiken kann Arbeitsweisen und Anforderungen der Probenarbeit in der zeitgenössischen Theaterpraxis aufdecken. Damit können Impulse für die Weiterentwicklung der (sprech)künstlerischen bzw. schauspielerischen Arbeit gesetzt werden und die Anwendung künstlerischer Verfahren, Methoden und Herangehensweisen kann innerhalb von Ausbildungsprozessen reflektiert und erweitert werden. Durch Kooperationen mit verschiedenen Theatern im deutschsprachigen Raum waren Forschungsarbeiten zur Beobachtung und Untersuchung der Probenpraxis möglich. Doch 
Theaterproben bilden nicht nur einen wissenschaftlichen Untersuchungsgegenstand, sie lassen sich vielmehr selbst als Instrument einer künstlerischen Forschungstätigkeit verstehen.

\section{Sprechkünstlerische Forschungspraxis (Julia Kiesler)}

\subsection{Die Probe als Instrument künstlerischer Forschung}

Untersuchungen im Bereich der sprechkünstlerischen Kommunikation stellen sich im Sinne einer anwendungsorientierten Forschung einerseits als eine Forschung über oder für die Kunst dar, andererseits existiert die sprechkünstlerische Praxis als „das bewusst gestaltete, gesprochene künstlerische Wort in unterschiedlichen Kommunikationssituationen für ein Publikum (bzw. für einen oder mehrere Hörer)“ (Haase 2013, 177). Stand lange Zeit das sprechkünstlerische Werk (sei es in Form eines Gedichtvortrags, eines Hörbuchs oder einer Theateraufführung) sowie dessen Wirkung und Wirkungsbedingungen im Mittelpunkt der wissenschaftlichen Betrachtung, hat man in jüngerer Zeit im Rahmen der Probenprozessforschung damit begonnen, ebenso Arbeitsweisen und künstlerische Praktiken wissenschaftlich zu untersuchen (vgl. z. B. Kiesler 2019). Wenig Beachtung fand dabei bisher im sprechwissenschaftlichen Kontext jedoch, dass die Probe selbst Wissen generiert und damit als Instrument künstlerischer Forschung begriffen werden kann. Was Matzke für die Probe im Theater formuliert, gilt auch für die Probenarbeit im sprechkünstlerischen Kontext, zu der theatrale Proben- und Kommunikationsformen u.a. gehören.

„In den Proben wird nicht nur eine Aufführung vorbereitet, werden nicht nur Abläufe eingeübt, in der Probenarbeit wird auch ein spezifisches Wissen generiert. Als Prozess der Wissensgenerierung trifft sich im Begriff des Probens das Theater mit der Wissenschaft." (Matzke 2012, 19)

In diesem Sinne lässt sich das Proben als forschende Tätigkeit in der Kunst verstehen, die eigene Methoden und Verfahrensweisen entwickelt, zum Ein- 
satz bringt und Erkenntnisse erzielt (zu der von Henk Borgdorff eingeführten Unterscheidung in eine Forschung über die Künste, für die Künste und in den Künsten vgl. Jung 2016, 25).

\begin{abstract}
„Probend werden unterschiedliche Formen des Wissens hervorgebracht, angewendet und transformiert, die in Körperpraktiken, Arbeitstechniken und ästhetischen Verfahren zum Ausdruck kommen. Spezifisch für das Proben ist dabei die Verschaltung praktischer und theoriegeleiteter Prozesse. Wissensformen, die an den jeweiligen Akteur und dessen Körper gebunden sind, treffen auf Konzepte und Texte." (Matzke 2015, 190)
\end{abstract}

Die an Akteur*innen und deren Körper gebundenen Wissensformen, von denen Matzke hier spricht, können als implizites Wissen oder als Embodied Knowledge bezeichnet werden, zwei Begriffe, mit denen die sogenannte Künstlerische Forschung operiert. Sie bezeichnen das in Handlungskompetenzen eingelagerte Wissen und ermöglichen „Weisen der erfahrenden Welterschließung [...], die in begrifflich repräsentierter Form nicht zu fassen sind“ (Dubach/Badura 2015, 123).

\title{
3.2 Exkurs: Zum Begriff der „Künstlerischen Forschung“
}

Künstlerische Forschung unterscheidet sich von allgemeiner Kunst und von wissenschaftlicher Forschung. Der künstlerischen Forschung geht es um einen „dezidierten Umgang mit einem klar benennbaren und bestimmten Wissensbestandteil, der künstlerisch reflektiert wird“ (Caduff 2010, 116). Dabei eröffnet insbesondere die Übertragung von Wissen aus nicht künstlerischen Bereichen in künstlerische Kontexte neue Bedeutungsebenen. Die Anfänge der künstlerischen Forschung liegen in den 1990er Jahren. Sie ist hauptsächlich aus dem Bereich der Visual Art entstanden und steht im europäischen Raum im Zusammenhang mit der Akademisierung der Kunststudiengänge im Zuge der Bologna-Reform. Ihr Forschungsbegriff ist umstritten und verweigert sich bis heute einer einheitlichen Bestimmung. 
Hauptsächlich wird künstlerische Forschung an den Begriff der Aisthesis, der sinnlichen Wahrnehmung gebunden. Sie strebt nach sinnlicher Erkenntnis und findet dort ihre Berechtigung, „wo sie intervenierend und intermittierend in Wissenschaftsdiskurse wie auch Alltagswelten eingreift, um diese weiterzuentwickeln, zu verwandeln oder zu verschieben und in überraschende, manchmal auch unverständliche Richtungen voranzutreiben" (Henke et al. 2020, 18). Künstlerische Forschung zeichnet sich durch eine eigene Weise des Denkens, durch „ästhetisches Denken“ aus, das dem philosophischen und wissenschaftlichen Gedanken, „seiner Explikation durch die Sprache“, wie Henke et al. $(2020,32)$ betonen, in nichts nachsteht. Es verwendet „nur andere mediale Formen und Weisen von Ausdrücklichkeit“ (ebd.). Das Ergebnis künstlerischer Forschung muss nicht notwendigerweise in schriftlicher Form dokumentiert werden. Es kann sich z. B. in Form einer Ausstellung, einer Aufführung, eines Videos oder auch in Form eines sprechkünstlerischen Ereignisses präsentieren. Kennzeichnend für eine forschende künstlerische Praxis ist das Wechselverhältnis von künstlerischem Handeln und Prozessen der Reflexion. Sie bringt Kunst und Wissenschaft zusammen und fördert ein kollektives Arbeiten zwischen den Disziplinen (vgl. Ziemer 2015, S. 171).

Die Verortung sprechkünstlerischen Handelns im Feld der künstlerischen Forschung soll hier in Zusammenhang mit der Praxis des Probens gebracht werden, die sich - wie Matzke es für Theaterproben formuliert - „im Spannungsfeld von Wiederholung und forschendem Suchen im Unbekannten“ bewegt (Matzke 2012, 20). Laut Matzke $(2015,189)$ verweist der Begriff der Probe etymologisch auf das Bedeutungsfeld des Versuchs und wird speziell in der jüngeren Theatergeschichte in Verbindung mit den Begriffen Experiment oder Labor gebracht, die eine forschende Praxis implizieren.

Es stellt sich an dieser Stelle die Frage, wie in der theater- bzw. sprechkünstlerischen Probenarbeit Wissen generiert wird und wie dieses Wissen vermittelt werden kann. Welche Erkenntnisse lassen sich durch das (sprech)künstlerische Gestalten eines Textes sowie durch das Anwenden bestimmter Proben- und Herstellungspraktiken gewinnen? Es wird im Folgenden ein Blick auf ausgewählte Probenpraktiken geworfen, die sich als Strategien einer künstlerischen Forschung bestimmen lassen, insofern sie selbst Wissen hervorbringen. 


\subsection{Künstlerisch-forschende Probenpraktiken}

Durch das Sprechen von künstlerischen Texten bzw. durch deren künstlerische Erarbeitung lassen sich Erkenntnisse gewinnen. Hierfür müssen jedoch praktisches Tun und Reflektieren in einen stetigen Austausch treten, „der die Voraussetzung für den Schritt über sich selbst hinaus in das mitteilbare Bewusstsein darstellt“ (Bästlein/Gloor 2015, 212). Erst wenn Schauspieler*innen oder Sprechkünstler*innen durch die künstlerische Tätigkeit neu gewonnene Erkenntnisse intersubjektiv erfahrbar machen, kann von Sprechkunst als künstlerischer Forschung gesprochen werden. Aus diesem Grund ist es wichtig, Herangehensweisen und Praktiken der künstlerischen Arbeit mit dem Text zu reflektieren, auch weil sich der Umgang mit Texten und gesprochener Sprache im zeitgenössischen Theater verändert hat. Es unterliegen nicht nur theatrale Darstellungsformen oder sprechkünstlerische Erscheinungsweisen einem ständigen Wandel, sondern auch deren Herstellung innerhalb von Probenprozessen, in denen verschiedenste Probenpraktiken und Arbeitsweisen zum Einsatz kommen bzw. gefunden werden.

In ihrem Handbuch zur Künstlerischen Forschung führen Badura et al. (2015) eine Reihe von künstlerischen Forschungspraktiken auf, die wir auch aus der Theaterarbeit bzw. aus der sprechkünstlerischen Textarbeit kennen. Hierzu gehören u.a. Praktiken wie recherchieren, denken und reflektieren, improvisieren, experimentieren, formieren und arrangieren, inszenieren, interagieren, proben, kollektives arbeiten oder komponieren (vgl. Badura et al. 2015, $111 \mathrm{ff}$.). Wie sich speziell sprechkünstlerisches Handeln in derartigen Praktiken als künstlerisches Forschen manifestieren kann, wird im Folgenden anhand einer Auswahl skizziert.

\subsubsection{Recherchieren}

Das Recherchieren als künstlerisch-forschende Praktik hat im zeitgenössischen Theater zunehmend an Interesse gewonnen, geht es hier nicht immer um die Umsetzung eines Stücks, sondern oftmals um Stückentwicklungen oder projektartige Arbeiten, in denen verschiedenste Texte und Stoffe zugunsten eines Themas miteinander in Verbindung gebracht werden. Texte werden überhaupt erst generiert oder als Material behandelt, das bearbeitet wird und in das sich 
Regieteams und Schauspieler*innen als Co-Autor*innen einschreiben. Wissen wird im Prozess der Recherche neu zugänglich gemacht und kombiniert (vgl. Orlow 2015, 201). Beispielsweise wird durch das Führen von Interviews mit sogenannten Experten des Alltags (Rimini Protokoll) neues Wissen sowie Textmaterial für eine Produktion gewonnen und mit Texten anderer Quellen kombiniert oder in ein bestehendes Stück integriert. Weiterhin kann sich Recherchieren im theatralen bzw. sprechkünstlerischen Kontext im lesenden Erarbeiten eines Themas, in der Beschäftigung mit dem Autor und der Entstehungszeit eines literarischen Textes oder in einem formal- oder materialbezogenen Sinn im Sichten von Video- und Audiomaterial wiederspiegeln. Das Anschauen von Filmen oder das Hinzuziehen von Videoclips dient als Recherche dem Finden von Spiel- und Sprechweisen. Auch hierin kennzeichnet sich eine Probenpraxis als Forschungsprozess, dessen Ziel nicht allein eine Inszenierung ist, „sondern eine Selbst-Reflexion der theatralen Praxis selbst“ (Matzke 2015, 190).

Aber auch körperliches oder stimmliches Agieren kann als Recherche zur Generierung von körperlichem, vokalperformativem oder szenischem Material genutzt werden. Zum Beispiel führte die Analyse ausgewählter Ausschnitte von Theateraufführungen und Vokalperformances sowie das stimmliche Erproben bestimmter vokalperformativer Techniken zur eigenständigen Entwicklung von sprecherisch-stimmlichen Ausdrucksmöglichkeiten, mit denen die Schauspielstudierenden im Rahmen des Labors „Potentiale des Musikalischen im sprachlichen Kontext" an der Hochschule der Künste Bern Texte erarbeiteten. Im Zentrum dieses Labors stand die Frage, wie der Inhalt eines Textes nicht nur dargestellt, sondern erfahrbar gemacht werden kann. Es wurden Möglichkeiten eines musikalischen Umgangs mit dem Text sowie die Stimme als sinnlich-materielles Phänomen erforscht. Heutzutage ist die Frage, wie etwas dargestellt werden kann, konkret mit der Frage verbunden, „wie, wo, mit wem und unter welchen Bedingungen etwas recherchiert wird“ (Orlow 2015, 204). Dies gilt insbesondere für das zeitgenössische Theater und dessen Probenpraxis. 


\subsubsection{Lesen und Hören auf der Probe}

Durch das mehrmalige Lesen und Sprechen eines Textes, durch das Schmecken der Worte, durch das Assoziieren von Eindrücken und sinnlichen Wahrnehmungen, die sich hinter den Worten verbergen, durch das sprechende Eintauchen in den Rhythmus lernen wir einen Text zu begreifen, ihn nicht nur analytisch, sondern auch sinnlich zu verstehen. Dieser Vorgang begegnet uns im Erlesen oder Ersprechen eines Textes, einem bekannten ersten methodischen Schritt der sprechkünstlerischen bzw. gestischen Erarbeitung eines Textes. Das laute Lesen kann innerhalb der Probenarbeit aber auch zu Erkenntnissen in Bezug auf die Auswahl, Reduktion und Personenzuschreibung von Textmaterial für eine erst während des Probenprozesses entstehende Textfassung führen. So wurden beispielsweise im Rahmen der Produktion Faust. Der Tragödie Erster Teil (Goethe) in der Regie von Claudia Bauer am Konzerttheater Bern Entscheidungen, welche Textstellen gestrichen werden, welche gesprochen oder als Regieanweisung für das szenische Spiel bzw. als Improvisationsmaterial genutzt werden, über das mehrfache Lesen des Textmaterials auf insgesamt sieben Leseproben zu Beginn des Probenprozesses getroffen (vgl. Kiesler 2019, 156f.). Das Ziel dieses Vorgehens war es, zu einer „spielbaren Fassung“ zu gelangen und herauszufinden, welche Erzählweise man für jede Szene findet (ebd., 157).

Auch in der Probenarbeit des Regisseurs Volker Lösch und des Chorleiters Bernd Freytag spielt das laute Lesen und Hören von Texten für den Auswahl- und darüber hinaus für den Kompositionsprozess der Sprechchöre eine wichtige Rolle. Aus dem Leseprozess der für die Produktion Biedermann und die Brandstifter (Max Frisch) am Theater Basel gewonnenen Interviewtexte von Menschen mit einem Migrationshintergrund wurden Erkenntnisse für die chorische Textarbeit gewonnen.

„Textpassagen werden einzeln, zu zweit, in kleineren Gruppen, von allen, nur von den Frauen oder nur von den Männern gelesen. Das gemeinsame Lesen erfüllt verschiedene Funktionen. Zum einen geht es darum, dass alle Beteiligten das zur Verfügung stehende Textmaterial und damit die aus den Interviews der Einzelnen gewonnenen Geschichten kennenlernen, zum anderen möchte die Regie mögliche 
Konstellationen ausprobieren und die Stimmen der einzelnen Männer und Frauen hören. Volker Lösch und Bernd Freytag suchen nach Gestaltungs-, Aufteilungs- und Sprechmöglichkeiten für das generierte Textmaterial, auch wird getestet, was sowohl quantitativ als auch qualitativ mit der Laiengruppe realisierbar ist.“ (Kiesler 2019, 154f.)

Eine Textzuschreibung zu einzelnen oder mehreren Personen musste hier erst gefunden werden und war nicht bereits durch die Figurenangabe eines Stückes vorgegeben. Die Probe wurde zum Test für die Frage Wer spricht?, die u.a. durch das Sprechen und Hören der Texte geklärt wurde.

\subsubsection{Improvisieren und Üben}

Die Improvisation ist uns als künstlerische Tätigkeit bestens aus den Aufführungskünsten Musik, Theater, Tanz und Performance bekannt. „Als Improvisieren wird der Umgang mit unvorhergesehenen Situationen bezeichnet, wo Plan und Aufführung einer Handlung zusammenfallen, aber auch die besondere Fähigkeit, auf unerwartete Ereignisse adaptiv und möglichst adäquat zu reagieren." (Bertinetto 2015, 147). Bertinetto $(2015,148 \mathrm{ff}$.) unterscheidet zwischen dem Improvisieren als spezifischer Praktik des Entdeckens und der Improvisation als künstlerischem Verfahren, innerhalb dessen spezifische Forschungsinhalte verfolgt werden können, letzteres v.a. um die Auseinandersetzung von Künstlerinnen mit Materialien, Situationen, Formen und Interaktionen auszuloten.

In der Theaterarbeit werden in der Improvisation entwickelte Vorgänge oftmals geübt. „Das Improvisieren und Üben wird zu einer Aufgabe, Material zu produzieren, das dann in der weiteren Probenarbeit bearbeitet, neu zusammengesetzt, montiert wird [...].“ (Matzke 2012, 232). Als Beispiel sei an dieser Stelle das bereits angesprochene Labor „Potentiale des Musikalischen im sprachlichen Kontext" genannt, das Julia Kiesler an der Hochschule der Künste Bern mit der Vokalperformerin Franziska Baumann im Juni 2020 im Studienbereich Theater durchführte. Die Studierenden bekamen Aufgabenstellungen, in denen sich Improvisieren und Wiederholen, Erfinden und Üben überlagerten. Es wurde u.a. mit dem Text Lenz von Georg Büchner gearbeitet. Im Zentrum stand die Frage, wie wir den seelischen Zustand von 
Lenz musikalisch erfahrbar machen können (zum Begriffsverständnis einer musikalischen Arbeit am Text vgl. Kiesler 2019, 242 ff). Wir suchten zunächst im Text nach Schlüsselwörtern, die uns einen Hinweis geben und entdeckten die Polarität Lenzens Wahrnehmung in Wortpaaren wie „Gipfel - Täler“, „,aufwärts - abwärts“, „verschlang - enthüllte“, „suchen - nichts finden“, „nahe - fern“, „fern verhallend - gewaltig heranbrausten“, „Himmel - Erde“ etc. (vgl. Büchner 1973, 85f.). Büchners Novelle drückt den inneren Zustand des Schriftstellers Lenz zu Beginn des Textes in Naturbildern aus. Die Naturgewalten und deren Beschreibung aus der spezifischen Wahrnehmung von Lenz dienen als Metapher für seine schwer verbalisierbare Stimmung. Unser Versuch lag darin, das „Nicht-Sagbare“ seines Unruhezustands auf einer musikalischen Ebene sinnlich zu erfahren und erfahrbar werden zu lassen. Die Studierenden erhielten die Aufgabe, zu ausgewählten Wörtern, Formulierungen, Satzfragmenten oder Sätzen Klangmaterial zu entwickeln und damit in der Kleingruppe zu improvisieren. Dieses Klangmaterial konnte ausschließlich stimmliche/vokalperformative Verlautbarungen oder auch Textmaterial beinhalten, mit dem sie experimentieren durften, z. B. indem sie einzelne Wörter oder Sätze dekonstruierten, wiederholten, überlagerten etc. In Übungen wurden verschiedene Musikalisierungsstrategien und vokalperformative Stimmtechniken erarbeitet, wie z. B. die Arbeit mit Tonhöhen-, Lautstärke- oder Tempowechseln, mit stimmlichen Dissonanzen oder mit Techniken wie inhale-exhale, Knarzen oder dem Pfeiftonregister. Diese wurden für die Improvisation genutzt und mit dem Inhalt des Textes in Verbindung gebracht. Aus den Improvisationen generierten die Studierenden vokalperformatives Material, das sie für die weitere Erarbeitung des Textes verwendeten. Dabei ging es weniger um die Vertonung des Textes als vielmehr um ein Ausloten des Inhalts und um die sinnliche Erschließung einer seelischen Figurenlage.

\subsubsection{Montieren/Formieren/Arrangieren}

Diese Praktiken der künstlerischen Forschung sind mit der sprechkünstlerischen Tätigkeit in Verbindung zu bringen, insofern sie auf Prozesse des Herstellens von Situationen verweisen, in denen Vorgefundenes neu zueinander in Beziehung gesetzt wird (vgl. Siegmund 2015, 139). Jeder Text kann in eine neue Sprechsituation gestellt werden, aus der sich vielfältige Bezüge und Kontexte 
und damit Interpretationen ergeben. „So sind die künstlerischen Handlungen des Formierens/Arrangierens nicht allein als ein Agieren aufzufassen, sondern auch als ein Reagieren unter Einflüssen eines vorkünstlerisch gebildeten subjektiven Erfahrungsraumes.“ (ebd., 139f.) Es findet ein Transformationsprozess statt, aus dem etwas Neues entsteht.

Auch intertextuelle Arbeitsweisen lassen sich in diesem Zusammenhang beschreiben, die beispielsweise innerhalb eines theatralen Probenprozesses eine Stückvorlage dekonstruieren und mit Texten aus anderen Quellen ergänzen. So werden z. B. in vielen Produktionen des Regisseurs Volker Lösch zusätzliche Texte durch Interviews, durch Diskussionen, durch das Erfinden von Geschichten oder durch Aufgabenstellungen generiert. Diese zusätzlich generierten Texte werden während der Probenarbeit in einem aufwendigen Prozess kodiert, kreativ aufgebrochen und neu verschaltet, indem sie mit anderen Texten oder in eine Stückvorlage montiert werden. Durch das Montageprinzip entsteht eine Textfassung mit einer intertextuellen Struktur, die vielstimmige Diskurselemente freisetzt und verschiedene Perspektiven eines Themas zutage fördert. Hier geht es nicht mehr um die Umsetzung eines Theaterstücks, sondern um das Hervorbringen neuer, sich überlagernder, multiperspektivischer Realitätsebenen (vgl. hierzu: Kiesler 2019, 145ff.).

Die Liste an Praktiken der künstlerischen Probenforschung ließe sich fortsetzen. Beispielsweise wäre auch das chorische Sprechen als eine Form des gemeinschaftlichen Handelns interessant zu betrachten, nicht nur in Bezug auf dessen Erscheinungsform, sondern als gesellschaftliches Modell, das in der chorischen Arbeit erfahren werden kann.

\section{$4 \quad$ Fazit}

Sprechkunst kennzeichnet sich durch ihren performativen Charakter, sie ereignet sich im Vollzug des Sprechens u.a. auf der Bühne. Dabei zielt die sprechkünstlerische Erarbeitung eines Textes oder eines Themas nicht nur auf ein Sprechkunstereignis im Sinne einer Aufführung, sondern ist bestimmt durch den Einsatz bestimmter Praktiken, mit denen der Frage nachgegangen wird, 
wie ein Text gesprochen bzw. ein Thema sprechkünstlerisch gestaltet werden kann.

Wie gezeigt werden konnte, lassen sich diese Praktiken auf unterschiedliche Wege erforschen. Zum einen durch Probenprozessbeobachtungen und deren Analysen in einem anwendungsorientierten, wissenschaftlichen Kontext. Zum anderen durch eine forschende künstlerische Praxis. Denn die Praktiken lassen sich insofern als sprechkünstlerisch-forschende Probenpraktiken definieren, als dass sie Wissen hervorbringen oder neu verschalten. Ihre Reflexion, Aufarbeitung und Didaktisierung innerhalb der Sprechwissenschaft soll an dieser Stelle angeregt werden. Es braucht einerseits eine (sprech)künstlerische Forschungspraxis, die durch die Probenarbeit und damit verbundene Praktiken traditionelle Texterarbeitungsmodelle erweitert und Ansätze entwickelt, mit denen beispielsweise postdramatische Texte erarbeitet werden können. Andererseits braucht es die wissenschaftliche und methodische Reflexion sowie Aufarbeitung von Probenpraktiken und künstlerischen Verfahren im Rahmen der Probenprozessforschung, um sie für die Sprecherziehung und Schauspielausbildung zugänglich zu machen.

\section{Literatur}

Badura, Jens/Dubach, Selma/Haarmann, Anke/Mersch, Dieter/Rey, Anton/Schenker, Christoph/Pérez, Germán Toro (Hg.) (2015): Künstlerische Forschung. Ein Handbuch. Zürich, Berlin: Diaphanes. S. 147-150.

Bästlein, Ulf/Gloor, Valentin (2015): Singen. In: Badura, Jens/Dubach, Selma/Haarmann, Anke/Mersch, Dieter/Rey, Anton/Schenker, Christoph/Pérez, Germán Toro (Hg.): Künstlerische Forschung. Ein Handbuch. Zürich, Berlin: Diaphanes. S. 211-213.

Bertinetto, Alessandro (2015): Improvisieren. In: Badura, Jens/Dubach, Selma/Haarmann, Anke/Mersch, Diete/Rey, Anton/Schenker, Christoph/Pérez, Germán Toro (Hg.): Künstlerische Forschung. Ein Handbuch. Zürich, Berlin: Diaphanes. S. $147-150$.

Büchner, Georg (1973): „Lenz“. In: Büchner, Georg: Dichtungen. Leipzig: Reclam jun. S. 85-108.

Caduff, Corina (2010): Literatur und künstlerische Forschung. In: Caduff, Corina/Siegenthaler, Fiona/Wälchli, Than (Hg.): Kunst und künstlerische Forschung. 
Zürcher Jahrbuch der Künste 2009. Band 6. Zürich: Scheidegger \& Spiess. S. 108-117.

Deppermann, Arnulf (2000): Ethnographische Gesprächsanalyse: Zu Nutzen und Notwendigkeit von Ethnographie für die Konversationsanalyse. In: Gesprächsforschung - Online-Zeitschrift zur verbalen Interaktion (1), S. 96-124.

Deppermann, Arnulf (2008): Gespräche analysieren. Eine Einführung. Wiesbaden: VS Verlag für Sozialwissenschaften. (Qualitative Sozialforschung 3)

Deppermann, Arnulf/Feilke, Helmuth/Linke, Angelika (2016): Sprachliche und kommunikative Praktiken: Eine Annäherung aus linguistischer Sicht. In:

Deppermann, Arnulf/Feilke, Helmuth/Linke, Angelika (Hg.): Sprachliche und kommunikative Praktiken. Berlin, Boston: De Gruyter. S. 1-23. (Jahrbuch des Instituts für Deutsche Sprache, 2015)

Dubach, Selma/Badura, Jens (2015): Denken /Reflektieren (im Medium der Kunst). In: Badura, Jens/Dubach, Selma/Haarmann, Anke/Mersch, Dieter/Rey, Anton/Schenker, Christoph/Pérez, Germán Toro (Hg.): Künstlerische Forschung. Ein Handbuch. Zürich, Berlin: Diaphanes. S. 123-126.

Haase, Martina (2013): Definition und Gegenstand der Sprechkunst. In: Bose, Ines/Hirschfeld, Ursula/Neuber, Baldur/Stock, Eberhard: Einführung in die Sprechwissenschaft. Phonetik, Rhetorik, Sprechkunst. Tübingen: Narr Francke Attempto Verlag. S. 177-181.

Henke, Silvia/Mersch, Dieter/Strässle, Thomas/van der Meulen, Nicolaj/Wiesel, Jörg (Hg.) (2020): Manifest der künstlerischen Forschung. Eine Verteidigung gegen ihre Verfechter. Zürich: Diaphanes.

Hinz, Melanie/Roselt, Jens/Merz, Gunther (Hg.) (2011a): Chaos und Konzept. Proben und Probieren im Theater. Berlin: Alexander Verlag.

Hinz, Melanie (2011b): Vorspiel und Nachahmung auf Probe. In: Hinz, Melanie/Roselt, Jens/Merz, Gunther (Hg.): Chaos und Konzept. Proben und Probieren im Theater. Berlin: Alexander Verlag, S. 72-96.

Husel, Stefanie (2014): Grenzwerte im Spiel. Die Aufführungspraxis der britischen Kompanie »Forced Entertainment«; eine Ethnografie. Bielefeld: Transcript (Theater 65).

Jung, Eva-Maria (2016): Die Kunst des Wissens und das Wissen der Kunst. Zum epistemischen Status der künstlerischen Forschung. In: Siegmund, Judith: Wie verändert sich Kunst, wenn man sie als Forschung versteht? Bielefeld: Transcript Verlag, S. 23-43.

Kiesler, Julia (2016): „Jede Szene ist ein neues Glück.“ Verfahren der Texterarbeitung innerhalb des Probenprozesses Faust (J.W. Goethe) in der Regie von Claudia Bauer am Konzerttheater Bern. In: Unger, Angela (Hg.): Sprechen in unterschiedlichen Kontexten: Radio, Wirtschaft, Theater, Fremdsprachenunterricht. Beiträge zum 2. Doktorandentag der Halleschen Sprechwissenschaft. Reflexionen des Gesellschaftlichen in Sprache und Literatur - Hallesche Beiträge Band 5. Hg. v. Antos, Gerd/Bremer, Thomas/Hirschfeld, Ursula/Jäger, Andrea/Nell, Wer- 
ner/Richter, Angela. Online-Publikation. S. 51-72. http://digital.bibliothek. uni-halle.de/pe/content/titleinfo/2422792 (Letzter Zugriff: 15.08.2020)

Kiesler, Julia (2017): Methodische Aspekte einer musikalischen Arbeit am Text - Ein Probenbeispiel. In: Hannken-Illjes, Kati/Gauß, Eva Maria/Könitz, Friederike/Franz, Katja/Marx, Silke (Hg.): Stimme - Medien - Sprechkunst. Sprache und Sprechen. Band 49. Baltmannsweiler: Schneider Verlag Hohengehren. S. 62-73.

Kiesler, Julia (2019): Der performative Umgang mit dem Text. Ansätze sprechkünstlerischer Probenarbeit im zeitgenössischen Theater. Berlin: Verlag Theater der Zeit. (Recherchen 149). https://www.theaterderzeit.de/buch/der_performative_ umgang_mit_dem_text/ (Letzter Zugriff: 03.09.2020)

Kiesler, Julia/Petermann, Claudia (2019): Zwischen Virtuosität und Persönlichkeit. Perspektiven für eine gegenwärtige Schauspielausbildung. In: Kiesler, Julia/Petermann, Claudia (Hg.): Praktiken des Sprechens im zeitgenössischen Theater. Berlin: Theater der Zeit. S. 10-40. (Recherchen 141).

Krämer, Hannes (2014): Die Praxis der Kreativität. Eine Ethnografie kreativer Arbeit. Bielefeld: Transcript (Gesellschaft der Unterschiede, 18).

Matzke, Annemarie (2012): Arbeit am Theater. Eine Diskursgeschichte der Probe. Bielefeld: Transcript. (Theater 48).

Matzke, Annemarie (2014): Probe. In: Fischer-Lichte, Erika/Kolesch, Doris/Warstat, Matthias (Hg.): Metzler-Lexikon Theatertheorie. Stuttgart: Metzler, S. 270-273.

Matzke, Annemarie (2015): Proben. In: Badura, Jens/Dubach, Selma/Haarmann, Anke/Mersch, Dieter/Rey, Anton/Schenker, Christoph/Pérez, Germán Toro (Hg.): Künstlerische Forschung. Ein Handbuch. Zürich, Berlin: Diaphanes. S. 189-192.

McAuley, Gay (1998): Towards an Ethnography of Rehearsal. In: NTQ 14 (53), S. 75-85.

Milde, Andrea (2019): Linguistics in drama processes. In: Urban Language \& Literacies 2019, 2019 (WP 251), S. 1-19.

Orlow, Uriel (2015): Recherchieren. In: Badura, Jens/Dubach, Selma/Haarmann, Anke/Mersch, Dieter/Rey, Anton/Schenker, Christoph/Pérez, Germán Toro (Hg.) (2015): Künstlerische Forschung. Ein Handbuch. Zürich, Berlin: Diaphanes. S. 201-204.

Otto, Ulf (2014): Doing Theatre. Theater, Wissenschaft und Praxis. In: Baumbach, Gerda/Darian, Veronika/Heeg, Günther/Primavesi, Patrick/Rekatzky, Ingo (Hg.): Momentaufnahme Theaterwissenschaft. Leipziger Vorlesungen. Berlin: Theater der Zeit. S. 137-146. (Recherchen 117).

Quick, Tamara Yasmin (2020): Methodologische Diskurse der aktuellen Probenforschung - Zwischenbericht aus einer (noch jungen) Forschungsdisziplin in der Theaterwissenschaft. In: Forum Modernes Theater. 31 /1-2 (2020). Tübingen: Gunter Narr Verlag. S. 39-63.

Rastetter, Claudia (2017a): Das Playback-Verfahren in der Arbeit der Regisseurin Susanne Kennedy. In: Hannken-Illjes, Kati/Gauß, Eva Maria/Könitz, Friederi- 
ke/Franz, Katja/Marx, Silke (Hg.): Stimme - Medien - Sprechkunst. Sprache und Sprechen. Band 49. Baltmannsweiler: Schneider Verlag Hohengehren. S. 49-61. Rastetter, Claudia (2017b): Jelinek sprechen - Textarbeit im Probenprozess zu Elfriede Jelineks Wut in der Regie von Nicolas Stemann. In: Wagner, Roland (Hg.): Sprechen. Zeitschrift für Sprechwissenschaft, Sprechpädagogik, Sprechtherapie, Sprechkunst. Heft 64. Heidelberg, S. 68-81.

Roselt, Jens (2017): Probe als Aufführung. http://www.theaterwrede.de/ flausenkongress/wp-content/uploads/sites/3/2017/04/Jens-Roselt_Regietischals-Metapher.pdf (Letzter Zugriff: 27.08.2020)

Schechner, Richard (1990): Theater-Anthropologie. Spiel und Ritual im Kulturvergleich. Reinbek bei Hamburg: Rowohlt (Rowohlts Enzyklopädie Kulturen \& Ideen, 439).

Schmidt, Axel/Krug, Maximilian/Messner, Monika/Wessel, Anna (Hg.) (2020) (in Vorb.): Instruktionen in Theaterproben. In: Gesprächsforschung - Online-Zeitschrift zur verbalen Interaktion.

Siegmund, Judith (2015): Formieren /Arrangieren. In: Badura, Jens/Dubach, Selma/Haarmann, Anke/Mersch, Dieter/Rey, Anton/Schenker, Christoph/Pérez, Germán Toro (Hg.) (2015): Künstlerische Forschung. Ein Handbuch. Zürich, Berlin: Diaphanes. S. 139-142.

Steinbeck, Dietrich (1981): Probleme der Dokumentation von Theaterkunstwerken. In: Helmar Klier (Hg.): Theaterwissenschaft im deutschsprachigen Raum. Texte zum Selbstverständnis. Darmstadt: Wissenschaftliche Buchgesellschaft, S. 179-191. (Wege der Forschung, 548).

Tuma, René; Schnettler, Bernt; Knoblauch, Hubert (2013): Videographie. Einführung in die interpretative Videoanalyse sozialer Situationen. Wiesbaden: Springer Fachmedien (Qualitative Sozialforschung).

Wartemann, Geesche (2011): Experimentierfelder. Eine Kameraethnographische Studie zum Modell des Helios Theater, das Publikum im Probenprozess zu beteiligen. In: Hinz, Melanie/Roselt, Jens/Merz, Gunther (Hg.): Chaos und Konzept. Proben und Probieren im Theater. Berlin: Alexander Verlag, S. 242-259.

Wessel, Anna (2016): „Sprache ist eine Waffe. Wenn sie nichts auslöst, kann man sie auch weglassen. Handlungsstrategien und sprechkünstlerische Realsierungen in Probenprozessen zeitgenössischer deutschsprachiger Inszenierungen“. In: Unger, Angela (Hg.): Sprechen in unterschiedlichen Kontexten: Radio, Wirtschaft, Theater, Fremdsprachenunterricht. Beiträge zum 2. Doktorandentag der Halleschen Sprechwissenschaft. Reflexionen des Gesellschaftlichen in Sprache und Literatur - Hallesche Beiträge Band 5. Hg. v. Antos, Gerd/Bremer, Thomas/Hirschfeld, Ursula/Jäger, Andrea/Nell, Werner/Richter, Angela. Online-Publikation. S. 73-90. http://digital.bibliothek.uni-halle.de/pe/content/titleinfo/2422792 (Letzter Zugriff: 15.08.2020) 
Wessel, Anna (2017): Methodische Aspekte sprechwissenschaftlicher Probenprozessforschung. In: Hannken-Illjes, Kati/Franz, Katja/Gauß, Eva-Maria/Könitz, Friederike/Marx, Silke (Hg.): Stimme - Medien - Sprechkunst. Sprache und Sprechen. Band 49. Baltmannsweiler: Schneider Verlag Hohengehren GmbH. S. 74-85.

Wessel, Anna (in Vorb.) (2020): Vorspielen - instruktive Praktik in der Theaterprobe. In: Schmidt, Axel/Krug, Maximilian/Messner, Monika/Wessel, Anna (Hg.) (in Vorb.): Instruktionen in Theaterproben. In: Gesprächsforschung - Online-Zeitschrift zur verbalen Interaktion.

Wessel, Anna (in Vorb.): »watch your language«. Praktiken des Probierens und Interkation in Theaterproben. (Arbeitstitel). (Diss.-schrift)

Ziemer, Gesa (2015): Kollektives Arbeiten. In: Badura, Jens/Dubach, Selma/Haarmann, Anke/Mersch, Dieter/Rey, Anton/Schenker, Christoph/Pérez, Germán Toro (Hg.): Künstlerische Forschung. Ein Handbuch. Zürich, Berlin: Diaphanes. S. 169-172. 



\section{Die Sprechbuehne und das Kunstmuseum Moritzburg Halle (Saale) - Geschichte einer Kooperation}

Zusammenfassung: Die Sprechbuehne wurde 1992 im Rahmen des damaligen Diplom-Studienganges Sprechwissenschaft an der Martin-Luther-Universität Halle-Wittenberg gegründet. Szenische Lesungen und Collagen sind ein Markenzeichen ihrer Produktionen. Mit Studierenden im Bachelor- und Master-Studiengang Sprechwissenschaft werden die Inszenierungen professionell erarbeitet und in der Öffentlichkeit präsentiert. Die Kooperation mit dem Kunstmuseum Moritzburg Halle (Saale) hat dabei eine herausragende Stellung. Für das Museum werden speziell für aktuelle Kunstausstellungen konzipierte Projekte entwickelt und im Museum aufgeführt. Künstlerisches Ziel dieser Zusammenarbeit ist die sinnliche Erlebbarkeit und die Verbindung verschiedener Künste. Einen Höhepunkt des Zusammenwirkens von Sprechbuehne und Kunstmuseum stellte das Einar-Schleef-Projekt (2019) „Ich bin ein anderer in mir, den muß ich fragen“ anlässlich des 75. Geburtstages des Künstlers dar.

Stichwort: Sprechbuehne, Universität Halle, Kunstmuseum Moritzburg Halle (Saale), Einar Schleef

Abstract: "Sprechbuehne" was founded in 1992 as part of the diploma course in Speech Science at the Martin Luther University Halle-Wittenberg. A trademark of their productions are staged readings and collages. The professional developed productions are presented to the public with students of the bachelor's and master's degree courses in Speech Science. The cooperation with Moritzburg Art Museum Halle (Saale) has an outstanding position. Projects specially conceived for current art exhibitions are developed for the museum and performed in the museum. The artistic goal of this collaboration is the sensorial experience and the connection of different arts. The Einar Schleef project (2019) „I am someone else in myself, whom I have to ask“ on the occa- 
sion of the artist's 75th birthday represented a highlight in the collaboration between the "Sprechbuehne" and the Art Museum.

Keywords: SprechBuehne, University of Halle, Moritzburg Art Museum Halle (Saale), Einar Schleef

\section{„Was Kunst werden soll, muss brennen.“ Einar Schleef}

\section{Die Sprechbuehne (Martina Haase)}

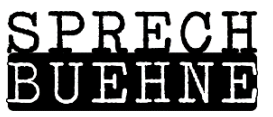

Die Sprechbuehne wurde 1992 im Rahmen des damaligen Diplom-Studienganges Sprechwissenschaft an der Martin-Luther-Universität Halle-Wittenberg von Martina Haase (Dozentin an der Abteilung Sprechwissenschaft und langjährige Sprecherzieherin an verschiedenen Theatern) gegründet und wird seitdem von ihr künstlerisch geleitet. Szenische Lesungen und Collagen sind das Markenzeichen der Sprechbuehne.

Ausgewählte Texte, die i. d. R. keine Bühnentexte im Sinne des dramatischen Genres sind, werden dramaturgisch mit Hilfe einer szenischen Grundidee als Textcollage entwickelt und mittels Sprache, Stimme, Bewegung, Interaktion und Spiel für die Bühne umgesetzt, unterstützt durch Kostüme, Maske, Bühnenbild und Musik. Die professionelle Realisierung der Inszenierungen erfolgt mit Studierenden im Bachelor- und Master-Studiengang Sprechwissenschaft. Die öffentlichen Aufführungen finden v.a. im Kunstmuseum Moritzburg Halle (Saale) und im Puppentheater Halle statt. Für das Museum sind das eigens für aktuelle Kunstausstellungen konzipierte Produktionen. Jede Inszenierung steht ein bis drei Jahren auf dem Spielplan. In der Regel werden 5 bis 20 Vorstellungen pro Produktion gespielt.

Das Kunstmuseum Moritzburg Halle (Saale) ist aus dem 1885 gegründeten Städtischen Museum für Kunst und Kunstgewerbe der Stadt Halle hervorgegangen und wird seit 2014 von Thomas Bauer-Friedrich als Direktor geleitet. 
Die Kooperation besteht seit Gründung der Sprechbuehne. Bis 2019 wurden über 15 gemeinsame Projekte realisiert.

Neben Auftragswerken für das Kunstmuseum werden auch Projekte mit anderen Kooperationspartnern verwirklicht, z. B. mit der Stiftung Luther-Gedenkstätten in Sachsen-Anhalt oder mit den Franckeschen Stiftungen Halle, und natürlich mit der halleschen Universität. Darüber hinaus gibt es freie Produktionen, die im Selbstauftrag entwickelt werden. Auf diese Weise sind in den knapp 30 Jahren des Bestehens der Sprechbuehne über 50 Inszenierungen entstanden. Gastspiele führten die Sprechbuehne in verschiedene Städte, u.a. nach Berlin, Leipzig, Münster, in die Schweiz nach Luzern, ans Staatstheater Kassel, auf das Theaterschiff in Potsdam, ins Novalis-Museum Schloss Oberwiederstedt, ins Schuhmann-Haus Leipzig. Höhepunkte in jüngerer Zeit waren mit der Inszenierung (2013-2016) In Verjandlungen mit Ernst - Ein Spiel mit Texten von Ernst Jandl die Teilnahme am Internationalen Theaterfestival „Drehbühne Brno“ in Tschechien und ein Gastspiel im Rahmen des klangTUNNEL MUSIKFESTes in Erpel bei Bonn.

Unterstützt wird die Arbeit der Sprechbuehne durch ein Netzwerk von verschiedenen professionellen Künstlerinnen und Künstlern im Bereich Kostüm und Maskenbild (Claudia Franke-Hildebrandt), Musik, Bühnen- und Lichttechnik, Grafik und Design (Prof. Frithjof Meinel), Videokunst (Conny Klar), Fotografie (Markus Scholz), die seit Jahrzehnten uneigennützig unsere Arbeit begleiten.

Die Sprechbuehne und damit die hallesche Sprechwissenschaft wirkt durch diese künstlerischen Aktivitäten über die Universität hinaus in die Stadtgesellschaft und ist inzwischen eine feste Größe im öffentlichen kulturellen Leben von Halle mit einem treuen Publikum geworden.

\section{$1.1 \quad$ Gründung der Sprechbuehne}

Im Lehrangebot der halleschen Universität wurde in den späten 1930er Jahren eine Lehrveranstaltung Feiergestaltung im Institut für Sprechkunde angeboten. Das war der Vorläufer des Seminars Programmgestaltung im Curriculum des Diplom-Studienganges Sprechwissenschaft in den 1960er Jahren. So erlebte die Erstautorin es noch als Studentin und so übernahm sie die Leitung die- 
ser Lehrveranstaltung als junge Assistentin. Damalige Praxis war es, dass die Studierenden zu vorgegebenen Themen literarische-musikalische Programme entwickelten, die im Seminar dann vorgestellt und ggf. öffentlich präsentiert wurden. Vorherrschendes Paradigma der sprechwissenschaftlichen Lehre auf dem Gebiet der Vortragskunst (damalige Bezeichnung) war die Verwendung der Rezitation als dominierendes sprecherisches Stilmittel in Abgrenzung zu theatralen Mitteln und Formen, also eine Beschränkung der künstlerischen Ausdruckmittel allein auf das gesprochene Wort.

Die literarischen Vorlagen waren vornehmlich Lyrik und Prosa. Aus dieser Situation heraus und als Reflektion auf die künstlerische Praxis entstand die Idee, aus der eher uniinternen „Laborsituation“ einer Lehrveranstaltung verstärkt in die Kunstpraxis und damit in die Öffentlichkeit zu gehen. Der Fokus der Lehrveranstaltung wurde dabei in Richtung szenische Arbeit und theatrale Präsentation verschoben. Damit eröffnete sich für die Studierenden die Möglichkeit, ein Stück Theaterbetrieb in allen Phasen einer künstlerischen Produktion zu durchlaufen: von der Konzeption des Stückes (die Spielvorlage wird von Sprechbuehne selbst erarbeitet) über Leseproben, Bühnenproben bis zur Premiere. Sie lernen die an solchen Produktionen beteiligten Gewerke (wie Kostüm, Maske, Bühnenbild, Lichttechnik, Musik, Video, Fotografie), aber auch das notwendige Management und die Öffentlichkeits- und PR-Arbeit näher kennen. Die Kreativität der Student*innen wird herausgefordert und in professionelle Bahnen gelenkt. Sie können ihre eigenen Fähigkeiten in der sprechkünstlerisch-szenischen Umsetzung von Literatur ausloten und erwerben Bühnenerfahrung als nachhaltiges Erlebnis. Gleichzeitig ermöglicht es den Studierenden eine besondere Teamerfahrung und trägt zu ihrer Persönlichkeitsentwicklung bei. Die Arbeit im Kontext der Sprechbuehne bildet damit einen künstlerisch-kreativen Bestandteil des Studiums Sprechwissenschaft. Sie ermöglicht die Verbindung von Theorie, Methodik und künstlerischer Praxis im Lehrgebiet Sprechkunst, fordert und fördert damit die studentische Qualifikation und bereitet auf das Teilgebiet Sprecherziehung an künstlerischen Hochschulen als ein zukünftiges Berufsfeld vor. Seit 1993 ist diese Form der Lehrveranstaltung (als Sprechkünstlerische bzw. Szenische Projektarbeit) und die damit verbundene Arbeitsweise fester, wahlobligatorischer Bestandteil 
der Studien- und Prüfungsordnung des Studienganges Sprechwissenschaft in Halle.

\subsection{Charakteristika der Kooperation mit dem Kunstmuseum Moritzburg Halle (Saale)}

Künstlerisches Ziel dieser Zusammenarbeit ist die sinnliche Erlebbarkeit und die Verbindung verschiedener Künste: der bildendenden Kunst und der Sprechkunst. Die Sprechbuehne begleitet seit rund 20 Jahren ausgewählte Ausstellungen des Kunstmuseums mit szenischen Lesungen und Collagen. Diese werden themenbezogen speziell für eine bestimmte Ausstellung erarbeitet. Bereits im ersten Jahr der Existenz der Sprechbuehne wurde die Produktion Wanderer zwischen den Welten. Drei Projekte zum literarischen Expressionismus im Rahmen der Ausstellung Grafik des deutschen Expressionismus realisiert. Ein offizieller Kooperationsvertrag existiert seit 2009.

Die Produktionen sind thematisch ausstellungsbegleitend und finden in verschiedenen Räumlichkeiten des Museums in Korrespondenz mit den Bildern und Objekten statt. Gemälde, Grafiken und Objekte bilden das Bühnenbild. Das Besondere für die Zuschauer*innen: sie sitzen inmitten der Ausstellung und erleben so hautnah die Korrespondenz von Bild und Wort - Bild wird Wort und Szene. Es entsteht eine einmalige Wirkung, die die Museumsarbeit synästhetisch unterstützt - eine Wirkung, die so z. B. im Theater nicht zu erreichen ist. Die bildende Kunst im Raum beeinflusst auch das gesprochene Wort und regt die Mitwirkenden bei der Umsetzung der Texte an. Die Kooperation wird dadurch gewinnbringend für die Zuschauer*innen und damit für die Öffentlichkeit, für das jeweilige Team der Sprechbuehne und für das Museum.

\subsection{Rahmenbedingungen}

Basis für die Projekte ist eine enge Zusammenarbeit mit Kunstwissenschaftlerinnen und besonders mit den jeweiligen Kurator*innen der Ausstellung. Neben der Bereitstellung von kunstwissenschaftlicher Literatur bieten die Mitarbeiter*innen des Museums für das Sprechbuehnen-Team Vorträge und Führungen an. All das sind Grundlagen für die Konzeptionsentwicklung der 
geplanten Produktion. Die Studierenden erhalten Einblicke in verschiedene Epochen der bildenden Kunst, in die jeweilige Zeit- und Kunstgeschichte und zu einzelnen Künstler*innen. Die Entwicklung der Konzeption und die Inszenierungsarbeit erfolgen eigenständig und eigenverantwortlich im Team der Sprechbuehne. Die dramaturgischen Konzepte werden zwar in Korrespondenz mit der jeweiligen Ausstellungskonzeption entwickelt, sie sind aber keine Verbalisierung der Ausstellungsinhalte, sondern folgen selbstgewählten Aussageabsichten.

Nicht unerwähnt bleiben darf an dieser Stelle der Bereich der Infrastruktur, der vom Museum personell und materiell zur Verfügung gestellt wird, beginnend bei Vorankündigungen, Programmdruck, Kartenverkauf und Werbung. Erforderlich sind Probenmöglichkeiten außerhalb der Öffnungszeiten vor Ort, weil die Räume des Museums eine besondere, für das Sprechen schwierige Akustik haben. Bühnenbau und Beleuchtung müssen realisiert werden. Eine weitere Besonderheit ist das Bühnenbild, was in der Regel Bestandteil der jeweiligen Ausstellung ist. Ggf. werden auch eigens für die Vorstellung Gemälde gehängt. Ein Museum ist kein Theater, der logistische und arbeitstechnische Aufwand für die Realisierung solcher Projekte ist beträchtlich! Allen Beteiligten des Museums sei an dieser Stelle herzlich gedankt für ihr Engagement!

\section{$1.4 \quad$ Grundlagen der Textcollagen}

Am Anfang steht der Ausstellungsplan des Kunstmuseums, in der Regel 1-2 Jahre im Voraus. Soviel Vorlaufzeit wird auch benötigt, um eine Inszenierung von der Konzeption über das Erstellen des Textkonvoluts und die eigentlichen Bühnenproben bis zur Premiere zu bringen.

Die Entscheidung für ein Projekt der Sprechbuehne zu einer spezifischen Ausstellung wird begleitet von entsprechenden Überlegungen und Vorstellungen des Museums. Sie sind abhängig vom Ausstellungskonzept und dessen thematischen Schwerpunkten sowie von dem geplanten Begleitprogramm. Daraus folgen erste Ideen der Sprechbuehne, die dann eigenständig weiterentwickelt werden.

Von größter Relevanz sind in dieser Phase die Recherchen zu geeigneter Primär- und Sekundärliteratur, z. B. Briefwechsel oder Selbstzeugnisse der 
Künstler*innen, also Literatur, die für die Bühnenumsetzung geeignet erscheint. Im Ergebnis besteht die Textgrundlage der Inszenierungen aus einem Mix von literarischen und nichtliterarischen Texten bis hin zu Archivmaterial wie Verträgen oder Rechnungen. So wurde z. B. beim Feininger-Projekt (2001/ 2016) der Schriftverkehr zwischen dem Künstler und dem Magistrat der Stadt Halle verwendet. Typisch für die meisten Sprechbuehnen-Produktionen ist der Collagecharakter der Spielvorlage, also das Montieren von verschiedenen Texten und Textteilen. Beim Feininger-Projekt bildeten der Briefwechsel von 1929 bis 1931 zwischen Lyonel Feininger und seiner Frau Julia sowie der zwischen Feininger und Alois Schardt, dem damaligen Direktor des Kunstmuseums Moritzburg Halle (Saale) die Basis, ergänzt durch zahlreiche Zeitdokumente. Feininger schuf in diesem Zeitraum im Auftrag der Stadt Halle seine berühmten elf Stadtporträts. Für die Produktion Komm, Trost der Nacht, o Nachtigall. Literatur, Sprache und Musik im 17. Jahrhundert (2014) bildeten Barocklyrik und Zeitdokumente um den Fürsten August von Sachsen in seiner Residenzstadt Halle die Textgrundlage. Für dieses Projekt interessierte v. a. das Lebensgefühl zwischen carpe diem und memento mori in der Zeit um den 30-jährigen Krieg und die höfische Festkultur und Sprache. Um diese Schwerpunkte für das Publikum erlebbar zu machen, wurden auch Teile eines 1669 gedruckten und von Mitgliedern des Hofes in Halle uraufgeführten Theaterstückes Eintracht stärckt Heyrath: Oder/ Ballet Der Wolgerathenen Ehe in die Textcollage eingefügt.

Die Textquellen für die Collagen stammen aus unterschiedlichen Epochen: begonnen bei der Renaissance (2015: DENKmal Cranach. Ausstellung Lucas Cranach der Jüngere. Entdeckung eines Meisters. Stiftung Luther-Gedenkstätten in Sachsen-Anhalt) über die Zeit des Barocks, des Expressionismus und der Klassischen Moderne bis in die Gegenwart. Einer der thematischen Schwerpunkte der Produktionen ist dabei die Kunst des Expressionismus und der klassischen Moderne, bedingt durch den Sammlungsschwerpunkt des Museums. Allein elf von zwanzig Produktionen stehen in Bezug zu dieser Kunst und ihren Künstler*innen. Beispiele dafür sind Franz Marc (2010: Mein lieber, lieber blauer Reiter. Franz Marc und Else Lasker-Schüler. Ein Briefwechsel in Bildern) oder Emil Nolde (2013: Noldes Gemälde „Abendmahl“ - ein Kunstkampf oder wie die Moderne ins Museum kam. 


\subsection{Das Einar-Schleef-Projekt 2018/19}

Einen Höhepunkt der Zusammenarbeit von Sprechbuehne und Kunstmuseum stellte das Einar-Schleef-Projekt anlässlich des 75. Geburtstages des Künstlers dar. Einar Schleef (1944-2001), genialischer, vielseitiger Künstler, war eine „schillernde Persönlichkeit, die es sich und anderen gern unbequem machte“ (Behrens 2002, 8) - im eigenen Leben und in seiner Kunst. Seine Theaterinszenierungen waren von Anfang an heiß umstritten in Ost und West. Aber in seiner Kunst, seinen Bildern und seinen Büchern gab es eines nicht: den Kompromiss. Wolfgang Behrens charakterisiert Leben und Wirken dieses Ausnahmekünstlers: „Jugend in der Provinz, vielversprechender Karrierebeginn in der DDR, Flucht und Isolation, Skandale und Erfolge, dutzendweise abgebrochene Theaterprojekte und schließlich ein früher, einsamer Tod."(ebd.).

\section{Zur Dramaturgie und Inszenierung}

Nach einer konzeptionellen Vorarbeit von etwa einem Jahr begann die dramaturgische und szenische Arbeit mit den Studierenden. Sie können sich in verschiedenen Bereichen der Theaterarbeit erproben: als Darsteller*innen, in der Regieassistenz und Soufflage, auf technischem Gebiet (Licht, Ton) und ggf. auf den Gebieten Musik und Komposition. Insgesamt bestand das studentische Team bei diesem Projekt aus 15 Teilnehmer*innen.

Ich bin ein anderer in mir, den muss ich fragen. Einar Schleef. Der Theaterregisseur, Autor und Maler Einar Schleef in seinen Tagebüchern. Der Stücktitel war gleichzeitig Leitmotiv der Produktion, Schleefs Tagebücher lieferten in gewisser Weise das dramaturgische Grundgerüst.

Schleef begann im Alter von neun Jahren bis zu seinem Tod regelmäßig Tagebuch zu schreiben. Von Anfang an bedeuteten sie für ihn ein Mittel der Selbstverständigung. Diese fünf Tagebücher (ein Konvolut über 2500 Seiten), die Schleef in späteren Jahren mit Kommentaren versah und für die Veröffentlichung vorbreitete, waren gleichzeitig Inspiration und Haupt-Textquelle, ergänzt durch weitere Werke des Künstlers wie Droge Faust Parsifal oder den Roman Gertrud, Interviews mit Schleef sowie Theaterkritiken seiner Inszenierungen und Äußerungen von Zeitgenossen. 
Grundidee, Leitgedanke und zugleich Anliegen der Produktion war es, den bild- und wortgewaltigen Künstler und Menschen Schleef einem breiteren Publikum zugänglich zu machen, indem wir Einblicke in sein Leben und Werk ermöglichen. Eine besondere Würdigung erfährt dabei seine Rolle als Erneuerer des Chortheaters. Umgesetzt haben wir diese Grundidee über eine zeitlich-chronologische Anordnung der Texte in Bezug zu wesentlichen Lebens- und Arbeitsstationen des Künstlers. Ein Liveticker zu Beginn jeder neuen Szene gibt eine kurze Orientierung für die Zuschauer*innen:

LIVETICKER: 1964-76 Ostberlin: Studium Malerei und Bühnenbild an der Kunsthochschule Berlin-Weißensee / Beginn der Theaterarbeit als Bühnenbildner, später Regisseur (2. Bild)

Eigens komponierte Sprechchöre (von einer ehemaligen Studentin) aus Schleef-Zitaten wurden als strukturierende Elemente innerhalb der gesamten Inszenierung eingesetzt, was zugleich eine Referenz an Schleef als Erneuerer des Chortheaters ist.

Szenenbilder (Stückfassung @Sprechbuehne Halle 2018)

Prolog Ich bin ein anderer in mir (Sprechchor)

1. Bild Vom Nichtwiederaufschreibenkönnen

Kindheit und Jugend in Sangerhausen 1944-1964

2. Bild Was Kunst werden soll, muss brennen (Sprechchor)

Studium und erste Theaterarbeiten in Ostberlin 1964-1976

3. Bild Wovor bin ich weggelaufen (Sprechchor)

Heimat, Flucht, Isolation 1976-1985

4. Bild Der Chor ist krank (Sprechchor)

Theaterarbeit I 1985-1990

5. Bild 14 Vorhänge (Sprechchor)

Theaterarbeit II 1990-1996

Epilog Ein thüringischer Dionysos 
Das Schleef-Zitat Ich bin ein anderer in mir, den muss ich fragen bot den Rahmen für die Etablierung der szenischen Grundidee und dem Entwickeln von Spielsituationen:

- Schleef spricht mit sich selbst / befragt sich / zweifelt an sich / träumt u. ä. - im „Tagebuch“-Grundgestus

- Schleef spricht mit anderen - Interviewsituation, Telefonat - Andere sprechen über Schleef - Theaterkritiken, Nachrufe

Eine wesentliche Arbeitsweise bei der szenischen Umsetzung der Texte war die Improvisation. Die szenische Etablierung der Texte in die jeweilige Situation innerhalb der einzelnen Szenenbilder wurde durch Bewegungs-Choreografien erreicht - in Anlehnung an Chor-Choreografien.

Das Figuren-Ensemble bestand aus vier Frauen und zwei Männern: die Männer stellen i. W. die Figur Schleef dar. In einzelnen Szenen sind die Schleef-Texte auf das gesamte Ensemble als Schleef-Figur verteilt. Der Liveticker wird von einer Moderatorin-Figur realisiert. Darüber hinaus gibt es Interviewpartnerinnen, Theaterkritiker, Theaterwissenschaftler und Weggefährten.

Die Kostüme wurden in Anlehnung an Chorkleidung entwickelt und unterstreichen die Einheitlichkeit, die für den Sprechchor charakteristisch ist. Eine Besonderheit bilden dabei die einheitlich geschnittenen Oberteile für alle, die eigens mit Motiven nach Schleefs Gemälde Klage (Telefonzelle XV) bedruckt wurden. Dieses Motiv findet sich auch im Bühnenbild. 3 Schleef-Gemälde aus der sog. Serie Telefonzellen bildeten das Bühnenbild.

Die Inszenierung hat bisher neun Vorstellungen erlebt, fünf davon allein im Kunstmuseum Moritzburg Halle (Saale).

Im Rahmen der Kleinen Fächer-Wochen (KFW), initiiert und gefördert durch die Hochschulrektorenkonferenz und das Bundesministerium für Bildung und Forschung, konnte eine DVD für dieses Sprechbuehnen-Projekt produziert werden. Die öffentliche Video-Premiere fand als Abschluss der KFW am 31.01.2020 an der halleschen Universität statt. Eine DVD kann auf Anfrage zur Verfügung gestellt werden. 
Ein großer Dank gebührt der Abteilung Sprechwissenschaft und Phonetik an der Martin-Luther-Universität Halle-Wittenberg für die jahrzehntelange ideelle, strukturelle und finanzielle Zusammenarbeit und Unterstützung der Sprechbuehne.

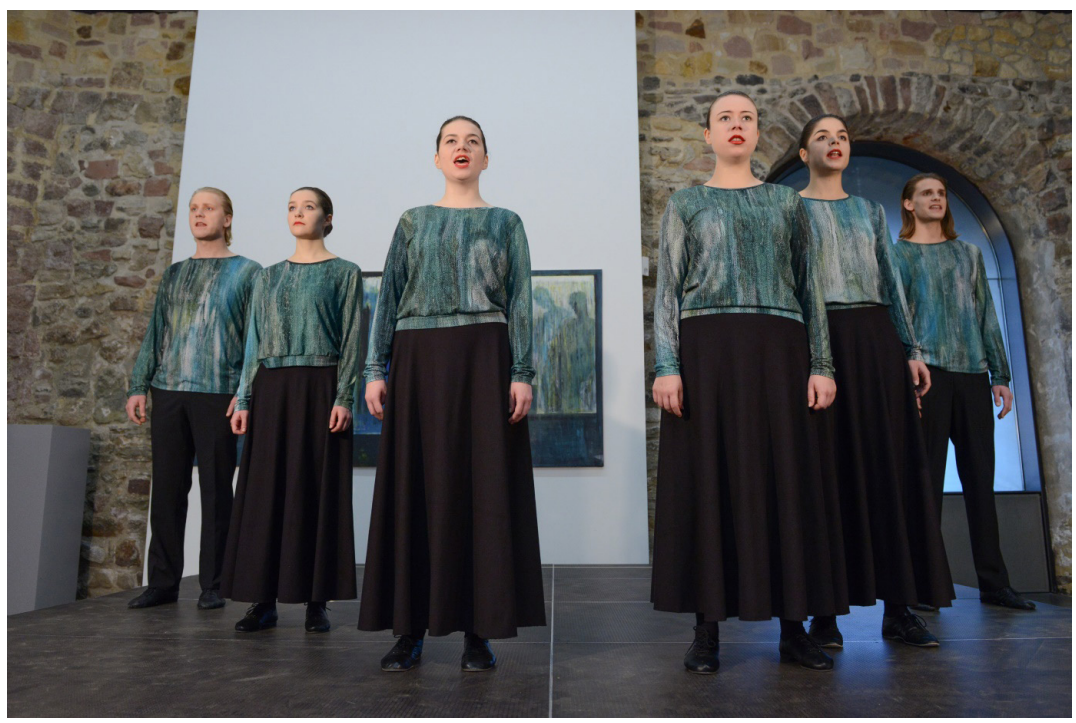

Abb.1: Szenenfoto: Markus Scholz (Bildnachweis Schleef-Gemälde, VG Bild 2018)

\subsection{Auswahl-Szenografie Sprechbuehne - Kunstmuseum Moritzburg Halle (Saale)}

1992: Wanderer zwischen den Welten. Drei Projekte zum literarischen Expressionismus. Lesung und Aktion im Rahmen der Ausstellung Grafik des deutschen Expressionismus

1999: Bedecke deinen Himmel, Zeus. Gedichte und Balladen von Goethe. Lesung zur Ausstellung Barlach und Goethe - eine Ausstellung der Ernst Barlach Gesellschaft Hamburg 
2000: Die Pfade der Abgeschiedenen. Annäherung an Georg Trakl. Lesung. Rahmenprogramm zur Ausstellung Die Welt gebiert eine neue Zeit - Zeichnungen und Aquarelle von 1900 bis 1933

2009: Hoch im Blau sind Orgelklänge... Expressionismus in Schrift und Bild. Literarische Führung

2010: Mein lieber, lieber blauer Reiter. Franz Marc und Else Lasker-Schüler. Ein Briefwechsel in Bildern. Szenische Lesung im Rahmen der Ausstellung Franz Marc und Paul Klee - Dialog in Bildern

2012: In roten Schuhen tanzt die Sonne sich zu Tod. Expressionistische Lyrik. Eine szenische Collage im Rahmen der Ausstellung Wort wird Bild - Illustrationen der „Brücke“-Maler

2013: Die Kunst selbst ist eben meine Sprache. Briefwechsel zwischen Emil Nolde und Max Sauerlandt 1913-1920. Szenische Lesung im Rahmen der Ausstellung Emil Nolde - Farben heiß und heilig

2014: Komm, Trost der Nacht, o Nachtigall. August von Sachsen und die Stadt Halle. Literatur, Sprache und Musik im 17. Jahrhundert. Lesung mit Musik im Rahmen der Ausstellung Im Land der Palme - August von Sachsen, Erzbischof von Magdeburg und Fürst in Halle (1614-1680)

2016: Lyonel Feininger in Halle - wie ein Maler die Stadt wahrnimmt - eine Szenische Collage im Rahmen der Ausstellung Lyonel Feininger - Paris 1912. Die Rückkehr eines verlorenen Gemäldes

2018: Ich bin ein anderer in mir, den muss ich fragen. Einar Schleef. Der Theaterregisseur, Autor und Maler Einar Schleef (1944-2001) in seinen Tagebüchern. Szenische Collage im Rahmen der Ausstellung Ohne Titel [Einar Schleef] + DVD 
Im folgenden Teil beschreibt der Direktor des Kunstmuseums Moritzburg Halle (Saale) aus seiner ganz persönlichen Perspektive die Kooperation zwischen dem Museum und der Sprechbuehne der Universität Halle.

\section{Vom Zusammenführen der Künste. Die Sprechbuehne aus der Sicht des Museums (Thomas Bauer-Friedrich)}

Auch wenn ich von den mehr als 25 Jahren der Existenz der Sprechbuehne mit etwa einem Viertel der Jahre nur einen vergleichsweise kurzen Zeitraum begleiten konnte, möchte ich aus dieser Erfahrung heraus würdigen und anerkennen, was Frau Dr. Haase gemeinsam mit meinen Mitarbeiterinnen und Mitarbeitern unter meinen beiden Vorgängern im Amt des Direktors und in jüngster Vergangenheit gemeinsam mit mir aufgebaut und etabliert hat. Die Produktionen der Sprechbuehne sind eine Bereicherung des Angebots meines Hauses für unser Publikum!

Die Zusammenarbeit mit nicht-musealen kulturellen Partnern aus dem Bereich der „benachbarten“ Künste, der darstellenden Kunst ebenso wie der Musik, ist mir in der Programmgestaltung sehr wichtig und ein explizites Anliegen. Zwar gehören Literatur-, Theater- oder Musikveranstaltungen nicht zu den Kernaufgaben eines Museums, jedoch sind sie für mein Verständnis auch nicht nur dekoratives Beiwerk im Rahmen eines Begleitprogramms. Die Werke der bildenden und angewandten Kunst entstehen nicht im luftleeren Raum, sondern stets angeregt durch eine Vielzahl von Eindrücken und Einflüssen, die die Künstlerinnen und Künstler aufnehmen. Dazu zählt natürlich die Rezeption von literarischen Werken in Form gelesener Texte oder dramatisiert in Form von Inszenierungen auf der Theaterbühne, Konzerte oder Musiktheaterveranstaltungen u. v. m. So war z. B. ein Auslöser für Wassily Kandinskys Weg in die gegenstandslose Kunst im Januar 1911 das Erlebnis eines Konzertes mit Arnold Schönbergs Streichquartett op. 10 von 1907/08 und den drei Klavierstücken op. 11 von 1909. Im Nachgang dieser eindrucksvollen musikalischen Begegnung entstand sein stark abstrahiertes Gemälde Impression III (Konzert). 
In der Vermittlung der Kunst, die wir im Museum präsentieren, ist es mir daher wichtig, dass wir nicht nur die klassischen kunstwissenschaftlichen Vorträge anbieten, in denen Leben und Werk der Künstler vorgestellt und erklärt werden. Mittels Lesungen, Konzerten, kleinen Theateraufführungen oder besonderen szenisch gestalteten Rundgängen durch die Ausstellungen möchte ich unserem Publikum ein Verständnis dafür vermitteln, wie die verschiedenen Künstlerinnen und Künstler zu ein und derselben Zeit aus einer verwandten Geisteshaltung heraus ihre Werke schufen. Im Erlebnis z. B. der Gemälde und szenisch realisierter zeitgenössisch entstandener Texte des Expressionismus ergänzt um Musik der Zeit lässt sich wesentlich besser, quasi am eigenen Leib, erfahren, worum es den Künstlern vor dem Ersten Weltkrieg im Erschaffen ihrer Werke ging. Auf diese Weise stellt sich beim Publikum im Idealfall ein Verstehen ein, mit dem wir auf das Beste jenen altehrwürdigen Bildungsauftrag des Museums erfüllen, dem ich mich als Direktor auch im 21. Jahrhundert verpflichtet fühle.

In diesem Zusammenhang nimmt die Kooperation mit der Sprechbuehne eine besondere Rolle ein, denn die Produktionen, die in den letzten Jahren im Kunstmuseum Moritzburg Halle (Saale) ihre Premiere erlebten, sind nicht einfach Textcollagen zu einem bestimmten Thema, sondern weit mehr als das, es sind eigene Theaterstücke, die Frau Dr. Haase verfasst und mit ihren Studierenden erarbeitet, einstudiert und aufführt. Die Anregung dafür kommt zumeist vom Museum während der Vorbereitung von Sonderausstellungen. Das ist insofern nicht unbedeutend, weil die Sprechbuehne ebenso wie das Museum einen langen zeitlichen Vorlauf benötigt, um die Werke, sei es die Ausstellung oder das Sprechbuehnen-Stück, zu erarbeiten und einer erfolgreichen Premiere entgegenzuführen. Von dieser langen gemeinsamen Vorbereitungszeit profitieren schlussendlich beide: Das Museum kann im Rahmen des Veranstaltungsprogramms zur jeweiligen Sonderausstellung seinem Publikum ein Angebot unterbreiten, das in keinster Weise beliebig oder ersetzbar ist, sondern ganz im Gegenteil genuin mit der Ausstellung und ihrem Gegenstand verbunden ist. Darüber hinaus ist das Ergebnis, die neue Produktion der Sprechbuehne, auch für das Museum immer wieder überraschend und erhellend, wirft sie doch jedes Mal einen verständigen Blick von außen auf den Gegenstand unserer Arbeit. Das Publikum schätzt dies sehr! 
$\mathrm{Zu}$ diesen Besonderheiten auf der inhaltlichen Seite kommen weitere Aspekte, die die Produktionen einmalig machen. So ist jedes Stück speziell auf den Ort im Museum, d. h. in den Räumen der ehemaligen erzbischöflichen Residenz mit dem modernen Museumsneubau von 2008 inszeniert, an dem es aufgeführt werden soll. Ortsbezogene Gegebenheiten müssen berücksichtigt und einbezogen werden, bzgl. der Arbeit der Studierenden vor allem die jeweils unterschiedlichen akustischen Verhältnisse in den Räumen. Darüber hinaus gibt es eine dritte Komponente, die eine Sprechbuehnen-Aufführung für den Rezipienten zu etwas Besonderem macht: die Arbeit mit den originalen Kunstwerken. Wenn möglich bezieht das Bühnenbild einer Sprechbuehnen-Aufführung die ausgestellten Kunstwerke ein oder es werden eigens für die Aufführung Werke aus dem Depot zur Verfügung gestellt. Damit wird das Erlebnis der Aufführung ein dreifach Besonderes: ein eigens für den Anlass und thematischen Kontext einer aktuell laufenden Ausstellung geschaffenes Sprechtheater-Stück, das sich mit dem Inhalt der Ausstellung auseinandersetzt und ein individuell auf den Aufführungsort hin eingerichtetes Stück, das zudem originale Werke der bildenden Kunst aus dem Museum einbezieht.

Neben dem unschätzbaren Wert, den eine so gestaltete Seminararbeit für die Studierenden hat, setzen diese sich automatisch mit Leben und Werk der thematisierten Künstlerinnen und Künstler auseinander, erweitern somit also quasi en passant ihren Bildungshorizont, und erfahren sie darüber hinaus auch die besonderen Herausforderungen einer Sprechtheaterproduktion in einem Kunstmuseum mit seinen ganz eigenen Abläufen und Rahmenbedingungen. Die Studierenden können von einem so ausgerichteten Lehrangebot in jeder Hinsicht nur profitieren.

Einen Höhepunkt in der so gelagerten Zusammenarbeit zwischen der Sprechbuehne und dem Museum war die Einar-Schleef-Produktion im Jahr 2018/19. Anlass war der 75. Geburtstag des 2001 verstorbenen Theaterregisseurs, Schriftstellers und Künstlers und die vor diesem Hintergrund vorbereitete Kabinettpräsentation des Museums Ohne Titel: [Einar Schleef], die den öffentlichkeitswirksamen Abschluss der digitalen Erfassung des bildkünstlerischen Nachlasses Schleefs bildete. Er befindet sich mit mehr als 7100 Arbeiten im Kunstmuseum Moritzburg Halle (Saale). Während die Ausstellung nach drei Monaten Laufzeit im Sommer 2019 vorbei war, ist der Nachlass 
seither dauerhaft unter www.einar-schleef.de recherchierbar. Und anders als die früheren ausstellungsbezogenen Produktionen der Sprechbuehne ist das Schleef-Projekt dank der erfolgten DVD-Produktion ebenfalls zeitlich und örtlich unbegrenzt rezipierbar, was mich insofern besonders freut, weil dieses Stück nicht nur das aufwendigste und vielleicht anspruchsvollste in der Geschichte der Sprechbuehne und mir mit Blick auf den vom Museum verwalteten Nachlass ein besonderes Anliegen war. Im Zusammenklang von Ausstellung und szenischer Aufführung gelang es, dem Publikum die „Naturgewalt“ Einar Schleef und das ihm eigene Kunstverständnis in der bildenden wie in der darstellenden Kunst zu vermitteln. Der Applaus des Publikums bei allen Vorstellungen bewies den Erfolg. In diesem Sinne hoffe ich auf viele neue Kooperationen mit und Aufführungen der Sprechbuehne im Kunstmuseum Moritzburg Halle (Saale) auch in den nächsten Jahren!

\section{Literatur}

Behrens, Wolfgang (2003): Einar Schleef. Werk und Person. Theater der Zeit Berlin. Schleef, Einar (2004-2009): Tagebücher 1953-2001. 5 Bände. Hg. Winfried Menninghaus, Sandra Janßen und Johannes Windrich. Suhrkamp Verlag Frankfurt am Main.

Bildnachweis im Foto: Einar Schleef: Klage (Telefonzelle XV), 1978-83, Mischtechnik auf Leinwand, 150 x $80 \mathrm{~cm}$, Leihgabe aus Privatbesitz, Kulturstiftung Sachsen - Anhalt, Kunstmuseum Moritzburg Halle (Saale) @ VG Bild-Kunst, Bonn 2018. 


\section{Verzeichnis der Autorinnen und Autoren}

Thomas Bauer-Friedrich (Kunstmuseum Moritzburg Halle (Saale)) thomas.b-friedrich@kulturstiftung-st.de

Prof. Dr. Ines Bose (Abt. Sprechwissenschaft und Phonetik, Martin-Luther-Universität Halle-Wittenberg) ines.bose@sprechwiss.uni-halle.de

Dr. Alexandra Ebel (Abt. Sprechwissenschaft und Phonetik, Martin-Luther-Universität Halle-Wittenberg) alexandra.ebel@sprechwiss.uni-halle.de

Dr. Martina Haase (Abt. Sprechwissenschaft und Phonetik, Sprechbuehne, Martin-Luther-Universität Halle-Wittenberg) martina.haase@sprechwiss.uni-halle.de,www.sprechbuehne.uni-halle.de

Prof. Dr. Kati Hannken-Illjes (AG Sprechwissenschaft, Philipps-Universität Marburg) kati.hannkenilljes@uni-marburg.de

Rudolf Hientz (ARD-Aussprachedatenbank, Hessischer Rundfunk) Rudolf.Hientz@hr.de

Prof. Dr. Ursula Hirschfeld (Abt. Sprechwissenschaft und Phonetik, Martin-Luther-Universität Halle-Wittenberg) ursula.hirschfeld@sprechwiss.uni-halle.de

Prof. Dr. Uwe Hollmach (Theaterakademie August Everding und Abt. Sprechwissenschaft und Phonetik, Martin-Luther-Universität Halle-Wittenberg) uwe.hollmach@sprechwiss.uni-halle.de

Dr. Julia Kiesler (Institut Praktiken und Theorien der Künste, Hochschule der Künste Bern) julia.kiesler@hkb.bfh.ch 
Kerstin Kreße (Kinderland Halle gGmbH) kerstin.kresse@vs-sk.de

Franziska Kreutzer (Eigenbetrieb Kindertagesstätten Halle) franziska.kreutzer@halle.de

Michaela Kupietz (Abt. Sprechwissenschaft und Phonetik, Martin-Luther-Universität Halle-Wittenberg) michaela.kupietz@sprechwiss.uni-halle.de

Dr. Stephanie Kurtenbach (Abt. Sprechwissenschaft und Phonetik, Martin-Luther-Universität Halle-Wittenberg) stephanie.kurtenbach@sprechwiss.uni-halle.de

Dr. Friderike Lange (Zentrum für Lehrer*innenbildung, Martin-Luther-Universität Halle-Wittenberg) friderike.lange@zlb.uni-halle.de

Dirk Meyer (Abt. Sprechwissenschaft und Phonetik, Martin-Luther-Universität Halle-Wittenberg) dirk.meyer@sprechwiss.uni-halle.de

Prof. Dr. Baldur Neuber (Abt. Sprechwissenschaft und Phonetik, Martin-Luther-Universität Halle-Wittenberg) baldur.neuber@sprechwiss.uni-halle.de

Dr. Judith Pietschmann (Abt. Sprechwissenschaft und Phonetik, Martin-Luther-Universität Halle-Wittenberg) judith.pietschmann@sprechwiss.uni-halle.de

Kerstin Schuck (Mitarbeiterin der Professur für Sprechwissenschaft, Friedrich-Schiller-Universität Jena) kerstin.schuck@uni-jena.de 
Steffi Schwarzack (Abt. Sprechwissenschaft und Phonetik, Martin-Luther-Universität Halle-Wittenberg) steffi.schwarzack@sprechwiss.uni-halle.de

PD Dr. Robert Skoczek (Abt. Sprechwissenschaft und Phonetik, Martin-Luther-Universität Halle-Wittenberg) robert.skoczek@sprechwiss.uni-halle.de

Prof. Dr. Susanne Voigt-Zimmermann (Abt. Sprechwissenschaft und Phonetik, Martin-Luther-Universität Halle-Wittenberg) susanne.voigt-zimmermann@sprechwiss.uni-halle.de

Prof. Dr. Susanne Vollberg (Abt. Medien- und Kommunikationswissenschaft, Martin-Luther-Universität Halle-Wittenberg) susanne.vollberg@medienkomm.uni-halle.de

Anna Wessel (Abt. Sprechwissenschaft und Phonetik, Martin-Luther-Universität Halle-Wittenberg) anna.wessel@sprechwiss.uni-halle.de

Matthias Winter (freiberuflicher Trainer und Coach für eine angstfreie Unternehmenskultur) kontakt@futureyourculture.de,www.futureyourculture.de 


\section{HALLESCHE SCHRIFTEN ZUR SPRECHWISSENSCHAFT UND PHONETIK}

Die dieser Reihe vorausgehende Reihe „Hallesche Schriften zur Sprechwissenschaft und Phonetik" wurde von Eva-Maria Krech und Eberhard Stock begründet. Die Bände 1-15 wurden von Eva-Maria Krech und Eberhard Stock herausgegeben; die Bände 16-32 von Lutz Christian Anders, Ursula Hirschfeld, Eva-Maria Krech und Eberhard Stock; die Bände 33-51 von Lutz Christian Anders, Ines Bose, Ursula Hirschfeld, Eva-Maria Krech, Baldur Neuber und Eberhard Stock (vgl. http://www.sprechwiss.uni-halle.de/publikationen/).

Bd. 1 Eva-Maria Krech/Eberhard Stock (Hg.) (1996): Beiträge zur deutschen Standardaussprache. ISBN 978-3-7684-6538-0

Bd. 2 Eva-Maria Krech/Eberhard Stock (Hg.) (1997): Sprechen als soziales Handeln. ISBN 978-3-7684-6739-1

Bd. 3 Eva-Maria Krech/Eberhard Stock (Hg.) (1999): Sprechwissenschaft Zu Geschichte und Gegenwart. ISBN 978-3-631-34697-6

Bd. 4 Yvonne Anders (2001): Merkmale der Melodisierung und des Sprechausdrucks ausgewählter Dichtungsinterpretationen im Urteil von Hörern. ISBN 978-3-631-37005-6

Bd. 5 Margret Bräunlich/Baldur Neuber/Beate Rues (Hg.) (2001): Gesprochene Sprache transdisziplinär. ISBN 978-3-631-37730-7

Bd. 6 Eva-Maria Krech (Hg.) (2002): Sprach-, Sprech- und Stimmstörungen interdisziplinäre Kooperation in der Therapie. ISBN 978-3-631-38620-0

Bd. 7 Baldur Neuber (2002): Prosodische Formen in Funktion. ISBN 978-3-631-38998-0

Bd. 8 Eberhard Stock/Ludmila Velickova (2002): Sprechrhythmus im Russischen und Deutschen. ISBN 978-3-631-36631-8

Bd. 9 Ines Bose (2003): dóch da sín ja' nur mûster II. ISBN 978-3-631-50142-9

Bd. 10 Eva-Maria Krech/Eberhard Stock (Hg.) (2003): Gegenstandsauffassung und aktuelle phonetische Forschungen der halleschen Sprechwissenschaft. ISBN 978-3-631-39273-7

Bd. 11 Wieland Kranich (2003): Phonetische Untersuchungen zur Prosodie emotionaler Sprechausdrucksweisen. ISBN 978-3-631-51029-2

Bd. 12 Lutz Christian Anders/Ursula Hirschfeld (Hg.) (2003): Sprechsprachliche Kommunikation. ISBN 978-3-631-50466-6

Bd. 13 Kati Hannken-Illjes (2004): Gute Gründe geben. ISBN 978-3-631-51028-5

Bd. 14 Annaliese Benkwitz (2004): Kontrastive phonetische Untersuchungen zum Rhythmus. ISBN 978-3-631-52902-7

Bd. 15 Norbert Gutenberg (Hg.) (2004): Schreiben und Sprechen von Hörfunknachrichten. ISBN 978-3-631-52009-3

Bd. 16 Christiane Ulbrich (2005): Phonetische Untersuchungen zur Prosodie der Standardvarietäten des Deutschen in der Bundesrepublik Deutschland, in der Schweiz und in Österreich. ISBN 978-3-631-54414-3 


\section{HALLESCHE SCHRIFTEN ZUR SPRECHWISSENSCHAFT UND PHONETIK}

Bd. 17 Christiane Miosga (2006): Habitus der Prosodie. ISBN 978-3-631-54391-7

Bd. 18 Ursula Hirschfeld/Lutz Christian Anders (Hg.) (2006): Probleme und Perspektiven sprechwissenschaftlicher Arbeit. ISBN 978-3-631-54476-1

Bd. 19 Ramona Benkenstein (2007): Vergleich objektiver Verfahren zur Untersuchung der Nasalität im Deutschen. ISBN 978-3-631-56176-8

Bd. 20 Beate Wendt (2007): Analysen emotionaler Prosodie. ISBN 978-3-631-56381-6

Bd. 21 Uwe Hollmach (2007): Untersuchungen zur Kodifizierung der Standardaussprache in Deutschland. ISBN 978-3-631-54261-3

Bd. 22 Ines Bose (Hg.) (2007): Sprechwissenschaft. ISBN 978-3-631-56536-0

Bd. 23 Ute Gonnermann (2007): Quantifizierbare Verfahren zur Bewertung von Dysphonien. ISBN ISBN 978-3-631-57332-7

Bd. 24 Mariam Hartinger (2008): Untersuchungen der Sprechmotorik von Polterern mit Hilfe der Elektromagnetischen Mediosagittalen Artikulographie (EMMA). ISBN 978-3-631-55886-7

Bd. 25 Beate Redecker (2008): Persuasion und Prosodie. ISBN 978-3-631-56929-0

Bd. 26 Kerstin Reinke (2008): Zur Wirkung phonetischer Mittel in sachlich intendierter Sprechweise bei Deutsch sprechenden Russen. ISBN 978-3-631-57165-1

Bd. 27 Johanna Steinberg (2008): Geflüsterte Plosive. ISBN 978-3-631-57286-3

Bd. 28 Cordula Hunold (2009): Untersuchungen zu segmentalen und supra-segmentalen Ausspracheabweichungen chinesischer Deutschlernender. ISBN 978-3-631-58065-3

Bd. 29 Swetlana Nossok (2009): Kontrastive phonologische und phonetische Analyse Weißrussisch-Deutsch und Analyse interferenzbedingter Ausspracheabweichungen. ISBN 978-3-631-58499-6

Bd. 30 Lutz Christian Anders/Ines Bose (Hg.): Aktuelle Forschungsthemen der Sprechwissenschaft 1. ISBN 978-3-631-59490-2

Bd. 31 Ursula Hirschfeld/Baldur Neuber (Hg.) (2009): Aktuelle Forschungsthemen der Sprechwissenschaft 2. ISBN 978-3-631-59435-3

Bd. 32 Cordula Schwarze (2010): Formen und Funktionen von Topoi im Gespräch. ISBN 978-3-631-58804-8

Bd. 33 Ursula Hirschfeld/Eberhard Stock (Hg.) (2010): Sprechwissenschaftlich-phonetische Untersuchungen zur interkulturellen Kommunikation Russisch-Deutsch. ISBN 978-3-631-60854-8

Bd. 34 Elena Travkina (2010): Sprechwissenschaftliche Untersuchungen zur Wirkung vorgelesener Prosa (Hörbuch). ISBN 978-3-631-60269-0

Bd. 35 Ulrike Sievert/Susanne Voigt-Zimmermann (Hg.) (2011): Klinische Sprechwissenschaft. ISBN 978-3-631-60501-1 


\section{HALLESCHE SCHRIFTEN ZUR SPRECHWISSENSCHAFT UND PHONETIK}

Bd. 36 Hans Krech (2011): Beiträge zur Sprechwissenschaft I. Ausgewählte Schriften zur Therapie von Stimm-, Sprech-, Sprach- und Atmungsstörungen. Herausgegeben von Eva-Maria Krech. Mit einem Beitrag von Lutz Christian Anders. Mit einer Audio-CD. ISBN 978-3-631-61738-0

Bd. 37 Hans Krech (2011): Beiträge zur Sprechwissenschaft II. Die Behandlung gestörter S-Laute. Sprechkundliche Beiträge zur Therapie der Sigmatismen. Herausgegeben von Eva-Maria Krech. Mit einem Beitrag von Volkmar und Renate Clausnitzer. ISBN 978-3-631-61739-7

Bd. 38 Hans Krech (2013): Beiträge zur Sprechwissenschaft III. Ausgewählte Schriften zur Phonetik, Sprechkünstlerischen Gestaltung und Fachgeschichte. Herausgegeben von Eva-Maria Krech. Mit einem Beitrag von Eva-Maria Krech. Mit einer Audio-CD. ISBN 978-3-631-61740-3

Bd. 39 Ines Bose/Baldur Neuber (Hg.) (2011): Interpersonelle Kommunikation: Analyse und Optimierung. ISBN 978-3-631-61276-7

Bd. 40 Gottfried Meinhold/Baldur Neuber (Hg.) (2011): Irmgard Weithase - Grenzgänge. ISBN 978-3-631-61931-5

Bd. 41 Angelika Braun/Christa M. Heilmann (2012): SynchronEmotion. ISBN 978-3-631-61518-8

Bd. 42 Se-Jin Park (2013): Ausspracheabweichungen bei koreanischen Deutschlernenden und Empfehlungen für Ausspracheübungen. ISBN 978-3-631-62637-5

Bd. 43 Lutz Christian Anders/Ines Bose/Ursula Hirschfeld/Baldur Neuber (Hg.) (2013): Aktuelle Forschungsthemen der Sprechwissenschaft 3. ISBN 978-3-631-62883-6

Bd. 44 Ulrike Nespital (2013): Wirkungen des funktionellen Nachvollzugs physiologischer Gesangsstimmen auf die Qualität der Sprechstimme. ISBN 978-3-631-62616-0

Bd. 45 Ulrike Groß/Michael Thiergart (Hg.) (2013): Sprache und Musik. ISBN 978-3-631-64362-4

Bd. 46 Augustin Ulrich Nebert (2013): Der Tonhöhenumfang der deutschen und russischen Sprechstimme. ISBN 978-3-631-64322-8

Bd. 47 Stephanie Kurtenbach/Ines Bose (Hg.) (2013): Gespräche zwischen Erzieherinnen und Kindern. ISBN 978-3-631-62923-9

Bd. 48 Marit Fiedler (2013): Modernhebräisch und Deutsch. ISBN 978-3-631-62686-3

Bd. 49 Salam Omar Mahmood (2014): Ausspracheschwierigkeiten arabischer Deutschlernender aus dem Irak und didaktische Überlegungen zum Ausspracheunterricht. ISBN 978-3-631-65282-4

Bd. 50 Björn Fiedler (2014): Coachingbasiertes Training. ISBN 978-3-631-65434-7

Bd. 51 Ines Bose/Baldur Neuber (Hg.) (2014): Sprechwissenschaft: Bestand, Prognose, Perspektive. ISBN 978-3-631-64740-0 


\section{SCHRIFTEN ZUR SPRECHWISSENSCHAFT UND PHONETIK}

Bd. 1 Anne Zarend (2015): Höflichkeit in der interkulturellen Kommunikation Russisch-Deutsch. Sprechwissenschaftliche Untersuchungen zum Höflichkeitsgrad in telefonischen Servicegesprächen. 324 Seiten. ISBN 978-3-7329-0148-7

Bd. 2 Alexandra Ebel (2015): Aussprache russischer Namen in der bundesdeutschen Standardsprache. Untersuchung zur Akzeptanz verschiedener Eindeutschungsgrade. 252 Seiten. ISBN 978-3-7329-0180-7

Bd. 3 Friderike Lange (2015): Standardaussprache englischer Namen im Deutschen. Sprechwissenschaftliche Untersuchungen zur Eindeutschung. 288 Seiten. ISBN 978-3-7329-0164-7

Bd. 4 Wieland Kranich (2015): Sprechwissenschaftliche Grundlagen der Prosodieperzeption. 412 Seiten. ISBN 978-3-7329-0212-5

Bd. 5 Ursula Hirschfeld/Hans-Joachim Solms/Eberhard Stock (Hg.) (2016): Otto Bremer - Wegbereiter der sprechwissenschaftlichen Phonetik an der Universität Halle. 202 Seiten. ISBN 978-3-7329-0230-9

Bd. 6 Susanne Voigt-Zimmermann/Stephanie Kurtenbach/Gabriele Finkbeiner/ Anke Bergt/Wanda Mainka (Hg.) (2016): Stimmstörungen - ein Fokus der Klinischen Sprechwissenschaft. Aktuelle Beiträge aus Wissenschaft, Forschung und Praxis. 178 Seiten. ISBN 978-3-7329-0240-8

Bd. 7 Ursula Hirschfeld/Friderike Lange/Eberhard Stock (Hg.) (2016): Phonetische und rhetorische Aspekte der interkulturellen Kommunikation. 298 Seiten. ISBN 978-3-7329-0270-5

Bd. 8 Astrid Schmiedel (2017): Phonetik ironischer Sprechweise. Produktion und Perzeption sarkastisch ironischer und freundlich ironischer Äußerungen. 190 Seiten mit CD. ISBN 978-3-7329-0320-7

Bd. 9 Baldur Neuber/Judith Pietschmann (Hg.) (2017): Dialogoptimierung in der Telekommunikation. 222 Seiten. ISBN 978-3-7329-0316-0

Bd. 10 Sandra Reitbrecht (2017): Häsitationsphänomene in der Fremdsprache Deutsch und ihre Bedeutung für die Sprechwirkung. 328 Seiten. ISBN 978-3-7329-0350-4

Bd. 11 Ines Bose/Kati Hannken-Illjes/Ursula Hirschfeld/Baldur Neuber (Hg.) (2017): Forschung und Didaktik der Sprechwissenschaft. Aktuelle Beiträge. 218 Seiten. ISBN 978-3-7329-0358-0

Bd. 12 Judith Pietschmann (2017): Optimierung von Gesprächen in der professionellen Telefonie. 356 Seiten. ISBN 978-3-7329-0395-5 


\section{SCHRIFTEN ZUR SPRECHWISSENSCHAFT UND PHONETIK}

Bd. 13 Heiner Apel (2018): Nachrichten: hörbar informativ. Eine Untersuchung zur Text- und Hörverständlichkeit von Radionachrichten. 466 Seiten. ISBN 978-3-7329-0439-6

Bd. 14 Josefine Méndez (2018): Gesprächseffizienz - Merkmale und Einflussfaktoren. Sprechwissenschaftliche, linguistische und ökonomische Perspektiven. 254 Seiten. ISBN 978-3-7329-0456-3

Bd. 15 André Hüttner (2019): Zur Entwicklung der sprechwissenschaftlichen Phonetik an der Universität Halle (Saale) bis 1961. Mit einem Ausblick auf die weitere Entwicklung des Fachs. 266 Seiten. ISBN 978-3-7329-0537-9

Bd. 16 Ines Bose/Kati Hannken-Illjes/Stephanie Kurtenbach (Hg.) (2019): Kinder im Gespräch - mit Kindern im Gespräch. 300 Seiten. ISBN 978-3-7329-0540-9

Bd. 17 Clara Luise Finke (2019): Senderidentität und Alltagsästhetik. Radiomoderationen in der Primetime. 366 Seiten. ISBN 978-3-7329-0542-3

Bd. 18 Susanne Voigt-Zimmermann/Frank Lorenz/André Hüttner (Hg.) (2019): Sprechwissenschaftliche Phonetik. 364 Seiten. ISBN 978-3-7329-0575-1

Bd. 19 Beata Grzeszczakowska-Pawlikowska (2020): Rhetorische Kompetenzen in der Fremdsprache Deutsch (DaF). Wirkung und Verständlichkeit polnischer Studentinnen in der interkulturellen Hochschulkommunikation. 350 Seiten. ISBN 978-3-7329-0543-0

Bd. 20 Anna Schwenke (2020): Nachrichten: mehr als informativ. Nachrichten-Sprechstile im Radio zwischen Formatierung und Hörverständlichkeit.

424 Seiten. ISBN 978-3-7329-0641-3

Bd. 21 Eberhard Stock (2020): Fachgeschichtliche Notizen: Zur Entwicklung der halleschen Sprechwissenschaft zwischen 1945 und 1990. 178 Seiten. ISBN 978-3-7329-0657-4

Bd. 22 Sarah Heinemann (2020): Die Macht der Gedanken. Eine kritische Analyse der Lehren des Positiven Denkens. 306 Seiten. ISBN 978-3-7329-0660-4

Bd. 23 Robert Skoczek (2020): Aussprache polnischer Namen in der bundesdeutschen Standardsprache. 372 Seiten. ISBN 978-3-7329-0702-1

Bd. 24 Susanne Voigt-Zimmermann (Hg.) (2021): Miteinander sprechen verantwortlich, kompetent, reflektiert. 254 Seiten. ISBN 978-3-7329-0647-5

Bd. 25 Debora Diehl (2021): Aussprache japanischer Namen in der bundesdeutschen Standardsprache. Sprechwissenschaftliche Untersuchungen und Empfehlungen zur Eindeutschung. 318 Seiten. ISBN 978-3-7329-0746-5 

